\title{
Modelagem para dados de sobrevivência com censura dependente: aplicação para análise do tempo de sobrevivência ajustado pela qualidade de vida
}

\author{
Fernando Henrique Sousa Barreto
}

\author{
DisSERTAÇÃO APRESENTADA \\ Instituto de MATEMÁtica e Estatística \\ UNiversidade de SÃo PAulo \\ PARA \\ OBTENÇÃO DO TÍTULO \\ Mestre em Estatística
}

Programa: Estatística

Orientadora: Prof ${ }^{a}$. Dr ${ }^{a}$. Gisela Tunes da Silva

Durante o desenvolvimento deste trabalho o autor recebeu auxílio financeiro da CNPq 
Modelagem para dados de sobrevivência com censura dependente: aplicação para análise do tempo de sobrevivência ajustado pela qualidade de vida

Este exemplar corresponde à redação final da dissertação devidamente corrigida e defendida por Fernando Henrique Sousa Barreto e aprovada pela Comissão Julgadora. 


\section{Modelagem para dados de sobrevivência com censura dependente: aplicação para análise do tempo de sobrevivência ajustado pela qualidade de vida}

Este exemplar corresponde à redação final da dissertação devidamente corrigida e defendida por Fernando Henrique Sousa Barreto e aprovada pela Comissão Julgadora.

Comissão Julgadora:

- Prof ${ }^{a}$. Dr ${ }^{a}$. Gisela Tunes da Silva (Presidente) - IME-USP

- Prof ${ }^{a}$. Dr ${ }^{a}$. Juliana Cobre - ICMC-USP

- Prof. Dr. Antonio Eduardo Gomes - UnB 



\section{Agradecimentos}

Agradeço aos meus pais de coração, Eldo e Flávia, por terem me criado e me incentivado com seu apoio fundamental para ter cursado pós-graduação em São Paulo. Agradeço também ao meu pai, Vicente, por ter sido atencioso e solidário comigo desde muito antes da graduação. Obrigado, pais.

Agradeço ao Prof. João Maurício Araújo Mota, o senhor que me incentivou a trilhar por esse caminho profissional até aqui. O senhor sempre me ajudou muito mais do que sendo um exemplo de professor. Em todos os momentos bons e ruins da graduação até aqui, o senhor esteve presente ajudando de todas as formas possíveis. Obrigado, Prof. Maurício.

Agradeço a Prof ${ }^{a}$. Gisela Tunes da Silva, a senhora que me aceitou como aluno orientando no meu momento mais conturbado em São Paulo. Passei por mais de 2 anos em uma espécie de batalha pessoal incessante em que tive a sorte de tê-la como amiga conselheira. Existem muitos episódios que gostaria de deixar registrado aqui como um gesto de reconhecimento mas não convém expô-los. Entretanto, não posso deixar omitido o fato da sua atenção para com minha pessoa ter sido fundamental. Eu certamente não teria finalizado este trabalho sem a senhora. Obrigado, Prof ${ }^{\text {a }}$. Gisela.

Agradeço a Thainá Soares Silva, ela que se tornou uma grande amiga desde os tempos de graduação na UFC. Só Deus sabe o quanto eu dei - e ainda dou - trabalho. Já passei muito tempo pensando em como agradecer aqui mas sem expor muito as dezenas de centenas de episódios e acredito que não tem modo para agradecer senão pessoalmente. Nenhum texto vai fazer justiça minha eterna dívida contigo. Obrigado, Thainá.

Agradeço o Maxsuel Lima Gomes, ele que virou um grande amigo ainda no curso de Matemática na UFC. Nós dois passamos momentos difíceis e alegres também. Espero que um dia possamos jogar nossa partida de futebol com bolinhas de papel na cesta. Obrigado, Maxsuel.

Agradeço a todos os profissionais que me atenderam prontamente durante minha passagem pelo Programa de Pós-Graduação no IME-USP. Também agradeço ao Conselho Nacional de Desenvolvimento Científico e Tecnológico (CNPq) pelo auxílio financeiro entre março de 2018 a 2020 que ajudaram no desenvolvimento da dissertação.

Muito obrigado! 



\section{Resumo}

BARRETO, F. H. S. Modelagem para dados de sobrevivência com censura dependente: aplicação para análise do tempo de sobrevivência ajustado pela qualidade de vida. 2020. 137 f. Dissertação (Mestrado) - Instituto de Matemática e Estatística, Universidade de São Paulo, São Paulo, 2020.

O Tempo de Sobrevivência Ajustado pela Qualidade de Vida (TSAQV) tem sido bastante utilizado em análise de dados na área médica. A motivação deste trabalho está em um estudo de coorte prospectivo nas UTIs de dois hospitais públicos brasileiros especializados em tratamento oncológico, Instituto do Câncer Dr. Octávio Frias de Oliveira (ICESP) e Fundação Pio XII - Hospital do Câncer de Barretos. Um total de 792 pacientes foram selecionados como participantes do estudo e esses foram acompanhados até óbito ou final do estudo em 24 meses, sendo que o objetivo principal do estudo é avaliar o TSAQV desses pacientes. Entretanto, a dificuldade que surge na análise estatística de dados ao utilizar o TSAQV como desfecho é que a censura passa a ser informativa quando o tempo de sobrevivência é ponderado pela qualidade de vida. Dessa forma, metodologias usuais de análise de sobrevivência são inconsistentes e métodos estatísticos apropriados para a análise do TSAQV precisam ser utilizados. Para tanto, foram estudados e aplicados métodos de estimação com modelos de taxa de falhas proporcionais sob censura dependente usando função de cópula. Foi realizado um extenso estudo de simulação para avaliar a performance dos métodos de estimação em diferentes cenários de simulação. A aplicação dos modelos para dados com presença de censura dependente foi satisfatória, embora persistam algumas dificuldades como o problema de não identificabilidade do parâmetro $\alpha$ da cópula arquimediana. Entretanto, a alternativa usando o índice $C V(\tau)$ mostrou-se uma metodologia confiável para descartar a premissa de censura independente.

Palavras chave: Análise de Sobrevivência; qualidade de vida; censura dependente; cópula. 



\section{Abstract}

\section{BARRETO, F. H. S. Application of copula based models for survival data under dependent}

censoring for the analysis of quality adjusted survival. 2020. $137 \mathrm{f}$. Dissertação (Mestrado) Instituto de Matemática e Estatística, Universidade de São Paulo, São Paulo, 2020.

Recently, Quality-Adjusted Life Year (QALY) has been used as outcome in several health care studies. The motivation of this work was a prospective cohort study at the ICUs of two public Brazilian hospitals, Dr. Octávio Frias de Oliveira Cancer Institute (Instituto ICESP) and Pio XII Foundation - Barretos Cancer Hospital. A total of 792 cancer patients were elected as study participants and followed up until death or end of the study in 24 months. The main objective was to evaluate the QALY of these patients. However, when survival time is weighted by quality of life, censoring becomes informative and hence usual survival analysis methodologies are inconsistent and appropriate statistical methods for the QALY analysis must be used. For this purpose, copula models for survival data with informative censoring were considered. An extensive simulation study was carried out to evaluate the performance of the estimation methods in different simulation scenarios. The application of those models was satisfactory even though some difficulties still persist, such as the problem of non-identifiability of the Archimedean copula $\alpha$ parameter. The alternative, however, using the $C V(\tau)$ index proved to be a reliable methodology to discard the premise of independent censoring.

Keywords: survival analysis; quality of life; dependent censoring; copula. 



\section{Índice}

1 Introdução 1

1.1 Revisão Bibliográfica . . . . . . . . . . . . . . . . . . . 2

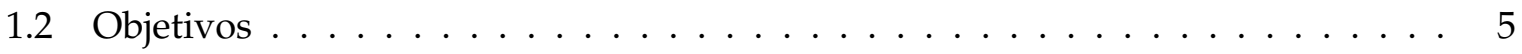

1.3 Organização do Trabalho . . . . . . . . . . . . . . . . . . . . 5

\begin{tabular}{lll}
\hline & Cópulas & 7
\end{tabular}

$2.1 \quad$ Definições e Algumas Propriedades Básicas . . . . . . . . . . . . . . . . . . . . . . 8

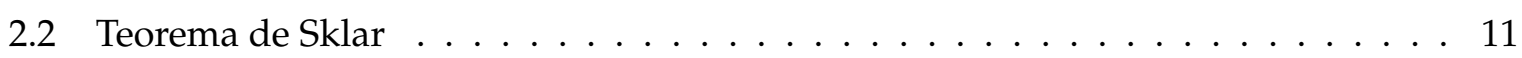

2.3 Classe Arquimediana . . . . . . . . . . . . . . . . . . . . . 13

$2.3 .1 \quad$ Cópula de Clayton $\ldots \ldots \ldots \ldots \ldots$. . . . . . . . . . . . 16

2.3 .2 Cópula de Frank . . . . . . . . . . . . . . . . . . . . . . . . . . . . . . . . . . . . . . . . .

2.3 .3 Cópula de Gumbel . . . . . . . . . . . . . . . . . . . . 17

2.3 .4 Gerador de uma amostra pseudoaleatória . . . . . . . . . . . . . . . . 18

3 Modelos de Taxas de Falha Proporcionais sob Censura Dependente 21

$3.1 \quad$ Estruturas de censura . . . . . . . . . . . . . . . . . . . . . . . . 21

3.2 Problema de Identificabilidade . . . . . . . . . . . . . . . . 24

3.3 Modelo Paramétrico . . . . . . . . . . . . . . . . . . . . . . 25

3.4 Modelo Semiparamétrico I . . . . . . . . . . . . . . . . . . . . 32

$3.4 .1 \quad$ Abordagem Piecewise . . . . . . . . . . . . . . . . . . . 32

3.4 .2 Abordagem M-splines . . . . . . . . . . . . . . . . . . . 34

3.4 .3 Penalidade e Suavização . . . . . . . . . . . . . . . . . . . . . . . 36

3.4 .4 Problema de Otimização com Restrições . . . . . . . . . . . . . . . . . . . . 37

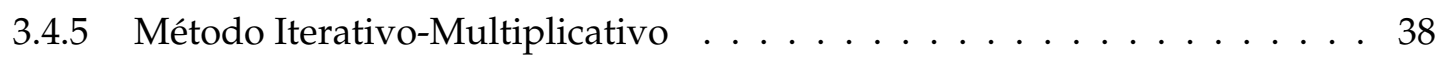

3.4 .6 Propriedades assintóticas $\ldots \ldots \ldots \ldots \ldots$. . . . . . . . . . 41

3.5 Modelo Semiparamétrico II $\ldots \ldots \ldots \ldots$. . . . . . . . . . . . . . . 43

$\begin{array}{llr}4 & \text { Simulações } & 49\end{array}$

4.1 Tempos de Sobrevivência: uma Análise de Sensibilidade . . . . . . . . . . . . . . 49

4.1 .1 Resultados . . . . . . . . . . . . . . . . . . . 56 
4.2 Tempo de Sobrevivência Ajustada pela Qualidade de vida: uma análise de sensibilidade exploratória $\ldots \ldots \ldots \ldots \ldots$. . . . . . . . . . 65

4.2 .1 Resultados . . . . . . . . . . . . . . . . . . . . . . . . 67

5 Aplicação: Análise do Tempo de Sobrevivência Ajustado pela Qualidade de Vida em Pacientes com Câncer $\quad 71$

5.1 Pacientes com Câncer em Estágio Crítico . . . . . . . . . . . . . . . . 71

$5.1 .1 \quad$ Ajuste dos Dados ICESP $\ldots \ldots \ldots \ldots$. . . . . . . . . . . . . 74

\begin{tabular}{lll}
\hline & Considerações Finais & 81
\end{tabular}

\begin{tabular}{|l|l|}
\hline A Principais Resultados para Cópulas Arquimedianas & 83
\end{tabular}

A.1 Cópula de Clayton . . . . . . . . . . . . . . . . . . . 83

A.2 Cópula de Frank. . . . . . . . . . . . . . . . . . . . . . 85

A.3 Cópula de Gumbel-Hougaard . . . . . . . . . . . . . . . . . . . . 88

\begin{tabular}{ll}
\hline B Modelos & 95
\end{tabular}

B.1 Modelo Xu et al. (2018) . . . . . . . . . . . . . . . . . . . . . . . . 95

B.2 Modelo Huang e Zhang (2008) . . . . . . . . . . . . . . . . . . . 106

\begin{tabular}{ll}
\hline C Tabelas & 111
\end{tabular}

\begin{tabular}{ll}
\hline Referências Bibliográficas & 121
\end{tabular} 
canco 1

\section{Introdução}

Segundo Campolina e Ciconelli (2006), "foi particularmente na década de 1960 que o construto qualidade de vida passou a ser entendido como qualidade de vida subjetiva ou qualidade de vida percebida pelas pessoas. Em grande parte influenciado pela Organização Mundial da Saúde (OMS), que declara que a saúde não se restringe à ausência de doença, mas engloba a percepção individual de um completo bem-estar físico, mental e social, o conceito ampliou-se para além da significação do crescimento econômico, buscando envolver os diversos aspectos do desenvolvimento social"(apud Zhan, 1992). O desenvolvimento para sustentar cientificamente um índice que permitiria uma noção sobre qualidade ocorreu após fim da Segunda Guerra Mundial (1939-1945) - como apontou Normilio-Silva (2015) ao escrever "o interesse no conceito de qualidade de vida foi inicialmente compartilhado por cientistas sociais, filósofos e políticos, principalmente nos Estados Unidos da América após a Segunda Guerra Mundial, quando buscavam indicadores capazes de refletir a noção de bem estar social e suprir a falta de humanização com avanço tecnológico da medicina"(apud, Fleck et al., 1999). Ferreira (2002) disse que o interesse na medição de qualidade de vida com relação a saúde cresce desde a década de 1980. Para avaliar uma situação de pacientes diagnosticados com doença degenerativa mas com tratamento paliativo em vigor, pode-se pensar em como medir o estado de saúde - sob certa e bem definida finalidade - para obter uma noção sobre a qualidade de vida daqueles enfermos. Entenda que, para cada indivíduo, pode-se ter uma noção diferente mesmo aqueles que se encontram com mesma condição clínica. No exemplo, o estudo poderia ser elaborado para obter uma avaliação sobre o impacto do tratamento com respeito à perspectiva do paciente em diferentes e predeterminados aspectos da vida que evidenciam os dois componentes citados por Normilio-Silva (2015) quanto à subjetividade (opinião do paciente) e à multidimensionalidade descritas em Group et al. (1995) e Seidl e Zannon (2004).

Segundo Normilio-Silva et al. (2016, tradução do autor), "o Tempo de Sobrevivência Ajustado pela Qualidade de Vida (TSAQV) é uma medida que leva simultaneamente em consideração a duração e a qualidade de vida"(apud, Prieto e Sacristán, 2003) - sendo assim, segundo Ferreira (2002), "permitindo o desenvolvimento de um conceito que combina em uma única medida ganhos na quantidade e na qualidade de vida dos indivíduos". 
Recentemente, o tempo de sobrevivência ajustado pela qualidade de vida tem sido bastante utilizado na análise de dados na área médica. A motivação deste trabalho foi um estudo conduzido no Instituto do Câncer do Estado de São Paulo (ICESP), com resultados preliminares publicados em Normilio-Silva et al. (2016), no qual avaliações periódicas da qualidade de vida, baseadas no questionário EQ-5D-3L, foram obtidas de pacientes com câncer que foram internados na UTI de dois hospitais. Deram entrada nos hospitais um total de 808 indivíduos para avaliação de autorização para entrada em UTI. Daquele anterior total, foram elegíveis 792 pacientes que foram acompanhados até óbito ou final do estudo. O objetivo principal do estudo era avaliar o tempo de sobrevivência ajustado pela qualidade de vida desses pacientes. A dificuldade que surge na análise estatística de dados ao utilizar o TSAQV como desfecho é apontada por Gelber et al. (1989): a censura passa a ser informativa quando o tempo de sobrevivência é ponderado pela qualidade de vida. Dessa forma, metodologias usuais para análise de sobrevivência são inconsistentes e métodos estatísticos apropriados para a análise do TSAQV precisam ser utilizados. Para uma análise descritiva, Zhao e Tsiatis (1997) e Zhao e Tsiatis (1999) propuseram estimadores não paramétricos para a função de sobrevivência baseados em uma técnica conhecida como Inverse Probability of Censoring Weights (IPCW).

Motivados por esses dados, este trabalho de mestrado visa estudar e avaliar modelos de regressão para dados de tempo de sobrevivência com presença de censura dependente que possam ser utilizados para análise dos dados ICESP, ou seja, modelagem de dados TSAQV. Mais especificamente, foram considerados modelos que incorporam a possível dependência entre o tempo de falha $T$ e tempo de censura $C$ por meio de cópulas arquimedianas.

\subsection{Revisão Bibliográfica}

Antes de Zhao e Tsiatis (1997) apresentarem um estimador consistente para funções de TSAQV, artigos como Goldhirsch et al. (1989) e Gelber et al. (1989) tinham trabalhado para estimar a distribuição de sobrevivência. Já em Glasziou et al. (1990) e Gelber et al. (1991) tinham o objetivo de obter estimativa para Tempo de Sobrevivência Médio Ajustado pela Qualidade de Vida (TSMAQV).

Goldhirsch et al. (1989) adentram na discussão sobre a aplicação de quimioterapia alternativa a pacientes que foram diagnosticadas com câncer de mama porque, até então, era muito controverso o que se compreendia por tradeoff entre a toxidade do tratamento e o tempo de sobrevivência livre de doença que, segundo Marotti (2007), é usado mais frequentemente em tratamento alternativo. Para balancear as vantagens entre o tratamento alternativo e a toxidade do mesmo, a Sobrevida Global (OS) foi particionada em tempo de toxidade (TOX), tempo sem sintomas e toxidade (TWiST) e o tempo após a recidiva sistêmica (REL). Eles apresentaram estimativas para as curvas de TSAQV usando o método de Kaplan e Meier (1958) e elas foram comparadas com um teste originalmente proposto por Mantel (1966) que posteriormente foi nomeado por Peto e Peto (1972) como teste de log-rank. Foi proposto um modelo para ajustar as medidas de utilidade relativas ao TOX, TWiST e REL. Entretanto, Gelber et al. (1989) afirmaram que mesmo que o tempo de acompanhamento, o tempo de recidiva e o tempo de duração de 
toxicidade fossem todos independentes, a extração de toxidade induziria uma dependência entre o TWiST e a censura. Portanto, o estimador de Kaplan-Meier é inconsistente e utilizá-lo para estimar curvas de TSAQV seria superestimá-las. Abriu-se, então, a discussão sobre a presença do mecanismo de censura informativa bem como uma técnica para introduzir um limite superior ao tempo de vida para diminuir o viés gerado por aquele mecanismo. Partindo desse fenômeno, Glasziou et al. (1990) propuseram um estimador não enviesado restrito reformulando cada estado para cada histórico individual particionando o tempo. Eles destacam que, muito embora a partição previna o viés, o método possui algumas limitações como por exemplo a falta de abordagens para estudar o efeito das variáveis preditoras. Cerca de dois anos depois, Cox et al. (1992) produziram um texto discutindo sobre uma avaliação global a cerca de Análise de Qualidade de Vida. Eles afirmaram que o principal problema relacionado à medida de qualidade de vida ocorre quando os acompanhamentos são censurados e tais abordagens trazem consigo muito viés agora que o mecanismo de censura é também informativo, mesmo apenas assumindo inocentemente que a variável resposta fosse uma medida $U$ de qualidade de vida em um modelo convencional para Análise de Sobrevivência. Zhao e Tsiatis (1997) dizem que em um típico ensaio clínico, é comum que os pacientes entrem no estudo sobre um período preestabelecido de tempo e o estudo acaba sendo finalizado antes que toda informação sobre aqueles pacientes seja capturada. Muito embora as abordagens em Goldhirsch et al. (1989), Gelber et al. (1989) e Glasziou et al. (1990) tenham limitação, Cox et al. (1992) as qualificaram como atrativas desde que sejam impostas sob certas condições. Foi então que em 1997, Zhao e Tsiatis propuseram um estimador assintoticamente não viciado e normalmente distribuído para a função de TSAQV. Logo em seguida, em Zhao e Tsiatis (1999) foi proposto um estimador mais eficiente mas sem também considerar os possíveis efeitos das variáveis preditoras. Em Zhao e Tsiatis (2001) foi proposto uma abordagem para comparar funções de TSAQV para dois diferentes tratamentos. Em Van Der Laan e Hubbard (1999), foi flexibilizada a suposição de mecanismo com censura independente, agora com a censura podendo depender do tempo do evento de interesse através das variáveis independentes dos pacientes que poderiam estar em função do tempo.

Já foram relatadas diferentes abordagens para problemas de regressão em qualidade de vida como em: 1. Gelber et al. (1993) que utilizaram a abordagem de particionar o modelo de estado de saúde e ajustaram um modelo de taxa de falhas proporcionais para cada período de mudança; 2. Fine e Gelber (2001) que propuseram um modelo de tempo de vida acelerado para a função de sobrevivência e TSAQV; 3. Em Zhao e Tsiatis (2000) foi proposto um estimador para o TSMAQV para dados censurados em que definiram uma classe estimadores que são assintoticamente equivalentes a todos possíveis estimadores assintoticamente normais. Entretanto, não estenderam o modelo com variáveis preditoras; 4. Em Wang e Zhao (2007) foi proposto um modelo para o TSMAQV com variáveis preditoras. Em Wang et al. (2008), foi apresentado como obter o intervalo de confiança para o TSMAQV; 5. Em Tunes-da Silva et al. (2008) foi discutido e proposto um estimador para o TSMAQV baseado em modelos semiparamétricos e paramétricos de multiestado para tempos de permanência em estados de saúde; 6. Shen et al. (2006) propuseram um estimador para a média do tempo de sobrevida ajustado 
pela qualidade de vida de preditores para reabilitação cardíaca; e 7. Rotnitzky et al. (2009) propuseram estimadores para TSMAQV na presença de mecanismos competitivos possivelmente informativos.

Paralelamente, foram propostas algumas abordagens para lidar dados sob esquema de riscos competitivos como em Zheng (1992). Ele discutiu sobre a insuficiência de informação sob o esquema de conhecer apenas os tempos de falha e censura em análise tornando o modelo não identificável para obter as estimativas das curvas de sobrevivência marginais. Também propôs o conhecido estimador Gráfico de Cópula (CG). Em Zheng e Klein (1994) foi proposto um estimador autoconsistente para obter as estimativas das funções de sobrevivência marginais se baseando no fenômeno de riscos competitivos. Em Zheng e Klein (1995) foi discutido novamente sobre a não identificabilidade e sobre uma abordagem que definiria a associação entre os tempos de falha e tempos de censura quanto ao mecanismo de dependência. Eles introduziram a aplicação de funções de cópulas de Sklar (1959) e também demonstraram que se aquela função de cópula é membro da classe arquimediana, então - sob certas condições de regularidade - os dados de riscos competitivos são suficientes para identificar as funções marginais de sobrevivência assim como obter um estimador adequado. Outra vertente da discussão sobre o assunto de identificabilidade foi discutido em Wang (2012) sobre quando duas funções de cópulas arquimedianas diferentes com distintos níveis de dependência podem obter uma mesma estimativa grosseira para as curvas de sobrevivência marginais. Em Wang (2014) foi proposto um novo estimador e em Chandra (2015) foi discutido a identificabilidade, sob certas condições de regularidade, de riscos competitivos como censura dependente induzidos por modelos de fragilidade.

Em Huang e Zhang (2008) foi apresentada uma análise sensitiva para modelo de regressão com censura dependente introduzindo uma verossimilhança parcial estendida usando a ideia sobre "redistribuição de massa" proposta por Efron (1967) e o estimador autoconsistente proposto em Zheng e Klein (1994). Baseando-se no modelo de fragilidade bivariado assumindo cópula descrito em Wienke (2010), Xu et al. (2017) propuseram um modelo de taxa de falhas não-paramétrico para dados com censura informativa à direita. Em Xu et al. (2018) foi proposto um modelo para taxa de falhas proporcionais semi-paramétrico assumindo mecanismo de censura dependente usando cópula e penalizando a função de máxima verossimilhança. Em Xu et al. (2018) foi assumido que, para cada indivíduo, o tempo do evento de interesse e tempo de censura admitem uma estrutura de dependência. Então, eles optaram em aplicar uma função de cópula. No método de estimação para os coeficientes de regressão e coeficientes basais, optou-se pela abordagem de Máxima Verossimilhança Penalizada (MPL) que forneceria uma estimação suavizada, uma vez que a função de penalidade seria usada para obter resultados interpretáveis das funções basais. O método para estimação usando MPL via algoritmo Multiplicativo Iterativo (MI) foi originalmente proposto e bem sucedido na reconstrução de imagem de tomografia em Ma (2010) e para restauração de imagens com restrição de caixas em Ma et al. (2014). Para se ter uma ideia da potencialidade desse algoritmo, segundo Ma et al. (2014, tradução do autor), "problemas de reconstrução de imagem são equivalentes a Modelos Lineares Generalizados (MLG) restringidos de altas dimensões com pelo menos $128^{2}$ 
parâmetros de regressão não-negativos". Entretanto, tem-se o interesse na aplicação que Ma et al. (2014) propuseram para o ajuste de modelos de Cox com censura à direita. $\mathrm{O}$ algoritmo MI se apresentou como uma eficiente abordagem para problemas sob restrição não negativa, requerindo derivadas de até segunda ordem dos coeficientes de regressão e apenas as derivadas de primeira ordem para os coeficientes da aproximação linear às funções basais. Segundo Xu et al. (2017), esse ganho computacional é da ordem de 10 a 20 vezes mais rápido. Todas as propriedades de convergência do algoritmo MI estão disponíveis em Ma et al. (2014).

\subsection{Objetivos}

Essa dissertação teve como objetivo avaliar e aplicar modelos para dados com censura dependente que utilizam função de cópula de membros da classe arquimediana para incorporar a dependência da censura para - mediante a vários cenários em um extenso estudo de simulação - utilizá-los na predição de curvas de sobrevivência na aplicação de dados reais disponibilizados pelo ICESP.

\subsection{Organização do Trabalho}

O Capítulo 2 foi dedicado às noções básicas sobre cópulas com destaque a classe arquimediana, sobretudo, a três dos seus membros, a saber: cópula de Frank, cópula de Clayton e cópula de Gumbel-Hougaard. No decorrer do Capítulo 2, foi escrita uma subseção para introduzir uma geração pseudoaleatória de um par de variáveis aleatórias via cópulas. O Capítulo 3 foi dedicado a apresentação dos modelos de Xu et al. (2018) e Huang e Zhang (2008). Ainda no Capítulo 3, tem-se o desenvolvimento da função de log-verossimilhança - também a função de log-verossimilhança penalizada no caso do modelo de $\mathrm{Xu}$ et al. (2018). Também se tem as aproximações por piecewise, sua extensão e a inclusão de outra abordagem de aproximação chamada M-splines. Em seguida é apresentada a abordagem proposta por Huang e Zhang (2008) bem como o desenvolvimento da função de log-verossimilhança estendida. Prosseguindo, o Capítulo 4 foi dedicado para um estudo de simulações assim como um ingênuo e exploratório estudo simulando dados efetivamente observáveis de TSAQV. O Capítulo 5 foi escrito para apresentar a aplicação do modelo em Xu et al. (2018) ao conjunto de dados ICESP para obter curvas preditoras de sobrevivência. O Capítulo 6 foi escrito para apresentar as considerações finais e propor trabalhos futuros. Por fim, foi desenvolvido um apêndice dividido em três partes A, B e C. A parte A contém os principais cálculos resultantes a respeito de cópula. A parte $\mathrm{B}$ contém os principais cálculos resultantes do Capítulo 3. A parte $\mathrm{C}$ contém todas as tabelas referidas no Capítulo 4. Sobre a ferramenta computacional, utilizamos apenas o software R e finalizamos o trabalho com a versão 3.6.3. Os códigos implementados para este trabalho estarão, quando oportuno, disponíveis livremente pela Prof ${ }^{a}$. Gisela em seu web site https://www.ime.usp.br/ tunes/index.html 


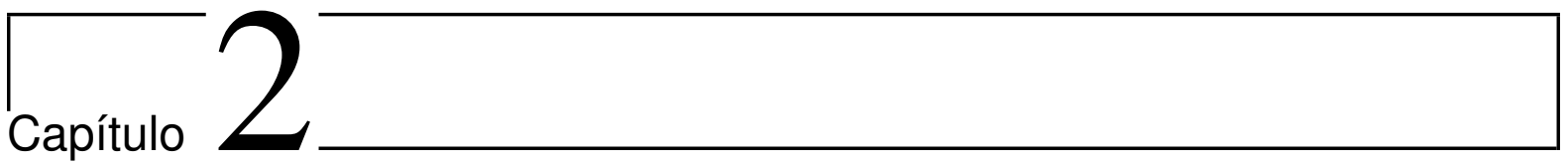

\section{Cópulas}

Esse capítulo é dedicado ao leitor não familiarizado sobre o conceito de Cópula. Todo capítulo tem forte referência ao texto de Nelsen (2006). Dele fora destacado o cerne do presente capítulo, em especial sobre a classe arquimediana. Caso o leitor já tenha conhecimento prévio sobre o assunto, recomendamos seguir a leitura para o próximo capítulo.

Nelsen (2006, tradução do autor) referiu cópulas como "funções que unem ou acoplam funções de distribuição multivariada às suas funções de distribuição marginal unidimensionais" ou, equivalentemente, "funções de distribuição multivariada cujas funções marginais unidimensionais são uniformes no intervalo $(0,1)^{\prime \prime}$. Nelsen (2006) também destaca que a palavra cópula em latim pode ter significado de "ligação", "vínculo" ou "laço". Segundo Emura e Chen (2018, tradução do autor), "grosseiramente, uma cópula é uma função para conectar duas variáveis aleatórias especificando sua estrutura de dependência". Porém, deve-se destacar que esses pontos de vista sobre o estudo de cópulas iniciaram com o trabalho de Sklar (1959) que definiu matematicamente cópulas assim como apresentou um importante resultado que ficou conhecido como Teorema de Sklar. Mais adiante, haverá a definição e explanação do Teorema de Sklar. Todavia, como destacou Nelsen (2006), voltando à linha histórica que parte de Sklar no final da década de 1960, o uso da palavra cópula em título de artigo ou como palavra-chave no índice bibliográfico para Estatística, Current Index Statistics (CIS) somente ocorreu anos depois no trabalho de Schweizer e Wolff (1981). Eles definiram cópula como "uma função que liga uma função de distribuição $n$-dimensional às suas margens unidimensionais e é, em si, uma função de distribuição contínua na unidade $n$-cúbica com margens uniformes" e também demonstraram "que a cópula de um par de variáveis aleatórias $X$ e $Y$ é invariante sob as transformações estritamente crescentes de $X$ e $Y$ como qualquer propriedade da função de distribuição conjunta de $X$ e $Y$ é invariante sob tais transformações é também apenas uma função da sua cópula".

Contudo, Emura e Chen (2018) acrescentaram que o interesse em aplicações de cópulas em Análise de Sobrevivência foi fomentado a partir do trabalho de David George Clayton, introduzindo seu modelo de sobrevivência bivariado em Clayton (1978). Nelsen (2006) comentou que nos primeiros dezoito volumes do CIS (1975-1992) ocorreram onze referências em artigos 
mencionando cópulas. Entre 1993 e 2002, foram mais 71 referências em artigos em dez volumes. $\mathrm{O}$ crescente número de trabalhos publicados fica evidenciado com o fato de que entre 2016 e 2017 foram indexados cinquenta 57 artigos com referência a cópula.

O estudo de cópulas se estende dentro de uma larga classe de aplicações como em processo de controle. Um exemplo de aplicabilidade parte de Emura e Chen (2016) para seleção de genes. Emura e Chen destacaram que, embora pesquisas biomédicas ocorram com o frequente uso do modelo de Cox (1972), tal abordagem necessita da suposição de censura independente um cenário não realístico para muitas aplicações em biomedicina. Levando em consideração o cenário de censura dependente, Emura e Chen ainda apresentaram uma abordagem alternativa à seleção de genes via cópulas. Um outro exemplo pode estar em um estudo sobre síndrome de Lynch. Segundo a Genetics Home Reference (tradução do autor, 2020), "a síndrome de Lynch é uma transmissão autossômica dominante (não polipóide) que consiste em um tipo de câncer hereditário do trato digestivo". Mutações de específicos genes aumentam o risco de desenvolvimento dessa síndrome de tal maneira que essas mutações são riscos competitivos com certa estrutura de dependência. Com essa motivação, Lakhal-Chaieb et al. (2017) analisaram o desenvolvimento de sucessivos cânceres para pessoas com a síndrome de Lynch na presença de riscos competitivos usando cópulas.

O presente capítulo é destinado a uma curta mas essencial apresentação sobre algumas propriedades e resultados a respeito de cópula. Na Seção 2.1. são destacadas as principais definições e algumas propriedades imediatas seguidas de alguns exemplos que foram, em sua maioria, retirados das listas de exercícios de Nelsen (2006). Na Seção 2.2, trata-se de enunciar e discutir o teorema de Sklar. Na sequência, tem-se a Seção 2.3 que apresenta a importante classe arquimediana. Nesta seção, são apresentados os três membros da classe supracitados. Ainda na Seção 2.3. é apresentado a conhecida medida $\tau$ de Kendall que quantifica a estrutura de dependência conhecida como concordância. A última seção foi escrita para apresentar uma abordagem para gerar um par de números aleatórios dependentes via cópulas.

\subsection{Definições e Algumas Propriedades Básicas}

A seguir, são apresentadas algumas notações e denomeações para fixar algumas palavras ou símbolos especiais para o texto adiante. Sendo enfático, a maioria dos resultados e exemplos a seguir foram retirados do livro de Nelsen (2006).

Primeiramente, considere duas variáveis aleatórias $T$ e $C$ com funções de distribuição conjunta e marginais definidas como em Roussas (1997) e aqui denotadas por $F_{T, C}(t, c), F_{T}(t)$ e $F_{C}(c)$, respectivamente. Assim, denotam-se as funções de sobrevivência conjunta e marginais como $S_{T}(t)=1-F_{T}(t)$ e $S_{C}(c)=1-F_{C}(c)$.

Aqui é denotado o conjunto dos números reais por $\mathbb{R}$, com $\mathbb{R}^{2}$ denotando o plano real $\mathbb{R} \times \mathbb{R}$. Como o intervalo $[0,1]$ será extensivamente usado durante o texto, aqui ele é escrito como $I$ e o quadrado unitário $I \times I$, por vezes, como $I^{2}$. Um retângulo no $\mathbb{R}^{2}$ é o produto cartesiano, $B$, entre dois intervalos fechados $\left[t_{1}, c_{1}\right] \times\left[t_{2}, c_{2}\right], \operatorname{com}\left(t_{1}, c_{1}\right),\left(t_{1}, c_{2}\right),\left(t_{2}, c_{1}\right)$ e $\left(t_{2}, c_{2}\right)$ vértices do retângulo. 
A seguir, será dado mais destaque para algumas definições antes de escrever formalmente uma cópula.

Definição 1 Uma função $S$ é uma função real do tipo $2 P$ cujo domínio, $\operatorname{dom} S$, é um subconjunto de $\mathbb{R}^{2}$ e contradominio, ran $S$, é um subconjunto de $\mathbb{R}$.

Exemplo 1 Seja uma função $S(x, y)=x^{2}+y-1$. Com dominio dom $S=I^{2}$ e contradomínio $\operatorname{ran} S=[-1,1], \log o S$ é uma função real do tipo $2 P$.

Exemplo 2 Sejam $T$ e $C$ variáveis aleatórias contínuas. A função de sobrevivência, $S_{T, U}$,é uma função real do tipo $2 P$.

Definição 2 Sejam $M_{1}$ e $M_{2}$ subconjuntos não vazios de $\mathbb{R}$ e seja $S$ uma função real $2 P$ tal que seu dominio seja dom $S=M_{1} \times M_{2}$. Agora seja $B=\left[t_{1}, c_{1}\right] \times\left[t_{2}, c_{2}\right]$ um retângulo de vértices pertencentes ao dom $S$. Então o volume-S de B é dado por

$$
V_{S}(B)=S\left(t_{2}, c_{2}\right)-S\left(t_{2}, c_{1}\right)-S\left(t_{1}, c_{2}\right)+S\left(t_{1}, c_{1}\right)
$$

Exemplo 3 Seja $S(t, c):(t, c) \mapsto \min (t, c)$, com $\operatorname{dom} S=[0,5]^{2}$ e o quadrado $B=I^{2}$. Pela equação definição 2, o volume-S de $I^{2} e ́ V_{S}\left(I^{2}\right)=1$. Para $B=\operatorname{dom} S$, temos $V_{S}(B)=5$. Para $a, b \in[0,5]$ tal que $a<b$ e $B=[a, b]^{2}$, temos $V_{S}(B)=b-a>0$.

Exemplo 4 Seja $S(t, c):(t, c) \mapsto \max (t, c)$, com $\operatorname{dom} S=[0,5]^{2}$ e o quadrado $B=I^{2}$. Pela equação 2.1), o volume-S de $I^{2} e ́ V_{S}\left(I^{2}\right)=-1$. Para $B=\operatorname{dom} S$, temos $V_{S}(B)=-5$. Para $a, b \in[0,5]$ tal que $a<b$ e $B=[a, b]^{2}$, temos $V_{S}(B)=a-b<0$.

Definição 3 Seja S uma função real do tipo $2 P$. Dizemos que S é uma função duplamente crescente se $V_{S}(B) \geq 0$ para todo retângulo B cujos vértices pertencem a $\operatorname{dom} S$.

Exemplo 5 Seja a função $S$ definida no Exemplo 3. Para $a, b \in[0,5]$ tal que $a<b e B=[a, b]^{2}$, temos $V_{S}(B)=b-a>0$. Então $S$ é função duplamente crescente.

Exemplo 6 Seja a função $S$ definida no Exemplo 4. Para $a, b \in[0,5]$ tal que $a<b$ e $B=[a, b]^{2}$, temos $V_{S}(B)=a-b<0$. Então $S$ não é função duplamente crescente.

Exemplo 7 (Família Caudras-Augé). Seja $\alpha \in$ I e faça

$$
K_{\alpha}(a, b)=[\min (a, b)]^{\alpha}[a b]^{1-\alpha}=\left\{\begin{array}{ll}
a b^{1-\alpha} & a \leq b \\
a^{1-\alpha} b & a \geq b
\end{array}, \quad(a, b) \in I^{2} .\right.
$$

Adiante, é apresentado que essa família é uma cópula e, portanto, função duplamente crescente. Para mais informações da construção dessa família e o estudo sobre os aspectos da estrutura de dependência, veja Cuadras e Augé (1981) e Bolbolian et al. (2010). 
Definição 4 (Cópula). Define-se uma subcópula como uma certa classe de funções de solo e duplamente crescentes com marginais. Então se define uma cópula como uma subcópula com domínio no quadrado unitário $I^{2}$.

Para esse texto, tem-se interesse em cópulas bidimensionais. Portanto, daqui em diante todos os resultados são formulados para o caso bidimensional.

Definição 5 (Subcópula). Uma cópula (subcópula bidimensional), denotada por $K(\cdot, \cdot)$ ) é uma função com as seguintes propriedades:

1. o domínio $\operatorname{dom} K=M_{1} \times M_{2}$, com $M_{1}$ e $M_{2}$ subconjuntos de I contendo 0 e 1 ; $e$

2. Ké uma função solo e duplamente crescente, i.e., para todo $t \in M_{1}$ e $u \in M_{2}$ tal que

2.1 (função solo)

$$
K(t, 0)=0=K(0, c)
$$

$e$

$$
K(t, 1)=t \quad e \quad K(1, c)=c
$$

2.2 (duplamente crescente) para todo $t_{1}, c_{1} \in M_{1}$ e $t_{2}, c_{2} \in M_{2}$ com $t_{1} \leq t_{2}$ e $c_{1} \leq c_{2}$, tem-se

$$
K\left(t_{2}, c_{2}\right)-K\left(t_{2}, c_{1}\right)-K\left(t_{1}, c_{2}\right)+K\left(t_{1}, c_{1}\right) \geq 0
$$

Pela desigualdade (2.5), pode-se escrever uma cópula como um volume-S do retângulo $B=[u, 0] \times[0, c], \operatorname{com}(c, c),(c, 0),(0, c)$ e $(0,0)$ vértices que pertencem a $M_{1} \times M_{2}$.

Exemplo 8 Sejam as funções: (a) $M(t, c)=\min (t, c) ;(b) W(t, c)=\max (t, c) ; e(c) \Pi(t, c)=c t$. Para demonstrar que essas funções são cópulas, acompanhe:

1. todas as funções são confortáveis quanto a suposição do dominio ser um subconjunto de $I^{2} ; e$

2. função solo e duplamente crescente

2.1 (função solo)

$$
\begin{array}{ll}
W(t, 0)=\max (t-1,0)=0 & M(t, 0)=\min (t, 0)=0 \\
W(0, c)=\max (c-1,0)=0 & M(0, c)=\min (0, c)=0 \\
W(t, 1)=\max (t+0,0)=t & M(t, 1)=\min (t, 1)=t \\
W(1, c)=\max (c+0,0)=u & M(1, c)=\min (1, c)=u \\
\Pi(t, 0)=t \cdot 0=0 & \\
\Pi(0, c)=0 \cdot c=0 & \\
\Pi(t, 1)=t \cdot 1=t & \\
\Pi(1, c)=1 \cdot c=c . &
\end{array}
$$


2.2 (duplamente crescente). Seja $B=\left[a_{1}, b_{1}\right] \times\left[a_{2}, b_{2}\right]$. Então

$$
\begin{aligned}
& V_{M}(B)=\left\{\begin{array}{lc}
a_{2}-b_{1}, & \text { se } b_{1} \leq a_{2} \\
0, & \text { caso contrário }
\end{array}, \quad V_{W}(B)=\left\{\begin{array}{cc}
b_{1}-a_{2}, & \text { se } a_{2} \leq b_{1} \\
0, & \text { caso contrário }
\end{array},\right. \text { e }\right. \\
& V_{\Pi}(B)=\left(a_{2}-a_{1}\right)\left(b_{2}-b_{1}\right) \geq 0 .
\end{aligned}
$$

Exemplo 9 Para provar que a família Caudras-Augé (2.2) é uma cópula, acompanhe:

1. o domínio dom $K_{\alpha}=I^{2}$ satisfaz a primeira propriedade ; $e$

2. função solo e duplamente crescente

2.1 (função solo)

$$
\begin{array}{llll}
K_{\alpha}(a, 0)=a \cdot 0^{1-\alpha}=0 & \text { e } & K_{\alpha}(0, b)=a^{1-\alpha} \cdot 0=0, & a \leq b, \\
K_{\alpha}(a, 1)=a \cdot 1^{1-\alpha}=a & e & K_{\alpha}(1, b)=1^{1-\alpha} \cdot b=b, & a \geq b .
\end{array}
$$

2.2 (duplamente crescente) $B=\left[a_{1}, b_{1}\right] \times\left[a_{2}, b_{2}\right]$,

2.2.1 caso I: $a_{1} \leq b_{1} e a_{2} \leq b_{2}$.

$$
\begin{aligned}
V_{K_{\alpha}}(B) & =K_{\alpha}\left(a_{2}, b_{2}\right)-K_{\alpha}\left(a_{2}, b_{1}\right)-K_{\alpha}\left(a_{1}, b_{2}\right)+K_{\alpha}\left(a_{1}, b_{1}\right) \\
& =a_{2} b_{2}^{1-\alpha}-a_{2} b_{1}^{1-\alpha}-a_{1} b_{2}^{1-\alpha}+a_{1} b_{1}^{1-\alpha} \\
& =a_{2}\left(b_{2}^{1-\alpha}-b_{1}^{1-\alpha}\right)-a_{1}\left(b_{2}^{1-\alpha}-b_{1}^{1-\alpha}\right) \\
& =\left(a_{2}-a_{1}\right)\left(b_{2}^{1-\alpha}-b_{1}^{1-\alpha}\right) \geq 0 .
\end{aligned}
$$

2.2.1 caso II: $a_{1} \geq b_{1} e a_{2} \geq b_{2}$.

$$
\begin{aligned}
V_{K_{\alpha}}(B) & =K_{\alpha}\left(a_{2}, b_{2}\right)-K_{\alpha}\left(a_{2}, b_{1}\right)-K_{\alpha}\left(a_{1}, b_{2}\right)+K_{\alpha}\left(a_{1}, b_{1}\right) \\
& =a_{2}^{1-\alpha} b_{2}-a_{2}^{1-\alpha} b_{1}-a_{1}^{1-\alpha} b_{2}+a_{1}^{1-\alpha} b_{1} \\
& =a_{2}^{1-\alpha}\left(b_{2}-b_{1}\right)-a_{1}^{1-\alpha}\left(b_{2}-b_{1}\right) \\
& =\left(a_{2}^{1-\alpha}-a_{1}^{1-\alpha}\right)\left(b_{2}-b_{1}\right) \geq 0 .
\end{aligned}
$$

\subsection{Teorema de Sklar}

Sklar introduziu cópula em seu estudo sobre espaço métrico probabilístico em 1959 e seu teorema é a base de muitas aplicações. É também por esse teorema que pode-se explicar o relacionamento entre distribuições multivariadas com suas respectivas distribuições marginais.

Teorema 1 (Teorema de Sklar). Seja $S_{T, C}(\cdot, \cdot)$ função de sobrevivência conjunta das variáveis aleatórias $T$ e $C$, com as respectivas funções de sobrevivência marginais $S_{F}(\cdot)$ e $S_{C}(\cdot)$. Então existe uma cópula, 
$K(\cdot, \cdot)$, tal que para todo $t, c \in \mathbb{R}$, pode-se escrever

$$
S_{T, C}(t, c)=K\left(S_{T}(t), S_{C}(c)\right), \quad(t, c) \in A_{T} \times A_{C},
$$

com $A_{T}$ e $A_{C}$ suportes das distribuições de $T$ e $C$, respectivamente. Caso $T$ e $C$ sejam contínuas, então a cópula $K(\cdot, \cdot)$ é única. Caso contrário, a cópula $K(\cdot, \cdot)$ é unicamente determinada em $\operatorname{ran} S_{T} \times \operatorname{ran} S_{C}$. Reciprocamente com $K(\cdot, \cdot)$ uma cópula e $S_{T}(\cdot)$ e $S_{C}(\cdot)$ funções de sobrevivência, então $S_{T, C}(\cdot, \cdot)$ é função de sobrevivência conjunta com marginais $S_{T}(\cdot)$ e $S_{C}(\cdot)$.

Consequências imediatas a respeito do teorema a cima são:

- as cópulas permitem modelar distribuições marginais e a estrutura de dependência das variáveis aleatórias separadamente; e

- pelas relações $S_{T}(t)=x$ e $S_{C}(c)=y$, pode-se obter as inversas $t=S_{T}^{-1}(x)$ e $c=S_{C}^{-1}(y)$ e substituindo $t$ e $c$ em $S_{T, U}(t, u)=K\left(S_{T}(t), S_{C}(c)\right)$, resulta em

$$
K(x, y)=S_{T, C}\left(S_{T}^{-1}(x), S_{C}^{-1}(y)\right), \quad(x, y) \in I^{2} .
$$

Outra consequência menos evidente do teorema de Sklar é que a abordagem a cima permite a construção de cópulas apenas "invertendo", dado uma função de distribuição conjunta $S_{T, C}(\cdot, \cdot)$, as funções de distribuições marginais. Embora tenha outras abordagens para construir uma cópula (veja Nelsen, 2006), este texto focou apenas nesta abordagem discutida.

Exemplo 10 Considere T e C variáveis aleatórias com função de sobrevivência conjunta

$$
S_{T, C}(t, c)=1-\left(1+\mathrm{e}^{-\mathrm{t}}+\mathrm{e}^{-\mathrm{c}}\right)^{-1},(\mathrm{t}, \mathrm{c}) \in \mathbb{R} \text {. }
$$

Sabe-se que a função densidade de probabilidade é

$$
f_{T, C}(t, c)=-\frac{\partial^{2}}{\partial c \partial t} S_{T, C}(t, c)=\frac{2 \mathrm{e}^{-\mathrm{t}-\mathrm{c}}}{\left(1+\mathrm{e}^{-\mathrm{t}}+\mathrm{e}^{-\mathrm{c}}\right)^{3}} .
$$

Agora para obter as distribuições marginais $F_{T}(t)=\int_{-\infty}^{\infty} f_{T}(t, c) d c$ e $F_{C}(c)=\int_{-\infty}^{\infty} f_{T, C}(t, c) d t$, usa-se, por exemplo, a substituição $u=1+\mathrm{e}^{-\mathrm{t}}+\mathrm{e}^{-\mathrm{c}}$ para concluir que

$$
S_{T}(t)=1-\frac{1}{1+\mathrm{e}^{-\mathrm{t}}} \quad e \quad S_{C}(c)=1-\frac{1}{1+\mathrm{e}^{-\mathrm{c}}} .
$$

Seguidamente, as inversas marginais são

$$
S_{T}^{-1}(t)=-\log \left(\frac{t}{1-t}\right) \quad \text { e } \quad S_{C}^{-1}(c)=-\log \left(\frac{c}{1-c}\right)
$$

Substituindo em $S_{T, C}\left(S_{T}^{-1}(t), S_{C}^{-1}(c)\right)$, então o teorema de Sklar garante que

$$
K(t, c)=\frac{t c}{t+c-t c}
$$


No Exemplo 7 foi apresentado a família de Caudras-Augé que também é uma cópula. Agora que foi escrita a definição de uma cópula e foi enunciado o teorema de Sklar, vale destacar a notação para distinguir quando, por exemplo, uma cópula é escrita $K_{\alpha}(\cdot, \cdot)$ ou apenas por $K(\cdot, \cdot)$ : quando uma cópula é indexada por $\alpha$, então fica definido que ela pertence a uma família de um parâmetro. $\mathrm{O}$ mesmo para uma cópula escrita por $K_{\alpha_{1}, \alpha_{2}}(\cdot, \cdot)$, isto é, a cópula é membro de uma família de dois parâmetros. Entretanto, este trabalho focou apenas em três famílias de um parâmetro que serão apresentadas na próxima seção.

Essa subseção é concluída com um teorema a respeito de derivadas parciais de cópulas. Esses resultados são importantes para a compreensão de como gerar uma amostra pseudoaleatória usando uma cópula como abordagem.

Teorema 2 Seja $K(t, c)$ uma cópula. Para qualquer $y \in I$, a derivada parcial de primeira ordem com relação a t existe para quase todo t tal que, para dado te $c$, tem-se

$$
0 \leq \frac{\partial}{\partial t} K(t, c) \leq 1 .
$$

Analogamente, para qualquer $t \in I$, a derivada parcial de primeira ordem com relação a c, existe para quase todo $c$, tal que para dado te c, tem-se

$$
0 \leq \frac{\partial}{\partial c} K(t, c) \leq 1 .
$$

Prova. Veja Nelsen (2006), páginas 13-14.

\subsection{Classe Arquimediana}

Dentre as classes de cópulas, aqui é destacada a classe arquimediana com alguns dos seus principais resultados que serão utilizados mais adiante para a construção da estrutura de dependência nos modelos com censura dependente.

Primeiramente, o nome para essa classe foi adotado porque esse específico conjunto de famílias possui uma propriedade que satisfaz um axioma arquimediano (veja Nelsen, 2006, páginas 115-123). Agora, umas das atrações dessa classe é o fato de que seus membros são escritos por expressões analíticas e também por algumas de suas propriedades serem obtidas sem apelo a uma complexidade matemática - tão importante especialmente para a obtenção da fórmula analítica da cópula como, consequentemente, as funções inversas das respectivas componentes. A classe arquimediana possui muitos membros, mas aqui serão destacados apenas três. Na parte A do Apêndice, são apresentados vários resultados de derivadas para cada um dos três membros. Esses resultados serão importantes para os próximos capítulos, começando pela definição da função pseudo-inversa.

Antes de apresentar os membros em destaque e seus resultados, serão expostos algumas definições e resultados importantes à classe arquimediana e também uma breve discussão sobre medidas de dependência. 
Definição 6 Considere uma função $\varphi: I \longrightarrow[0, \infty)$ de tal sorte que $\varphi(t)$ seja função contínua $e$ estritamente decrescente $\operatorname{com} \varphi(1)=0$. Agora, seja $\varphi^{[-1]}(t)$ a pseudo-inversa de $\varphi(t)$ escrita como

$$
\varphi^{[-1]}(t)=\left\{\begin{array}{lc}
\varphi^{-1}(t), & 0 \leq t \leq \varphi(t) \\
0, & \varphi(0) \leq t<\infty
\end{array}\right.
$$

A pseudo-inversa é uma função contínua, não crescente em $[0, \infty)$ e estritamente decrescente em $[0, \varphi(0)]$. Já a função $\varphi(t)$ da definição 6 é comumente chamada de gerador de uma cópula. Note que $\varphi^{[-1]}(\varphi(t))=t$ quando $t \in I$ e $\varphi\left(\varphi^{[-1]}(t)\right)=\min (t, \varphi(0))$. Uma última consequência imediata entre o gerador e sua pseudo-inversa é se $\varphi(0)=\infty$, então $\varphi^{[-1]}(t)=\varphi^{-1}(t)$.

Teorema 3 Sejam $\varphi(t)$ e $\varphi^{[-1]}(t)$ as funções escritas na Definiçãa 6. Então considere uma função $K: I^{2} \longrightarrow$ I de tal sorte escrita como

$$
K(t, c)=\varphi^{[-1]}(\varphi(t)+\varphi(c))
$$

Então $K(t, c)$ é cópula se, e somente se, a função $\varphi(t)$ é convexa.

Prova. Veja Nelsen (2006), páginas 110-111.

Observe que $\varphi(t)$ é convexa se, e somente se, sua pseudo-inversa também é convexa. De imediato, a cópula $K(t, c)$ também é função convexa. Embora o destaque seja para cópulas bidimensionais, vale ressaltar que, em um estudo multidimensional, o gerador e sua pseudoinversa podem reduzir um problema $n$ dimensional para unidimensional. Existem várias propriedades para uma cópula arquimediana. Aqui destacam-se três destas:

- $K(t, c)$ é simétrica com relação a $t$ e $c$, isto é, $K(t, c)=K(c, t), \forall t, c \in I$;

- $K(t, c)$ é associativa, isto é, $K(K(t, c), w)=K(t, K(c, w)), \forall t, c$ e $w \in I$; e

- para qualquer constante $w>0, \varphi^{*}(t)=w \varphi(t)$ também é gerador de $K(t, c)$.

\section{Medidas de Dependência}

Em quase todos textos que foram referenciados sobre cópulas, geralmente há um capítulo ou pelo menos uma seção à parte que trata de medidas de dependência entre variáveis aleatórias. Desde a introdução se destaca o conceito de estrutura de dependência sendo que são por essas medidas de dependência que pode-se compreendê-la. Nelsen (2006) destacou quatro medidas, a saber: o coeficiente de correlação linear de Pearson, a medida $\tau$ de Kendall, a medida $\rho$ de Spearman e a medida de Gini. Aqui não é aberta uma discussão aprofundada sobre todas medidas citadas, mas pretende-se destacar alguns pontos importantes e explicar o motivo de escolher a medida $\tau$ de Kendall para ser usada mais adiante nos modelos do Capítulo 3 . $\mathrm{O}$ primeiro ponto positivo a respeito daquelas medidas é o fato de que elas somente dependem da cópula que corresponde as variáveis aleatórias.

O coeficiente de correlação linear de Pearson tem alguns fatos não atrativos como, por exemplo, apesar da correlação ter a propriedade de ser invariante a respeito de uma transformação 
linear, o mesmo não é verdade para quando se tem uma transformação não linear em $T$ e $C$. Talvez o mais danoso fato para o coeficiente de Pearson é que seu coeficiente não é uma medida de concordância conforme a Definição 7.

Definição 7 Uma medida de associação, $\kappa$, entre duas variáveis aleatórias contínuas, $T$ e $C$, com cópula $K(t, c)$ é dita medida de concordância se ela satisfaz as seguintes propriedades:

1. $\kappa$ é definida para todo par $T, C$ de variáveis aleatórias contínuas;

2. $\kappa$ é simétrica com relação as variáveis aleatórias, isto é, $\kappa_{T, C}=\kappa_{C, T}$;

3. se T e C são independentes, então $\kappa_{T, C}=0$;

4. $-1 \leq \kappa_{T, C} \leq 1, \kappa_{T, T}=1$ e $\kappa_{T,-T}=-1$;

5. $\kappa_{-T, C}=\kappa_{T,-C}=-\kappa_{T, C} ;$

6. se $K_{1}$ e $K_{2}$ são cópulas tais que $K_{1} \prec K_{2}$, então $\kappa_{K_{1}} \leq \kappa_{K_{2}}$;

7. se $\left\{\left(T_{n}, C_{n}\right)\right\}$ é uma sequência de variáveis aleatórias contínuas com cópulas $K_{n}$ e, com $\left\{K_{n}\right\}$ convergindo pontualmente para $K_{n}$, então $\lim _{n \rightarrow \infty} \kappa_{K_{n}}=\kappa_{K}$.

Entretanto, as medidas $\tau$ de Kendall e $\rho$ de Spearman satisfazem a definição]7(veja Nelsen, 2006, página 169). Sobre a medida de Spearman, pode-se ter uma interpretação geométrica de $\rho_{T, C}$, pois, ela é proporcional ao volume entre as áreas da cópula $K(t, c)$ e a cópula $\Pi(t, c)=t c$. A expressão da medida de Spearman é escrita como

$$
\rho_{T, C}=12 \int_{0}^{1} \int_{0}^{1}[K(t, c)-t c] d t d c .
$$

O resultado a cima pode dificultar uma forma analítica para o coeficiente. Então esse é um fato menos atrativo no coeficiente $\rho$ de Spearman. Por outro lado, o coeficiente $\tau$ de Kendall pode ser escrito como

$$
\tau_{T, C}=1+4 \int_{0}^{1} \frac{\varphi(t)}{\varphi^{\prime}(t)} d t
$$

A expressão a cima pode facilitar uma forma analítica para o coeficiente. É justamente o que acontece com 2 dos 3 membros escolhidos neste texto em que, excetuando a cópula de Frank, possuem coeficientes de forma analítica simples. Há também uma relação entre os dois últimos coeficientes (veja Nelsen, 2006, página 175). Daqui em diante, são omitidos os índices a respeito das variáveis aleatórias nos coeficientes - salvo necessidade para evitar ambiguidade.

Os coeficientes de Kendall e Spearman são úteis para variáveis aleatórias ordinais. Quando se está trabalhando com variáveis aleatórias nominais, obviamente $\tau$ e $\rho$ não são apropriados para sua medida de dependência. Então para esse tipo de situação, a medida de Gini é indicada (veja Nelsen, 2006, página 180). Como esse texto não trabalhou com variáveis do tipo nominal, então a medida de Gini aqui não foi enfatizada. 
Toda essa discussão um tanto superficial, dado o vasto assunto, sobre medidas de dependência foi elaborado para introduzir a importante relação entre o parâmetro da família que define a cópula e os coeficientes. É por intermédio desta relação que pode-se estudar o grau de dependência entre as funções basais no modelo.

\subsubsection{Cópula de Clayton}

A cópula de Clayton ou, por vezes, cópula de Pareto é uma família de cópulas que foi discutida primeiramente por Clayton (1978). Emura e Chen (2018) apresentaram uma linha do tempo narrando as principais contribuições para este membro arquimediano. Na tabela $4.1 \mathrm{em}$ Nelsen (2006), a cópula de Clayton é escrita como

$$
\begin{aligned}
K_{\alpha}(t, c) & =\left[\max \left\{t^{-\alpha}+c^{-\alpha}-1,0\right\}\right]^{-\frac{1}{\alpha}}, \\
\varphi(t) & =\frac{1}{\alpha}\left(t^{-\alpha}-1\right), \\
\tau & =\frac{\alpha}{\alpha+2},
\end{aligned}
$$

para $\alpha \in[-1, \infty)-\{0\}$ e $\tau$ sendo o coeficiente de Kendall. O gerador da cópula de Clayton é não estrito uma vez que $\varphi(0)=-\frac{1}{\alpha}$ e, então, uma inconveniência matemática ocorre para o domínio da pseudo-inversa que será dom $\varphi^{-1}=\left[0,-\frac{1}{\alpha}\right]$. Portanto, utilizando a Definição 6 , escreve-se a pseudo-inversa como $\varphi^{-1}(t)=\{\max (0, \alpha t+1)\}^{\frac{1}{\alpha}}$, resultando em uma expressão mais confortável

$$
K_{\alpha}(t, c)=\left\{\begin{array}{cc}
\left(t^{-\alpha}+c^{-\alpha}-1\right)^{-\frac{1}{\alpha}}, & \alpha>0, \\
t c, & \alpha=0, \\
{\left[\max \left\{t^{-\alpha}+c^{-\alpha}-1,0\right\}\right]^{-\frac{1}{\alpha}}} & -1 \leq \alpha<0 .
\end{array}\right.
$$

A expressão a cima permite que o coeficiente $\tau$ de Kendall agora, matematicamente, tenha valor zero. Ainda sobre o gerador da cópula de Clayton, ele também é um gerador estrito, isto é, sua função pseudo-inversa é escrita como $\varphi^{-1}:[0, \infty] \longrightarrow[0,1]$ (veja Nelsen, 2006, páginas 111-113). Sobre o espaço paramétrico de $\alpha, \alpha \in(-1,0)$ é útil quando existe uma associação negativa. Perceba que

- para $\alpha>0$, então existe uma associação (concordância) positiva $(\tau>0)$;

- para $\alpha \longrightarrow 0$, então existe independência $(\tau=0)$; e

- para $\alpha<0$, então existe uma associação (concordância) negativa $(\tau<0)$.

No Apêndice A estão disponíveis vários resultados de derivadas de até terceira ordem da função cópula de Clayton porque são demonstrações de expressões analíticas extensas que foram necessárias obter para construir rotinas no software $\mathrm{R}$. 


\subsubsection{Cópula de Frank}

Segundo Nelsen (2006), a cópula de Frank (1979) foi introduzida em um contexto fora da área de Estatística. A propriedade única dentre os demais membros da classe arquimediana é a indiferença ao transladar a origem $(0,0)$ da cópula. Por transladar, admita uma cópula $K_{\alpha}(1-t, 1-c)$ que assim translada a origem para o ponto $(1,1)$. Nelsen $(2006)$ denominou $K_{\alpha}(1-t, 1-c)$ como cópula de sobrevivêncid 1 . Em Mari e Kotz $(2001)$, a cópula $K_{\alpha}(1-t, 1-c)$ é denominada por cópula complementar. A seguir, a expressão da cópula de Frank

$$
\begin{aligned}
K_{\alpha}(u, v) & =-\frac{1}{\alpha} \log \left(1+\frac{\left(e^{-\alpha u}-1\right)\left(e^{-\alpha v}-1\right)}{e^{-\alpha}-1}\right), \\
\phi(t) & =-\log \left(\frac{e^{-\alpha t}-1}{e^{-\alpha}-1}\right), \\
\tau & =1+\frac{4}{\alpha}\left[D_{1}(\alpha)-1\right],
\end{aligned}
$$

com $D_{1}(\alpha)=\frac{1}{\alpha} \int_{0}^{\alpha} \frac{t}{e^{t}-1} d t$. A função inversa para a cópula de Frank é

$$
\phi^{-1}(t)=-\frac{1}{\alpha} \log \left(1+e^{-t}\left(e^{-\alpha}-1\right)\right)
$$

- para $\alpha>0$, então existe uma associação (concordância) positiva $(\tau>0)$;

- para $\alpha \longrightarrow 0$, então existe independência $(\tau=0)$; e

- para $\alpha<0$, então existe uma associação (concordância) negativa $(\tau<0)$.

Agora deve-se destacar que Xu et al. (2018) preferiram reparametrizar $\alpha \operatorname{comos}=-\alpha$ o que resultaria em

$$
\begin{aligned}
K_{s}^{*}(u, v) & =\frac{1}{s} \log \left(1+\frac{\left(e^{s u}-1\right)\left(e^{s v}-1\right)}{e^{s}-1}\right) \\
\phi^{*}(t) & =\log \left(\frac{e^{s t}-1}{e^{s}-1}\right) \\
\tau^{*} & =1-\frac{4}{s}\left[D_{1}(-s)-1\right] .
\end{aligned}
$$

Enfatiza-se que reparametrização é apenas uma questão de preferência e, então, o texto continua de acordo com a parametrização da tabela 4.1 Nelsen (2006). Assim como a cópula de Clayton, estão disponíveis no Apêndice A vários resultados com suas respectivas demonstrações para a cópula de Frank.

\subsubsection{Cópula de Gumbel}

Nelsen (2006) mencionou essa família membro como cópula de Gumbel assim como muitos autores como cópula de Gumbel (1960), mas ele também destaca que existem também aqueles

\footnotetext{
${ }^{1}$ Tradução livre para Survival Copula. Veja Nelsen (2006), seção 2.6 páginas 32-33.
} 
que nomeiam por cópula de Gumbel-Hougaard (veja Hougaard, 1986 e Hutchinson e Lai, 1990). O motivo se deve a existência de outro membro arquimediano ser também batizado com o nome de Gumbel. Como esse texto não tem interesse nessa outra família membro, então o texto não cairá em confusão desde que fique claro que a cópula de Gumbel seja escrita como

$$
\begin{aligned}
K_{\alpha}(t, c) & =\exp \left\{-\left[(-\log t)^{\alpha}+(-\log c)^{\alpha}\right]^{\frac{1}{\alpha}}\right\}, \alpha \geq 1, \\
\varphi(t) & =(-\log t)^{\alpha}, \\
\tau & =\frac{\alpha}{1+\alpha},
\end{aligned}
$$

com pseudo-inversa

$$
\varphi^{-1}(t)=\mathrm{e}^{-t^{1 / \alpha}}
$$

- para $\alpha \geq 1$, então existe uma associação (concordância) positiva $\left(\tau>\frac{1}{2}\right)$; e

- obviamente também implica que o nível de dependência $\tau$ não pode ser zero.

O gerador da cópula de Gumbel também é um gerador estrito. Quanto ao grau de associação (concordância), o coeficiente $\tau$ de Kendall é maior ou igual a $\frac{1}{2}$. Portanto, deve-se levar em consideração esse fato quando existir suspeita de uma concordância nula ou negativa entre as variáveis, fazendo o uso da cópula de Gumbel inapropriada. As expressões analíticas das derivadas de até terceira ordem para cópula Gumbel estão disponíveis no Apêndice A.

\subsubsection{Gerador de uma amostra pseudoaleatória}

A seguir, destaca-se uma abordagem de Xu et al. (2018) para gerar uma amostra pseudoaleatória: primeiramente, o par $\left(S_{T}\left(x_{i}\right), S_{C}\left(x_{i}\right)\right)$ pode ser gerado por uma rotina previamente disponível no software R, por exemplo, com chamada copula: : frankCopula. São destacadas duas possibilidades:

(S1) (Condicional) Seja $g: \operatorname{dom} T \mid C \longrightarrow I$ função de distribuição de sobrevivência da variável aleatória (v.a.) contínua $T \mid C$. Uma transformação que mapeia um par $(X, Y)$ de v.a. contínuas seguindo distribuição uniforme 0 e 1 ao par $(T, C)$ de variáveis com cópula $K_{\alpha}: I^{2} \longrightarrow I$ é

$$
\begin{aligned}
& X \leftarrow T, \\
& Y \leftarrow g^{-1}(C \mid X) .
\end{aligned}
$$

(S2) (Inversa do Gerador). Seguindo a abordagem proposta em Embrechts et al. (2001), suponha que $\left(U_{1}, U_{2}\right)$ siga uma cópula arquimediana bidimensional com função geradora $\varphi(t)$. Então

(S2.1) gere independentemente $u_{1}$ e $u_{2}$ seguindo uniforme entre 0 e 1 ;

(S2.2) faça $t=C^{-1}\left(u_{2}\right), \operatorname{com} C(t)=t-\frac{\varphi(t)}{\varphi^{-1}(t)} ; \mathrm{e}$ 
(S2.3) por último, faça $u=\varphi^{-1}\left(u_{1} \varphi(t)\right)$ e $v=\varphi^{-1}\left(\left(1-u_{1}\right) \varphi(t)\right)$.

O esquema (S2) a cima pode ser uma alternativa para o caso (S1) quando se deseja obter expressão analítica sobre a distribuição de sobrevida marginal condicional de $T \mid C$. No presente texto, apenas foi utilizado o esquema (S1) para auxiliar na construção de réplicas no estudo de simulações presentes no Capítulo 4.

Para o esquema (S1), pode-se provar os resultados

$$
\begin{aligned}
& \operatorname{Pr}\{T>t \mid C=c\}=\lim _{\Delta c \longrightarrow 0} \frac{K(t, c+\Delta c)-K(t, c)}{\Delta c}=\left.\frac{\partial}{\partial y} K(t, y)\right|_{y=c}, \\
& \operatorname{Pr}\{C>c \mid T=t\}=\lim _{\Delta t \rightarrow 0} \frac{K(t+\Delta t, c)-K(t, c)}{\Delta t}=\left.\frac{\partial}{\partial x} K(x, c)\right|_{x=t} ^{\prime},
\end{aligned}
$$

lembrando o Teorema 2, o que torna o esquema (1) uma conveniente abordagem para gerar par aleatório. Vamos demonstrar o resultado (2.8) e deixar omitido a demonstração de (2.9) por ser análogo.

Demonstração. Seja o resultado (2.8). A seguir sua demonstração

$$
\begin{aligned}
P(T>t \mid C=c) & =\lim _{h \rightarrow 0} P(T>t \mid c \leq C<c+h) \\
& =\lim _{h \rightarrow 0} \frac{P(T>t, C>c)-P(T>t, C>c+h)}{P(C<c)-P(C<c+h)} \\
& =\lim _{h \rightarrow 0} \frac{P(T>t, C>c+h)-P(T>t, C>c)}{P(C<c+h)-P(C<c)} \\
& =\lim _{h \rightarrow 0} \frac{S_{T, C}(t, c+h)-S_{T, C}(t, c)}{S_{C}(c+h)-S(c)}, \quad \text { pelo Teorema de Sklar, } \\
& =\lim _{h \rightarrow 0} \frac{K\left(S_{T}(t), S_{C}(c+h)\right)-K\left(S_{T}(t), S_{C}(c)\right)}{S_{C}(c+h)-S(c)}, \quad \text { sendo } \Delta c=S_{C}(c+h)-S_{C}(c), \\
& =\lim _{\Delta c \rightarrow 0} \frac{K\left(S_{T}(t), S_{C}(c)+\Delta c\right)-K\left(S_{T}(t), S_{C}(c)\right)}{\Delta c} \\
& =\left.\frac{\partial}{\partial y} K(t, y)\right|_{y=c} .
\end{aligned}
$$




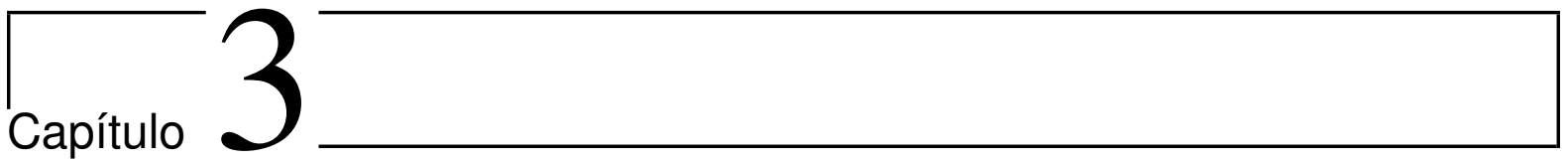

\section{Modelos de Taxas de Falha Proporcionais sob Censura Dependente}

Nesse presente capítulo são apresentados os modelos semiparamétricos de taxas de falha proporcionais assumindo que a possível estrutura de dependência acerca da censura à direita seja via uma função de cópula. A seção 3.4 apresenta o primeiro modelo que foi proposto por Xu et al. (2018). Aqui também é apresentada a construção do modelo proposto bem como suas respectivas funções de verossimilhança e log-verossimilhança, baseando-se na adaptação da função de verossimilhança (veja Wienke, 2010, páginas 43-50) para modelos de fragilidade. A seção 3.5 apresenta e discute a proposta desenvolvida por Huang e Zhang (2008). Entretanto, antes do texto prosseguir, é necessário uma explanação sobre dois conceitos importantes em modelos de Análise de Sobrevivência, a saber: censura independente e censura dependente.

\subsection{Estruturas de censura}

Essa presente seção é uma breve mas necessária passagem para compreender sobre os tipos de mecanismos (estruturas) de censura. Pretende-se discutir os conceitos de censura independente e censura dependente, tão fundamentais daqui em diante. Sejam as variáveis aleatórias $T$, que denota o tempo de falha; $A$, que denota o tempo de censura; e $Z$, que denota a variável preditora associada.

Para discutir a situação em que a censura é dependente ou informativa, faz-se necessário introduzir algumas formulações básicas. Para dados com censura à direita, é comum o indivíduo ter consigo a representação pelas seguintes variáveis aleatórias: o tempo de interesse (ou falha) $T$, frequentemente não-observável; o tempo observável $X$, que é o tempo de falha ou o tempo de censura ( $X$ também é muitas vezes chamado de tempo de acompanhamento); e o indicador $\Delta=I(T=X)$. Em outras palavras, $T$ representa o tempo decorrido entre a origem (pré-determinada) do ensaio até o tempo de um específico evento de interesse e $X$ representa o tempo decorrido entre a origem do ensaio até o desfecho observável, isto é, o evento de interesse ocorre $(\Delta=1)$ ou por alguma razão a observação é censurada $(\Delta=0)$. Para construir 
o que para Lagakos (1979) ficou denominado como modelo sob censura independente, é preciso reescrever o tempo observado como

$$
X=\min (T, C) \text { e } \quad \Delta=\left\{\begin{array}{ll}
1, & T \leq C \\
0, & \text { caso contrário }
\end{array} .\right.
$$

Caso $T$ e $A$ sejam condicionalmente independentes dado $Z$, então é dito que $A$ é censura independente. Isto é, caso no modelo a igualdade

$$
\operatorname{Pr}\left\{T \in E_{1}, A \in E_{2} \mid Z\right\}=\operatorname{Pr}\left\{T \in E_{1} \mid Z\right\} \operatorname{Pr}\left\{A \in E_{2} \mid Z\right\}
$$

for verdadeira, então é dito que o modelo tem estrutura de censura independente. Vale enfatizar que a preditora $Z$ não é necessariamente variável aleatória e, portanto, a notação $\operatorname{Pr}\{\cdot \mid Z\}$ é apenas para evidenciar que o modelo também está em função da variável preditora. Esse conceito de censura independente está bastante consolidado na literatura e não há confusão sobre essa ideia. A maioria dos modelos propostos na literatura em Análise de Sobrevivência são válidos sob a suposição de censura independente.

Em seu artigo, Lagakos assumiu que as distribuições de $T$ e $C$ sejam distribuições funcionalmente independentes. Kalbfleisch e Prentice (2002) definiram como censura não informativa essa condição de que a distribuição de $C$ não envolve parâmetros da distribuição de $T$. Contudo, Lagakos utilizou essa mesma terminologia para um outro conceito em seu artigo, que será discutido mais adiante.

Nesta sequência, é usado um modelo genérico para uma função de verossimilhança de uma amostra homogênea de observações independentes $\left(x_{i}, \delta_{i}\right)$ de $(X, \Delta)$ que é proporcional a

$$
\prod_{i}\left[f_{T}\left(x_{i}\right) S_{C}\left(x_{i}\right)\right]^{\delta_{i}}\left[f_{C}\left(x_{i}\right) S_{T}\left(x_{i}\right)\right]^{1-\delta_{i}}
$$

com $f_{T}(x)=-\frac{d}{d x} S_{T}(x), f_{C}(x)=-\frac{d}{d x} S_{C}(x)$, sendo $S_{T}(x)$ e $S_{C}(x)$ denotando a função de sobrevivência associada a $T$ e $C$, respectivamente, e $\delta_{i}=1$ se ocorreu falha. Com fácil manipulação algébrica, também é possível reescrever mais confortavelmente como

$$
\left\{\prod_{j} f_{T}\left(x_{j}\right) \prod_{k} s_{T}\left(x_{k}\right)\right\}\left\{\prod_{j} s_{C}\left(x_{j}\right) \prod_{k} f_{C}\left(x_{k}\right)\right\},
$$

com os $x_{j}$ representando os tempos das observações que falharam $\left(\delta_{i}=1\right)$ e os $x_{k}$ representando os tempos das observações que foram censuradas $\left(\delta_{i}=0\right)$. Note que as componentes dos produtos dentro de ambas chaves são distintas a respeito dos parâmetros (desconhecidos) porquê as funções de sobrevivência $S_{T}$ e $S_{C}$ são funcionalmente independentes, que é uma suposição. Então uma consequência para obter os estimadores de máxima verossimilhança do tempo de sobrevivência $T$ deste modelo é que as componentes da outra chave são absorvidas pela constante de proporcionalidade da função. 
Lagakos (1979) definiu uma outra classe de censura, a não-prognóstica. Utilizando a notação do autor, sejam $D_{u}$ e $C_{u}$ a ocorrência de uma observação não censurada e censurada, respectivamente. Segundo ele, um mecanismo de censura é não prognóstico se

$$
P\left(T \in N_{t} \mid C_{u}\right)=\frac{d F_{T}(t)}{1-F_{T}(u)}, t>u,
$$

em que $N_{t}=(t-d t, t)$ e $F_{T}(t)=1-S_{T}(t)$. O autor argumenta que a classe de censura independente está contida na classe de censura não-prognóstica. Além disso, o resultado de que função de verossimilhança é proporcional a

$$
\prod_{i}\left[f_{T}\left(x_{i}\right)\right]^{\delta_{i}}\left[S_{T}\left(x_{i}\right)\right]^{1-\delta_{i}}
$$

é válido para o caso de censura não-prognóstica e, portanto, também para censura independente sob a suposição de que a distribuição de $C$ é funcionalmente independente da distribuição de $T$. Por fim, Lagakos ainda discutiu o que chamou de censura não informativa. Esse é um conceito mais técnico e de difícil compreensão. A condição para que a censura seja não informativa é que $a(u)+B(u)=1$ quase certamente em $F_{T}(u)$, ou seja,

$$
\int_{0}^{\infty}(a(u)+B(u)) d F(u)=1
$$

em que $a(u)=P\left(\Delta=1 \mid T \in N_{u}\right)$ e $d B(u)=P\left(C_{u} \mid T>u\right)$. Williams e Lagakos (1977) observaram que " $a(u)$ representa a probabilidade de um indivíduo que falha em $u$ ter seu tempo de falha observado e $d B(u)$ é a probabilidade de um indivíduo que sobreviveu a $u$ ser censurado em $u^{\prime \prime}$. Eles denominaram essa condição de soma-constante e posteriormente Lagakos (1979) a denominou de censura não informativa. Segundo os autores, esse mecanismo de censura é o de maior dificuldade de interpretação, porém é matematicamente o mais geral. Os autores mostram que esse grupo de mecanismos de censura informativa contém a censura não-prognóstica. Os autores em ambos artigos argumentaram que a verossimilhança (3.1) é válida para qualquer situação de censura não informativa ou soma-constante.

Um extensa discussão foi feita por Lagakos (1979) sobre censura informativa, que seria o caso dos mecanismos de censura que não estão no grupo maior que ele denominou de censura não informativa. Alguns fenômenos que o autor destacou que podem levar a censura informativa são:

(3.1.1) um ensaio clínico em que alguns pacientes podem ser removidos do estudo por razões possivelmente relacionadas ao tratamento e, portanto, censurar o tempo de sobrevivência;

(3.1.2) um ensaio clínico em que os pacientes podem estar em estado de quadro grave como, por exemplo, em estágio de metástase e, por design de estudo, são removidos do estudo e assim seus respectivos tempos de sobrevivência não são mais relatados; e

(3.1.3) um ensaio clínico entre animais que podem sofrer falha por dois tipos de eventos mas 
somente um é de interesse, levando o segundo evento menos relevante à pesquisa ser induzido como um mecanismo de censura.

É importante ressaltar que o termo "censura dependente"não foi empregado por Lagakos. No entanto, o autor discutiu uma alternativa para análise de dados que não apresenta censura informativa é utilizar um modelo bivariado para $T$ e $C$, sem a suposição de independência. Segundo o autor, o problema dessa abordagem - que é a adotada neste trabalho - é que o modelo deve de ser declarado e, por conseguinte, não há como testar as imposições feitas sobre essa distribuição conjunta por questões de identificabilidade, que é uma grande crítica às abordagens de modelagem de censura dependente com cópulas.

\subsection{Problema de Identificabilidade}

Antes dos modelos serem apresentados, faz-se necessário explicar sobre o problema de não identificabilidade em modelos de Análise de Sobrevivência em conjunto de dados em que se assume censura informativa. O par observável e sua respectiva variável preditora não são informações suficientes para investigar a dependência entre o tempo de sobrevivência verdadeiro $T$ e o tempo de censura C. Essa constatação vem desde Tsiatis (1975) fundamentando os aspectos da ocorrência da não identificabilidade quando a premissa de censura não informativa é plausível em conjuntos de dados que existem mais de um evento de interesse que provoca a falha nas observações, esse quadro caindo sobre conjunto de dados sob riscos competitivos. Então não é incomum alguns autores renomearem modelos sob censura dependente como modelos de riscos competitivos.

Sobre sobrevivência bruta, Tsiatis (1975) a definiu como probabilidade do i-ésimo indivíduo sobreviver até o tempo $x$ e falhar por causa do $i$-ésimo risco. Originalmente, Tsiatis elaborou a função de sobrevivência bruta para um quadro com $k$ riscos para aquele indivíduo mas na proposta deste texto com três funções de sobrevivência bruta é reescrita como: (3.2.1) a probabilidade daquele $i$-ésimo indivíduo falha em $x$ e ser censurado após $x$; e (3.2.2) a probabilidade daquele $i$-ésimo indivíduo ser censurado em $x$ e ser falha após $x$. O propósito é estimar essas funções de sobrevivência bruta por meio de uma função conjunta de probabilidade dos tempos de sobrevivência com seus respectivos riscos. Tsiatis demonstrou que, com ou sem a premissa de independência entre os tempos de sobrevivência, a função conjunta implica em funções brutas iguais resultando em uma não identificabilidade. Exposto essa condição, Huang e Zhang (2008) disseram que o melhor caminho seria uma análise sensitiva como em Zheng e Klein (1995), que assumiram uma função de cópula dentro da modelagem para dados sob riscos competitivos estimando as funções marginais pela abordagem não paramétrica. Ademais, a aplicação de cópula em modelos de análise de sobrevivência sob censura informativa provavelmente se tornou comum por possibilitar um caminho para estudar explicitamente a estrutura de dependência por intermédio do parâmetro $\alpha$ da função de cópula em função de algum coeficiente de dependência como, por exemplo, a medida $\tau$ de Kendall.

Diante dessa nova abordagem em análise de sobrevivência sob censura informativa em 
que se propõe uma determinada cópula, Wang (2012) levantou a seguinte questão: dado um conjunto de dados sob censura informativa, é possível determinar o parâmetro $\alpha$ da cópula? Em outras palavras, o interesse é responder se o conjunto de dados contém informação suficiente para identificar a dependência do tempo de sobrevivência $T$ e o tempo de censura $C$. Então Wang (2012) provou que diferentes cópulas sob diferentes níveis de $\tau$ podem levar a mesma distribuição para $(X, \Delta)$ em um conjunto de dados sob censura informativa. Sendo assim, persiste o problema de não identificabilidade para modelos sob censura dependente baseados na abordagem de cópula cujo conjunto de dados estão sob censura informativa. Nesse texto, não foi estudado nenhum trabalho em que se apresente uma alternativa a esse problema senão pela análise sensitiva do coeficiente $\tau$. Em outros contextos como em modelos de fragilidade (veja Chandra, 2015) e modelos de riscos semi-competitivos (veja Fine et al., 2001) o problema de não identificabilidade é contornável sob certas condições de regularidade.

\subsection{Modelo Paramétrico}

A presente seção apresenta nossa contribuição paramétrica proposta como alternativa aos modelos propostos por $\mathrm{Xu}$ et al. (2017) com abordagem não paramétrica e Xu et al. (2018) com abordagem semiparamétrica. Para tanto, usa-se a distribuição de Weibull com função de sobrevivência $S(x)=\mathrm{e}^{-(x / \lambda)^{\alpha}}$, sendo $\alpha>0$ parâmetro de forma e $\lambda>0$ parâmetro de escala. Sendo assim, apresentamos o modelo paramétrico Weibull-Weibull.

Antes da continuação da construção da abordagem paramétrica, faz-se necessário explicar como foi proposta e construída a função de verossimilhança. Essa construção é escrita a partir de um $i$-ésimo indivíduo que possui as informações efetivamente observáveis $\left(x_{i}, \delta_{i T}, \delta_{i C}, \mathbf{Z}_{i}^{\top}\right)$, com $x_{i}$ o tempo observado; seus respectivos status de falha $\delta_{i T}$ e censura $\delta_{i C}$; e $Z_{i}$ vetor de covariáveis. Quando o $i$-ésimo indivíduo possuir status $\delta_{i T}=\delta_{i C}=0$, então é dito que seu status é de censura independente. Huang e Zhang (2008) referiram esse último indivíduo como um indivíduo que contém uma observação administradamente censurada. A seguir, são destacadas algumas nomeações que perdurarão no decorrer do texto, em especial na seção 3.4 .

Sejam $T=\left\{T_{i}: i \in \Gamma\right\}$ e $C=\left\{C_{i}: i \in \Gamma\right\}$ os conjuntos das variáveis aleatórias do tempo de falha e censura, indexados pelo conjunto $\Gamma \subset \mathbb{N}$. O interesse é no fenômeno da censura à direita, $C_{i}$, que pode ser dependente do tempo de falha $T_{i}$. Descreve-se aqui a abordagem proposta por $\mathrm{Xu}$ et al. (2018) com o tempo de sobrevida observável $X_{i}=\min \left(T_{i}, C_{i}, A_{i}\right), \operatorname{com} A_{i}$ como tempo de censura independente do $i$-ésimo indivíduo em estudo. Eles explanam, então, que a informação observável do $i$-ésimo indivíduo é escrita como $\left(X_{i}, \Delta_{i T}, \Delta_{i C}, \mathbf{Z}_{i}^{\top}\right)$, com o indicador da ocorrência do evento de interesse $\Delta_{i T}=\Delta_{T}\left(x_{i}\right)=I\left(X_{i}=T_{i}\right)$, indicador da ocorrência de censura dependente $\Delta_{i C}=\Delta_{C}\left(x_{i}\right)=I\left(X_{i}=C_{i}\right)$ e $\mathbf{Z}_{i}$ vetor de covariáveis associadas de ordem $p \times 1$. Os dados efetivamente observados para o $i$-ésimo indivíduo são $\left(x_{i}, \Delta_{i T}, \Delta_{i C}, \mathbf{Z}_{i}^{\top}\right)$. Aqui é assumido que os indivíduos são independentes entre si.

Para a distribuição marginal do tempo de falha, assume-se um modelo de taxas de falha proporcionais:

$$
\lambda_{T}(t \mid \mathbf{Z})=\lambda_{0 T}(t) g(t \mid \mathbf{Z}), \quad t>0,
$$


sendo, em Colosimo e Giolo (2006), a componente $\lambda_{0 T}(t)$ uma função comumente denominada de função de taxas de falha de base como também e usualmente chamada de função basal. A componente $g(t \mid \mathbf{Z})$ é uma função não negativa que especifica a relação entre as covariáveis $\mathbf{Z}$ e a taxas de falha com tal sorte que essa função deve obedecer $g(t \mid \mathbf{0})=1$, o que implica $\lambda_{T}(t)=\lambda_{0 T}(t)$. Como o texto apresenta um estudo sob censura dependente, é necessário também introduzir o modelo de taxas de censura

$$
\lambda_{C}(c \mid \mathbf{Z})=\lambda_{0 C}(c) g(c \mid \mathbf{Z}), \quad c>0,
$$

sendo a componente $\lambda_{0 C}(c)$ uma função de taxas de censura de base. Analogo a função a $g(t \mid \mathbf{Z})$, a componente $g(c \mid \mathbf{Z})$ é uma função não negativa que especifica a relação entre as covariáveis $\mathbf{Z}$ e a taxas de censura.

Admitindo que a funções de ligação sejam $g(t \mid \mathbf{Z})=\mathrm{e}^{\mathbf{Z}^{\top} \boldsymbol{\beta}} \mathrm{e} g(c \mid \mathbf{Z})=\mathrm{e}^{\mathbf{Z}^{\top} \boldsymbol{\phi}}$, os modelos de taxas de falha e censura resultam em

$$
\begin{aligned}
& \lambda_{T}(t \mid \mathbf{Z})=\lambda_{0 T}(x) \mathrm{e}^{\mathbf{Z}^{\top} \boldsymbol{\beta}}, \\
& \lambda_{C}(c \mid \mathbf{Z})=\lambda_{0 C}(x) \mathrm{e}^{\mathbf{Z}^{\top} \boldsymbol{\phi}},
\end{aligned}
$$

$\operatorname{com} \boldsymbol{\beta}=\boldsymbol{\beta}_{p \times 1}$ e $\boldsymbol{\phi}=\phi_{p \times 1}$ vetores dos coeficientes de regressão para as funções de taxa associadas ao tempo de falha $T$ e ao tempo de censura $C$, respectivamente, e as funções não negativas $\lambda_{0 T}(t)$ e $\lambda_{0 C}(c)$ são as funções basais. Denotam-se as funções basais acumuladas por $\Lambda_{0 T}(t)$ e $\Lambda_{0 C}(c)$, respectivamente. O principal objetivo é a obtenção das estimativas, $\hat{\boldsymbol{\beta}}$ e $\widehat{\lambda_{0 T}}(t)$, dos vetores de coeficientes de regressão $\beta$ e a função basal $\lambda_{0 T}(t)$ mesmo assumindo que as covariáveis são para o evento de interesse e censura dependente. Por outro lado, a estimação dos parâmetros $\phi$ e $\lambda_{0 c}(c)$ não podem ser evitada devido ao mecanismo de dependência nos modelos. Por fim, antes de enunciar os modelos, enfatiza-se neste texto que não se assumirá $T$ e $C$ independentes mas que, tão somente, sua conjunta será obtida por meio de uma das três famílias membros da classe arquimediana discutidas no Capítulo 2, ou seja, a distribuição conjunta de $T$ e $C$ é dada por

$$
S_{T, C}(t, c)=K\left(S(t), S_{C}(c)\right)
$$

em que $K(t, c)$ é uma função de cópula com parâmetro $\alpha$ fixado - sendo predeterminado devido ao problema de não identificabilidade incluso em modelagens que adotam abordagem via cópula.

Agora sejam $f_{T}(t), f_{C}(c)$ e $f_{A}(a)$ as funções densidade de probabilidade e suas respectivas funções de sobrevivência $S_{T}(t), S_{C}(c)$ e $S_{A}(\cdot)$ referentes ao tempo de falha, tempo de censura dependente e tempo de censura independente. Seja $\mathbf{Z}=\left(\mathbf{Z}_{1}^{\top}, \ldots, \mathbf{Z}_{n}^{\top}\right)^{\top}$ a matriz de covariáveis e $\eta=(\beta, \phi)^{\top}$ vetor completo dos coeficientes de regressão para as funções de taxas de falha associadas aos tempos de sobrevivência $T$ e ao tempo de censura $C$. Cada indivíduo $i$ possui uma, e somente uma, contribuição na função de verossimilhança. Considere inicialmente a 
contribuição de um indivíduo para o qual a falha foi observada:

$$
\begin{aligned}
L_{i T} & =\operatorname{Pr}\left(T=x_{i}, C>x_{i}, A>x_{i} \mid \mathbf{Z}_{i}\right), \quad \text { por independência e omitindo a condicionalidade } \\
& =\operatorname{Pr}\left(T=x_{i}, C>x_{i}\right) \operatorname{Pr}\left(A>x_{i}\right) \\
& =\operatorname{Pr}\left(C>x_{i} \mid T=x_{i}\right) \operatorname{Pr}\left(T=x_{i}\right) S_{A}\left(x_{i}\right) \\
& =\operatorname{Pr}\left(C>x_{i} \mid T=x_{i}\right) f_{T}\left(x_{i}\right) S_{A}\left(x_{i}\right), \quad \text { lembrando do resultado (2.9) } \\
& =\left.\frac{\partial}{\partial u} K\left(S_{T}(u), S_{C}\left(x_{i}\right)\right)\right|_{u=x_{i}} f_{T}\left(x_{i}\right) S_{A}\left(x_{i}\right) \\
& =f_{T}\left(x_{i}\right) K_{1}\left(S_{T}\left(x_{i}\right), S_{C}\left(x_{i}\right)\right) S_{A}\left(x_{i}\right)
\end{aligned}
$$

em que a notação $K_{1}(t, c)=\left.\frac{\partial}{\partial x} K(x, c)\right|_{x=t}$. Analogamente, para um indivíduo com censura dependente, tem-se:

$$
\begin{aligned}
L_{i C} & =\operatorname{Pr}\left(T>x_{i}, C=x_{i}, A>x_{i} \mid \mathbf{Z}_{i}\right), \quad \text { por independência e omitindo a condicionalidade } \\
& =\operatorname{Pr}\left(T>x_{i}, C=x_{i}\right) \operatorname{Pr}\left(A>x_{i}\right) \\
& =\operatorname{Pr}\left(T>x_{i} \mid C=x_{i}\right) \operatorname{Pr}\left(C=x_{i}\right) S_{A}\left(x_{i}\right) \\
& \left.=\operatorname{Pr}\left(T>x_{i} \mid C=x_{i}\right) f_{C}\left(x_{i}\right) S_{A}\left(x_{i}\right), \quad \text { lembrando do resultado } 2.8\right) \\
& =\left.\frac{\partial}{\partial v} K\left(S_{T}\left(x_{i}\right), S_{C}(v)\right)\right|_{v=x_{i}} f_{C}\left(x_{i}\right) S_{A}\left(x_{i}\right) \\
& =f_{C}\left(x_{i}\right) K_{2}\left(S_{T}\left(x_{i}\right), S_{C}\left(x_{i}\right)\right) S_{A}\left(x_{i}\right)
\end{aligned}
$$

sendo a notação $K_{2}(t, c)=\left.\frac{\partial}{\partial y} K(x, y)\right|_{y=c}$. Por fim, para um indivíduo com censura independente, tem-se:

$$
\begin{aligned}
L_{i A} & =\operatorname{Pr}\left(T>x_{i}, C>x_{i}, A=x_{i} \mid \mathbf{Z}_{i}\right), \quad \text { por independência e omitindo a condicionalidade } \\
& =\operatorname{Pr}\left(T>x_{i}, C>x_{i}\right) \operatorname{Pr}\left(A=x_{i}\right), \quad \text { lembrando que } T \text { e } C \text { são variáveis aleatórias contínuas } \\
& =f_{A}\left(x_{i}\right) K\left(S_{T}\left(x_{i}\right), S_{C}\left(x_{i}\right)\right) .
\end{aligned}
$$

Desse modo, a contribuição final para o $i$-ésimo indivíduo pode ser escrita como

$$
L_{i}=L_{i T}^{\delta_{i T}} L_{i C}^{\delta_{i C}} L_{i A}^{1-\delta_{i T}-\delta_{i C}}
$$

Portanto, a função de verossimilhança baseada em uma amostra de tamanho $n$ é

$$
\Phi(\boldsymbol{\eta} \mid \boldsymbol{x}, \mathbf{Z})=\prod_{i=1}^{n} L_{i T}^{\delta_{i T}} L_{i C}^{\delta_{i C}} L_{i A}^{1-\delta_{i T}-\delta_{i C}},
$$


com

$$
\left\{\begin{array}{rl}
L_{i T} & =f_{T}\left(x_{i}\right) K_{1}\left(S_{T}\left(x_{i}\right), S_{C}\left(x_{i}\right)\right) S_{A}\left(x_{i}\right) \\
L_{i C} & =f_{C}\left(x_{i}\right) K_{2}\left(S_{T}\left(x_{i}\right), S_{C}\left(x_{i}\right)\right) S_{A}\left(x_{i}\right) \\
L_{i A} & =f_{A}\left(x_{i}\right) K\left(S_{T}\left(x_{i}\right), S_{C}\left(x_{i}\right)\right)
\end{array} .\right.
$$

Observando que existem componentes que serão absorvidas pela constante de proporcionalidade de (3.3), será apresentado como escrever a função de log-verossimilhança absorvendo aquelas componentes $f_{A}\left(x_{i}\right)$ e $S_{A}\left(x_{i}\right)$. Observe que

$$
\begin{aligned}
\Phi_{l}(\eta \mid x, \mathbf{Z}) & =\log (\Phi(\eta \mid x, \mathbf{Z})) \\
& =\log \left(\prod_{i=1}^{n} L_{i T}^{\delta_{i T}} L_{i C}^{\delta_{i C}} L_{i A}^{1-\delta_{i T}-\delta_{i C}}\right) \\
& =\sum_{i=1}^{n} \delta_{i T} l_{i T}+\delta_{i C} l_{i C}+\left(1-\delta_{i T}-\delta_{i C}\right) l_{i A}
\end{aligned}
$$

com

$$
\left\{\begin{array}{l}
l_{i T}=\log L_{i T}=\log \left(f_{T}\left(x_{i}\right) K_{1}\left(S_{T}\left(x_{i}\right), S_{C}\left(x_{i}\right)\right) S_{A}\left(x_{i}\right)\right) \\
l_{i C}=\log L_{i C}=\log \left(f_{C}\left(x_{i}\right) K_{2}\left(S_{T}\left(x_{i}\right), S_{C}\left(x_{i}\right)\right) S_{A}\left(x_{i}\right)\right) \\
l_{i A}=\log L_{i A}=\log \left(f_{A}\left(x_{i}\right) K\left(S_{T}\left(x_{i}\right), S_{C}\left(x_{i}\right)\right)\right)
\end{array} .\right.
$$

Nas componentes $l_{i T}, l_{i C}$ e $l_{i A}$ são absorvidas pela constante de proporcionalidade as porções $f_{A}\left(x_{i}\right)$ e $S_{A}\left(x_{i}\right)$. Note que pode-se reescrever confortavelmente como segue

$$
\begin{aligned}
l_{i T} & =\log \left(f_{T}\left(x_{i}\right) K_{1}\left(S_{T}\left(x_{i}\right), S_{C}\left(x_{i}\right)\right) S_{A}\left(x_{i}\right)\right) \\
& =\log \left(\lambda_{T}\left(x_{i}\right) S_{T}\left(x_{i}\right) K_{1}\left(S_{T}\left(x_{i}\right), S_{C}\left(x_{i}\right)\right) S_{A}\left(x_{i}\right)\right) \\
& =\log \lambda_{T}\left(x_{i}\right)+\log S_{T}\left(x_{i}\right)+\log K_{1}\left(S_{T}\left(x_{i}\right), S_{C}\left(x_{i}\right)\right)+\log S_{A}\left(x_{i}\right) \\
& \propto \log \lambda_{T}\left(x_{i}\right)-\Lambda_{T}\left(x_{i}\right)+\log K_{1}\left(S_{T}\left(x_{i}\right), S_{C}\left(x_{i}\right)\right),
\end{aligned}
$$

que explicita a expressão proporcional resultante (3.4) como

$$
l_{i T}=\log \lambda_{0 T}\left(x_{i}\right)+\mathbf{Z}_{i}^{\top} \boldsymbol{\beta}-\Lambda_{T}\left(x_{i}\right)+\log K_{1}\left(\mathrm{e}^{-\Lambda_{T}\left(x_{i}\right)}, \mathrm{e}^{-\Lambda_{C}\left(x_{i}\right)}\right) .
$$

De forma análoga, tem-se

$$
\begin{aligned}
& l_{i C}=\log \lambda_{0 C}\left(x_{i}\right)+\mathbf{Z}_{i}^{\top} \boldsymbol{\phi}-\Lambda_{C}\left(x_{i}\right)+\log K_{2}\left(\mathrm{e}^{-\Lambda_{T}\left(x_{i}\right)}, \mathrm{e}^{-\Lambda_{C}\left(x_{i}\right)}\right), \\
& l_{i A}=\log K\left(\mathrm{e}^{-\Lambda_{T}\left(x_{i}\right)}, \mathrm{e}^{-\Lambda_{C}\left(x_{i}\right)}\right) .
\end{aligned}
$$

Para equação (3.4), as funções de sobrevivência dos tempos de falha $T$ e censura $C$ são definidas como $S_{T}(x):=e^{-\Lambda_{T}(x)}$ e $S_{C}(x):=e^{-\Lambda_{C}(x)}$, respectivamente. As funções de taxas de falha acumuladas são definidas como $\Lambda_{T}(x):=\Lambda_{0 T}(x) e^{\mathbf{Z}^{\top} \boldsymbol{\beta}} \mathrm{e} \Lambda_{C}(x):=\Lambda_{0 C}(x) e^{\mathbf{Z}^{\top} \boldsymbol{\phi}}, \operatorname{com} \Lambda_{0 T}(x):=$ $\int_{0}^{x} \lambda_{0 T}(w) d w$ e $\Lambda_{0 C}(x):=\int_{0}^{x} \lambda_{0 C}(w) d w$. Como as quantidades $f_{A}(\cdot)$ e $S_{A}(\cdot)$ pertencem a constante 
de proporcionalidade da função de verossimilhança com respeito ao parâmetro $\eta$, então podese extraí-las da equação (3.3) quando o objetivo for maximizá-la com relação $\eta$. É importante enfatizar que o parâmetro de dependência $\alpha$ da cópula não é componente de $\eta$. Por problema de não identificabilidade já discutido, esse parâmetro não pode ser estimado e a verossimilhança só pode ser obtida fixando um valor para $\alpha$ (ou $\tau$ ).

Em resumo e com certo abuso de notação, uma forma proporcional e explícita a (3.4) é escrita como

$$
\Phi_{l}(\eta \mid x, \mathbf{Z})=\sum_{i=1}^{n} \delta_{i T} l_{i T}+\delta_{i C} l_{i C}+\left(1-\delta_{i T}-\delta_{i C}\right) l_{i A}
$$

com

$$
\left\{\begin{array}{l}
l_{i T}=\log \lambda_{0 T}\left(x_{i}\right)+\mathbf{Z}_{i}^{\top} \boldsymbol{\beta}-\Lambda_{0 T}\left(x_{i}\right) \mathrm{e}^{\mathbf{Z}_{i}^{\top} \boldsymbol{\beta}}+\log K_{1}\left(\mathrm{e}^{-\Lambda_{0 T}\left(x_{i}\right) \mathrm{e}_{i}^{\mathbf{Z}_{i}^{\top} \boldsymbol{\beta}}}, \mathrm{e}^{-\Lambda_{0 C}\left(x_{i}\right) \mathrm{e}_{i}^{\mathbf{Z}_{i}^{\top} \phi}}\right) \\
l_{i C}=\log \lambda_{0 C}\left(x_{i}\right)+\mathbf{Z}_{i}^{\top} \boldsymbol{\phi}-\Lambda_{0 C}\left(x_{i}\right) \mathrm{e}^{\mathbf{Z}_{i}^{\top} \boldsymbol{\phi}}+\log K_{2}\left(\mathrm{e}^{-\Lambda_{0 T}\left(x_{i}\right) \mathrm{e}_{i}^{\mathbf{Z}_{i}^{\top} \boldsymbol{\beta}}}, \mathrm{e}^{-\Lambda_{0 C}\left(x_{i}\right) \mathrm{e}_{i}^{\top} \boldsymbol{\phi}}\right) \\
l_{i A}=\log K\left(\mathrm{e}^{-\Lambda_{0 T}\left(x_{i}\right) \mathrm{e}^{\mathbf{Z}_{i}^{\top} \boldsymbol{\beta}}}, \mathrm{e}^{-\Lambda_{0 C}\left(x_{i}\right) \mathrm{e}^{\mathbf{Z}_{i}^{\top} \phi}}\right)
\end{array} .\right.
$$

Esse último resultado é útil para obter o gradiente e matriz hessiana. Vale lembrar que os resultados a cima serão utilizados para ambas abordagens paramétrica e semiparamétrica, respectivamente. A seguir, são apresentadas as derivadas parciais com respeito a $\beta_{j}$ e $\phi_{j}$ para (3.5):

$$
\left\{\begin{aligned}
\frac{\partial}{\partial \beta_{j}} \Phi_{l}(\eta \mid \mathbf{Z}) & =\sum_{i=1}^{n}\left\{\delta_{i T}-\delta_{i T} \Lambda_{T}\left(x_{i}\right)-H_{1}\left(x_{i}\right) S_{T}\left(x_{i}\right) \Lambda_{T}\left(x_{i}\right)\right\} Z_{i j} \\
\frac{\partial}{\partial \phi_{j}} \Phi_{l}(\eta \mid \mathbf{Z}) & =\sum_{i=1}^{n}\left\{\delta_{i C}-\delta_{i C} \Lambda_{C}\left(x_{i}\right)-H_{2}\left(x_{i}\right) S_{C}\left(x_{i}\right) \Lambda_{C}\left(x_{i}\right)\right\} Z_{i j}
\end{aligned}\right.
$$

com

$$
H_{1}\left(x_{i}\right)=\delta_{i T} \frac{K_{11}\left(S_{T}\left(x_{i}\right), S_{C}\left(x_{i}\right)\right)}{K_{1}\left(S_{T}\left(x_{i}\right), S_{C}\left(x_{i}\right)\right)}+\delta_{i C} \frac{K_{12}\left(S_{T}\left(x_{i}\right), S_{C}\left(x_{i}\right)\right)}{K_{2}\left(S_{T}\left(x_{i}\right), S_{C}\left(x_{i}\right)\right)}+\left(1-\delta_{i T}-\delta_{i C}\right) \frac{K_{1}\left(S_{T}\left(x_{i}\right), S_{C}\left(x_{i}\right)\right)}{K\left(S_{T}\left(x_{i}\right), S_{C}\left(x_{i}\right)\right)}
$$

$\mathrm{e}$

$$
H_{2}\left(x_{i}\right)=\delta_{i C} \frac{K_{22}\left(S_{T}\left(x_{i}\right), S_{C}\left(x_{i}\right)\right)}{K_{2}\left(S_{T}\left(x_{i}\right), S_{C}\left(x_{i}\right)\right)}+\delta_{i T} \frac{K_{21}\left(S_{T}\left(x_{i}\right), S_{C}\left(x_{i}\right)\right)}{K_{1}\left(S_{T}\left(x_{i}\right), S_{C}\left(x_{i}\right)\right)}+\left(1-\delta_{i T}-\delta_{i C}\right) \frac{K_{2}\left(S_{T}\left(x_{i}\right), S_{C}\left(x_{i}\right)\right)}{K\left(S_{T}\left(x_{i}\right), S_{C}\left(x_{i}\right)\right)} .
$$

Ainda é possível escrever em uma forma mais compacta usando a notação matricial a seguir

$$
\begin{cases}\frac{\partial}{\partial \beta} \Phi_{l}(\eta \mid \mathbf{Z}) & =\mathbf{Z}^{\top}\left(\boldsymbol{\delta}_{T}-\mathbf{A}_{1} \mathbf{1}_{n}\right) \\ \frac{\partial}{\partial \phi} \Phi_{l}(\eta \mid \mathbf{Z}) & =\mathbf{Z}^{\top}\left(\boldsymbol{\delta}_{C}-\mathbf{A}_{2} \mathbf{1}_{n}\right)\end{cases}
$$

$\operatorname{com} \boldsymbol{\delta}_{T}=\left(\delta_{1 T}, \ldots, \delta_{n T}\right)^{\top}, \boldsymbol{\delta}_{C}=\left(\delta_{1 C}, \ldots, \delta_{n C}\right)^{\top}, \mathbf{A}_{1}=\operatorname{diag}\left(\delta_{i T} \Lambda_{T}\left(x_{i}\right)+H_{1}\left(x_{i}\right) S_{T}\left(x_{i}\right) \Lambda_{T}\left(x_{i}\right)\right), \mathbf{A}_{2}=$ $\operatorname{diag}\left(\delta_{i C} \Lambda_{C}\left(x_{i}\right)+H_{2}\left(x_{i}\right) S_{C}\left(x_{i}\right) \Lambda_{C}\left(x_{i}\right)\right)$ e $\mathbf{1}_{n}=(1, \ldots, 1)^{\top}$ vetor um de tamanho $n$. Então, o gradiente 
dos coeficientes de regressão $\eta_{1}=(\beta, \phi)^{\top}$ resultante é

$$
\frac{\partial}{\partial \eta_{1}} \Phi_{l}(\eta \mid \mathbf{Z})=\left(\begin{array}{c}
\frac{\partial}{\partial \beta} \Phi_{l}(\eta \mid \mathbf{Z}) \\
\frac{\partial}{\partial \phi} \Phi_{l}(\eta \mid \mathbf{Z})
\end{array}\right) .
$$

Os resultados (3.6 3.8) estão disponíveis no material suplementar em Xu et al. (2018) mas sem demonstrações. Então estão disponíveis as demonstrações no Apêndice B.

Para desenvolver o modelo paramétrico, então sejam $T \sim \operatorname{Weibull}\left(\alpha_{t}, \lambda_{t}\right)$ e $C \sim \operatorname{Weibull}\left(\alpha_{c}, \lambda_{c}\right)$. Como consequência, as componentes da função de log-verossimilhança (3.5) são escritas como

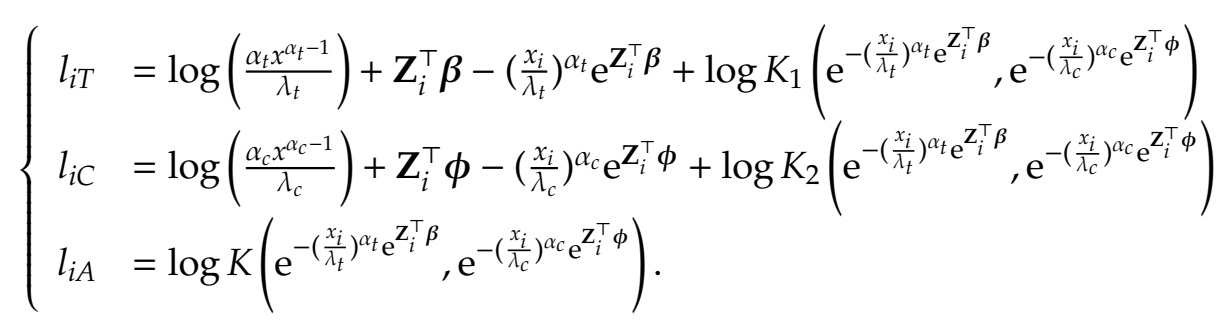

Agora com um modelo de 4 parâmetros a mais, esse modelo é útil para dar mais liberdade quanto a natureza (forma) da função basal. Por exemplo, para $\alpha_{t}=3$, tem-se um modelo que função basal quadrada. A seguir, são apresentadas as derivadas parciais de primeira ordem com relação a $\alpha_{t}$ das componentes da função de log-verossimilhança:

$$
\left\{\begin{aligned}
\frac{\partial}{\partial \alpha_{t}} l_{i T} & =\frac{1}{\alpha_{t}}+\left\{1-\left[\Lambda_{T}\left(x_{i}\right)+\frac{K_{11}\left(S_{T}\left(x_{i}\right), S_{C}\left(x_{i}\right)\right)}{K_{1}\left(S_{T}\left(x_{i}\right), S_{C}\left(x_{i}\right)\right)} S_{T}\left(x_{i}\right) \Lambda_{T}\left(x_{i}\right)\right]\right\} \log \left(\frac{x_{i}}{\lambda_{t}}\right) \\
\frac{\partial}{\partial \alpha_{t}} l_{i C} & =-\frac{K_{12}\left(S_{T}\left(x_{i}\right), S_{C}\left(x_{i}\right)\right)}{K_{2}\left(S_{T}\left(x_{i}\right), S_{C}\left(x_{i}\right)\right)} S_{T}\left(x_{i}\right) \Lambda_{T}\left(x_{i}\right) \log \left(\frac{x_{i}}{\lambda_{t}}\right) \\
\frac{\partial}{\partial \alpha_{t}} l_{i A} & =-\frac{K_{1}\left(S_{T}\left(x_{i}\right), S_{C}\left(x_{i}\right)\right)}{K\left(S_{T}\left(x_{i}\right), S_{C}\left(x_{i}\right)\right)} S_{T}\left(x_{i}\right) \Lambda_{T}\left(x_{i}\right) \log \left(\frac{x_{i}}{\lambda_{t}}\right)
\end{aligned}\right.
$$

Sobre as derivadas parciais, é mais conveniente escrevê-las em forma matricial do que em sua forma extensa. Veja as derivadas parciais de primeira ordem com relação a $\lambda_{t}$ das componentes da função de log-verossimilhança:

$$
\left\{\begin{aligned}
\frac{\partial}{\partial \lambda_{t}} l_{i T} & =-\frac{\alpha_{t}}{\lambda_{t}}+\left[\Lambda_{T}\left(x_{i}\right)+\frac{K_{11}\left(S_{T}\left(x_{i}\right), S_{C}\left(x_{i}\right)\right)}{K_{1}\left(S_{T}\left(x_{i}\right), S_{C}\left(x_{i}\right)\right)} S_{T}\left(x_{i}\right) \Lambda_{T}\left(x_{i}\right)\right]\left(\frac{\alpha_{t}}{\lambda_{t}}\right) \\
\frac{\partial}{\partial \lambda_{t}} l_{i C} & =\frac{K_{12}\left(S_{T}\left(x_{i}\right), S_{C}\left(x_{i}\right)\right)}{K_{2}\left(S_{T}\left(x_{i}\right), S_{C}\left(x_{i}\right)\right)} S_{T}\left(x_{i}\right) \Lambda_{T}\left(x_{i}\right)\left(\frac{\alpha_{t}}{\lambda_{t}}\right) \\
\frac{\partial}{\partial \lambda_{t}} l_{i A} & =\frac{K_{1}\left(S_{T}\left(x_{i}\right), S_{C}\left(x_{i}\right)\right)}{K\left(S_{T}\left(x_{i}\right), S_{C}\left(x_{i}\right)\right)} S_{T}\left(x_{i}\right) \Lambda_{T}\left(x_{i}\right)\left(\frac{\alpha_{t}}{\lambda_{t}}\right)
\end{aligned}\right.
$$

As expressões das derivadas parciais de primeira ordem das componentes de (3.5) com relação a $\alpha_{c}$ e também $\lambda_{c}$ são idênticas as expressões em (3.9-3.10), excetuando agora os índices te $T$ (apenas alterando $t$ e $T$ por $c$ e $C$ já é suficiente) e a ordem das derivadas parciais da cópula (a ordem $K_{i j}$ agora é $K_{j i}$ ). Portanto, optou-se por não explicitá-las. Em troca, é conveniente reescrever a soma das componentes em (3.9-3.10) da derivada de (3.5) na forma matricial. Seja 
$\eta_{2}=\left(\alpha_{t}, \lambda_{t}, \alpha_{c}, \lambda_{c}\right)$ o vetor de parâmetros da funções basais em (3.2), então segue o gradiente:

$$
\frac{\partial}{\partial \eta_{2}} \Phi_{l}(\eta \mid \mathbf{Z})=\left(\begin{array}{c}
\frac{1}{\alpha_{t}}\left(\boldsymbol{\delta}_{T}+\mathbf{A}_{1} \mathbf{1}_{n}\right)^{\top} \mathbf{1}_{n} \\
-\frac{\alpha_{t}}{\lambda_{t}}\left(\boldsymbol{\delta}_{T}-\mathbf{A}_{2} \mathbf{1}_{n}\right)^{\top} \mathbf{1}_{n} \\
\frac{1}{\alpha_{c}}\left(\boldsymbol{\delta}_{C}+\mathbf{B}_{1} \mathbf{1}_{n}\right)^{\top} \mathbf{1}_{n} \\
-\frac{\alpha_{c}}{\lambda_{c}}\left(\boldsymbol{\delta}_{C}-\mathbf{B}_{2} \mathbf{1}_{n}\right)^{\top} \mathbf{1}_{n}
\end{array}\right),
$$

com

$$
\begin{aligned}
& \boldsymbol{\delta}_{T}=\left(\delta_{1 T}, \ldots, \delta_{n T}\right)^{\top}, \\
& \boldsymbol{\delta}_{C}=\left(\delta_{1 C}, \ldots, \delta_{n C}\right)^{\top}, \\
& \mathbf{A}_{1}=\operatorname{diag}\left(\left[\delta_{i T}-\left(\Lambda_{T}\left(x_{i}\right) \delta_{i T}+H_{1}\left(x_{i}\right) S_{T}\left(x_{i}\right) \Lambda_{T}\left(x_{i}\right)\right)\right] \log \Lambda_{0 T}\left(x_{i}\right)\right), \\
& \mathbf{A}_{2}=\operatorname{diag}\left(\Lambda_{T}\left(x_{i}\right) \delta_{i T}+H_{1}\left(x_{i}\right) S_{T}\left(x_{i}\right) \Lambda_{T}\left(x_{i}\right)\right), \\
& \mathbf{B}_{1}=\operatorname{diag}\left(\left[\delta_{i C}-\left(\Lambda_{C}\left(x_{i}\right) \delta_{i C}+H_{2}\left(x_{i}\right) S_{C}\left(x_{i}\right) \Lambda_{C}\left(x_{i}\right)\right)\right] \log \Lambda_{0 C}\left(x_{i}\right)\right), \\
& \mathbf{B}_{2}=\operatorname{diag}\left(\Lambda_{C}\left(x_{i}\right) \delta_{i C}+H_{2}\left(x_{i}\right) S_{C}\left(x_{i}\right) \Lambda_{C}\left(x_{i}\right)\right) .
\end{aligned}
$$

A forma implícita (3.11) também permite a obtenção mais suave da matriz hessiana:

$$
\begin{aligned}
\frac{\partial^{2} \Phi_{l}(\eta \mid \mathbf{Z})}{\partial \eta_{2} \partial \eta_{2}^{\top}}= & {\left[\begin{array}{cc}
\frac{\partial^{2}}{\partial \boldsymbol{\theta} \partial \boldsymbol{\theta}^{\top}} \Phi(\eta) & \frac{\partial^{2}}{\partial \boldsymbol{\theta} \partial \gamma^{\top}} \Phi(\eta) \\
\frac{\partial^{2}}{\partial \boldsymbol{\theta} \partial \gamma^{\top}} \Phi(\eta) & \frac{\partial^{2}}{\partial \gamma \partial \gamma^{\top}} \Phi(\eta)
\end{array}\right] } \\
= & {\left[\begin{array}{cccc}
\frac{\partial^{2}}{\partial \alpha_{t}^{2}} \Phi_{l}(\eta \mid \mathbf{Z}) & \frac{\partial^{2}}{\partial \alpha_{t} \partial \lambda_{t}} \Phi_{l}(\eta \mid \mathbf{Z}) & \frac{\partial^{2}}{\partial \alpha_{t} \partial \alpha_{c}} \Phi_{l}(\eta \mid \mathbf{Z}) & \frac{\partial^{2}}{\partial \alpha_{t} \partial \lambda_{c}} \Phi_{l}(\eta \mid \mathbf{Z}) \\
\frac{\partial^{2}}{\partial \lambda_{t} \partial \alpha_{t}} \Phi_{l}(\eta \mid \mathbf{Z}) & \frac{\partial^{2}}{\partial \lambda_{t}^{2}} \Phi_{l}(\eta \mid \mathbf{Z}) & \frac{\partial^{2}}{\partial \lambda_{t} \partial \alpha_{c}} \Phi_{l}(\eta \mid \mathbf{Z}) & \frac{\partial^{2}}{\partial \lambda_{t} \partial \lambda_{c}} \Phi_{l}(\eta \mid \mathbf{Z}) \\
\frac{\partial^{2}}{\partial \alpha_{c} \partial \alpha_{t}} \Phi_{l}(\eta \mid \mathbf{Z}) & \frac{\partial^{2}}{\partial \alpha_{c} \partial \lambda_{t}} \Phi_{l}(\eta \mid \mathbf{Z}) & \frac{\partial^{2}}{\partial \alpha_{c}^{2}} \Phi_{l}(\eta \mid \mathbf{Z}) & \frac{\partial^{2}}{\partial \alpha_{c} \partial \lambda_{c}} \Phi_{l}(\eta \mid \mathbf{Z}) \\
\frac{\partial^{2}}{\partial \lambda_{c} \partial \alpha_{t}} \Phi_{l}(\eta \mid \mathbf{Z}) & \frac{\partial^{2}}{\partial \lambda_{c} \partial \lambda_{t}} \Phi_{l}(\eta \mid \mathbf{Z}) & \frac{\partial^{2}}{\partial \lambda_{c} \partial \alpha_{c}} \Phi_{l}(\eta \mid \mathbf{Z}) & \frac{\partial^{2}}{\partial \lambda_{c}^{2}} \Phi_{l}(\eta \mid \mathbf{Z})
\end{array}\right]_{4 \times 4} }
\end{aligned}
$$

que está na matriz hessiana resultante

$$
S=\left[\begin{array}{cccc}
\frac{\partial^{2}}{\partial \beta \partial \beta^{\top}} \Phi(\eta) & \frac{\partial^{2}}{\partial \beta \partial \phi^{\top}} \Phi(\eta) & \frac{\partial^{2}}{\partial \beta \partial \theta^{\top}} \Phi(\eta) & \frac{\partial^{2}}{\partial \beta \partial \gamma^{\top}} \Phi(\eta) \\
\frac{\partial^{2}}{\partial \phi \partial^{\top} \beta^{\top}} \Phi(\eta) & \frac{\partial^{2}}{\partial \phi \partial \phi^{\top}} \Phi(\eta) & \frac{\partial^{2}}{\partial \phi \partial \boldsymbol{\theta}^{\top}} \Phi(\eta) & \frac{\partial^{2}}{\partial \phi \partial \gamma^{\top}} \Phi(\eta) \\
\frac{\partial^{2}}{\partial \boldsymbol{\theta} \partial \boldsymbol{\beta}^{\top}} \Phi(\eta) & \frac{\partial^{2}}{\partial \boldsymbol{\theta} \partial \phi^{\top}} \Phi(\eta) & \frac{\partial^{2}}{\partial \boldsymbol{\theta} \partial \boldsymbol{\theta}^{\top}} \Phi(\eta) & \frac{\partial^{2}}{\partial \boldsymbol{\theta} \partial \gamma^{\top}} \Phi(\eta) \\
\frac{\partial^{2}}{\partial \gamma \partial \beta^{\top}} \Phi(\eta) & \frac{\partial^{2}}{\partial \gamma \partial \phi^{\top}} \Phi(\eta) & \frac{\partial^{2}}{\partial \gamma \partial \boldsymbol{\theta}^{\top}} \Phi(\eta) & \frac{\partial^{2}}{\partial \gamma \partial \gamma^{\top}} \Phi(\eta)
\end{array}\right]
$$

sendo, somente aqui, $\boldsymbol{\theta}=\left(\alpha_{t}, \lambda_{t}\right)^{\top}$ e $\gamma=\left(\alpha_{c}, \lambda_{c}\right)^{\top}$.

Devido a matriz hessiana 3.12 ter componentes com expressões demasiadamente extensas, foram incluídas todas aquelas expressões e suas demonstrações disponíveis somente no 
Apêndice B. Os resultados do gradiente e hessiana terão uma maior importância no texto quando a leitura estiver no Capítulo 4.

Para o modelo dessa seção, não desenvolvemos um estudo teórico para provar propriedades assintóticas. Entretanto, como é um modelo paramétrico, propriedades assintóticas do estimador de máxima verossimilhança devem ser válidas — porém ainda é preciso que sejam formalmente provadas.

\subsection{Modelo Semiparamétrico I}

Para essa segunda abordagem, aqui é apresentado e discutido o modelo semiparamétrico de taxas de falha proporcionais proposto por Xu et al. (2018) bem como um acréscimo a respeito de como lidar com as estimativas das funções basais e basais acumuladas. A seção é dividida em 4 subseções, sendo que as duas primeiras são destinadas a explanação da abordagem de aproximação das funções basais via método piecewise e o método via $M$-splines que é o acréscimo deste texto referente à contribuição original.

É importante ressaltar que o modelo proposto por Xu et al. (2018) é semelhante ao modelo paramétrico da seção anterior. A diferença é que agora a abordagem é semiparamétrica, sem a especificação da distribuição de $T$ e $C$. Assim o modelo é dado por

$$
\begin{aligned}
& \lambda_{T}(t \mid \mathbf{Z})=\lambda_{0 T}(x) \mathrm{e}^{\mathbf{Z}^{\top} \boldsymbol{\beta}}, \\
& \lambda_{C}(c \mid \mathbf{Z})=\lambda_{0 C}(x) \mathrm{e}^{\mathbf{Z}^{\top} \boldsymbol{\phi}},
\end{aligned}
$$

$\operatorname{com} \boldsymbol{\beta}=\boldsymbol{\beta}_{p \times 1}$ e $\phi=\phi_{p \times 1}$ vetores dos coeficientes de regressão para as funções de taxa associadas ao tempo de falha $T$ e ao tempo de censura $C$, respectivamente, e as funções não negativas $\lambda_{0 T}(t)$ e $\lambda_{0 C}(c)$ são as funções basais - funções que agora não são especificadas.

A seguir, são apresentadas duas abordagens para lidar com a não especificação das funções basais. Xu et al. (2018) trabalhou com uma abordagem via piecewise. Nós também trabalhamos com essa abordagem mas com uma distinção a respeito da partição do conjunto dos tempos efetivamente observáveis. Ademais, estimulados pelo excelente texto de Emura e Chen (2018), também trabalhamos com uma abordagem via M-splines.

\subsubsection{Abordagem Piecewise}

Emura e Chen (2016), Xu et al. (2018) e Emura e Chen (2018) mencionaram a dificuldade na estimação das funções basais via métodos convencionais para maximização direta em modelos semiparamétricos. Esse problema é causado pelo mal condicionamento em (3.3), pois é um problema de estimação infinito dimensional a partir de apenas um número finito de observações. Para aquelas referências, uma abordagem para contornar esse problema de maximização seria adotar uma aproximação por um espaço finito dimensional. Por problema infinito dimensional, Luenberger (1997) disse que, "em certos problemas de otimização, soluções ótimas desconhecidas podem não ser um número ou mesmo um vetor, mas uma quantidade contínua". Esse é 
o caso para a estimação da função basal do tempo, uma das medidas que é de interesse para este trabalho.

A função de verossimilhança de Xu et al. (2018) é dada por (3.3) sendo que a distribuição conjunta do tempo de falha e tempo de censura é obtida por meio de uma cópula arquimediana. Para contornar a dificuldade de um problema de maximização infinito dimensional, $\mathrm{Xu}$ et al. (2018) utilizaram a redução de espaço para um problema finito dimensional aplicando uma aproximação linear às funções basais dadas por

$$
\left\{\begin{array}{l}
\lambda_{0 T}(x)=\sum_{u=1}^{m} \theta_{u} \psi_{u}(x) \\
\lambda_{0 C}(x)=\sum_{u=1}^{m} \gamma_{u} \psi_{u}(x)
\end{array}\right.
$$

com $\psi_{1}, \psi_{2}, \ldots, \psi_{m}$ ditas funções de base não negativas. Para garantir que as funções basais sejam também funções não negativas, é, portanto, necessário restringir os parâmetros $\theta_{u} \geq 0 \mathrm{e}$ $\gamma_{u} \geq 0$ para $u=1,2, \ldots, m$. As respectivas funções basais acumuladas são

$$
\left\{\begin{array}{l}
\Lambda_{0 T}(x)=\sum_{u=1}^{m} \theta_{u} \Psi_{u}(x) \\
\Lambda_{0 C}(x)=\sum_{u=1}^{m} \gamma_{u} \Psi_{u}(x)
\end{array}\right.
$$

$\operatorname{com} \Psi_{u}(x)=\int_{0}^{x} \psi_{u}(w) d w$

Agora é rememorado como raciocínio a construção de uma partição de $m$ intervalos do conjunto total $\mathfrak{B}=\left[x_{(1)}, x_{(n)}\right]$ de tal modo que $\mathfrak{B}=\cup_{u=1}^{m} \mathfrak{B}_{u}$, para $\mathfrak{B}_{i} \cap \mathfrak{B}_{j}=\emptyset, \forall i \neq j$ e sendo $x_{(1)}$ e $x_{(n)}$ os respectivos mínimo e máximo da amostra de tempo efetivamente observável. Desse modo, a função de base é uma função indicadora tal qual $\psi_{u}(x)=I\left(x \in \mathfrak{B}_{u}\right)$, simplificando as igualdades anteriores por

$$
\begin{array}{ll}
\lambda_{0 T}(x)=\theta_{u} I\left(x \in \mathfrak{B}_{u}\right), & \Lambda_{0 T}(x)=\sum_{u=1}^{v} \theta_{u} \Psi_{u}(x), \text { se } x \in \mathfrak{B}_{v}, \\
\lambda_{0 C}(x)=\gamma_{u} I\left(x \in \mathfrak{B}_{u}\right), & \Lambda_{0 C}(x)=\sum_{u=1}^{v} \gamma_{u} \Psi_{u}(x), \operatorname{se} x \in \mathfrak{B}_{v},
\end{array}
$$

$\operatorname{com} \Psi_{u}(x)=s_{u}^{*}-l_{u}^{*}$ de $u=1,2, \ldots, v-1$ e $\Psi_{v}(x)=x-l_{u}^{*}$, em que $l_{u}^{*}$ e $s_{u}^{*}$ são os respectivos limites inferior e superior do intervalo $\mathfrak{B}_{u}$.

É aplicado uma adaptação na proposta de Xu et al. (2018) como segue

(P1) O conjunto total é definido como $\mathfrak{B}=\left[0, x_{(n)}\right]$; e

(P2) então, definimos duas partições. Uma partição $\mathfrak{I}=\left[0, t_{(n)}\right]$ para os tempos de falha e outra partição $\mathfrak{C}=\left[0, c_{(n)}\right]$ os tempos de censura dependente. Para $\mathfrak{I}$, tem-se $\mathfrak{I}=\cup_{u=1}^{m} \mathfrak{I}_{u}$, com $\mathfrak{I}_{i} \cap \mathfrak{I}_{j}=\emptyset, \forall i \neq j$ e sendo $t_{(n)}$ o máximo da amostra de tempos de falha efetivamente 
observáveis. Para $\mathfrak{C}$, tem-se $\mathfrak{C}=\cup_{u=1}^{m} \mathfrak{C}_{u}, \operatorname{com} \mathfrak{C}_{i} \cap \mathfrak{C}_{j}=\emptyset, \forall i \neq j$ e sendo $\mathfrak{c}_{(n)}$ o máximo da amostra de tempos de censura efetivamente observáveis.

Com as adaptações nos pontos (P1) e (P2), tem-se

$$
\left\{\begin{array}{l}
\lambda_{0 T}(x)=\theta_{u} \psi_{u}(x) \\
\lambda_{0 C}(x)=\gamma_{u} \omega_{u}(x)
\end{array}\right.
$$

$\operatorname{com} \psi_{u}(x)=I\left(x \in \mathfrak{I}_{u}\right)$ e $\omega_{u}(x)=I\left(x \in \mathfrak{C}_{u}\right)$. Agora as funções acumuladas são

$$
\left\{\begin{array}{l}
\Lambda_{0 T}(x)=\sum_{u=1}^{v} \theta_{u} \Psi_{u}(x), \text { se } x \in \mathfrak{T}_{v} \\
\Lambda_{0 C}(x)=\sum_{u=1}^{v} \gamma_{u} \Omega_{u}(x), \text { se } x \in \mathfrak{C}_{v}
\end{array}\right.
$$

$\operatorname{com} \Psi_{u}(x)=s_{u}^{*}-l_{u}^{*}$ de $u=1,2, \ldots, v-1$ e $\Psi_{v}(x)=x-l_{u}^{*}$, sendo $l_{u}^{*}$ e $s_{u}^{*}$ os respectivos limites inferior e superior do intervalo $\mathfrak{I}_{u}$. Analogamente, $\Omega_{u}(x)=s_{u}^{*}-l_{u}^{*}$ de $u=1,2, \ldots, v-1 \mathrm{e}$ $\Omega_{v}(x)=x-l_{u}^{*}$, sendo $l_{u}^{*}$ e $s_{u}^{*}$ os respectivos limites inferior e superior do intervalo $\mathfrak{C}_{u}$.

A construção da função de log-verossimilhança para o modelo semiparamétrico é exatamente como foi feita para o modelo paramétrico, excetuando que agora serão usadas outras abordagens para lidar com as funções basais. Portanto, a função de log-verossimilhança geral resultante para (3.4) pode ser escrita como

$$
\Phi_{l}(\eta \mid x, \mathbf{Z})=\sum_{i=1}^{n} \delta_{i T} l_{i T}+\delta_{i C} l_{i C}+\left(1-\delta_{i T}-\delta_{i C}\right) l_{i A}
$$

mas agora com suas componentes escritas

$$
\left\{\begin{array}{l}
l_{i T}=\log \boldsymbol{\psi}_{i}^{\top} \boldsymbol{\theta}+\mathbf{Z}_{i}^{\top} \boldsymbol{\beta}-\boldsymbol{\Psi}_{i}^{\top} \boldsymbol{\theta} \mathrm{e}^{\mathbf{Z}_{i}^{\top} \boldsymbol{\beta}}+\log K_{1}\left(\exp \left\{-\Psi_{i}^{\top} \boldsymbol{\theta} \mathrm{e}_{i}^{\mathbf{Z}_{i}^{\top} \boldsymbol{\beta}}\right\}, \exp \left\{-\boldsymbol{\Omega}_{i}^{\top} \boldsymbol{\gamma} \mathrm{e}_{i}^{\mathbf{Z}_{i}^{\top} \boldsymbol{\phi}}\right\}\right) \\
l_{i C}=\log \boldsymbol{\psi}_{i}^{\top} \boldsymbol{\gamma}+\mathbf{Z}_{i}^{\top} \boldsymbol{\phi}-\boldsymbol{\Psi}_{i}^{\top} \boldsymbol{\gamma} \mathrm{e}_{i}^{\top} \boldsymbol{\phi}+\log K_{2}\left(\exp \left\{-\Psi_{i}^{\top} \boldsymbol{\theta} \mathrm{e}_{i}^{\mathbf{Z}_{i}^{\top} \boldsymbol{\beta}}\right\}, \exp \left\{-\boldsymbol{\Omega}_{i}^{\top} \boldsymbol{\gamma} \mathrm{e}_{i}^{\top} \boldsymbol{\phi}\right\}\right) \\
l_{i A}=\log K\left(\exp \left\{-\Psi_{i}^{\top} \boldsymbol{\theta} \mathrm{e}_{i}^{\top} \boldsymbol{\beta}\right\}, \exp \left\{-\boldsymbol{\Omega}_{i}^{\top} \boldsymbol{\gamma} \mathrm{e}_{i}^{\top} \boldsymbol{\phi}\right\}\right)
\end{array}\right.
$$

sendo que

$$
\Psi_{i}=\left(\Psi_{1}\left(x_{i}\right), \Psi_{2}\left(x_{i}\right), \ldots, \Psi_{v}\left(x_{i}\right), 0, \ldots, 0\right)^{\top}
$$

para $x_{i} \in \mathfrak{I}_{v} \mathrm{e}$, analogamente,

$$
\boldsymbol{\Omega}_{i}=\left(\Omega_{1}\left(x_{i}\right), \Omega_{2}\left(x_{i}\right), \ldots, \Omega_{v}\left(x_{i}\right), 0, \ldots, 0\right)^{\top}
$$

para $x_{i} \in \mathfrak{V}_{v}$. Todas ferramentas para construção da aproximação das funções basais e basais acumuladas por partes estão escritas no Apêndice $B$, assim como todas as derivadas de primeira e segunda ordem com relação aos coeficientes $\theta_{u}$ e $\gamma_{u}$ para o resultante (3.13).

\subsubsection{Abordagem M-splines}

Xu et al. (2018) destacaram que as funções de base também podem ser funções M-splines ou por Kernel, mas preferiram não desenvolver além da abordagem via piecewise. Então, 
inspirados pelo trabalho de Emura e Chen (2018), adicionamos em (3.13) uma abordagem de aproximação para as funções basais e basais acumuladas via $M$-splines. Entretanto, antes de apresentar detalhadamente aquela abordagem, vale destacar o que seriam splines.

Para Ramsay et al. (tradução do autor, 1988), “o conceito de regressão polinomial utilizando splines em um intervalo definido $[L, U]$ é introduzido como polinômio por partes com restrições de continuidade bem especificadas". Vamos também utilizar as funções de base M-splines cúbicas assim como proposta por Ramsay et al. (1988) que utilizaram cinco parâmetros de aproximação que resultam em uma maior flexibilidade na estimação para aplicações reais. Para (3.13), foram inseridas $M$-splines nas funções de base gerais às basais

$$
\left\{\begin{array}{l}
\lambda_{0 T}\left(x_{i}\right)=\boldsymbol{M}_{i}^{\top} \boldsymbol{\theta}=\sum_{l=1}^{5} \theta_{l} M_{l}\left(x_{i}\right) \\
\lambda_{0 C}\left(x_{i}\right)=\boldsymbol{M}_{i}^{\top} \boldsymbol{\gamma}=\sum_{l=1}^{5} \gamma_{l} M_{l}\left(x_{i}\right)
\end{array}\right.
$$

sendo as basais acumuladas

$$
\left\{\begin{array}{l}
\Lambda_{0 T}\left(x_{i}\right)=\boldsymbol{I}_{i}^{\top} \boldsymbol{\theta}=\sum_{l=1}^{5} \theta_{l} I_{l}\left(x_{i}\right) \\
\Lambda_{0 C}\left(x_{i}\right)=\boldsymbol{I}_{i}^{\top} \boldsymbol{\gamma}=\sum_{l=1}^{5} \gamma_{l} I_{l}\left(x_{i}\right)
\end{array}\right.
$$

em que a função $I_{l}\left(x_{i}\right):=\int_{0}^{x_{i}} M_{l}(w) d w$ é denominada $I$-splines que está disponível no Apêndice B. Finalmente, as componentes da função de log-verossimilhança via aproximação $M$-splines são

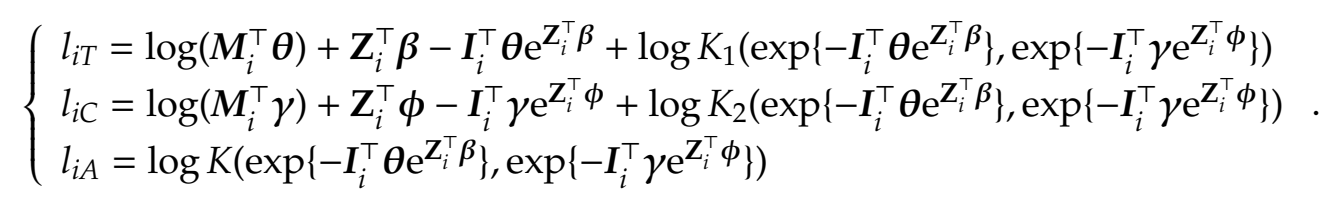

Assim como em Emura e Chen (2018), para calcular as funções de base $M_{l}$ é necessário especificar o comprimento do tempo $\left\{x_{1}, x_{2}, \ldots, x_{n}\right\}$. Foi adotada uma das possíveis escolhas por Emura e Chen (2018), reescrevendo o menor tempo observado como $\xi_{1}=x_{(1)}$ e o maior tempo observado como $\xi_{3}=x_{(n)}$. Assim, construiu-se três nós $\xi_{1}<\xi_{2}<\xi_{3}$ equidistantes com $\xi_{2}=\frac{\xi_{1}+\xi_{3}}{2}$. Ramsay et al. (1988) relataram que a abordagem por splines para aquelas restrições pode fornecer monotonicidade que é uma, entre outras, importante propriedade para muitos problemas de estimação de curvas. Outro fato importante a ressaltar é a facilidade para calcular as funções basais acumuladas usando aproximação via M-splines. Tal conveniência implica diretamente no ganho de velocidade computacional necessária para um posterior cálculo das estimativas.

Todos os cálculos de construção para os $M$-splines, $M_{l}$, assim como os $I$-splines, $I_{l}$, estão disponíveis no Apêndice B. Para o mesmo apêndice, também são fornecidas as derivadas de 
segunda ordem para essas funções. Aqui fica indicado que Emura e Chen (2018) também forneceram uma rotina livre no software R para calcular os M-splines e I-splines.

\subsubsection{Penalidade e Suavização}

Após mostrar o processo de introdução ao modelo semiparamétrico, os autores $\mathrm{Xu}$ et al. (2018) e Emura e Chen (2018) propuseram uma penalização na função de log-verossimilhança para estimação dos parâmetros. O intuito em inserir penalidade está na necessidade de suavizar as aproximações das funções basais discutidas nas subseções 3.4.1 e 3.4.2. É compreensível uma vez que, por exemplo, no caso piecewise podemos ter uma situação para gerar $m_{1}=30$ intervalos para a partição relacionada os tempos de falha e também mais $m_{2}=30$.

Como em Xu et al. (2017) e em Xu et al. (2018), este trabalho está interessado na estimação dos coeficientes de regressão que, por sua vez, depende das estimativas das quantidades contínuas $\lambda_{0 T}(t)$ e $\lambda_{0 C}(c)$. Para aquelas quantidades, foi aplicado uma aproximação linear tendo a inclusão dos parâmetros desconhecidos $\boldsymbol{\theta}$ e $\gamma$. Para cada um daqueles vetores de parâmetros desconhecidos, foi aplicada uma penalidade na função (3.13) e, desse modo, a função de máxima log-verossimilhança penalizada (MPL) é escrita como

$$
\Phi_{M P L}(\eta \mid x, \mathbf{Z})=\Phi_{l}(\eta \mid x, \mathbf{Z})-h_{1} J_{1}(\theta)-h_{2} J_{2}(\gamma),
$$

sendo $J_{1}(\theta)$ e $J_{2}(\gamma)$ funções de suavidade com seus respectivos coeficientes de suavidade $h_{1} \geq 0$ e $h_{2} \geq 0$. Assim como dito em Xu et al. (2017), existem escolhas comuns para aquelas funções de suavidade como $\sum_{v=1}^{m}\left(\theta_{v}-\theta_{v-1}\right)^{2}$ ou $\sum_{v=1}^{m}\left(\theta_{v+1}-2 \theta_{v}+\theta_{v-1}\right)^{2}$, com $\theta_{0}=\theta_{1}$ e $\theta_{m+1}=\theta_{m}$. Foi adotada a primeira opção e ela foi reescrita por conveniência na forma quadrática $J_{1}(\boldsymbol{\theta})=\boldsymbol{\theta}^{\top} \mathbf{R}_{1} \boldsymbol{\theta}$ e $J_{2}(\gamma)=\gamma^{\top} \mathbf{R}_{2} \gamma$, em que $\mathbf{R}_{1}$ e $\mathbf{R}_{2}$ são matrizes tridiagonais que estão disponíveis no Apêndice B. No caso das funções de base via M-splines, Emura e Chen (2018) previamente reescreveram as funções de suavidade convenientemente na forma quadrática como as matrizes $\mathbf{R}_{1}$ e $\mathbf{R}_{2}$ também disponíveis no Apêndice B.

Por mais que possa inicialmente fixar tanto o número de intervalos $m_{1}$ e $m_{2}$ e as quantidades de penalização $h_{1}$ e $h_{2}$, o descuido poderá facilmente levar-nos a situações inflexíveis. James et al. (2013) também nomearam os coeficientes $h_{1}$ e $h_{2}$ como coeficientes de perturbação uma vez que é natural que, por exemplo, a escolha de um número pequeno de intervalos ou um número grande de suavização produzirá uma estimativa ultra suavizada com pequena variância mas com grande viés. Quando é imposta a situação contrária quanto ao número de intervalos e parâmetros de perturbação, deparamo-nos com uma estimativa com pequeno viés mas de elevada variância. Um bom texto que discute o trade-off entre variância e viés foi escrito por Friedman et al. (2001). Assim como foi em Xu et al. (2017) e Xu et al. (2018), optou-se pela combinação das abordagens de optimização daquelas quantidades usando o critério de Akaike (1974) para o número de intervalos e o critério de O'Sullivan (1988) para definir as quantidades de perturbação $h_{1}$ e $h_{2}$.

Primeiramente, também como descrito em Xu et al. (2017), são fixados os parâmetros de 
perturbação como $h_{1}=h_{2}=0$ e logo em seguida são selecionados

$$
\hat{m}=\underset{m}{\arg \min }\left\{\operatorname{AIC}(m)=2 m-\Phi_{l}(\hat{\eta} \mid x, \mathbf{Z})\right\},
$$

tal que $\hat{\eta}$ é o vetor de estimativas de máxima verossimilhança do vetor $\eta$ do modelo resultante (3.13). Na abordagem de não utilizar a função MPL, apenas aplicaríamos a seleção do $\hat{m}$ como exemplificado em Shih e Emura (2018). Todavia, vamos trabalhar com a abordagem descrita em Emura e Chen (2018) que, sob a premissa de independência $K(a, b)=a b$, a função MPL é reescrita como a soma de duas funções MPL marginais dadas por

$$
\left\{\begin{array}{l}
\Phi_{M P L}^{[1]}(\boldsymbol{\beta}, \boldsymbol{\theta} \mid \boldsymbol{x}, \mathbf{Z})=\sum_{i=1}^{n} \log \lambda_{0 T}\left(x_{i}\right)+\mathbf{Z}_{i}^{\top} \boldsymbol{\beta}-3 \Lambda_{T}\left(x_{i}\right)-h_{1} \boldsymbol{J}_{1} \\
\Phi_{M P L}^{[2]}(\boldsymbol{\phi}, \boldsymbol{\gamma} \mid \boldsymbol{x}, \mathbf{Z})=\sum_{i=1}^{n} \log \lambda_{0 C}\left(x_{i}\right)+\mathbf{Z}_{i}^{\top} \boldsymbol{\phi}-3 \Lambda_{C}\left(x_{i}\right)-h_{2} \boldsymbol{J}_{2}
\end{array} .\right.
$$

Eles sugeriram selecionar os parâmetros de perturbação $h_{1}$ e $h_{2}$ baseando-se no método de validação cruzada que minimiza

$$
L C V_{1}=\Phi_{M P L}^{[1]}(\hat{\boldsymbol{\beta}}, \hat{\boldsymbol{\theta}} \mid \boldsymbol{x}, \mathbf{Z})-\operatorname{tr}\left\{\hat{\boldsymbol{H}}_{P L 1}^{-1} \hat{\boldsymbol{H}}_{1}\right\} \quad \text { e } \quad L C V_{2}=\Phi_{M P L}^{[2]}(\hat{\boldsymbol{\phi}}, \hat{\boldsymbol{\gamma}} \mid \boldsymbol{x}, \mathbf{Z})-\operatorname{tr}\left\{\hat{\boldsymbol{H}}_{P L 2}^{-1} \hat{\boldsymbol{H}}_{2}\right\},
$$

sendo $\hat{\boldsymbol{\beta}}, \hat{\boldsymbol{\theta}}, \hat{\boldsymbol{\phi}}$ e $\hat{\boldsymbol{\gamma}}$ as estimativas das funções MPL marginais a cima, $\hat{\boldsymbol{H}}_{P L 1}^{-1}$ e $\hat{\boldsymbol{H}}_{P L 2}^{-1}$ são as inversas das matrizes hessianas para as estimativas da respectiva função MPL resultante (3.14). Além disso, $\operatorname{tr}(\boldsymbol{A})$ denota o traço da matriz $\boldsymbol{A}, \hat{\boldsymbol{H}}_{1}$ e $\hat{\boldsymbol{H}}_{2}$ são as matrizes hessianas para as estimativas da função resultante 3.13 dadas por

$$
\hat{\boldsymbol{H}}_{1}=\hat{\boldsymbol{H}}_{P L 1}+2 h_{1}\left[\begin{array}{cc}
\boldsymbol{O}_{p \times p} & \boldsymbol{O}_{p \times m_{1}} \\
\boldsymbol{O}_{m_{1} \times p} & \boldsymbol{R}_{m_{1} \times m_{1}}
\end{array}\right] \text { e } \quad \hat{\boldsymbol{H}}_{2}=\hat{\boldsymbol{H}}_{P L 2}+2 h_{2}\left[\begin{array}{cc}
\boldsymbol{O}_{p \times p} & \boldsymbol{O}_{p \times m_{2}} \\
\boldsymbol{O}_{m_{2} \times p} & \boldsymbol{R}_{m_{2} \times m_{2}}
\end{array}\right],
$$

em que $\boldsymbol{O}$ é matriz nula. Destacamos que Emura e Chen (2018) disponibilizaram um pacote no software R, mas sob um modelo diferente do qual estamos trabalhando.

\subsubsection{Problema de Otimização com Restrições}

É notório que a literatura seja repleta de informação sobre a dificuldade em resolver um problema de otimização com restrições. Entretanto, antes de adentrar mais nos pontos de dificuldade para o problema em questão, serão explanadas algumas conveniências em notação a seguir. Primeiramente, será particionado o vetor de parâmetros dos coeficientes de regressão pela igualdade $\eta=\left(\eta_{1}, \eta_{2}\right)^{\top}$, nos quais $\eta_{1}=(\beta, \phi)^{\top}$ e $\eta_{2}=(\theta, \gamma)^{\top}$. Então o objetivo aqui é atender

$$
\left(\hat{\eta}_{1}, \hat{\eta}_{2}\right)=\underset{\eta_{2} \geq 0}{\arg \max } \Phi_{M P L}\left(\eta_{1}, \eta_{2} \mid x, \mathbf{Z}\right)
$$

A restrição $\eta_{2} \geq 0$ torna-se um empecilho quando se pensa em utilizar métodos convencionais para soluções de problemas de otimização para funções não lineares. Como a função objetivo resultante (3.14) pode possuir vários máximos locais, a dificuldade aumenta. O custo computacional é a pior consequência. Abordagens como em Bertsekas (2014) aplicando o 
método de Lagrange-Newton para encontrar pontos de mínimos (máximos) locais com uma função de penalidade requerem um considerável esforço computacional para o modelo resultante proposto por Xu et al. (2018). Xu et al. (2017) relataram que o tempo de convergência é dez a vinte vezes mais custoso.

Ma (2010) propôs um algoritmo Multiplicativo Iterativo (MI) para reconstrução de imagem de tomografia. A função objetivo é como uma função resultante (3.14). Os pontos positivos para essa abordagem é o ganho computacional em velocidade do algoritmo quanto a convergência. A próxima etapa desse texto é explicar como Xu et al. (2018) adaptaram esse algoritmo.

\subsubsection{Método Iterativo-Multiplicativo}

Chegamos na subseção mais sofisticada do algoritmo que usamos para obter os resultados no Capítulo 4. Para motivar o leitor, questione-se sobre a dificuldade computacional em ajustar um conjunto de dados efetivamente observáveis que exija uma partição de, por exemplo, $m_{1}=m_{2}=30$ intervalos. Adicionalmente, questione-se adicionando ainda com um estudo de simulação com tamanho $N=1000$.

Para um método computacional por iterações, geralmente é necessário obter o vetor gradiente e a matriz hessiana. Então temos um vetor de comprimento $p+60$ e matriz hessiana de ordem $(p+60) \times(p+60)$ para o caso da abordagem via piecewise. A princípio, o usuário do algoritmo ficará computacionalmente dependente do poder de sua máquina. Então é aqui que entra a proposta usada por Xu et al. (2018), originalmente implementada por Ma (2010) e Ma et al. (2014): uma abordagem sutil que permite uma junção de métodos de iteração de tal forma que podemos particionar o vetor $\eta=\left(\eta_{1}, \eta_{2}\right)^{\top}$, melhorando o desempenho computacional.

A condição necessária de Karush-Kuhn-Tucker (veja Luenberger, 1997,página 247) para obter (3.15) é

$$
\begin{gathered}
\frac{\partial \Phi_{M P L}}{\partial \beta_{j}}=0 \text { e } \frac{\partial \Phi_{M P L}}{\partial \phi_{j}}=0 \\
\frac{\partial \Phi_{M P L}}{\partial \theta_{u}}=0 \quad \text { se } \theta_{u}>0 \quad \text { e } \frac{\partial \Phi_{M P L}}{\partial \theta_{u}}<0 \quad \text { se } \theta_{u}=0, \\
\frac{\partial \Phi_{M P L}}{\partial \gamma_{u}}=0 \quad \text { se } \gamma_{u}>0 \quad \text { e } \frac{\partial \Phi_{M P L}}{\partial \gamma_{u}}<0 \quad \text { se } \gamma_{u}=0,
\end{gathered}
$$

para $j=1,2, \ldots, p$ e $u=1,2, \ldots, m$ (para o caso de $m=m_{1}=m_{2}$ ).

Assim como em Ma et al. (2014), a condição necessária para obter (3.15) é resolver as igualdades

$$
\begin{aligned}
& \theta_{u}\left(\sum_{i=1}^{n} \frac{\partial \Phi_{l}}{\partial \theta_{u}}-h_{1} \frac{\partial J_{1}}{\partial \theta_{u}}\right)=0, \\
& \gamma_{u}\left(\sum_{i=1}^{n} \frac{\partial \Phi_{l}}{\partial \gamma_{u}}-h_{2} \frac{\partial J_{2}}{\partial \gamma_{u}}\right)=0 .
\end{aligned}
$$

Segundo Ma (2010), a chave para desenvolver o algoritmo MI é reescrever o lado esquerdo 
das últimas igualdades por uma manipulação matemática simples tais quais

$$
\begin{aligned}
& \theta_{u}\left(-\sum_{i=1}^{n}\left[\frac{\partial \Phi_{l}}{\partial \theta_{u}}\right]^{+}+\left[\frac{\partial \Phi_{l}}{\partial \theta_{u}}\right]^{-}+h_{1}\left[\frac{\partial J_{1}}{\partial \theta_{u}}\right]^{+}\right)=\theta_{u}\left(\sum_{i=1}^{n}\left[\frac{\partial \Phi_{l}}{\partial \theta_{u}}\right]^{+}+\left[\frac{\partial \Phi_{l}}{\partial \theta_{u}}\right]^{-}-h_{1}\left[\frac{\partial J_{1}}{\partial \theta_{u}}\right]^{-}\right), \\
& \gamma_{u}\left(-\sum_{i=1}^{n}\left[\frac{\partial \Phi_{l}}{\partial \gamma_{u}}\right]^{+}+\left[\frac{\partial \Phi_{l}}{\partial \gamma_{u}}\right]^{-}+h_{2}\left[\frac{\partial J_{2}}{\partial \gamma_{u}}\right]^{+}\right)=\gamma_{u}\left(\sum_{i=1}^{n}\left[\frac{\partial \Phi_{l}}{\partial \gamma_{u}}\right]^{+}+\left[\frac{\partial \Phi_{l}}{\partial \gamma_{u}}\right]^{-}-h_{2}\left[\frac{\partial J_{2}}{\partial \gamma_{u}}\right]^{-}\right),
\end{aligned}
$$

sendo $z=[z]^{+}+[z]^{-}$em que $[z]^{+}=\max (0, z)$ é a componente positiva e $[z]^{-}=\min (0, z)$ componente negativa. Então expressaram o algoritmo

$$
\theta_{u}^{\left(k+\frac{1}{2}\right)}=\theta_{u}^{(k)} \frac{\xi_{u 11}^{(k)}}{\xi_{u 21}^{(k)}} \quad \text { e } \gamma_{u}^{\left(k+\frac{1}{2}\right)}=\gamma_{u}^{(k)} \frac{\xi_{u 21}^{(k)}}{\xi_{u 22}^{(k)}}
$$

nos quais

$$
\begin{aligned}
& \xi_{u 12}=\left(-\sum_{i=1}^{n}\left[\frac{\partial \Phi_{l}}{\partial \theta_{u}}\right]^{+}+\left[\frac{\partial \Phi_{l}}{\partial \theta_{u}}\right]^{-}+h_{1}\left[\frac{\partial J_{1}}{\partial \theta_{u}}\right]^{+}\right) \text {e } \quad \xi_{u 11}=\left(\sum_{i=1}^{n}\left[\frac{\partial \Phi_{l}}{\partial \theta_{u}}\right]^{+}+\left[\frac{\partial \Phi_{l}}{\partial \theta_{u}}\right]^{-}-h_{1}\left[\frac{\partial J_{1}}{\partial \theta_{u}}\right]^{-}\right), \\
& \xi_{u 22}=\left(-\sum_{i=1}^{n}\left[\frac{\partial \Phi_{l}}{\partial \gamma_{u}}\right]^{+}+\left[\frac{\partial \Phi_{l}}{\partial \gamma_{u}}\right]^{-}+h_{1}\left[\frac{\partial J_{1}}{\partial \gamma_{u}}\right]^{+}\right) \text {e } \quad \xi_{u 21}=\left(\sum_{i=1}^{n}\left[\frac{\partial \Phi_{l}}{\partial \gamma_{u}}\right]^{+}+\left[\frac{\partial \Phi_{l}}{\partial \gamma_{u}}\right]^{-}-h_{1}\left[\frac{\partial J_{1}}{\partial \gamma_{u}}\right]^{-}\right),
\end{aligned}
$$

resultando no algoritmo do gradiente

$$
\theta_{u}^{\left(k+\frac{1}{2}\right)}=\theta_{u}^{(k)}+\frac{\theta_{u}^{(k)}}{\xi_{u 12}} \nabla \Phi_{M P L}^{(k)} \quad \text { e } \quad \gamma_{u}^{\left(k+\frac{1}{2}\right)}=\gamma_{u}^{(k)}+\frac{\gamma_{u}^{(k)}}{\xi_{u 22}} \nabla \Phi_{M P L^{\prime}}^{(k)}
$$

$\operatorname{com} \nabla \Phi_{M P L}^{(k)}$ o gradiente da função de log-verossimilhança penalizada. Então, finalmente, obter a $(k+1)$-ésima iteração

$$
\theta_{u}^{(k+1)}=\theta_{u}^{(k)}+\alpha^{(k)}\left(\theta_{u}^{\left(k+\frac{1}{2}\right)}-\theta_{u}^{(k)}\right) \quad \text { e } \gamma_{u}^{(k+1)}=\gamma_{u}^{(k)}+\alpha^{(k)}\left(\gamma_{u}^{\left(k+\frac{1}{2}\right)}-\gamma_{u}^{(k)}\right)
$$

sendo $\alpha \in[0,1]$ o chamado step-size ou step-length de uma linha de busca como da regras de Armijo e Wolfe, que serão brevemente discutidas mais adiante.

\section{Algoritmo Newton-MI}

Aqui é apresentado um algoritmo que funciona como uma adaptação do Newton-Raphson com o algoritmo Multiplicativo-Iterativo utilizado por Xu et al. (2018) para obter as estimativas dos coeficientes de regressão para os tempos de sobrevivência $T$ e censura $C$. Eles usaram um algoritmo semelhante utilizado por Ma et al. (2014).

Seja $\eta_{1}^{(k)}$ o valor do vetor de coeficientes de regressão $\eta_{1}$ na $k$-ésima iteração. Caso a matriz hessiana de $\Phi_{M P L}\left(\eta_{1}^{(k)}, \eta_{2}^{(k)}\right)$ for matriz negativa definida na próxima $(k+1)$-ésima iteração, então para obter a atualização $\eta_{1}^{(k+1)}$ será utilizado o algoritmo de Newton-Raphson. Caso contrário, será utilizado o algoritmo de Newton-Gauss. O esquema pode ser apresentado no único 
resultado

$$
\eta_{1}^{(k+1)}=\eta_{1}^{(k)}+\omega_{1}^{(k)} \mathbf{S}_{1} \nabla \Phi_{M P L}\left(\eta_{1}^{(k)}, \eta_{2}^{(k)}\right)
$$

com $\mathbf{S}_{1}$ a inversa da matriz hessiana negativa definida de $\Phi_{M P L}\left(\eta_{1}^{k}, \eta_{2}^{(k)}\right)$ ou a inversa de $\frac{\partial^{2}}{\partial \eta_{1} \partial \eta_{1}^{\top}} \Phi_{M P L}\left(\eta_{1}^{k}, \eta_{2}^{(k)}\right)$ caso for utilizado o algoritmo de Newton-Gauss. Na prática, quando o algoritmo está sendo utilizado para atualizar $\eta_{1}$ na $(k+1)$-ésima iteração, a matriz hessiana pode ficar refém da iteração anterior a respeito da propriedade de ser uma matriz negativadefinida. Portanto, utilizar o algoritmo de Newton-Gauss é uma saída para contornar esse problema até que a linha de busca seja atualizada por intermédio do step-size $\omega_{1}^{(k)} \in(0,1]$ que garante $\Phi_{M P L}\left(\eta_{1}^{(k+1)}, \eta_{2}^{(k)}\right) \geq \Phi_{M P L}\left(\eta_{1}^{(k)}, \eta_{2}^{(k)}\right)$. Outra dificuldade na prática é que o algoritmo de Newton-Gauss tem um custo computacional elevadíssimo quanto a convergência.

Agora, seja $\eta_{2}^{(k)}$ o valor do vetor de coeficientes de $\eta_{2}$ na $k$-ésima iteração. Para atualizar o vetor de coeficientes de aproximação das funções basais e basais acumuladas, o esquema de atualização é apresentado no único resultado

$$
\eta_{2}^{(k+1)}=\eta_{2}^{(k)}+\omega_{2}^{(k)} \mathbf{S}_{2} \nabla \Phi_{M P L}\left(\eta_{1}^{(k+1)}, \eta_{2}^{(k)}\right)
$$

com $\mathbf{S}_{2}$ uma matriz diagonal cujas componentes são $\left(\frac{\theta_{1}^{(k)}}{\xi_{11}^{(k)}}, \ldots, \frac{\theta_{n}^{(k)}}{\xi_{1 n}^{(k)}}, \frac{\gamma_{1}^{(k)}}{\xi_{21}}, \ldots, \frac{\gamma_{n}^{(k)}}{\xi_{2 n}^{(k)}}\right)$ que estão explicitadas no Apêndice B e $\omega_{2}^{(k)} \in(0,1]$ sendo o step-size que faz a linha de busca garantir que $\Phi_{M P L}\left(\boldsymbol{\eta}_{1}^{(k+1)}, \boldsymbol{\eta}_{2}^{(k+1)}\right) \geq \Phi_{M P L}\left(\boldsymbol{\eta}_{1}^{(k+1)}, \boldsymbol{\eta}_{2}^{(k)}\right)$. Todas as propriedades de convergência do algoritmo Newton-MI estão demonstradas e discutidas em Ma (2010) e Ma et al. (2014).

O Quadro 1 apresenta o algoritmo Newton-MI como um esboço ilustrativo para compreender melhor a explicação de como se deve usar o método MI para obter as estimativas. As variáveis de entrada para o algoritmo são um conjunto de dados sob censura dependente $\left\{i: x_{i}, \delta_{i T}, \delta_{i C}, \mathbf{Z}_{i}\right\}$, um valor inicial $\eta^{(1)}$ para o vetor $\boldsymbol{\eta}$ que esteja em uma região confortável $\mathfrak{F}$ que pode coincidir região de vizinhança do ponto de máximo $\hat{\eta}$ e $K_{\alpha}$ representa um membro da classe arquimediana com seu respectivo coeficiente de dependência $\alpha$ conhecido.

Sobre a região confortável $\mathfrak{F}$, determiná-la não é uma tarefa simples sob o aspecto computacional. Ela geralmente é um subconjunto próprio de $\left\{\eta_{1} \in R^{p}, \eta_{2} \geq 0\right\}$ que dependerá implicitamente do conjunto de dados disponível, sendo possível que para duas réplicas podese ter duas regiões $\mathfrak{F}_{1}$ e $\mathfrak{F}_{2}$ tal que $\mathfrak{F}_{1} \backslash \mathfrak{F}_{2} \neq \emptyset$. Felizmente, apesar dessa região $\mathfrak{F}$ ser difícil de determinar, é fácil obter um subconjunto que possa satisfazer perfeitamente para escolher um ponto (vetor) para iniciar o algoritmo no exercício de simulação do Capítulo 4. Outro desafio computacional é na obtenção da linha de busca por intermédio do step-size porque esta linha também é implicitamente dependente da região $\mathfrak{F}$. Existem alternativas e adaptações mas este texto usa apenas as abordagens de Armijo e Wolfe em Luenberger (1997).

As linhas de busca de Armijo e Wolfe funcionam como condições para obter uma direção restrita do vetor estimado no algoritmo MI. São regras necessárias e importantíssimas no algoritmo que optamos. No algoritmo MI, os step-length $\omega_{1}$ e $\omega_{2}$ satisfazem as condições de Armijo (Wolfe) se 


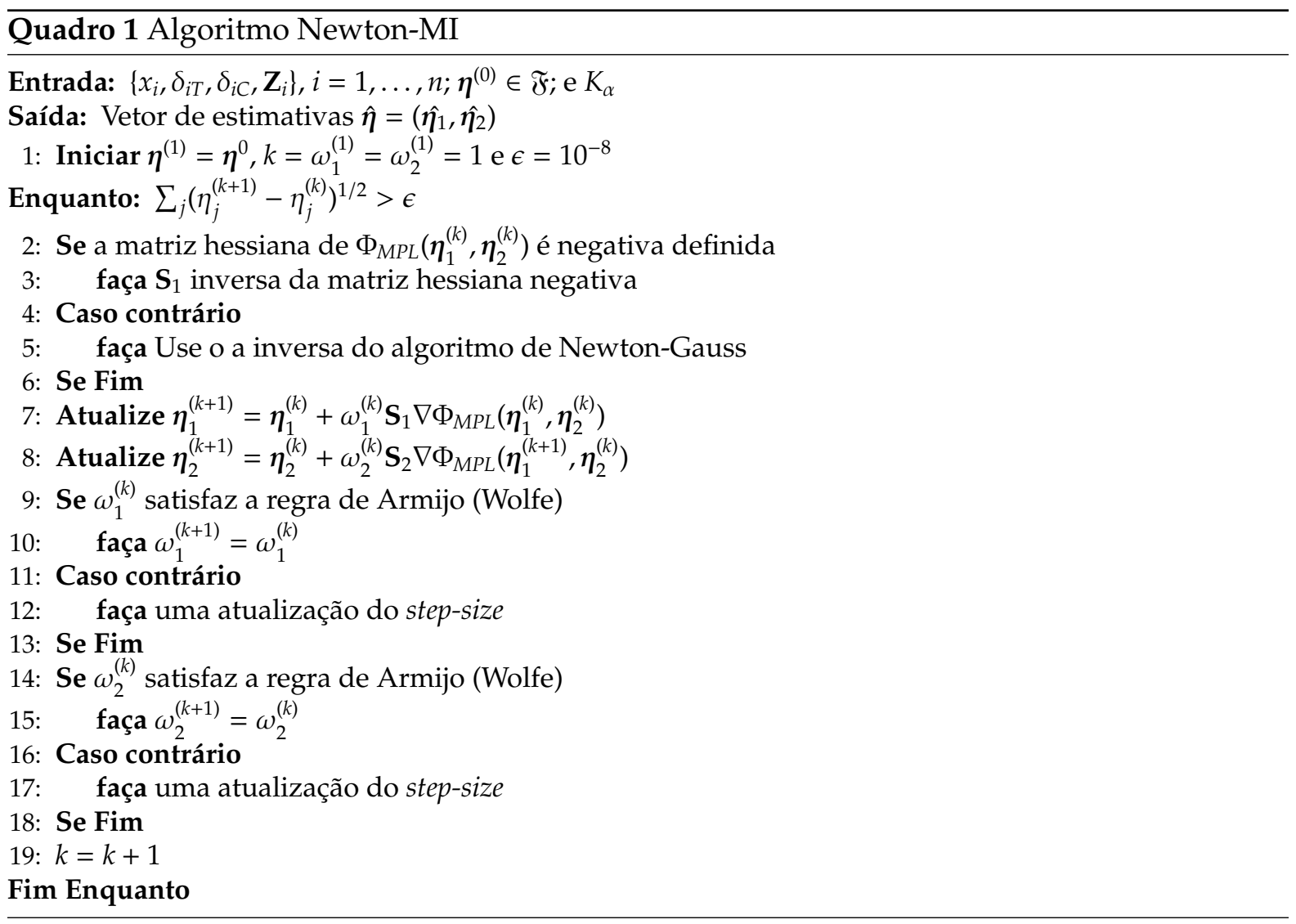

(C1)

$$
\begin{gathered}
\Phi_{M P L}\left(\boldsymbol{\eta}_{1}^{(k+1)}, \boldsymbol{\eta}_{2}^{(k)}\right) \leq \Phi_{M P L}\left(\boldsymbol{\eta}_{1}^{(k+1)}, \boldsymbol{\eta}_{2}^{(k)}\right)+c_{1} \omega_{1}^{(k)}\left[\boldsymbol{p}_{1}^{\top}\right]^{(k)} \frac{\partial}{\partial \boldsymbol{\eta}_{1}} \Phi_{M P L}\left(\boldsymbol{\eta}_{1}^{(k+1)}, \boldsymbol{\eta}_{2}^{(k)}\right), \\
-\left[\boldsymbol{p}_{1}^{\top}\right]^{(k)} \frac{\partial}{\partial \boldsymbol{\eta}_{1}} \Phi_{M P L}\left(\boldsymbol{\eta}_{1}^{(k+1)}, \boldsymbol{\eta}_{2}^{(k)}\right) \leq-c_{2} \frac{\partial}{\partial \boldsymbol{\eta}_{1}} \Phi_{M P L}\left(\boldsymbol{\eta}_{1}^{(k+1)}, \boldsymbol{\eta}_{2}^{(k)}\right) ;
\end{gathered}
$$

$$
\begin{gathered}
\Phi_{M P L}\left(\boldsymbol{\eta}^{(k+1)}\right) \leq \Phi_{M P L}\left(\boldsymbol{\eta}^{(k+1)}\right)+c_{3} \omega_{2}^{(k)}\left[\boldsymbol{p}_{2}^{\top}\right]^{(k)} \frac{\partial}{\partial \eta_{2}} \Phi_{M P L}\left(\boldsymbol{\eta}^{(k+1)}\right) ; \mathrm{e} \\
-\left[\boldsymbol{p}_{2}^{\top}\right]^{(k)} \frac{\partial}{\partial \eta_{2}} \Phi_{M P L}\left(\boldsymbol{\eta}^{(k+1)}\right) \leq-c_{4} \frac{\partial}{\partial \eta_{2}} \Phi_{M P L}\left(\boldsymbol{\eta}^{(k+1)}\right)
\end{gathered}
$$

sendo as constantes $0<c_{1}<c_{2}<1,0<c_{3}<c_{4}<1$, as componentes $\left[p_{1}^{\top}\right]^{(k)}$ e $\left[p_{2}^{\top}\right]^{(k)}$ são os produtos da matrizes hessiana negativa, $S_{1}$ e $S_{2}$, e os respectivos gradientes na $k$-ésima iteração descritos nas linhas 7 e 8 no Quadro 1 . Já os step-length $\omega_{1}$ e $\omega_{2}$ estão em $[0,1]$. Caso as condições (C1) ou (C2) não sejam satisfeitas, atualizamos os step-length multiplicando por uma constante, por exemplo, $\omega_{j}^{(k+1)}=0,6 \omega_{j}^{(k)}$ para $j=1,2$.

\subsubsection{Propriedades assintóticas}

Xu et al. (2018) provaram que seu modelo semiparamétrico possui duas propriedades ao estimador do parâmetro $\eta$ : (3.4.6.1) consistência; e (3.4.6.2) convergência à distribuição normal 
multivariada. Então essa subseção foi elaborada para discuti-las. As pressuposições são apresentadas a seguir:

(A1) os dados efetivamente observáveis $\left(X_{i}, \delta_{i T}, \delta_{i C}, \mathbf{Z}\right)$ são vetores aleatórios, independentes e identicamente distribuídos sendo a distribuição de $\mathbf{Z}_{i}$ independente de $\eta$;

(A2) existe um espaço paramétrico $\Theta$ para $\eta$ tal que $\Theta$ seja um subconjunto compacto em $\mathbb{R}^{2(p+m)}$. É necessário assumir que $\mathbb{E}_{\eta_{0}}\left(n^{-1} \Phi_{M P L}(\eta \mid x, Z)\right)$ exista e que $\eta_{0} \in \Theta$ seja seu único ponto de máximo; e

(A3) a função de log-verossimilhança, $\Phi_{M P L}(\eta \mid x, Z)$, é diferenciável até segunda ordem na vizinhança de $\eta_{0}$ e as matrizes

$$
\lim _{n \rightarrow \infty} \frac{\partial}{\partial \eta} \Phi_{M P L}(\eta \mid x, \mathbf{Z}) \frac{\partial}{\partial \eta^{\top}} \Phi_{M P L}(\eta \mid x, \mathbf{Z}) \quad \text { e } \quad \lim _{n \rightarrow \infty} \frac{\partial^{2}}{\partial \eta \partial \eta^{\top}} \Phi_{M P L}(\eta \mid x, \mathbf{Z})
$$

existem.

Originalmente, Xu et al. (2018) apresentaram cinco premissas. As duas últimas são:

(A4) as funções de penalização $J_{1}$ e $J_{2}$ são contínuas e limitadas sobre o espaço $\Theta$, assim como são funções diferenciáveis até segunda ordem, ademais, limitadas na vizinhança de $\eta_{0}$; e

(A5) assume-se que existam $q$ restrições para $\boldsymbol{\theta}$ e $r$ restrições para $\gamma$. Então seja $\mathbf{U}$ a matriz cujas linhas correspondem as restrições tomando valor igual a zero e as demais linhas formando uma matriz identidade. Agora seja

$$
\boldsymbol{F}_{0}(\boldsymbol{\eta})=-n^{-1} \mathbb{E}_{\eta_{0}} \frac{\partial^{2}}{\partial \eta \partial \eta^{\top}} \Phi_{M P L}(\eta \mid x, \mathbf{Z})+h_{1} \frac{\partial^{2}}{\partial \boldsymbol{\theta} \partial \boldsymbol{\theta}^{\top}} J_{1}(\boldsymbol{\theta})+h_{1} \frac{\partial^{2}}{\partial \gamma \partial \gamma^{\top}} J_{2}(\gamma)
$$

uma matriz tal que, na vizinhança de $\eta_{0}$, a matriz $\mathbf{U}^{\top} \boldsymbol{F}_{0}(\eta) \mathbf{U}$ seja invertível.

Como nossa abordagem no próximo Capítulo se dará utilizando uma partição pelos quantis, por ora, somente é necessário entender que não há restrições e a matriz $\mathbf{U}$ é uma matriz identidade. Portanto, utilizando todas as cinco últimas premissas a cima, pode-se provar que

$$
n^{1 / 2}\left(\hat{\boldsymbol{\eta}}-\boldsymbol{\eta}_{0}\right) \stackrel{D}{\rightarrow} N_{2(p+m)}\left(\mathbf{0}_{2(p+m)}, \boldsymbol{F}_{0}(\boldsymbol{\eta})^{-1} \boldsymbol{G}_{0}(\boldsymbol{\eta})\left[\boldsymbol{F}_{0}(\boldsymbol{\eta})^{-1}\right]^{\top}\right),
$$

sendo $G_{0}(\eta)=\mathbb{E}_{\eta_{0}} \frac{\partial^{2}}{\partial \eta \partial \eta^{\top}} \Phi_{M P L}(\eta \mid x, \mathbf{Z})$. Uma demonstração já foi disponibilizada pelos autores em Xu et al. (2018) e, portanto, decidimos não escrevê-la. Eles comentam que a matriz $\boldsymbol{F}_{0}(\boldsymbol{\eta})$ é de difícil expressão analítica e uma abordagem mais computacionalmente eficiente para obtê-la é assumindo que $G_{0}(\eta)$ seja uma aproximação da matriz hessiana negativa.

Já a propriedade da consistência, Xu et al. (2018) demonstram na parte suplementar do seu trabalho. Como é uma demonstração extensa, decidimos não apresenta-la aqui. Entretanto, faz-se necessário destacar suas principais pressuposições a seguir:

(A6) A matriz $\mathbf{Z}$ deve ser limitada e $\mathbb{E}\left(\mathbf{Z Z}^{\top}\right)$ é não-singular; 
(A7) as funções de penalidade $J_{1}$ e $J_{2}$ são limitadas;

(A8) para $\lambda_{0 T}(t)$ e $\lambda_{0 C}(c)$, seus correspondentes parâmetros $\theta_{m}$ e $\gamma_{m}$ estão em um subconjunto compacto do $\mathbb{R}^{m}$ como todos seus elementos são limitados;

(A9) assumindo que para qualquer $\lambda_{0 T}(t)$ e $\lambda_{0 C}(c)$, existem $\lambda_{n T}(t)$ e $\lambda_{n C}(c)$ tal que

$$
\max _{x}\left|\lambda_{n T}(t)-\lambda_{0 T}(t)\right| \longrightarrow 0 \quad \text { e } \quad \max _{x}\left|\lambda_{n C}(t)-\lambda_{0 C}(t)\right| \longrightarrow 0
$$

quando $m \longrightarrow \infty$ e $n \longrightarrow \infty$ mas $\frac{m}{n} \longrightarrow 0$.

Pelas suposições (A6-A9) e assumindo que $h_{1} \longrightarrow 0, h_{2} \longrightarrow 0$ e o número de intervalos seja $m=n^{v}$ com $0<v<1$ quando $n \longrightarrow \infty$, pode-se provar que:

(G1) quase certamente $\left\|\hat{\boldsymbol{\beta}}-\boldsymbol{\beta}_{0}\right\|_{2} \longrightarrow 0$ e $\left\|\hat{\boldsymbol{\phi}}-\boldsymbol{\phi}_{0}\right\|_{2} \longrightarrow 0$; e

(G2) quase certamente $\sup _{x \in\left[x_{(1)}, x_{(n)}\right]}\left|\hat{\lambda}_{n T}-\hat{\lambda}_{0 T}\right| \longrightarrow 0 \mathrm{e} \sup _{x \in\left[x_{(1)}, x(n)\right]}\left|\hat{\lambda}_{n C}-\hat{\lambda}_{0 C}\right| \longrightarrow 0$.

\subsection{Modelo Semiparamétrico II}

A presente seção foi escrita para apresentar o segundo modelo semiparamétrico que foi aplicado no exercício de simulação da seção 4.1. Xu et al. (2018) citaram o modelo de Huang e Zhang (2008) como exemplo para performance de ajuste a dados em que os tempos de falha e tempos de censura são gerados por intermédio de uma função de cópula.

Originalmente, a primeira diferença entre o modelo de Huang e Zhang (2008) e Xu et al. (2018) é que o primeiro possui maior liberdade quanto as funções de taxas de falha e de taxas de censura marginais

$$
\begin{aligned}
& \lambda_{T}\left(x_{i}\right)=\lambda_{0 T}\left(x_{i}\right) \exp \left\{\boldsymbol{Z}_{i}^{\top} \boldsymbol{\beta}\right\}, \\
& \lambda_{C}\left(x_{i}\right)=\lambda_{0 C}\left(x_{i}\right) \exp \left\{\boldsymbol{W}_{i}^{\top} \boldsymbol{\phi}\right\},
\end{aligned}
$$

no que diz respeito as covariáveis em que $Z=Z_{p \times 1}$ e $Z=W_{q \times 1}$ podem ser ou totalmente diferentes, ou parcialmente coincidentes ou idênticas — como no modelo de Xu et al. (2018). O texto aqui foi construído no caso particular ${ }^{1} W=Z_{p \times 1}$. A segunda diferença está nas funções basais $\lambda_{0 T}(x)$ e $\lambda_{0 C}(x)$. Enquanto em $X u$ et al. (2018) optaram por técnicas de aproximação linear, Huang e Zhang (2008) optaram por uma adaptação do estimador de Breslow para calcular as estimativas das funções basais acumuladas. Essa adaptação será explicada no decorrer do texto.

Com certeza a terceira e principal diferença entre os modelos desse capítulo está na metodologia em lidar com a presença de censura dependente no conjunto de dados efetivamente observável. Embora a premissa da distribuição conjunta de $T$ e $C$ também seja por uma família

\footnotetext{
${ }^{1}$ Embora também fosse possível generalizar o modelo de Xu et al. (2018) com matrizes de covariáveis distintas ou parcialmente coincidentes.
} 
membro da classe arquimediana, o cerne está na ideia de "redistribuição de massa" elaborada por Efron (1967).

Para um exercício de compreensão, primeiro considere que cada indivíduo em observação possui massa igual a 1 antes de falhar e 0 após o evento de interesse. No caso que a censura seja considerada independente, a massa de um indivíduo censurado é redistribuída uniformemente para todos os pontos do evento à direita. Suponha ainda uma amostra de tamanho $n$ de indivíduos que pertencem a um conjunto índice $\mathfrak{I}=\left\{i: x_{i}\right\}$ e que, para os tempos efetivamente observáveis $x_{i}$, não exista, convenientemente, empate tal que $x_{1}<x_{2}<\ldots<x_{n}$. Após o procedimento de redistribuição for feito para todos os indivíduos censurados de $x_{1}$ até $x_{n}$, a massa fica apenas sobre os tempos de falha. Então esse procedimento coincide com o estimador de Kaplan-Meier. Foi com essa aplicação de Efron que Zheng e Klein (1994) obtiveram estimadores autoconsistentes para as distribuições marginais a partir da distribuição conjunta de $T$ e $C$ assumindo uma cópula arquimediana. Então Huang e Zhang (2008) estenderam o resultado de Klein e Zheng para o modelo de taxas de falha. Aqui é discutido como Huang e Zhang construíram o modelo com base nessa aplicação de redistribuição de massa.

Suponha que o $i$-ésimo indivíduo seja censurado no tempo $x_{i}$, isto é, o indivíduo tem status $\delta_{C}\left(x_{i}\right)=1$. Então, questione-se: qual seria a probabilidade $P_{i}\left(x_{j}\right)$ do indivíduo $i$ falhar no tempo $x_{j}$, para cada $x_{j}>x_{i}$ ? A primeira observação é que existe uma dependência implícita entre a censura $C$ e o tempo de falha $T$ do $i$-ésimo indivíduo. Para o $i$-ésimo indivíduo, sejam $F_{T}$ a função de distribuição de $T$ e $F_{C}$ a função de distribuição de $C$. Com proeza matemática, Zheng e Klein (1994) — esse texto sugere ao leitor ler a demonstração — originalmente apresentaram o resultado

$$
\begin{aligned}
P_{i}\left(x_{j}\right) & =\operatorname{Pr}\left\{T \geq x_{j} \mid T \geq x_{i}, C=x_{i}\right\} \\
& =\frac{\operatorname{Pr}\left\{T \geq x_{j} \mid T \geq x_{i}, C=x_{i}\right\}}{\operatorname{Pr}\left\{T \geq x_{i} \mid C=x_{i}\right\}} \\
& =\frac{1-K_{2}\left(F_{T}\left(x_{j}\right), F_{C}\left(x_{i}\right)\right)}{1-K_{2}\left(F_{T}\left(x_{i}\right), F_{C}\left(x_{i}\right)\right)},
\end{aligned}
$$

em que $K_{2}(u, v)=\frac{\partial}{\partial v} K(u, v)$ e $K:[0,1]^{2} \longrightarrow[0,1]$ uma cópula arquimediana como discutido no Capítulo 2. Para obter o valor de (3.16), é necessário obter os estimadores das distribuições marginais $\hat{F}$ e $\hat{G}$. A primeira proposta para é um estimador autoconsistente. A segunda proposta é um estimador $A d H o c$ e, por último, Zheng (1992) apresentou um estimador arquimediano. Zheng e Klein (1994) construíram um estudo de simulação e destacaram que o terceiro estimador obteve o menor viés e erro quadrático médio relativo. Anteriormente, no mesmo ano de 1994 mas publicado no ano seguinte, Zheng e Klein (1995) mostram que uma cópula e um conjunto de dados efetivamente observáveis fornecem informação suficiente para identificar a distribuição marginal de $T$ e $C$. No caso que o $i$-ésimo indivíduo falha no instante 
$x_{i}$, analogamente, temos o estimador

$$
\begin{aligned}
Q_{i}\left(x_{j}\right) & =\operatorname{Pr}\left\{C \geq x_{j} \mid C \geq x_{i}, T=x_{i}\right\} \\
& =\frac{\operatorname{Pr}\left\{C \geq x_{j} \mid C \geq x_{i}, T=x_{i}\right\}}{\operatorname{Pr}\left\{C \geq x_{i} \mid T=x_{i}\right\}} \\
& =\frac{1-K_{1}\left(F_{T}\left(x_{j}\right), F_{C}\left(x_{i}\right)\right)}{1-K_{1}\left(F_{T}\left(x_{i}\right), F_{C}\left(x_{i}\right)\right)},
\end{aligned}
$$

sendo $K_{1}(u, v)=\frac{\partial}{\partial u} K(u, v)$, conforme também discutido no Capítulo 2. Entretanto, as quantidades descritas em 3.16 3.17) apresentam uma relação entre as probabilidades brutas $P_{i}\left(x_{j}\right)$ e $Q_{i}\left(x_{j}\right)$ com cópulas em função das distribuições marginais. Este trabalho, especificamente sobre cópula, foi desenvolvido para uma cópula em função de suas funções de sobrevivência marginais. Então levando em consideração (3.16 3.17), temos

$$
\left\{\begin{array}{l}
P_{i}\left(x_{j}\right)=\frac{K_{2}\left(S_{T}\left(x_{j}\right), S_{C}\left(x_{i}\right)\right)}{K_{2}\left(S_{T}\left(x_{i}\right), S_{C}\left(x_{i}\right)\right)} \\
Q_{i}\left(x_{j}\right)=\frac{K_{1}\left(S_{T}\left(x_{j}\right), S_{C}\left(x_{i}\right)\right)}{K_{1}\left(S_{T}\left(x_{i}\right), S_{C}\left(x_{i}\right)\right)}
\end{array} .\right.
$$

Originalmente, Huang e Zhang (2008) propuseram uma extensão ao modelo de taxas de falha proporcionais incluindo multiplicativamente aqueles estimadores $P_{i}\left(x_{j}\right)$ e $Q_{i}\left(x_{j}\right)$ para construir uma função de verossimilhança parcial estendida sob ideia sobre perda de massa de que o $i$-ésimo indivíduo perde no tempo $x_{j}$ novamente considerando que os tempos de falha (e censura) estão ordenados sem empates, Huang e Zhang (2008) argumentaram que a "perda de massa"da $i$-ésima observação no instante $x_{j}$ é

$$
D_{i}\left(x_{j}\right)=P_{i}\left(x_{j-1}\right)-P_{i}\left(x_{j}\right)
$$

e para o $i$-ésimo indivíduo que falha temos que $P_{i}\left(x_{j}\right)=1, \forall j \leq i$ e $P_{i}\left(x_{j}\right)=0$, caso contrário $(j>i)$. Pela perda de massa, temos que $D_{i}\left(x_{i}\right)=1$ e $D_{i}\left(x_{j}\right)=0, \forall j>i$. Portanto, quando um indivíduo que falha, ele contribui somente uma vez na verossimilhança estendida. De forma similar, para o $i$-ésimo indivíduo que é censurado, tem-se a perda de massa

$$
U_{i}\left(x_{j}\right)=Q_{i}\left(x_{j-1}\right)-Q_{i}\left(x_{j}\right)
$$

com $Q_{i}\left(x_{j}\right)=1, \forall j \leq i, U_{i}\left(x_{i}\right)=1$, e $Q_{i}\left(x_{j}\right)=0, U_{i}\left(x_{i}\right)=0, \forall j>i$. Para o $i$-ésimo indivíduo que é censurado sem dependência com o evento de interesse, temos que escrever $P_{i}\left(x_{j}\right)=Q_{i}\left(x_{j}\right)=$ $1, \forall j \leq i$ e $P_{i}\left(x_{j}\right)=Q_{i}\left(x_{j}\right)=1$, caso contrário, e $D_{i}\left(x_{j}\right)=U_{i}\left(x_{j}\right)=0$, para todo $j$. Huang e Zhang propuseram usar a função de verossimilhança parcial estendida dada por

$$
L(\boldsymbol{\beta}, \boldsymbol{\phi})=L_{T}(\boldsymbol{\beta}) L_{C}(\boldsymbol{\phi}),
$$


em que

$$
\left\{\begin{aligned}
L_{T}(\boldsymbol{\beta}) & =\prod_{j=1}^{n} \prod_{i=1}^{j}\left\{\frac{P_{i}\left(x_{j}\right) e^{\mathbf{Z}_{i}^{\top} \boldsymbol{\beta}}}{\sum_{k=1}^{n} P_{k}\left(x_{j}\right) \mathbf{Z}_{k}^{\top} \boldsymbol{\beta}}\right\}^{D_{i}\left(x_{j}\right)} \\
L_{C}(\boldsymbol{\phi}) & =\prod_{j=1}^{n} \prod_{i=1}^{j}\left\{\frac{Q_{i}\left(x_{j}\right) e^{\mathbf{Z}_{i}^{\top} \boldsymbol{\phi}}}{\sum_{k=1}^{n} Q_{k}\left(x_{j}\right) e^{\mathbf{Z}_{k}^{\top} \boldsymbol{\phi}}}\right\}^{U_{i}\left(x_{j}\right)}
\end{aligned}\right.
$$

Note que as funções são escritas de forma implícita $L_{T}(\boldsymbol{\beta})=L_{T}(\boldsymbol{\beta}, \boldsymbol{\phi})$ e $L_{C}(\boldsymbol{\phi})=L_{C}(\boldsymbol{\phi}, \boldsymbol{\beta})$ desde que fique claro que as quantidades $P_{i}\left(x_{j}\right)$ e $Q_{i}\left(x_{j}\right)$ dependem de ambos coeficientes de regressão $\beta$ e $\phi$. Prontamente, escreve-se a função log-verossimilhança

$$
l(\beta, \phi)=l_{T}(\beta)+l_{C}(\phi),
$$

com

$$
\begin{aligned}
& l_{T}(\boldsymbol{\beta})=\sum_{j=1}^{n} \sum_{i=1}^{j}\left\{D_{i}\left(x_{j}\right)\left[\log \left(P_{i}\left(x_{j}\right) e^{\mathbf{Z}_{i}^{\top} \boldsymbol{\beta}}\right)-S_{\text {pool }}(\boldsymbol{\beta})\right]\right\}, \\
& l_{C}(\boldsymbol{\phi})=\sum_{j=1}^{n} \sum_{i=1}^{j}\left\{U_{i}\left(x_{j}\right)\left[\log \left(Q_{i}\left(x_{j}\right) \mathrm{e}^{\mathbf{Z}_{i}^{\top} \boldsymbol{\phi}}\right)-S_{\text {pool }}(\boldsymbol{\phi})\right]\right\},
\end{aligned}
$$

e

$$
\begin{aligned}
& S_{\text {pool }}(\boldsymbol{\beta})=\log \left(\sum_{k=1}^{n} P_{k}\left(x_{j}\right) e^{\mathbf{Z}_{k}^{\top} \boldsymbol{\beta}}\right), \\
& S_{\text {pool }}(\boldsymbol{\phi})=\log \left(\sum_{k=1}^{n} Q_{k}\left(x_{j}\right) e^{\mathbf{Z}_{k}^{\top} \boldsymbol{\phi}}\right) .
\end{aligned}
$$

As funções escritas nas igualdades a cima também estão postas de forma implícita, pois ambas são uma soma de coeficientes que dependem dos estimadores $P_{i}\left(x_{j}\right)$ e $Q_{i}\left(x_{j}\right)$ e, por conseguinte, dependem dos ambos coeficientes de regressão $\beta$ e $\phi$. Huang e Zhang (2008) apresentaram um algoritmo para obter as estimativas dos parâmetros de regressão bem como a curva da função basal acumulada da função de log-verossimilhança resultante (3.19). Então é necessário resolver o seguinte problema de otimização

$$
\hat{\eta}=(\hat{\beta}, \hat{\phi})=\arg \max l(\beta, \phi)
$$

sendo

$$
\frac{\partial}{\partial \beta_{u}} l(\beta, \phi)=0 \quad \text { e } \quad \frac{\partial}{\partial \phi_{u}} l(\beta, \phi)=0 .
$$

Devido as igualdades a cima, é necessário um algoritmo iterativo para obter $\hat{\eta}$. Também outro motivo é pela presença de quantidades não conhecidas como $P_{i}\left(x_{j}\right)$ e $Q_{i}\left(x_{j}\right)$. O algoritmo proposto está disponível no Quadro 2 e os detalhes de cada passo são descritos a seguir. 


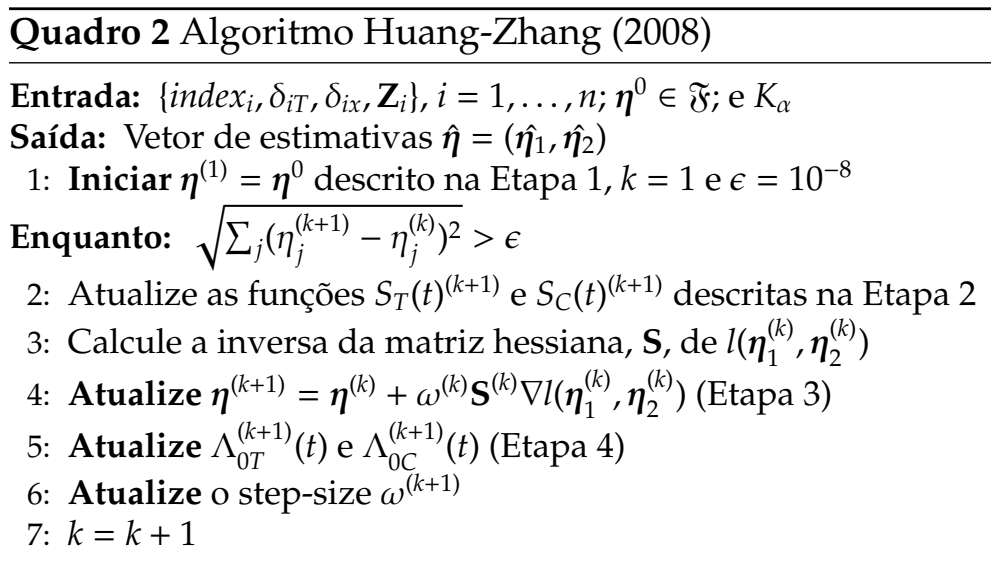

Fim Enquanto

Etapa 1. Assumindo o mecanismo de censura independente, para o primeiro passo, utilize o ajuste por modelo de Cox convencional para obter $\hat{\boldsymbol{\beta}}^{(0)}$ e $\hat{\boldsymbol{\phi}}^{(0)}$ como as estimativas iniciais de $\boldsymbol{\beta}$ e $\phi$. Então aplica-se o método de Breslow para obter $\hat{\Lambda}_{0 T}\left(x_{j}\right)$ e $\hat{\Lambda}_{0 C}\left(x_{j}\right)$ como as estimativas de $\Lambda_{0 T}\left(x_{j}\right)$ e $\Lambda_{0 C}\left(x_{j}\right)$;

Etapa 2. Para $i=1,2, \ldots, n$, calcule

$$
\begin{aligned}
S_{T}^{(k)}(t) & =\exp \left\{-\hat{\Lambda}_{0 T}^{(k)}(t) \exp \left\{\mathbf{Z}_{i}^{\top} \hat{\boldsymbol{\beta}}^{(k)}\right\}\right\}, \\
S_{C}^{(k)}(t) & =\exp \left\{-\hat{\Lambda}_{0 C}^{(k)}(t) \exp \left\{\mathbf{Z}_{i}^{\top} \hat{\boldsymbol{\phi}}^{(k)}\right\}\right\}
\end{aligned}
$$

Então para cada $j$ tal que $x_{j}>x_{i}$, atualize

$$
\begin{aligned}
P_{i}^{(k)}\left(x_{j}\right) & =\frac{K_{2}\left(S_{T}^{(k)}\left(x_{j}\right), S_{C}^{(k)}\left(x_{i}\right)\right)}{K_{2}\left(S_{T}^{(k)}\left(x_{i}\right), S_{C}^{(k)}\left(x_{i}\right)\right)}, \\
Q_{i}^{(k)}\left(x_{j}\right) & =\frac{K_{1}\left(S_{T}^{(k)}\left(x_{j}\right), S_{C}^{(k)}\left(x_{i}\right)\right)}{K_{1}\left(S_{T}^{(k)}\left(x_{i}\right), S_{C}^{(k)}\left(x_{i}\right)\right)} .
\end{aligned}
$$

Etapa 3. Atualize todas as quantidades estimadas a cima na função de log-verossimilhança e depois calcule os zeros das suas derivadas parciais de primeira ordem com respeito a $\hat{\beta}_{u}^{(k+1)}$ e $\hat{\phi}_{u}^{(k+1)}$, respectivamente, via algum método iterativo;

Etapa 4. Utilizado $\hat{\beta}_{u}^{(k+1)}, \hat{\phi}_{u}^{(k+1)}$ e as outras quantidades estimadas anteriores, calcule os estimadores de Breslow

$$
\begin{aligned}
& \hat{\Lambda}_{0 T}^{(k+1)}\left(x_{i}\right)=\sum_{j: x_{j} \leq x_{i}} \frac{\sum_{i: x_{i} \leq x_{j}} D_{i}^{(k)}\left(x_{j}\right)}{\sum_{k=1}^{n} P_{i}^{(k)}\left(x_{j}\right) \exp \left\{\mathbf{Z}_{i}^{\top} \hat{\beta}_{u}^{(k+1)}\right\}}, \\
& \hat{\Lambda}_{0 \mathrm{C}}^{(k+1)}\left(x_{i}\right)=\sum_{j: x_{j} \leq x_{i}} \frac{\sum_{i: x_{i} \leq x_{j}} U_{i}^{(k)}\left(x_{j}\right)}{\sum_{k=1}^{n} Q_{i}^{(k)}\left(x_{j}\right) \exp \left\{\mathbf{Z}_{i}^{\top} \hat{\phi}_{u}^{(k+1)}\right\}},
\end{aligned}
$$


Etapa 5. Atualize para o próximo passo e volte para a Etapa 2 até atingir-se algum critério de convergência do método iterativo.

Tanto o gradiente e matriz hessiana do modelo resultante (3.19) ficaram disponíveis apenas no Apêndice B (incluindo demonstrações) pois suas expressões são demasiadamente extensas. Em Huang e Zhang (2008) foi apresentado um estudo de simulação e aqui nesse texto também será apresentado no próximo capítulo um estudo semelhante. Por fim, ressaltam-se dois pontos importantes:

(3.5.1) Quando se utiliza o modelo com uma cópula assumindo independência entre $T$ e $C$, a função de log-verossimilhança parcial não se reduz a log-verossimilhança parcial de Cox; e

(3.5.2) esse modelo possui uma função de log-verossimilhança que tem um método de estimação computacionalmente custoso. No próximo capítulo será melhor explicado essa observação.

Huang e Zhang (2008) afirmam que os estimadores dos coeficientes de regressão, $\hat{\beta}$ e $\hat{\phi}$, e das funções basais acumuladas, $\widehat{\Lambda_{0 T}}$ e $\widehat{\Lambda_{0 C}}$, são autoconsistentes pela definição de Efron (1967). Os autores ainda defenderam evidências em estudos prévios sobre propriedades assintóticas em um contexto sem regressão e foram enfáticos no seu argumento que seu estudo de simulação apresentou resultado satisfatório de convergência. Entretanto, admitiram que era necessário uma investigação teórica mais profunda.

Por ora, pelo fato em (3.5.2), significa em (3.5.1) que esse modelo não é competitivo quanto a performance contra a função de log-verossimilhança seja a parcial de Cox (independente) ou a função em Xu et al. (2018). No próximo capítulo essa afirmação terá melhor sustentação pelo estudo de simulação. 
$\prod_{\text {Capítulo }}$

\section{Simulações}

Nesse capítulo são apresentados os resultados de um estudo de simulação. Foram feitos dois estudos de simulação com os seguintes objetivos: o primeiro estudo foi realizado para avaliar a performance dos procedimentos de estimação em Xu et al. (2018) e em Huang e Zhang (2008) quanto aos métodos de estimação propostos em específicos cenários. Para todos cenários, os dados são gerados de acordo com o modelo de Cox sob uma dependência funcional entre o tempo de falha e tempo de censura. Como discutido em capítulos anteriores, essa dependência é produto de uma predeterminada cópula na função de sobrevivência bivariada. O segundo estudo tem o compromisso de avaliar uma aplicabilidade às abordagens discutidas no Capítulo 3 para o ajuste do Tempo de Sobrevivência Ajustado pela Qualidade de Vida (TSAQV), conforme a motivação deste trabalho.

\subsection{Tempos de Sobrevivência: uma Análise de Sensibilidade}

Conforme já mencionado, esse primeiro estudo de simulação tem como objetivo principal avaliar as metodologias apresentadas, bem como compará-las. Para isso, foram gerados dados em que o tempo de falha e o tempo de censura são dependentes, variando-se tamanho amostral, proporção de censura e o valor do coeficiente de dependência.

A primeira questão que surge é com relação a geração de tempos de falha e censura que sejam dependentes. É, para tanto, necessário introduzir no par aleatório $\left(T_{i}, C_{i}\right)$ uma dependência entre seus componentes tempo de falha $T_{i}$ e tempo de censura $C_{i}$ pertencentes ao $i$-ésimo indivíduo. Uma ferramenta aqui também utilizada para embutir essa dependência no par é aplicada pela relação entre uma predeterminada cópula com a função de sobrevivência do par $\left(T_{i}, C_{i}\right)$.

Portanto, cópula previamente definida, é orientado o uso de uma família membro arquimediana com o parâmetro $\alpha$ fixado e conhecido. É preciso lembrar que essa específica formulação neste texto foi desenvolvida apenas para três famílias membros da classe arquimediana. Sobre as funções basais do tempo de falha $T_{i}$ e tempo de censura $C_{i}$, serão usadas as variações descritas na Tabela 4.1 . 
Tabela 4.1: Distribuições das basais do tempo de falha e censura

\begin{tabular}{c|cc}
\hline \hline Funções basais & I & II \\
\hline Falha & $\lambda_{0 T}(x)=\frac{x}{2}$ & $\lambda_{0 T}(x)=\frac{8 x}{25}$ \\
Censura & $\lambda_{0 C}(x)=\frac{1}{5}$ & $\lambda_{0 C}(x)=\frac{1}{3}$ \\
\hline \hline
\end{tabular}

No decorrer do texto, as distribuições basais I e II na Tabela 4.1 podem ser nomeadas por Esquema I e Esquema II, respectivamente. A princípio, foi repetido e estudado os cenários isolados por Xu et al. (2018) tal que o Esquema I descrito acima é um daqueles cenários. De todo modo, não foi seguido o mesmo estudo de simulação e, no decorrer do capítulo, vai ser explicado passo a passo os motivos para a escolha dos esquemas I e II. Antes de prosseguir com os detalhes dos esquemas, é importante destacar como é utilizada uma cópula para gerar um par aleatório $(t, c)$ com um nível de dependência $\tau$ fixado. O procedimento adotado foi o seguinte:

(E1) gere um número $v_{2}$ de $V_{2} \sim U(0,1)$;

(E2) dado $\left.\frac{\partial}{\partial x} K_{\alpha}\left(v_{1}, x\right)\right|_{x=v_{2}}$, gere um número $v_{1}$ de $V_{1} \mid V_{2}=v_{2}$;

(E3) dado um par gerado $\left(v_{1}, v_{2}\right)$, obtenha a inversa de $t=S_{T}^{-1}\left(1-v_{1}\right)$ e $c=S_{C}^{-1}\left(1-v_{2}\right)$.

A técnica de simulação é baseada intrinsecamente no método de simulação da inversa para um par aleatório (veja Robert et al., 2010) com adendo da cópula no passo (2) que requer a aplicação do resultado (2.8), inserindo a dependência entre as variáveis $T_{i}$ e $C_{i}$. Sem o passo (E2) para gerar $v_{1}$, seria necessário escrever a função de distribuição de $V_{1} \mid V_{2}=v_{2}$ podendo ser inconveniente por talvez não ter uma expressão analítica ou que não permita administrar o nível de dependência. Contudo, devido a outra ótima propriedade para uma cópula membro da classe arquimediana, a função de distribuição de $V_{1} \mid V_{2}=v_{2}$ é igual a derivada de primeira ordem da cópula $K_{\alpha}\left(v_{1}, x\right)$ com relação a $x$ no ponto $x=v_{2}$, veja os resultados (2.8) e (2.9). O último passo é apenas uma simples aplicação da inversa das funções de sobrevivência dos tempo de falha e tempo de censura de acordo com as basais da Tabela 4.1.

Então um par gerado $(t, c)$ acima é considerado um par gerado de $(T, C)$. Como o interesse é gerar um par partindo de um modelo de taxas de falha proporcionais, então as funções de sobrevivência do tempo de falha e tempo de censura são descritas com

$$
S_{T}\left(t_{i}\right)=\exp \left\{-\Lambda_{0 T}\left(t_{i}\right) e^{Z_{i}^{\top} \beta}\right\}
$$

$\mathrm{e}$

$$
S_{C}\left(c_{i}\right)=\exp \left\{-\Lambda_{0 C}\left(c_{i}\right) e^{Z_{i}^{\top} \phi}\right\} .
$$

É necessário gerar as covariáveis como vetores não nulos escolhidas como $Z_{i 1} \sim \operatorname{Ber}(0.5)$ e $Z_{i 2} \sim U(-2,2)$. Na segunda parte do capítulo, será usada outra distribuição. Por último, para todos os cenários de simulação, foram usados os seguintes coeficientes de regressão $\beta=(-0,5 ; 0,1)^{\top}$ e $\phi=(0,3 ; 0,2)^{\top}$. 
Vale lembrar que o par gerado $\left(t_{i}, c_{i}\right)$ não é um par observável, pois não se pode observar o tempo de censura verdadeiro quando um indivíduo em observação sofreu falha e viceversa. Então o tempo efetivamente observável daquele indivíduo é definido por $x_{i}=\min \left(t_{i}, c_{i}\right)$ formando o par observável $\left(x_{i}, \delta_{T}\left(x_{i}\right)\right)$ com a função indicadora $\delta_{T}\left(x_{i}\right)=I\left(t_{i}<c_{i}\right)$. Entretanto, um indivíduo em observação também pode ser passivo de censura por um evento que não esteja relacionado com o evento de interesse por uma estrutura de dependência. Então a presença do fenômeno de censura independente também é razoável mesmo para com aquele estudo que considere a premissa de que sua censura seja dependente. Nessa simulação, acrescentou-se também uma porção de censura independente.

Adiante, será apresentado uma análise exploratória por gráficos para explicar o fenômeno das proporções e seu porquê, por exemplo, em um estudo se pode obter que $7 \%$ dos pacientes estão sob censura independente dado certas variáveis independentes mas, por outro lado, poderia ser obtido $14 \%$ para outra variáveis. Então a tripla não observável $\left(t_{i}, c_{i}, a_{i}\right)$ é gerada pela tripla aleatória $\left(T_{i}, C_{i}, A_{i}\right)$, com $A_{i}$ sendo o tempo de censura independente formando, assim, a tripla observável $\left(x_{i}, \delta_{T}\left(x_{i}\right), \delta_{C}\left(x_{i}\right)\right)$ sendo $x_{i}$ o tempo e os indicadores $\delta_{T}\left(x_{i}\right)=I\left(t_{i}<c_{i}\right)$ e $\delta_{C}\left(x_{i}\right)=I\left(t_{i}>c_{i}\right)$.

Xu et al. (2018) produziram somente 3 cenários modificando um único parâmetro na basal do tempo de falha por duas constantes, o nível de dependência $\tau$ também por duas constantes e variando apenas o tamanho amostral $n=200$ e $n=1000$. Quanto aos níveis de dependência, eles escolheram cenários com falha e censura fortemente dependentes $\tau=0,80$ e fracamente $\operatorname{com} \tau=0,30$. Levando em consideração só as proporções de falha e censura, eles usaram cenários bastante balanceados entre $\pi_{F}$ e $\pi_{C}$. Porém, levando em consideração a proporção de censurados independentemente, todos os cenários ficaram pendendo maior proporção para censura geral. Assim, ficou decidido para esse texto usar o estudo de simulação em Xu et al. (2018) como alicerce para verificar o comportamento das estimativas sob um novo fenômeno com cenários desbalanceados favoráveis a falhas e também a censuras. Por outro ponto de vista, tem-se o interesse de estudar a performance com cenários sob proporção moderada ( funções basais I) e fortemente censurada (funções basais II). Por exemplo, olhando a Figura 4.1, tem-se 4 cenários modificando somente o tamanho amostral. Sem precisão, uma das possíveis conclusões que o leitor pode pensar é que, aparentemente, o desbalanceamento é favorável ao evento de interesse sendo que a proporção de censura total fica algo em torno de $35 \%$.

A seguir, são apresentados cinco exemplos em figuras contendo cada uma quatro amostras de tamanhos $n$ com $T$ e $C$ fracamente dependentes $(\tau=0,20)$. O conjunto das triplas $\left\{\left(t_{i}, c_{i}, a_{i}\right): i=1,2, \ldots, n\right\}$ são gerados de forma independente e são ordenados crescente para o conjunto $\left\{x_{i}=\min \left(t_{i}, c_{i}, a_{i}\right): x_{(1)}<x_{(2)}<\ldots<x_{(n)}\right\}$. A Figura 4.2 apresenta 4 gráficos para aquelas réplicas com suas proporções de falha e censura dependente. Nota-se que as proporções são razoavelmente semelhantes mesmo com o aumento significativo do tamanho de cada réplica. Por outro lado, é interessante verificar como se comportar a simulação quando é aumentado a dependência entre $T$ e $C$. A Figura 4.3 apresenta 4 réplicas sob o mesmo esquema anterior excetuando que o coeficiente de Kendall agora é quatro vezes mais forte $(\tau=0,80)$. A nítida mudança é na diminuição na proporção de dados efetivamente observáveis que so- 

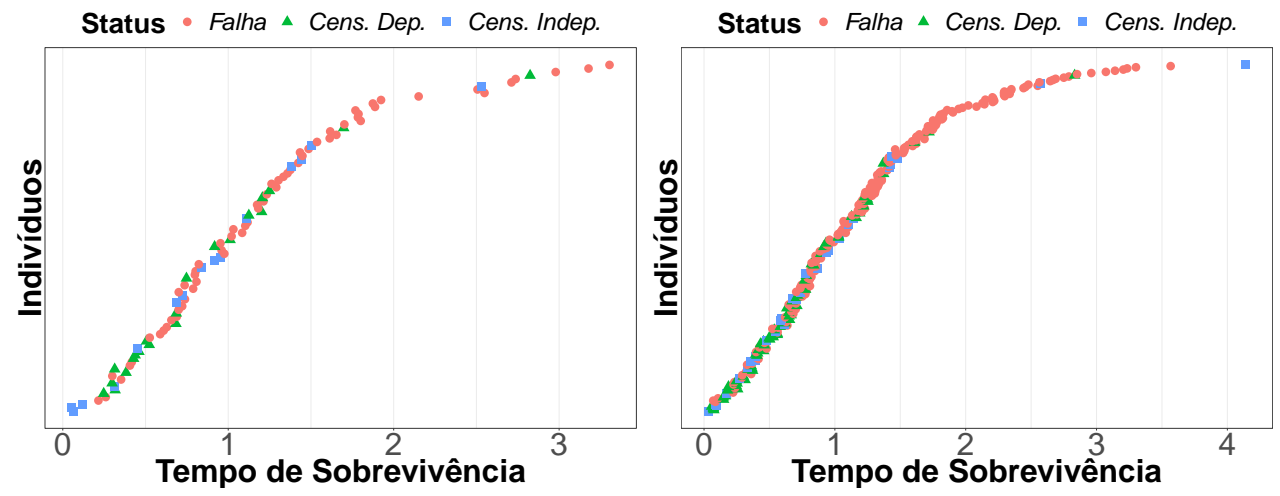

(a) $n=100, \pi_{F}=64 \%$ e $\pi_{C}=21 \%$

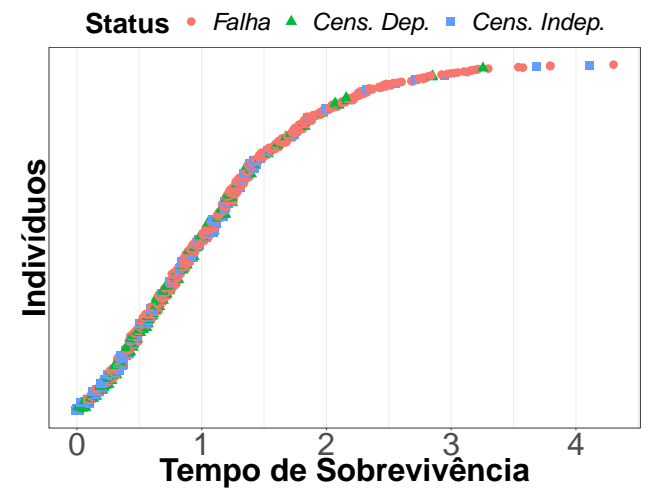

(b) $n=300, \pi_{F}=67 \%$ e $\pi_{C}=22.67 \%$

Status - Falha $\triangle$ Cens. Dep. - Cens. Ind.

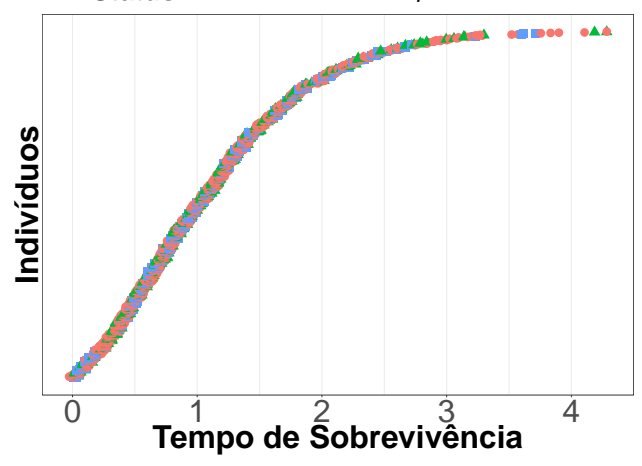

(c) $n=700, \pi_{F}=62.71 \%$ e $\pi_{C}=23.23 \%$

(d) $n=1300, \pi_{F}=63.92 \%$ e $\pi_{C}=23.23 \%$

Figura 4.1: Réplicas de tamanho $n$ para $T$ e $C$ fracamente dependentes

freram censura dependente. Novamente houve uma razoável estabilidade às proporções de falha e censura a respeito do aumento do tamanho $n$ da amostra. Outro aspecto é sobre as funções basais. Funções basais semelhantes geralmente propiciam um melhor balanceamento das proporções. As réplicas no cenário da Figura 4.2 apresentam um moderado desbalanceamento amostral das proporções, já as réplicas no cenário da Figura 4.3 apresentam um severo desbalanceamento amostral das proporções. Huang e Zhang (2008) comentam que esses tipos de cenários contribuem para uma maior dificuldade na obtenção das estimativas dos coeficientes de regressão do evento de interesse $T$ já que eles estarão funcionalmente dependentes (nos modelos adotados) dos coeficientes de regressão da censura $C$. Menos informação de uma proporção para com outra aumenta o viés. A situação se agrava a medida que, em uma análise de dados reais, uma amostra pode não ser grande o suficiente. Como exemplo, os gráficos em $4.1 \mathrm{a}$ e $4.2 \mathrm{a}$ são das réplicas de tamanho $n=100$ com proporções de censura iguais a $22 \%$ e $11 \%$, respectivamente.

A Figura 4.4 apresenta 4 novas réplicas agora aplicando uma censura à direita do tipo I. É visível que a aplicação da censura do tipo I provocou um aumento na proporção de falha resultando em um maior desbalanceamento nas proporções. Outro ponto importante é o cuidado com o tamanho amostral. No gráfico 4.4a da Figura 4.4, tem-se uma réplica de tamanho $n=100$ em que nenhum indivíduo sofreu a censura do tipo I. Quando se aumenta o tamanho amostral, os gráficos seguintes mostram que alguns indivíduos começam a ficarem 

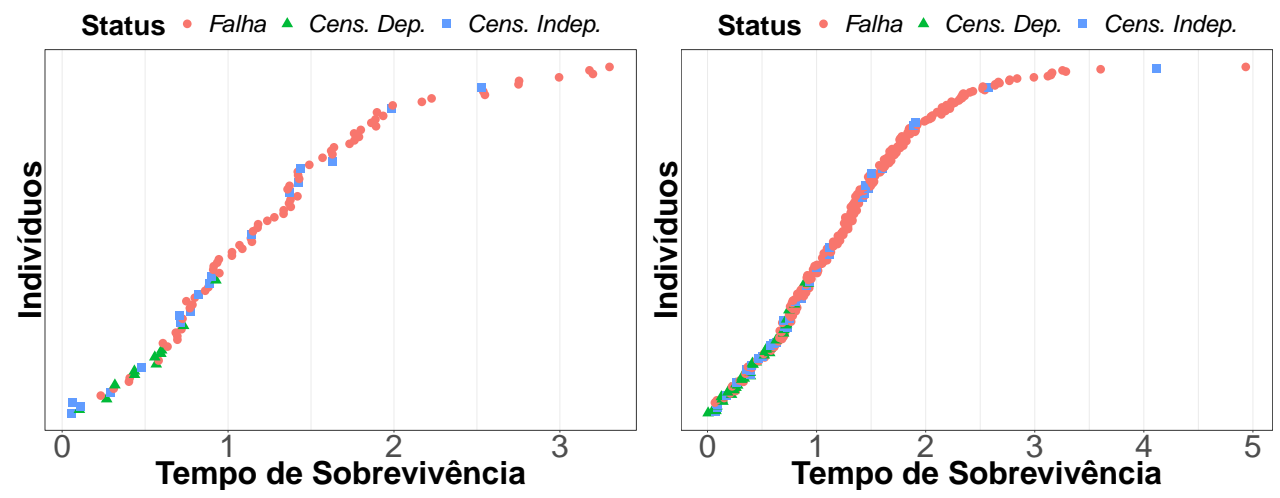

(a) $n=100, \pi_{F}=71 \%$ e $\pi_{C}=11 \%$

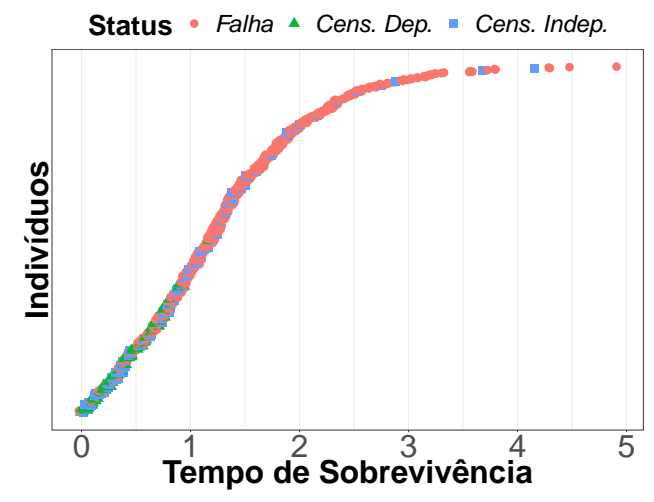

(b) $n=300, \pi_{F}=75 \%$ e $\pi_{C}=13 \%$

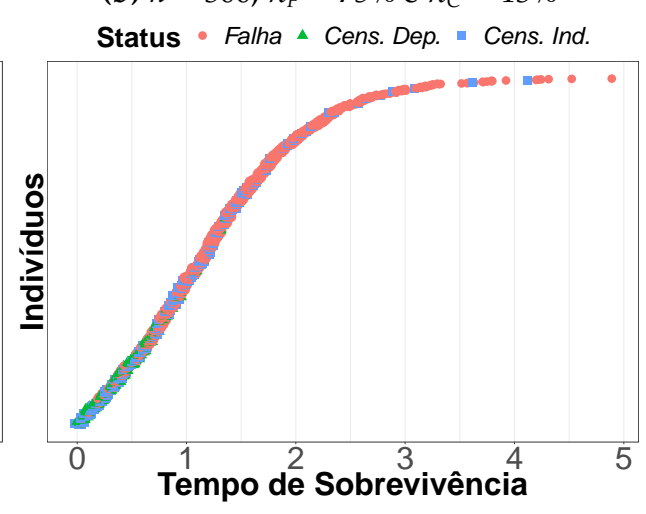

(c) $n=700, \pi_{F}=71,29 \%$ e $\pi_{C}=13,86 \%$

(d) $n=1300, \pi_{F}=71,62 \%$ e $\pi_{C}=14,31 \%$

Figura 4.2: Réplicas de tamanho $n$ para $T$ e $C$ fortemente dependentes

passivos à censura do tipo $\mathrm{I}$.

A Figura 4.5 apresenta as 4 e últimas réplicas para simulações em que $T$ e $C$ são fortemente dependentes $(\tau=0.80)$, mas com nova função basal para $C$ dada por $\lambda_{0 C}(x)=\frac{2}{5}$. Esse alteração provoca diretamente o novo balanceamento amostral entre as proporções de falha e censura. Entretanto, essa modificação ainda não é suficiente para resolver o problema concentração de dados censurados mais a esquerda. Na tripla artificial $\left(x, \delta_{T}(x), \delta_{C}(c)\right)$, a ocorrência de falha é predominante e dispersa à linha do tempo mas a ocorrência de censura é aglutinadora para valores à esquerda da média do tempo de sobrevivência.

Toda essa análise descritiva acerca do balanceamento amostral das proporções de falha e censura bem como a preocupação sobre dispersão do subconjunto de indivíduos sob um mesmo status, foi necessária para explicar o motivo desse texto ter escolhido a administração do mecanismo de censura do tipo I como também para já explicitar o que pode se esperar dos resultados das simulações. Visando esses resultados, uma alternativa é corrigir aquela possibilidade de desbalanceamento e falta de dispersão utilizando o resultado apresentado na subseção 3.4.1 - por uma abordagem via piecewise e também $M$-splines - em que é adotado uma construção de duas partições distintas usando os percentis dos indivíduos com status de falha e censura como base para criar intervalos com de forma balanceada às proporções. Essa construção de intervalos visa equilibrar a proporção de indivíduos com mesmo status para cada intervalos. Em um exercício para a réplica $n=100$ e $m=3$, tem-se a seguinte 

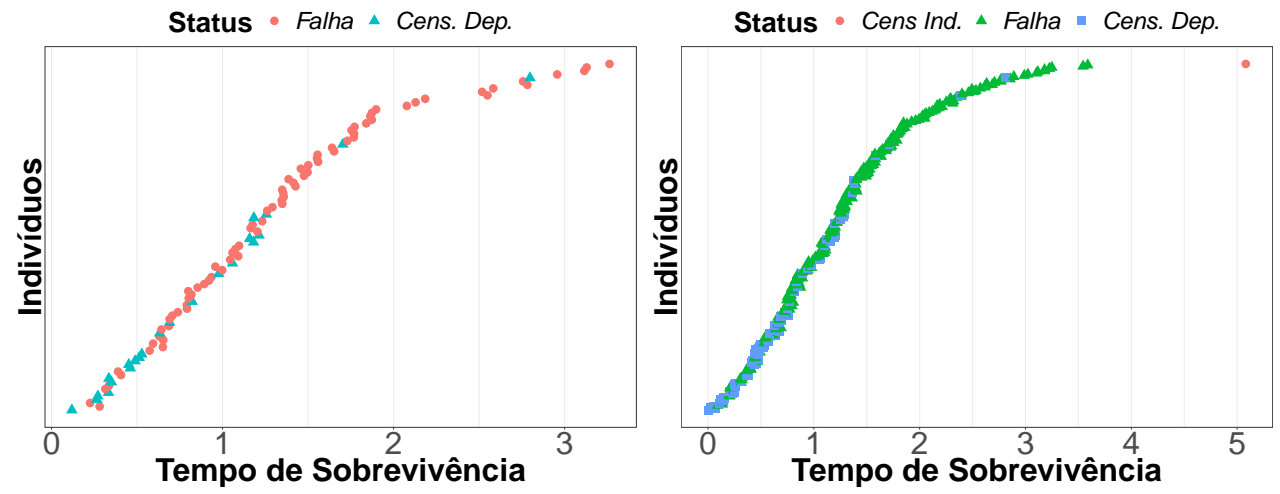

(a) $n=100, \pi_{F}=77 \%$ e $\pi_{C}=23 \%$

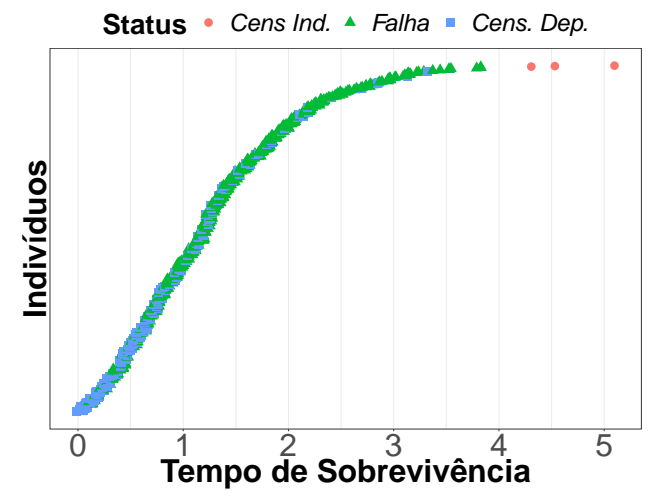

(b) $n=300, \pi_{F}=74.67 \%$ e $\pi_{C}=25 \%$

Status - Cens Ind. $\triangle$ Falha $\|$ Cens. Dep.

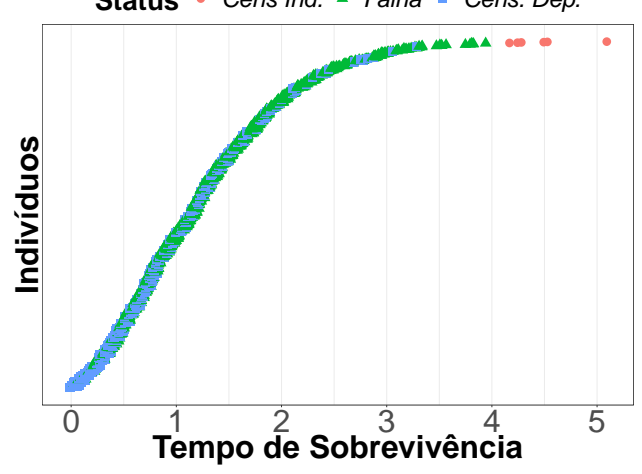

(c) $n=700, \pi_{F}=72.71 \%$ e $\pi_{C}=26.86 \%$

(d) $n=1300, \pi_{F}=73.46 \%$ e $\pi_{C}=26.08 \%$

Figura 4.3: Novas Réplicas para $T$ e $C$ Fracamente Dependentes sob Censura do Tipo I

divisão: a primeira intervalos recebe 12 indivíduos censurados, a segunda recebe 14 indivíduos censurados e a terceira intervalos recebe 12 indivíduos censurados. O que resulta em uma proporção bastante razoável diferentemente do primeiro exemplo. Agora, um problema quanto a esse procedimento é a possibilidade das intervalos nos extremos serem preenchidas por muitos indivíduos de outros status. Nesse último exemplo, na partição da censura, as intervalos terão os seguintes números totais de indivíduos são 14, 21 e 65. Isto é, a primeira intervalos é ocupada por 12 indivíduos censurados mais 2 indivíduos que falharam e a última intervalos tem 53 indivíduos de toda sorte para além dos censurados. Esse fato pode também provocar um aumento no viés.

Xu et al. (2018) decidiram usar uma partição para o conjunto de tempos de sobrevivência sem discriminar seus status (falha e censura dependente ou independente), priorizando um balanceamento estável para o número de indivíduos pertencentes aos sucessivos e disjuntos intervalos. Sem levar em consideração o status, não é raro a partição apresentar alguns subconjuntos sem um único indivíduo de status de falha ou mesmo de censura dependente. Com esse fenômeno, em $m$ intervalos, algumas estimativas $\hat{\theta}_{m} \mathrm{e} \hat{\gamma}_{m}$ são iguais a zero podendo gerar uma singularidade na matriz de informação. Esse fato foi discutido e apresentado uma solução por eles. Entretanto, nesse texto a partição foi tomada diferente. Foi priorizado o balanceamento estável de indivíduos por status nos sucessivos intervalos. No Capítulo 3, foi apresentado a construção de duas distintas partições: uma para falha e a outra para censura. 


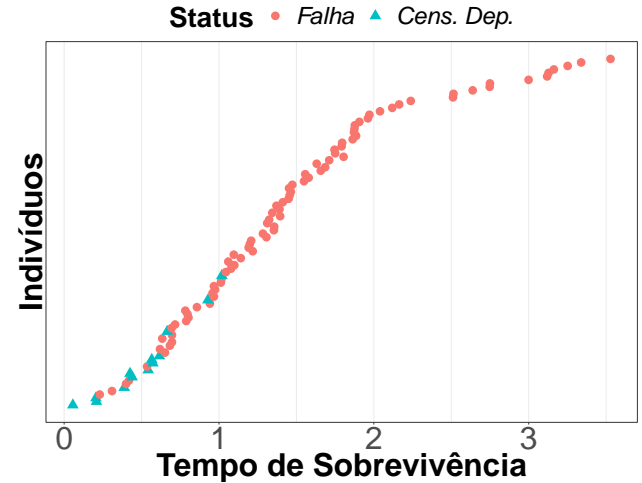

(a) $n=100, \pi_{F}=87 \%$ e $\pi_{C}=13 \%$

Status - Cens Ind. A Falha = Cens. Dep.

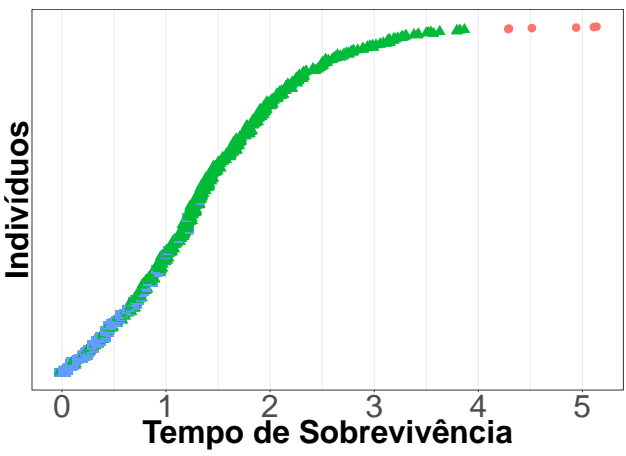

(c) $n=700, \pi_{F}=84.14 \%$ e $\pi_{C}=15 \%$

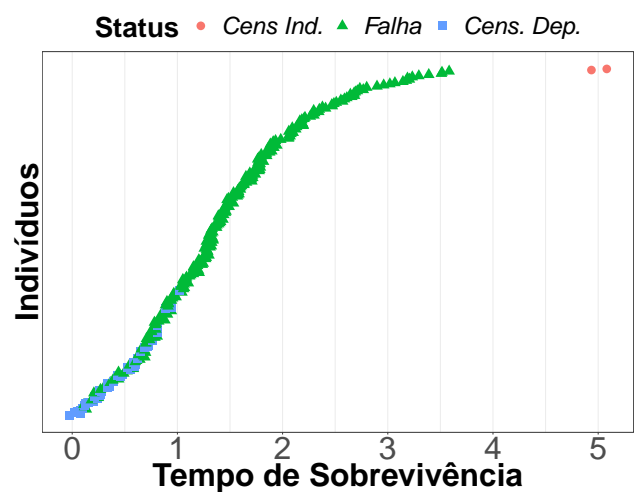

(b) $n=300, \pi_{F}=85.67 \%$ e $\pi_{C}=13.67 \%$

Status - Cens Ind. $\triangle$ Falha $=$ Cens. Dep.

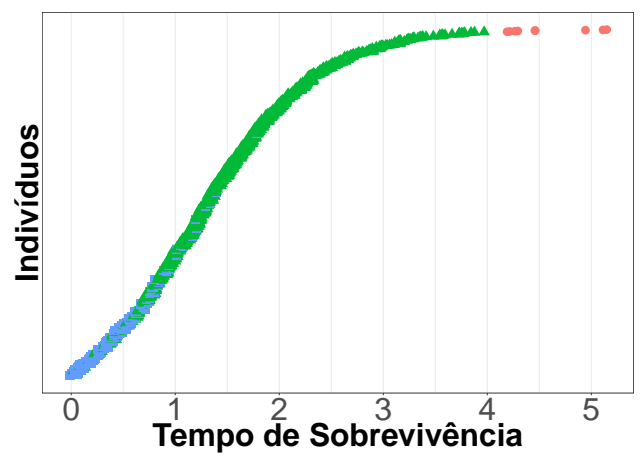

(d) $n=1300, \pi_{F}=84.15 \%$ e $\pi_{C}=15.15 \%$

Figura 4.4: Novas Réplicas para $T$ e $C$ Fortemente Dependentes sob Censura do Tipo I

Para finalizar essa seção prosseguindo com os resultados das simulações, faz-se necessário explicar o quadro de esquematização escolhido nesse trabalho. O quadro, um tanto extenso, apresenta uma razoável análise para entender as nuances entre no nível de dependência e níveis de proporção de censura dependente.

A Figura 4.6 apresenta uma esquematização genérica para representar os Esquemas I e II. Ao longo desse texto foi enfaticamente alertado que só foram utilizados apenas três famíliamembros da classe arquimediana. Entretanto, para as simulações, só duas foram utilizadas: Clayton e Frank. Prosseguindo, para cada família-membro, foram desenvolvidos esquemas com o mesmo quadro. Então, por exemplo, a cópula Clayton seguiu Esquema I e Esquema II, tamanho amostral $n=300$ e $n=1000$, níveis de dependência $\tau(0 ; 0,50 ; 0,80)$.

Foram 24 cenários gerados variando família membro, tamanho amostral, esquema e nível de dependência. Para cada cenário foi estimado o vetor $\eta$ com a verossimilhança de Xu et al. (2018) com três valores fixos para $\tau$, totalizando 72 processos de simulações. Levando em consideração que foram usadas três abordagens para aproximação das funções basais (paramétrico, piecewise e M-splines), então o total parcial de processos de estimação vai para 216. Por último, considerando que para todos os cenários foram realizados $N=1300$ réplicas, o número total de processos de estimação vai para 280800. Também foi utilizado a verossimilhança de Huang e Zhang (2008) mas com apenas seis cenários variando somente o tamanho amostral e nível de dependência. Isso foi feito porque Huang e Zhang relataram que seu processo de limitação é 

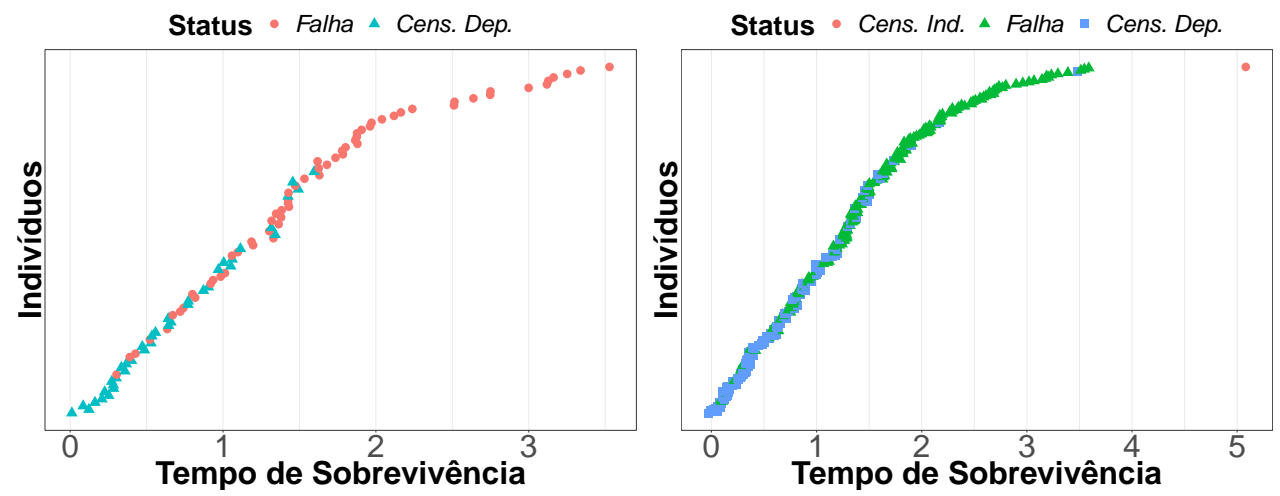

(a) $n=100, \pi_{F}=62 \%$ e $\pi_{C}=38 \%$

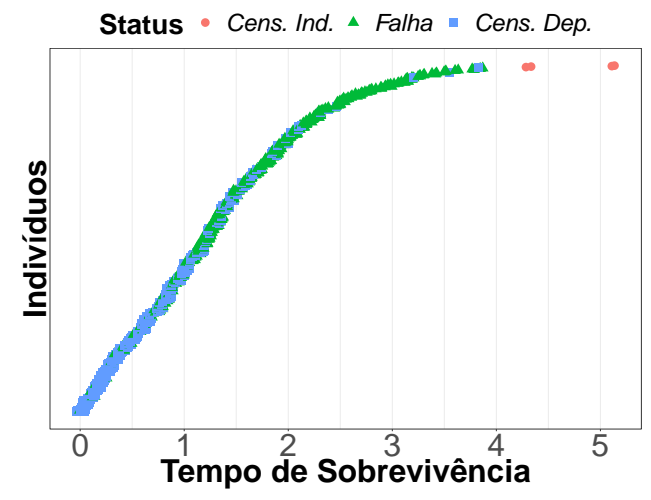

(b) $n=300, \pi_{F}=60 \%$ e $\pi_{C}=39,67 \%$

Status - Cens. Ind. $\triangle$ Falha $=$ Cens. Dep.

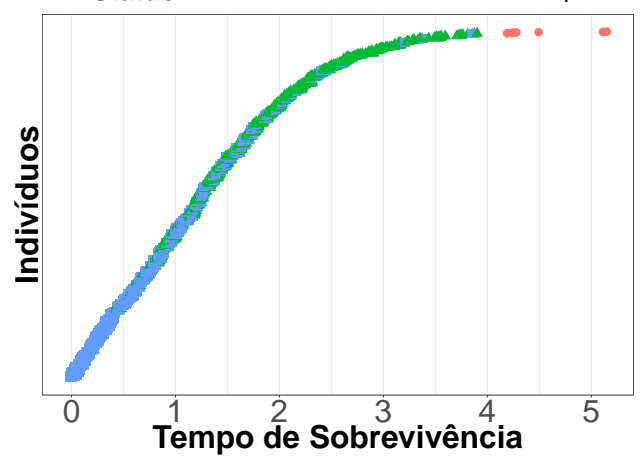

(c) $n=700, \pi_{F}=57 \%$ e $\pi_{C}=42,43 \%$

(d) $n=1300, \pi_{F}=55,54 \%$ e $\pi_{C}=43,92 \%$

Figura 4.5: Novas Réplicas para $T$ e $C$ com Proporções Balanceadas

computacionalmente lento, tornando proibitivo um estudo de simulação mais extenso. Ainda sobre as simulações utilizando as funções log-verossimilhança (3.13) e (3.19), elas foram analisadas com as mesmas medidas que Xu et al. (2018) utilizaram: Viés $B\left(\hat{\beta}_{j}\right)$, Erro Padrão via Monte Carlo $\hat{\theta}_{M C}\left(\hat{\beta}_{j}\right)$, Erro Padrão Assintótico $\hat{\theta}_{A S}\left(\hat{\beta}_{j}\right)$ e uma Probabilidade de Cobertura (CP) a um nível de confiança de $96 \%$. Todos resultados estão disponíveis no Apêndice $\mathrm{C}$ em formato de tabelas.

\subsubsection{Resultados}

A presente subseção apresenta os principais resultados das estimativas para cada cenários com destaque para abordagem de aproximação linear às funções basais dos tempos de falha e censura bem como tamanho amostral. Como dito, as estimativas foram analisadas sob a performance obtida pelas medidas: Viés $B\left(\eta_{j}\right)$, Erro Padrão via Monte Carlo $\overline{\hat{\theta}}_{M C}\left(\hat{\eta}_{j}\right)$, Erro Padrão Assintótico $\bar{\theta}_{A S}\left(\hat{\eta}_{j}\right)$, o Erro Quadrático Médio (EQM) e uma Probabilidade de Cobertura (CP) com nível de $96 \%$. Com o número consideravelmente elevado de cenários, não é viável apresentar todos os resultados de uma vez só em formato de tabelas. Também foram calculados as estimativas com base na premissa de censura independente. Foram obtidos erros quadráticos médios $\operatorname{EQMVP}\left(\eta_{j}\right)$ e o valores absolutos dos vieses $\operatorname{VAVVP}\left(\eta_{j}\right)$. Os principais resultados serão apresentados em gráficos. De todo modo, todos os cenários são também descritos em formato 


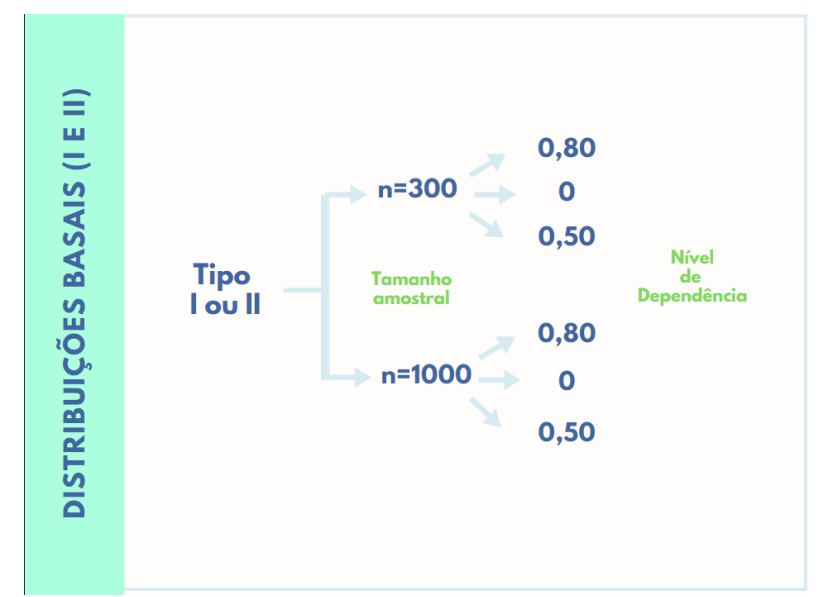

Figura 4.6: Esquematização das distribuições basais para simulação

de tabela e estão disponíveis no Apêndice C.

Para as abordagens de aproximação linear às funções basais, é preciso destacar a abordagem por piecewise que é um método de aproximação por $m$ constantes ao longo do tempo referidos para cada intervalo das partições dos tempos de falha e censura. Usando o critério de Akaike (1974) aconselhado no Capítulo 3 em uma única amostra, foi constatado que o número de intervalos seria $\hat{m}=10$. Entretanto, para esse primeiro processo de estimação, decidimos extrapolar esse número para $m=30$ intervalos para compreender possíveis problemas de toda sorte - computacionalmente quanto consistência dos estimadores.

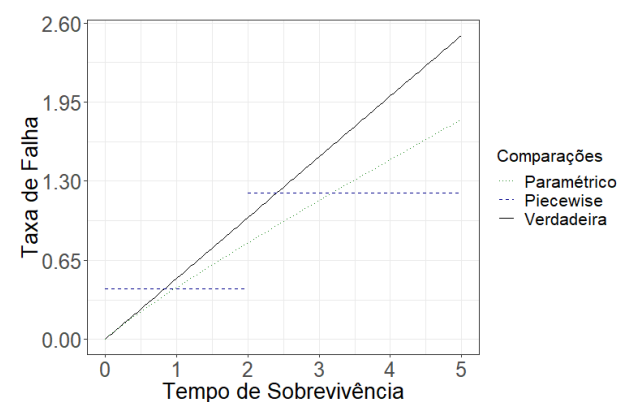

(a) $m_{1}=2$

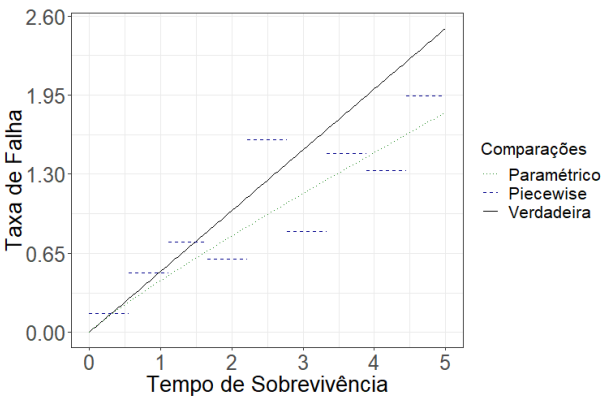

(c) $m_{1}=10$

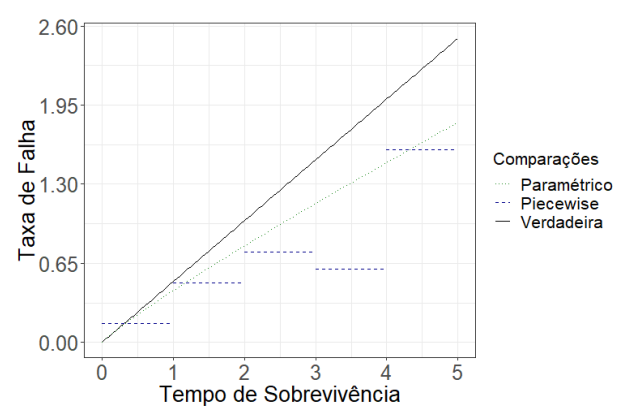

(b) $m_{1}=5$

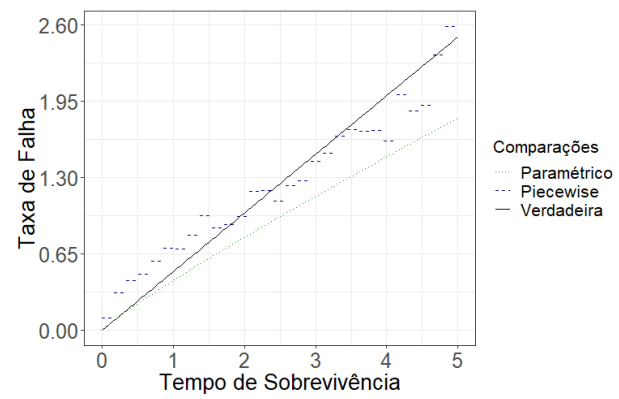

(d) $m_{1}=30$

Figura 4.7: Réplicas de tamanho $n$ para $T$ e $C$ fracamente dependentes

Na Figura 4.7 é apresentado 4 gráficos exemplificando estimativas das funções basais por 
piecewise com número de intervalos $m_{1}$ igual a 2, 5, 10 e 30 para aquela amostra selecionada de um único cenário pelo Esquema I, cópula Frank, $\tau=0,80$ e $n=300$. Note-se com facilidade que, a medida que cresce o valor de $m_{1}$, melhora a estimativa da linha verdadeira. Foi
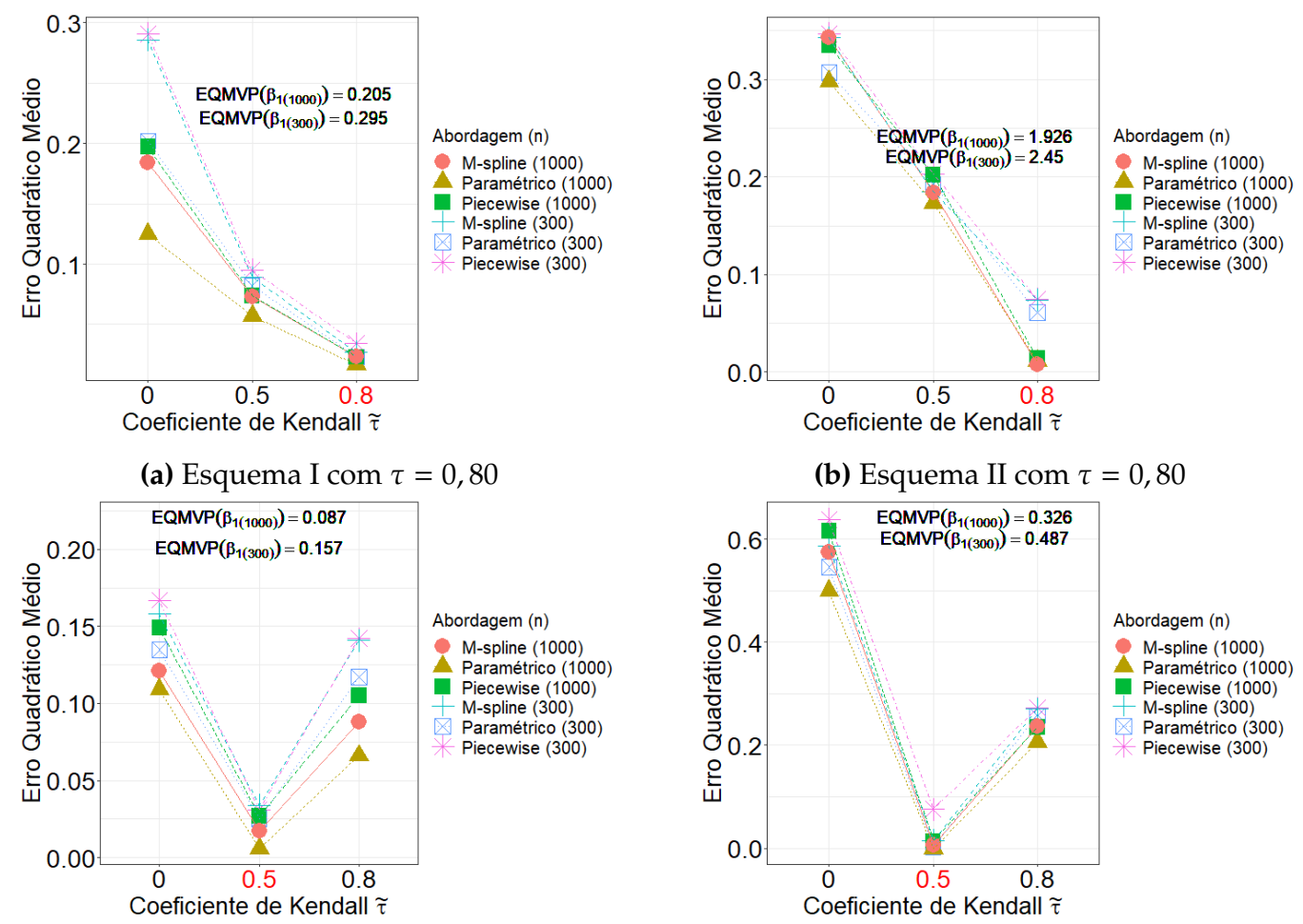

(c) Esquema I $\operatorname{com} \tau=0,50$

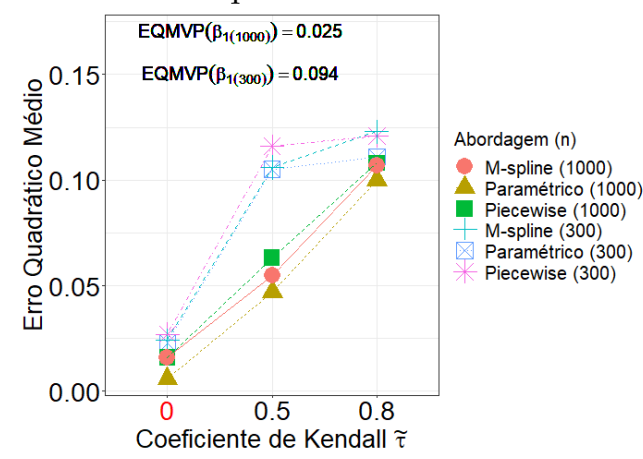

(e) Esquema I $\operatorname{com} \tau=0$

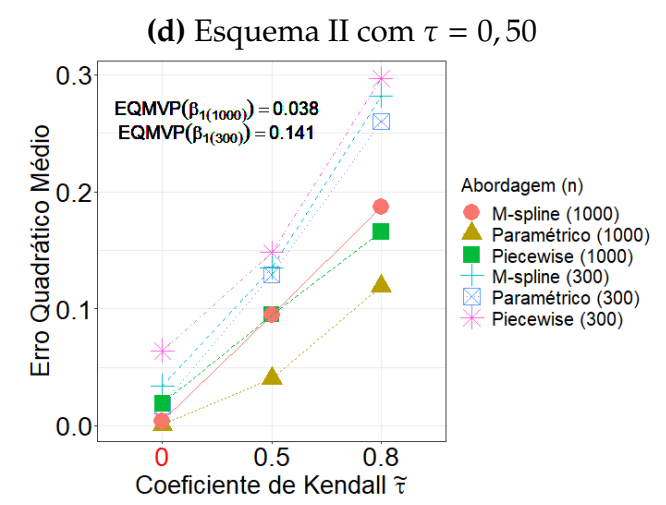

(f) Esquema II $\operatorname{com} \tau=0$

Figura 4.8: Erro quadrático médio para estimativas de $\beta_{1}$, cópula Frank

destacada também a linha estimada pela abordagem paramétrica para enfatizar que a abordagem por partes, embora tenha sempre maior viés, pode ser uma opção segura desde que o número de intervalos minimize o critério. Entretanto, quanto mais se aumenta o número de intervalos, mais se aumenta o custo computacional. Esses eventos são inseparáveis e sempre será necessário lidá-los como em um trade-off para maximizar a performance.

A abordagem paramétrica teve a melhor performance de custo computacional porque, no máximo, tem 4 parâmetros para estimar as funções basais. Entretanto, embora fosse esperado que a abordagem paramétrica também obtivesse a melhor performance no viés e EQM, a 


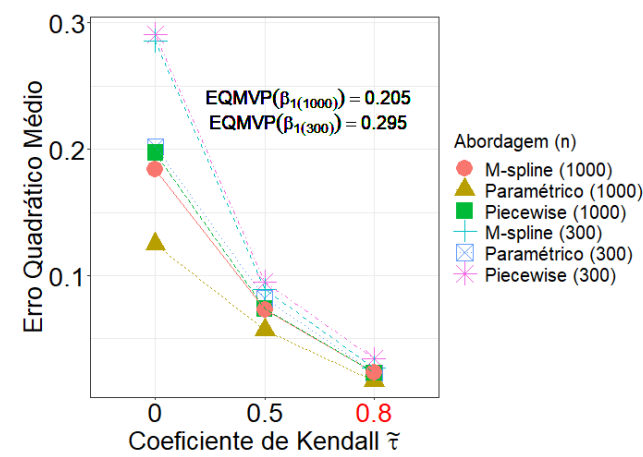

(a) EQM: Esquema I $\operatorname{com} \tau=0,80$

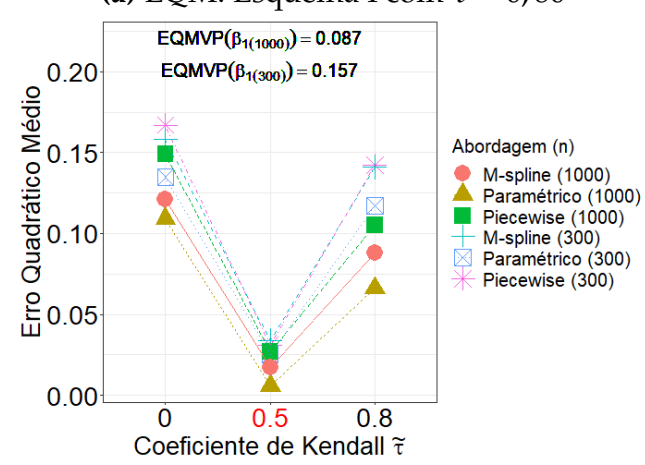

(c) EQM: Esquema I $\operatorname{com} \tau=0,50$

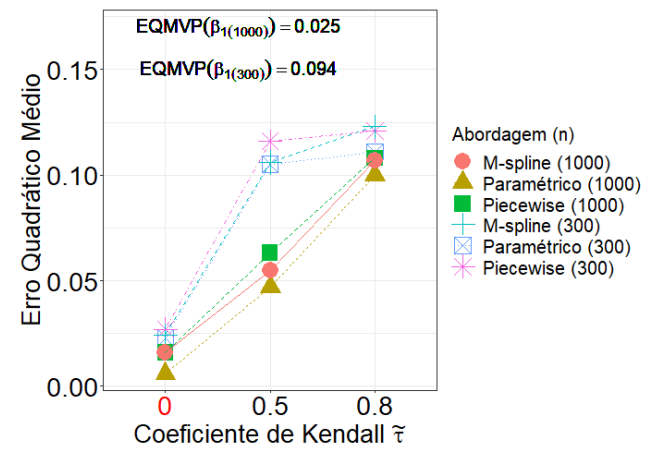

(e) EQM: Esquema I $\operatorname{com} \tau=0$

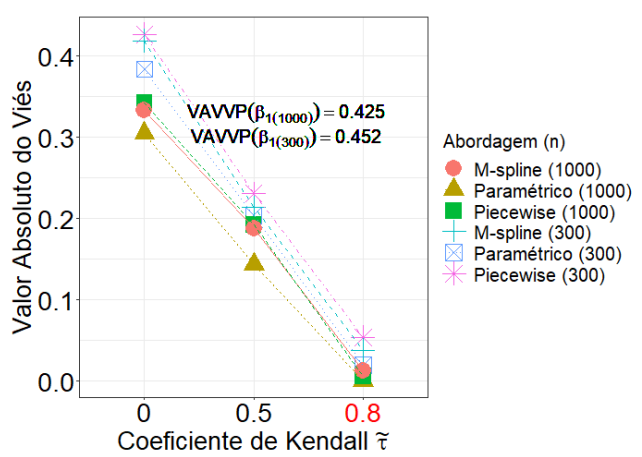

(b) Viés: Esquema I $\operatorname{com} \tau=0,80$

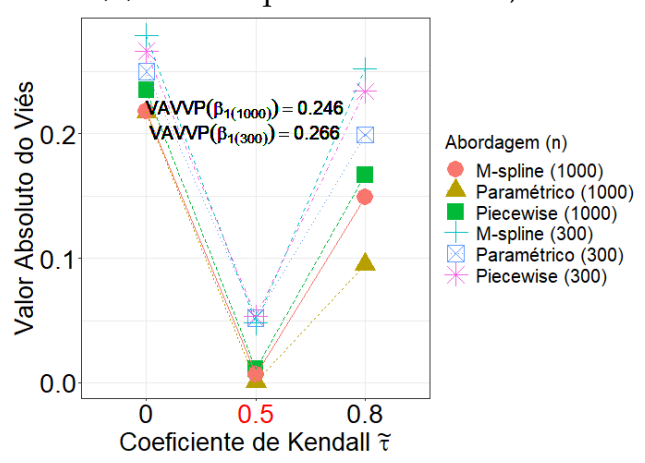

(d) Viés: Esquema I $\operatorname{com} \tau=0,50$

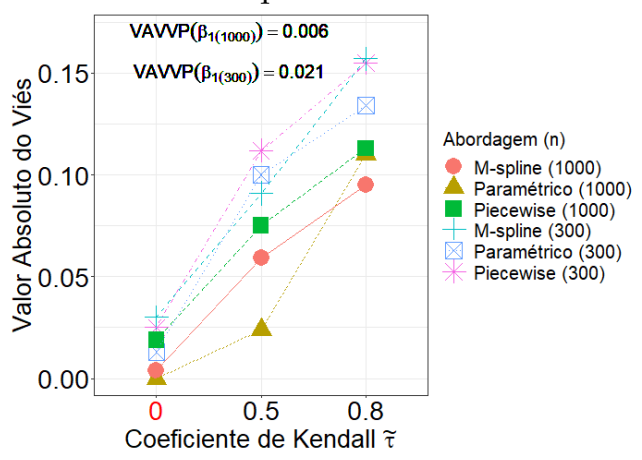

(f) Viés: Esquema I $\operatorname{com} \tau=0$

Figura 4.9: Viés e erro quadrático médio para estimativas de $\beta_{1}$, basais tipo I e cópula Frank

abordagem via $M$-splines obteve melhor performance em alguns cenários pelo viés e erro quadrático médio. Para os processos de estimação paramétrico, a linha de busca foi construída usando a regra de Armijo. Para os processos de estimação semiparamétricos, a linha de busca foi construída usando as condições de Wolfe. Para o leitor ter uma noção na diferença das escolhas dos critérios para linha de busca, enquanto os processos de simulação paramétrica exigiam em média 23 iterações, os processos de estimação via $M$-splines exigiram em média 45 iterações. Já os processos de estimação via piecewise exigiram em média 63 iterações. Portanto, os processos de estimação pela abordagem paramétrica convergiram mais rápido mas com os step-length $\omega_{1}$ e $\omega_{2}$ convergindo a zero muito rápido.

A Figura 4.8 apresenta 6 gráficos a respeito da performance do coeficiente de regressão $\beta_{1}$ sob o aspecto do seu Erro Quadrático Médio (EQM) versus o nível de dependência $\tilde{\tau}$ usados para 
estimação. Os valores de $\tau$ que usamos na geração das amostras estão destacados em vermelho no eixo das abscissas. São gráficos que condensam muita informação sobre as estimativas de $\beta_{1}$ também sob o aspecto do tamanho amostral $n$ e abordagem da aproximação por paramétrico, piecerwise e M-splines. Nos itens 4.8a e 4.8 , nota-se que o EQM das estimativas no Esquema II são superiores ao EQM do Esquema I em quase todas abordagens. Isso se dá naturalmente pela presença de uma alta taxa de dados censurados no Esquema II.

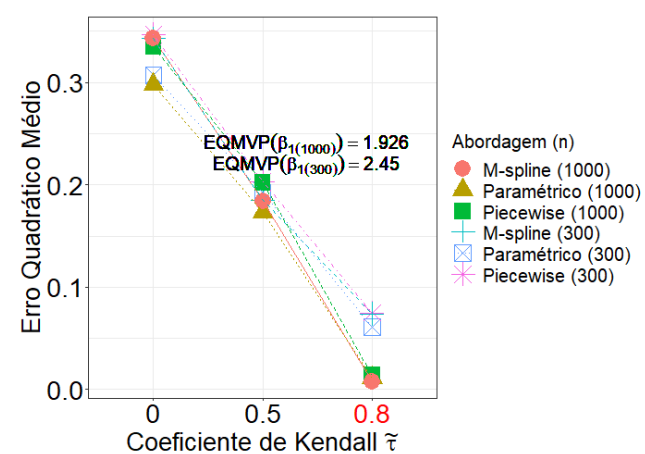

(a) EQM: Esquema I $\operatorname{com} \tau=0,80$

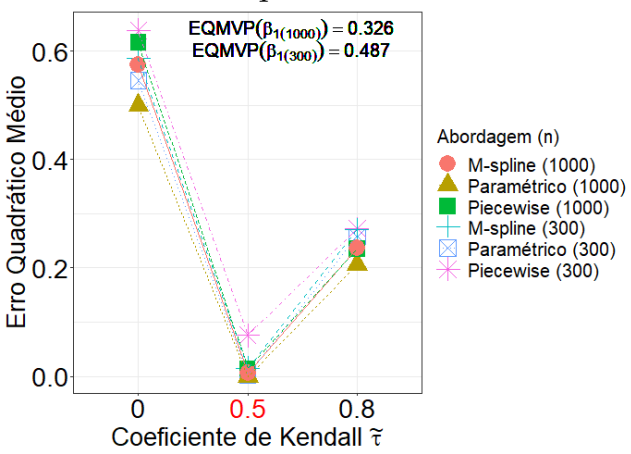

(c) EQM: Esquema II com $\tau=0,50$

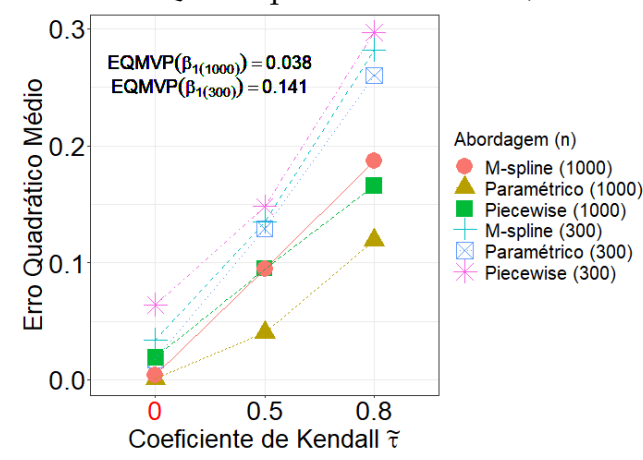

(e) EQM: Esquema II $\operatorname{com} \tau=0$

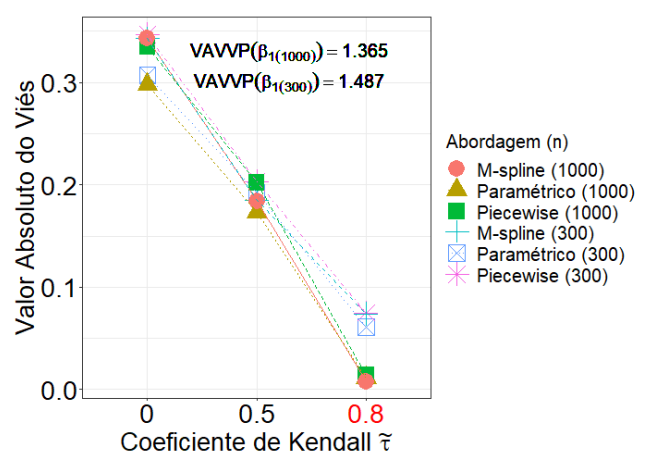

(b) Viés: Esquema II $\operatorname{com} \tau=0,80$

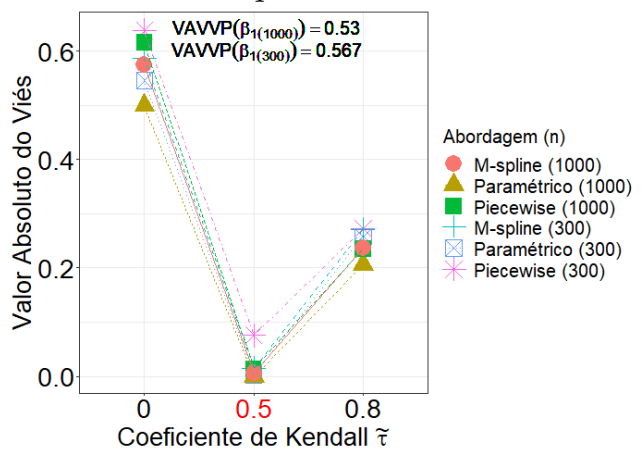

(d) Viés: Esquema II $\operatorname{com} \tau=0,50$

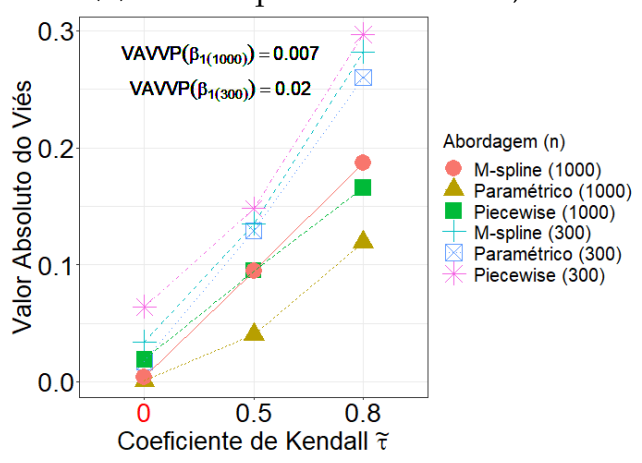

(f) Viés: Esquema II $\operatorname{com} \tau=0$

Figura 4.10: Viés e erro quadrático médio para estimativas de $\beta_{1}$, basais tipo II e cópula Frank

A Figura 4.9 apresenta 6 gráficos paralelizando o EQM com o valor absoluto do viés do parâmetro $\beta_{1}$, sendo um resumo das estimativas obtidas na performance das amostras geradas pelo Esquema I. Já a Figura 4.10 apresenta também 6 gráficos com resumo das estimativas mas agora pelas amostras geradas pelo Esquema II. De fato, a abordagem via M-splines em geral obteve melhor performance quando comparada pela abordagem piecewise. 


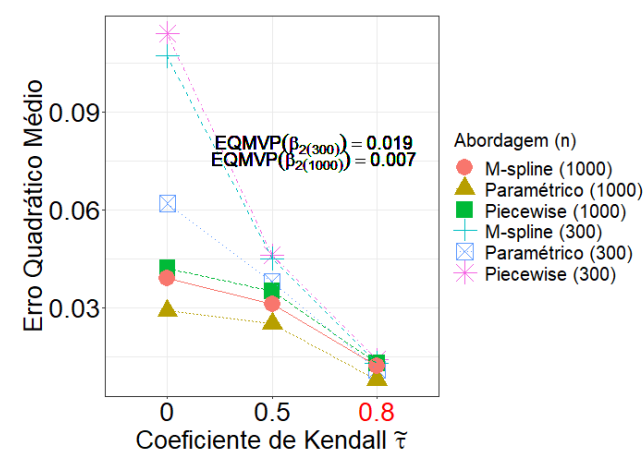

(a) Esquema I $\operatorname{com} \tau=0,80$

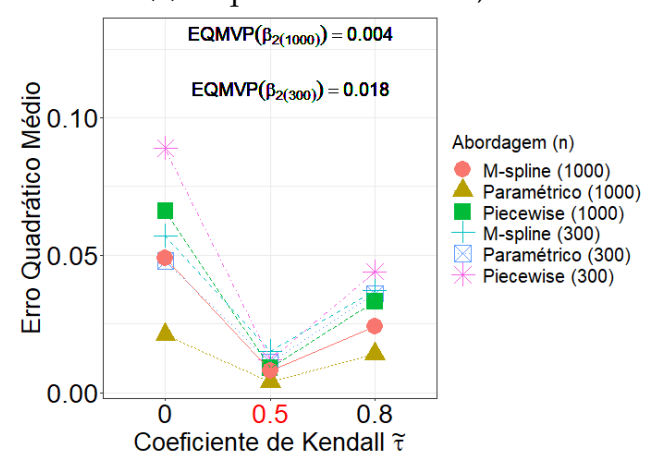

(c) Esquema I $\operatorname{com} \tau=0,50$

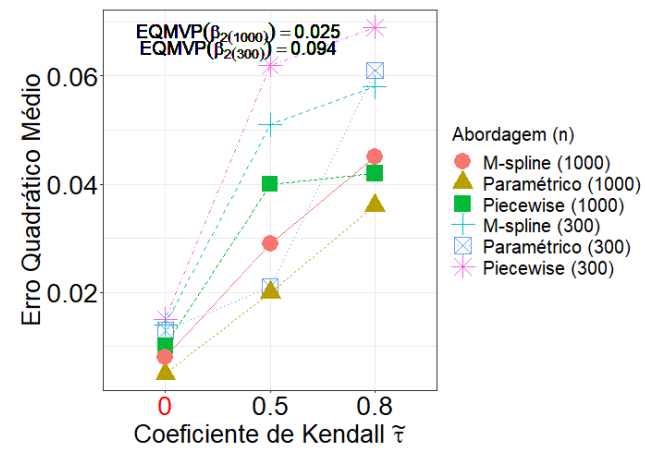

(e) Esquema I $\operatorname{com} \tau=0$

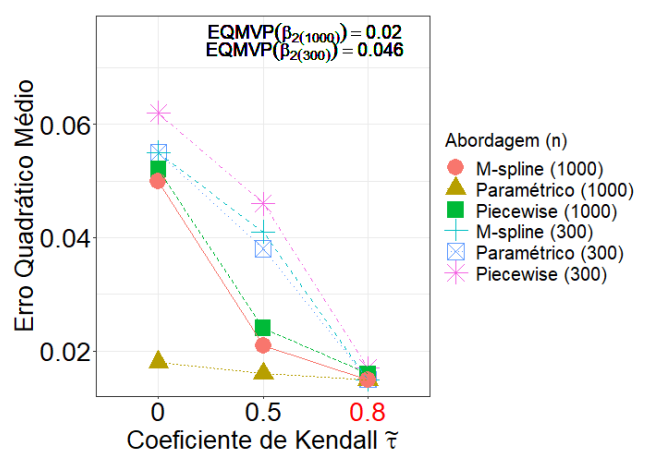

(b) Esquema II $\operatorname{com} \tau=0,80$

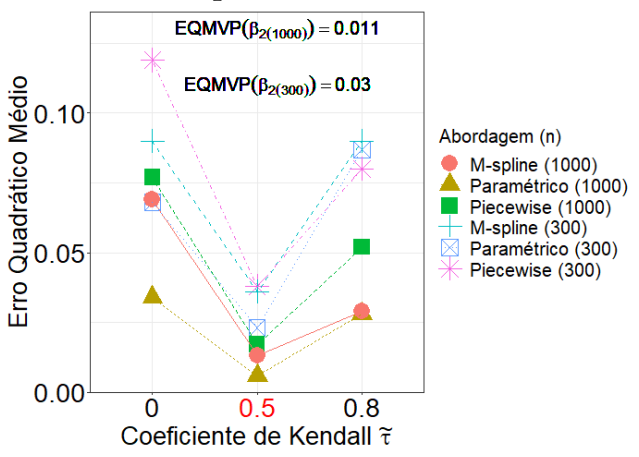

(d) Esquema II $\operatorname{com} \tau=0,50$

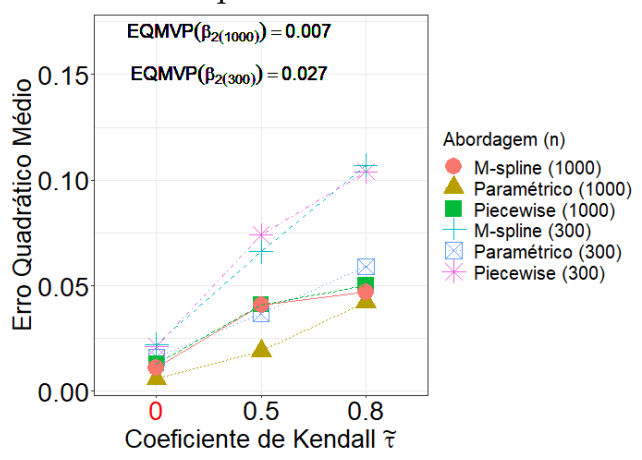

(f) Esquema II $\operatorname{com} \tau=0$

Figura 4.11: Erro quadrático médio para $\beta_{2}$, cópula Frank

Todas figuras anteriores (4.8-4.10) bem como as seguintes possuem valores denotados por $\operatorname{EQMVP}\left(\beta_{j(n)}\right)$. Esses valores significam o erro quadrático médio de $\beta_{j}$ calculado a partir dos processos de estimação usando a função de log-verossimilhança parcial. Portanto, $\operatorname{EQMVP}\left(\beta_{j(300)}\right)$ significa o EQMVP para amostras de tamanho $n=300$ e $\operatorname{EQMVP}\left(\beta_{j(1000)}\right)$ significa o EQMVP para amostras de tamanho $n=1000$ - amostras geradas pela cópula de Frank com nível de dependência $\tau$ destacado em vermelho no eixo das abscissas.

A Figura 4.11 apresenta 6 gráficos agora para o parâmetro $\beta_{2}$. Talvez a primeira observação seja que o EQMVP agora disputa melhor desempenho com o EQM da função de verossimilhança. No item (a) da Figura 4.11, por exemplo, o EQM para as réplicas de tamanho $n=1000$ ficam em torno de 0,150 enquanto o EQMVP é igual a 0,007. O porquê desse fenômeno pode ser explicado mediante a paralelização fornecida nas figuras 4.12 e 4.13. Os valores absolutos dos 


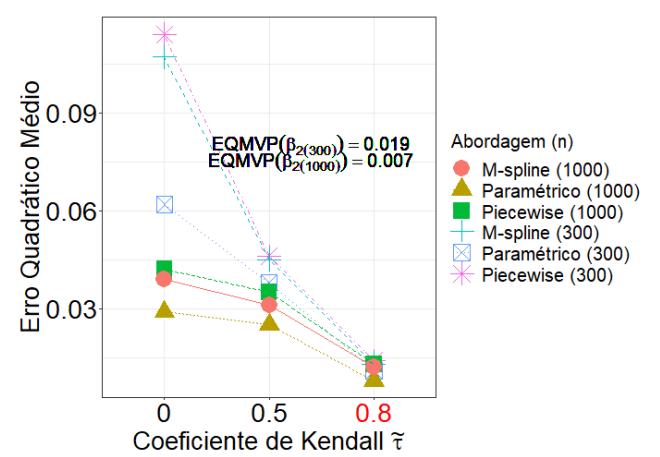

(a) EQM: Esquema I $\operatorname{com} \tau=0,80$

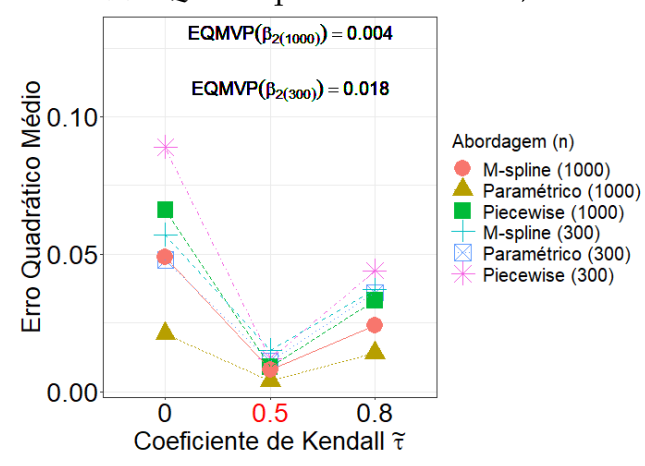

(c) EQM: Esquema I $\operatorname{com} \tau=0,50$

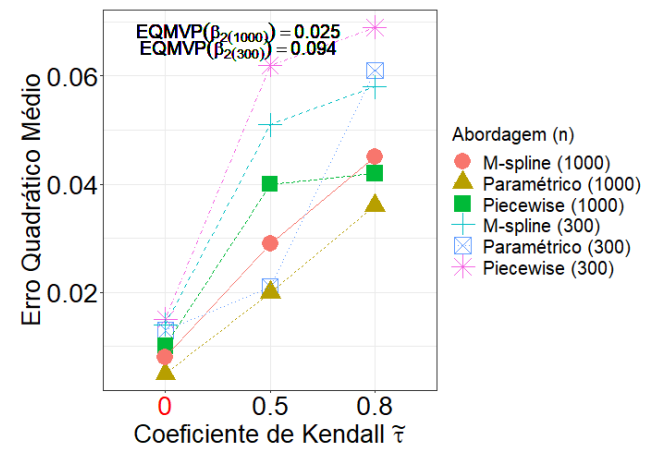

(e) EQM: Esquema I $\operatorname{com} \tau=0$

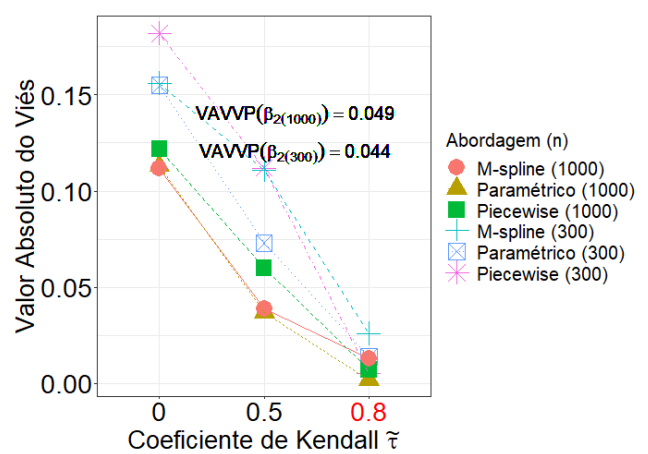

(b) Viés: Esquema I $\operatorname{com} \tau=0,80$

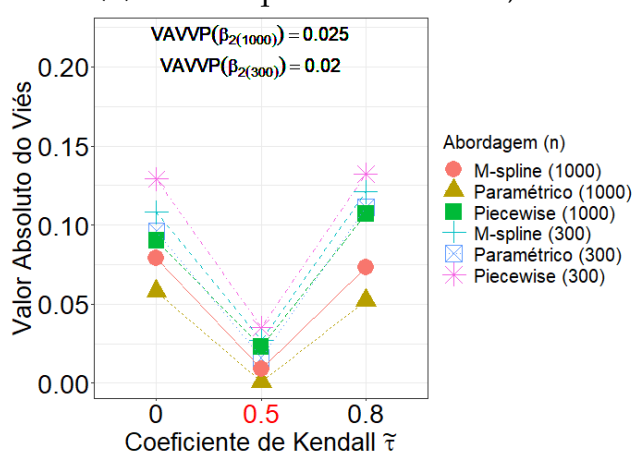

(d) Viés: Esquema I $\operatorname{com} \tau=0,50$

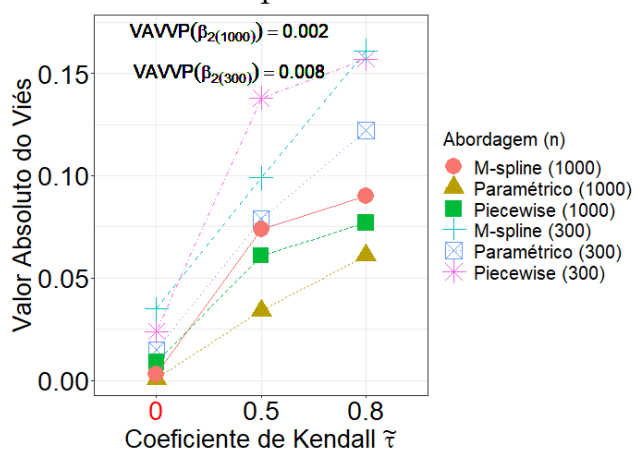

(f) Viés: Esquema I com $\tau=0$

Figura 4.12: Viés e erro quadrático médio para $\beta_{2}$, basais tipo I e cópula Frank

vieses são mais competitivos entre si, o que implica em um maior erro padrão nas estimativas usando a verossimilhança de $\mathrm{Xu}$ et al. (2018).

É preciso distinguir as performances usando as funções de verossimilhança de $\mathrm{Xu}$ et al. (2018) e Huang e Zhang (2008), porque a primeira segue o esquema na Figura 4.6 e a segunda foi realizada com o mesmo número $N=1300$ de réplicas mas o tamanho amostral de $n=500$ e com somente 4 cenários. O motivo para essa redução drástica de cenários deve-se ao custo computacional exigido pela função de verossimilhança. Esse tópico será apresentado mais adiante.

A Tabela 4.2 apresenta todos os resultados das estimativas obtidas em 4 cenários para o modelo de Huang e Zhang (2008). Na primeira coluna são apresentadas algumas características presentes nas réplicas como o tipo de esquema e a proporção média de censura dependente 


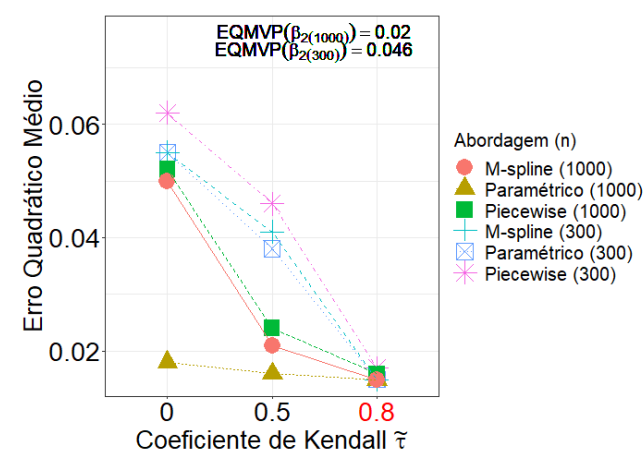

(a) EQM: Esquema II com $\tau=0,80$

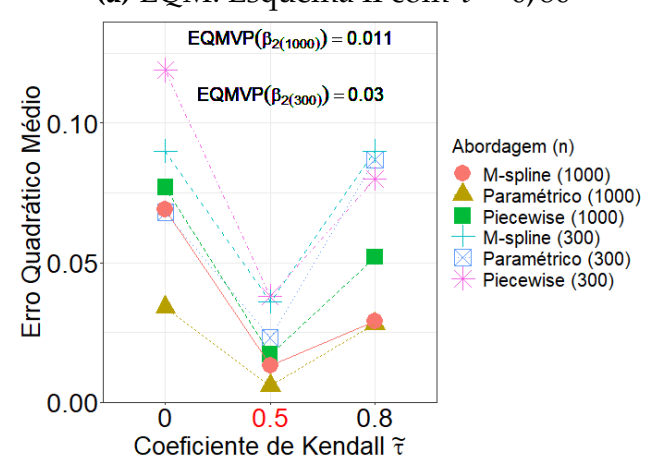

(c) EQM: Esquema II $\operatorname{com} \tau=0,50$

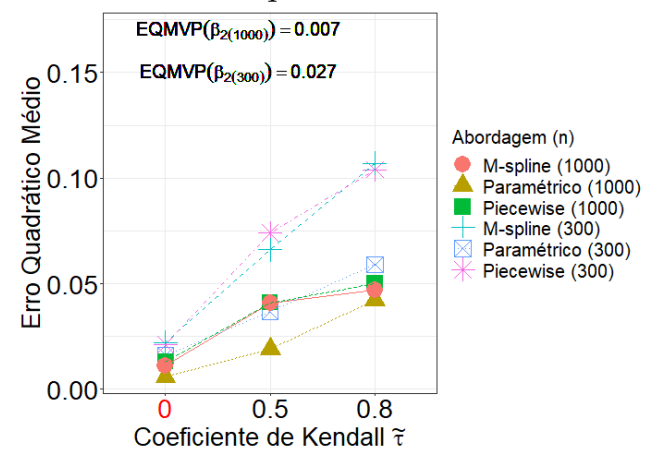

(e) EQM: Esquema II $\operatorname{com} \tau=0$

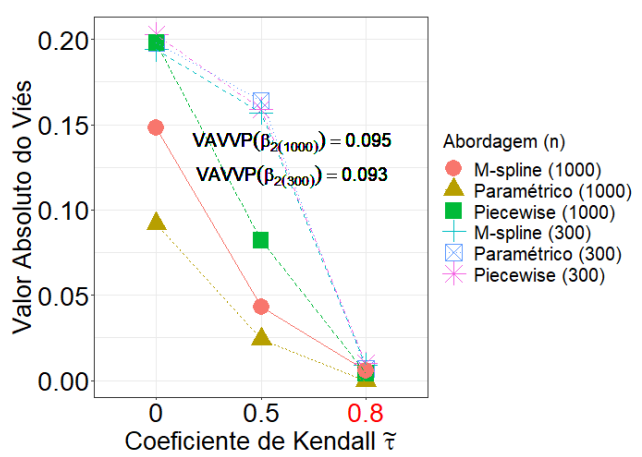

(b) Viés: Esquema II $\operatorname{com} \tau=0,80$

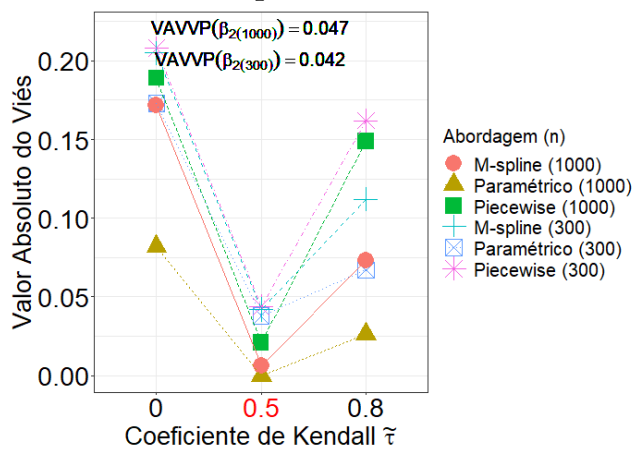

(d) Viés: Esquema II $\operatorname{com} \tau=0,50$

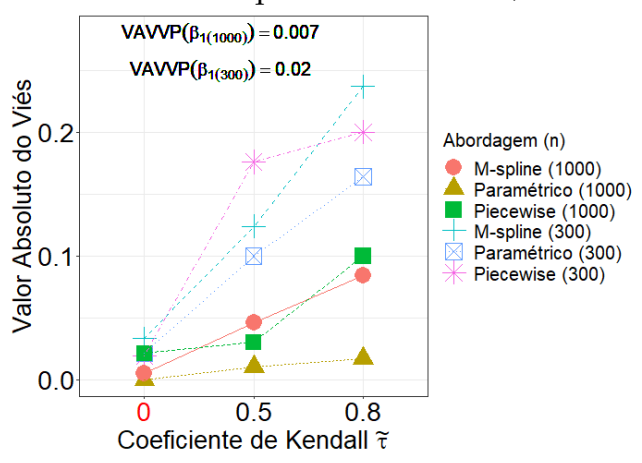

(f) Viés: Esquema II $\operatorname{com} \tau=0$

Figura 4.13: Viés e erro quadrático médio para $\beta_{2}$, basais tipo II e Cópula Frank

$\bar{\pi}_{C}$ em dois níveis: como moderada o que é aqui $35,6 \%$ e elevada com mais de $60 \%$. No geral, o valor absoluto do viés não é tão competitivo contra a performance da verossimilhança de $\mathrm{Xu}$ et al. (2018). Assim, sugerindo que a suavização nas abordagens por aproximação linear das funções basais podem ter surtido uma performance com menos viés . Não é conclusivo. É preciso levar em consideração que o tamanho amostral foi fixado em $n=500$.

Voltando sobre a Figura (4.14), notamos que, entre as duas abordagens semiparamétricas, a curva mais próxima da verdadeira é pela abordagem via $M$-splines. Ademais, pelos resultados apresentados em Xu et al. (2018), já era esperado que a abordagem via piecewise aumentaria o viés da estimação da curva ao longo do tempo de sobrevivência.

A Figura 4.15 apresenta 6 gráficos das curvas estimadas das funções basais para o tempo de falha. Observe que a curva estimada se afasta mais da verdadeira nos gráficos feitos pelo 


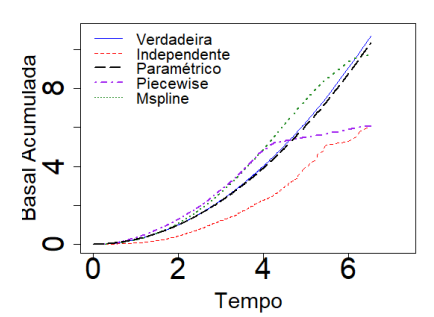

(a) $n=300$ e $\tau=0,80$

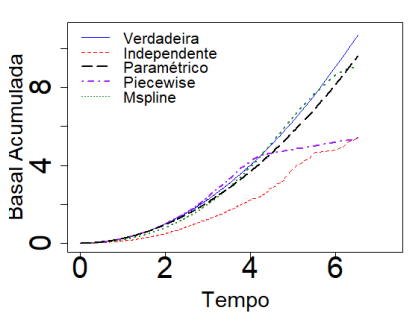

(d) $n=1000$ e $\tau=0,50$

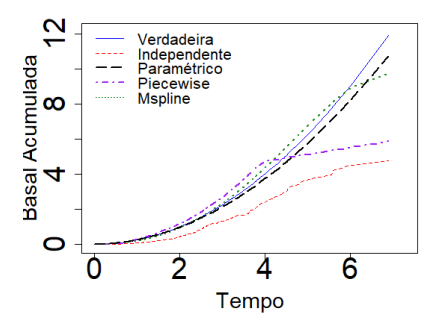

(b) $n=1000$ e $\tau=0,80$

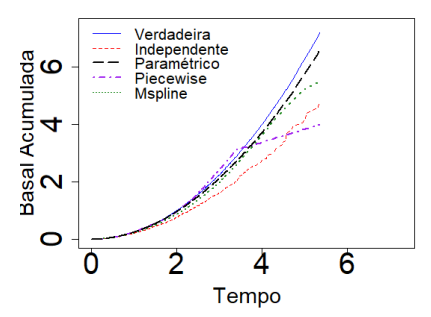

(e) $n=300$ e $\tau=0$

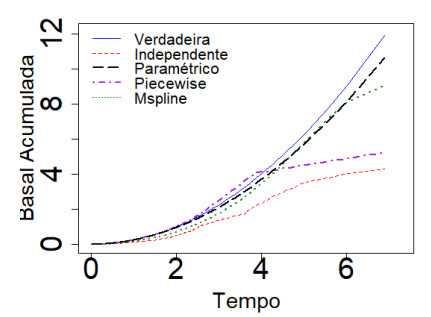

(c) $n=300$ e $\tau=0,50$

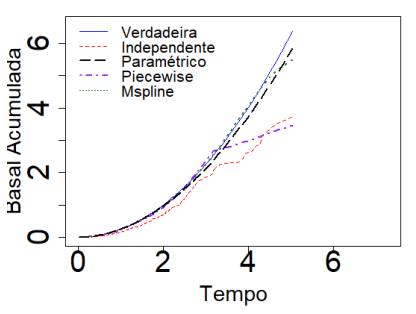

(f) $n=1000$ e $\tau=0$

Figura 4.14: Curvas das Funções Basais Acumuladas para Esquema I, Frank e $\tilde{\tau}=\tau$

Esquema II.

Pela modelagem semiparamétrica, Xu et al. (2018) adicionaram uma penalidade na função de verossimilhança para suavizar o método de aproximação das funções basais do tempo de falha e censura. Quanto aos coeficientes de suavização $h_{1}$ e $h_{2}=0$ para a função de verossimilhança de $\mathrm{Xu}$ et al. (2018), foi realizado uma validação cruzada com 10-fold em uma única amostra e ficou obtido que a minimização de $L C V_{1}$ atinge o valor de $h_{1}=0,02$. Entretanto, a medida que se aproxima $h$ de zero, ficou constatado que minimiza mais ainda o $L C V_{1}$. Foi provado por Xu et al. (2018) que a normalidade de $\eta$ se dá quando $h_{1} \longrightarrow 0$ e $h_{2} \longrightarrow 0$.

Voltando a comparar as verossimilhanças aqui utilizadas, este trabalho corrobora com os autores $\mathrm{Xu}$ et al. (2018) quanto ao ganho de qualidade nas aproximações das funções basais. Entretanto, é preciso ressaltar que, ao que tudo indica, a abordagem via M-splines é mais interessante que as demais. A abordagem paramétrica é limitada e não há no momento como testar se os tempos de falha seguem a distribuição de Weibull. A abordagem por piecewise não tem essa limitação, mas o custo computacional em função de $m$ foi um desafio no estudo de simulação. Foram meses de refinamento no algoritmo para finalmente obter uma performance razoável. No fim, os resultados ainda apresentaram um erro quadrático médio quase sempre superior comparando com M-splines. Por outro lado, a verossimilhança de Huang e Zhang (2008) foi também desafiadora tanto pelo custo computacional quanto pelos complicados resultados analíticos do vetor gradiente e matriz hessiana. 
4.2 Tempo de Sobrevivência Ajustada pela Qualidade de vida: uma análise de sensibilidade exploratória

Tabela 4.2: Estimativas das réplicas usando a função de verossimilhança Huang e Zhang (2008)

\begin{tabular}{|c|c|c|c|c|c|c|c|c|c|c|c|c|c|c|c|c|}
\hline \multicolumn{17}{|c|}{ Resumo da Performance pelas Estimativas em $N=500$ réplicas de tamanho $n=500$} \\
\hline & & \multicolumn{5}{|c|}{$\tau=0,80$} & \multicolumn{5}{|c|}{$\tau=0,50$} & \multicolumn{5}{|c|}{$\tau=0$} \\
\hline & & $B\left(\eta_{j}\right)$ & $\hat{\theta}_{M C}\left(\eta_{j}\right)$ & $\hat{\theta}_{A S}\left(\eta_{j}\right)$ & EQM & $\mathrm{CP}(\%)$ & $B\left(\eta_{j}\right)$ & $\hat{\theta}_{M C}\left(\eta_{j}\right)$ & $\hat{\theta}_{A S}\left(\eta_{j}\right)$ & EQM & $\mathrm{CP}(\%)$ & $B\left(\eta_{j}\right)$ & $\hat{\theta}_{M C}\left(\eta_{j}\right)$ & $\hat{\theta}_{A S}\left(\eta_{j}\right)$ & EQM & $\mathrm{CP}(\%)$ \\
\hline Esque & $\beta_{2}$ & 0 & 90 & 0,101 & 0,012 & 08 & 0,013 & 15 & 0,130 & 0,013 & 97,08 & 0,021 & 111 & 0,125 & 0,013 & 96,32 \\
\hline Cópula & $\phi_{1}$ & 0,049 & 56 & 0,167 & 0,027 & 31 & $-0,009$ & 0,142 & 0,161 & 0,020 & 97,15 & & 0,139 & 0,154 & 0,021 & 95,92 \\
\hline$\tilde{\tau}=\tau$ & $\phi_{2}$ & 0,034 & 0,104 & 0,123 & 0,012 & 96,62 & 0,052 & 0,110 & 0,124 & 0,015 & 95,54 & 0,052 & 0,107 & 0,118 & 0,014 & 94,54 \\
\hline Cópula & $\phi_{1}$ & 0,035 & 105 & 0,119 & 0,012 & 96,08 & 0,006 & & 0,119 & 0,011 & 96,31 & 0,024 & 0 & 0,154 & 0,017 & 96,00 \\
\hline$\tilde{\tau}=\tau$ & $\phi_{2}$ & 0,017 & 0,088 & 0,107 & 0,008 & 97,31 & 0,014 & 0,083 & 0,093 & 0,007 & 96,38 & 0,010 & 0,098 & 0,118 & 0,010 & 95,54 \\
\hline $\bar{\pi}_{C}$ & $\beta_{1}$ & 0,015 & 19 & 65 & 0,022 & $=$ & 0,031 & 0,099 & 0,109 & 1 & 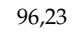 & 7 & 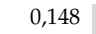 & 162 & 31 & 93,62 \\
\hline Esqu & $\beta_{2}$ & 0 & 15 & 29 & 0,015 & & $-0,049$ & & 0,110 & 0, & & 32 & & 128 & 0,014 & 96,62 \\
\hline Copula Clayton & $\phi_{1}$ & 0,047 & 143 & 0,159 & 0,023 & 96,15 & 0,000 & 0,145 & 0,159 & 0,021 & 96,38 & 0,046 & 1 & 0,159 & 0,022 & 96,31 \\
\hline$\tilde{\tau}=\tau$ & $\phi_{2}$ & 0,052 & 0,110 & 0,121 & 0,015 & 94,46 & 0,059 & 0,111 & 0,123 & 0,016 & 94,23 & 0,053 & 0,109 & 0,121 & 0,015 & 94,69 \\
\hline
\end{tabular}

\subsection{Tempo de Sobrevivência Ajustada pela Qualidade de vida: uma análise de sensibilidade exploratória}

Esse estudo de simulação foi realizado para avaliar a aplicação de modelos sob censura dependente baseados em cópulas para a análise do tempo de sobrevivência ajustado pela qualidade de vida, que é a principal motivação deste trabalho.

O TSAQV deve ser calculado com base no escore QV a partir do ponto inicial do acompanhamento de $n$ pacientes. No estudo de simulação presente, ficou decidido usar 4 períodos equidistantes para obter o QV: $t_{1}=0, t_{2}=1, t_{3}=2$ e $t_{4}=3$. Tempos acima do período $W=4$ foram censurados, porque ficou considerado que $W$ é o limite superior do hipotético acompanhamento. Nesse exercício de simulação está sendo levado em consideração. Portanto, o TSAQV do $i$-ésimo paciente será escrito como

$$
U_{i}^{o b s}= \begin{cases}Q V_{i 0} t_{i 0}+Q V_{i 1} t_{i 1}+Q V_{i 2} t_{i 2}+Q V_{i 3}\left(t_{i 3}-W\right), & \text { se } T_{i}>4 \\ Q V_{i 0} t_{i 0}+Q V_{i 1} t_{i 1}+Q V_{i 2} t_{i 2}+Q V_{i 3} t_{i 3}, & \text { caso contrário. }\end{cases}
$$

A equação 4.1) é escrita com o escore $Q V_{i j}$ do $i$-ésimo paciente obtido na $j$-ésima avaliação em que $j=0$ é o momento da admissão do paciente. Ainda sobre a última equação, o coeficiente $t_{i j}$ é o tempo do $i$-ésimo paciente viveu sob a j-ésima qualidade de vida. Caso o paciente tenha tempo de sobrevida maior que 4 , seu tempo de vida sob a última qualidade de vida sofrerá uma redução de $t_{i 3}-W$. Por exemplo, imagine que o $k$-ésimo paciente tenha o tempo de sobrevivência igual a $t_{i}=1,5$. Dado os 4 períodos equidistantes do $Q V, t_{k 0}=1$ e $t_{k 1}=0,5$. Portanto, o TSAQV do $k$-ésimo paciente é

$$
U_{k}^{o b s}=Q V_{k 0}+0,5 Q V_{k 1}
$$

Outro exemplo é quando um paciente teve um tempo de sobrevida igual a 5,57 então o TSAQV será igual a $U^{o b s}=Q V_{0}+Q V_{1}+Q V_{2}+1,57 Q V_{3}$, pois os tempos que o paciente vive sob 


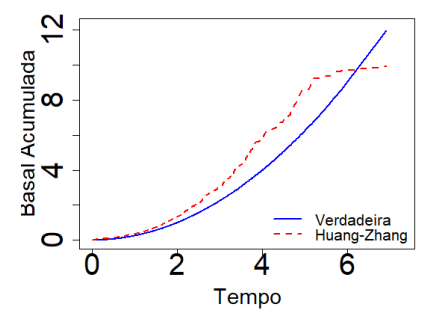

(a) Esquema I e $\tau=0,80$

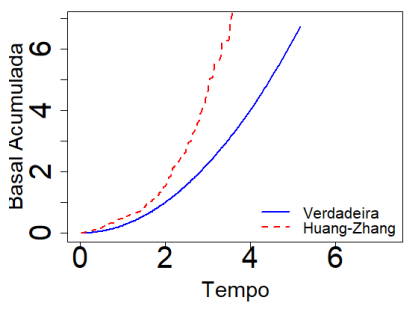

(d) Esquema II e $\tau=0,50$

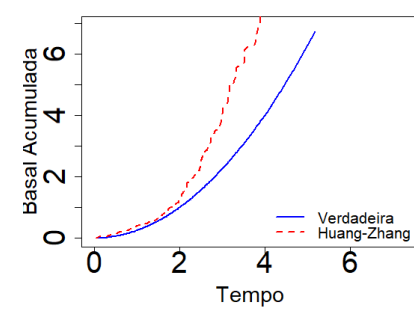

(b) Esquema II e $\tau=0,80$

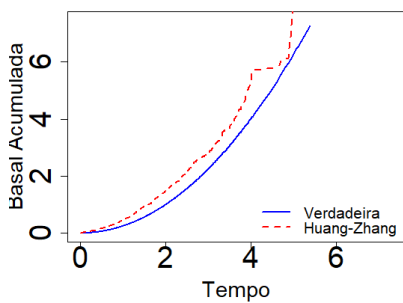

(e) Esquema I e $\tau=0$

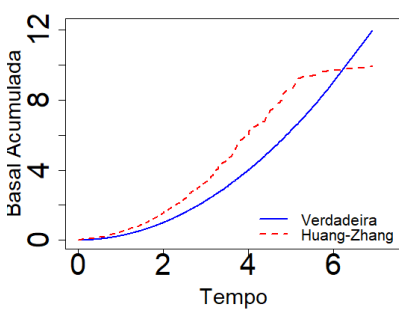

(c) Esquema I e $\tau=0,50$

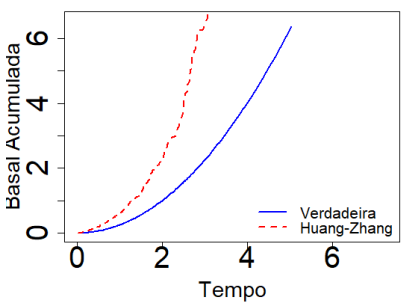

(f) Esquema II e $\tau=0$

Figura 4.15: Curvas das funções basais estimadas da verossimilhança de Huang e Zhang (2008)

qualidade de vida são equidistantes excetuando seu tempo de sobrevida na última qualidade de vida. Os escores QV são números aleatórios entre 0 e 1, então, supondo que o paciente teve todos escores iguais a 1, o TSAQV o tempo de falha ou censura.

A seguir, uma lista como um passo a passo para gerar uma amostra do tempo de sobrevivência ajustado pelos escores de qualidade de vida a um paciente

(M1) gere a variável preditora $Z_{1 i} \sim \operatorname{Bernoulli}(0,5)$ e $Z_{2 i} \sim \operatorname{Normal}(0,1)$;

(M2) gere o tempo de falha $T_{i} \sim \operatorname{Weibull}\left(2, \frac{4}{\exp \left(\mathbf{Z}_{i}^{\top} \boldsymbol{\beta}\right)}\right)$ independente de $C_{i}$;

(M3) gere os escores $Q V_{i j}$ seguindo alguma distribuição contínua inflacionada de 1 em função de $\beta$ e $Z_{i}^{\top}$, mas cada escore dependente do outro por meio de uma cópula com nível de dependência fraco $\tau$;

(M4) para cada indivíduo $i$, faça a combinação linear em (4.1) para gerar o Tempo de Falha Ajustado pela Qualidade de Vida (TFAQV).

Os pontos (M2) e (M3) certamente são os mais delicados e requerem mais detalhes. Diferentemente da seção anterior, o tempo de falha é gerado independentemente do tempo de censura para cada paciente. Sendo assim, o ponto (M3) segue a formulação em Gelber et al. (1989) de que a introdução dos escores é que gera dependência entre os tempos de falha e tempos de censura. A formulação para gerar o Tempo de Censura Ajustado pela Qualidade de Vida (TCAQV) é semelhante a lista anterior, excetuando que $C_{i} \sim \operatorname{Exponencial}\left(\frac{1}{5} \exp \left(Z_{i}^{\top} \phi\right)\right)$. Então o TSAQV para cada paciente é igual ao mínimo entre TFAQV e TCAQV.

Foi decidido usar a distribuição Beta Inflacionada de 1 proposta em Ospina e Ferrari (2010) para gerar os escores dependentes. Existe uma rotina para gerar os valores no pa- 
cote gamlss: : BEINF 1. Os valores dos parâmetros da simulação foram fixados em $\sigma_{i}=v_{i}=0,5$ e a média $\mu_{i}$ para cada paciente como função ligação

$$
\mu_{i}=\frac{\exp \left(\boldsymbol{Z}_{i}^{\top} \boldsymbol{\beta}\right)}{1+\exp \left(\boldsymbol{Z}_{i}^{\top} \boldsymbol{\beta}\right)}
$$

Para gerar uma amostra usando o pacote copula: : rMvdc disponível no R, foi decido usar $\tau=0,20$ para obter os escores fracamente dependentes.

A geração de dados seguindo o esquema proposto é simples, porém a dificuldade que surge é que não se conhece a distribuição do TSAQV resultante. Além disso, não é possível saber se os modelos considerados são adequados para ajustar o TSAQV e, caso sejam, não se sabe os valores dos parâmetros associados. Para contornar esse problema, gerou-se uma amostra muito grande ( $n=100000$ ) e foi realizado um estudo para avaliar a adequabilidade do uso do modelo de taxas de falha proporcionais. Considerando os dados sem censura, foi ajustado um modelo de Cox e feita a avaliação dos resíduos. Essa análise indicou que um modelo de taxas de falha proporcionais é adequado. Além disso, os valores estimados dos parâmetros foram tomados como referência para comparação dos métodos de estimação na presença de censura dependente. Portanto, o propósito é verificar se é vantajoso ou não adotar a premissa de dependência na censura no TSAQV usando a verossimilhança de Xu et al. (2018) em contraste da verossimilhança parcial (usando a premissa de independência).

\subsubsection{Resultados}

Na presente subseção serão apresentados os principais resultados do estudo de simulação exploratória para o TSAQV. Assim como na seção anterior, os critérios da performance são o viés $B\left(\eta_{j}\right)$, a média do erro padrão via Monte Carlos $\overline{\hat{\sigma}}_{M C}$, a média do erro padrão assintótico $\overline{\hat{\sigma}}_{A S}$, o Erro Quadrático Médio (EQM) e uma probabilidade de cobertura (CP) de $96 \%$.

Diferentemente da seção anterior, os cenários aqui são apenas dois diferenciando somente o tamanho amostral em $n=300$ e $n=1000$. Mesmo assim, foi preferido usar gráficos para explicar os resultados pois o nível de dependência $\tilde{\tau}$ variou em 9 valores. Foram geradas $N=1300$ réplicas novamente e todas foram estimadas pela função de verossimilhança de $\mathrm{Xu}$ et al. (2018). Nesse estudo não foi considerado usar a verossimilhança de Huang e Zhang (2008).

Em um primeiro momento, ficou o questionamento de qual seria o verdadeiro valor de $\tau$ quando se simulada o TSAQV. Para respondê-lo, foi pego ambas amostras de tamanho $n=100000$ tanto do TFAQV e TCAQV e foi utilizado a rotina copula : : fi tCopula para obter a estimativa de $\tau$. Felizmente, pela conveniência, o resultado da estimativa ficou muito próximo do valor administrado nas simulações separadas, ou seja, $\hat{\tau} \approx 0,20$. Também foi adotado simular $N=5000$ réplicas de tamanho $n=300$ e obter para cada réplica a estimativa $\tilde{\tau}$. A média $\overline{\tilde{\tau}}$ também ficou próxima a 0,20 com erro padrão via Monte Carlos inferior a 0,05.

Destaca-se que não foi considerado usar a abordagem paramétrica porque não foram encontradas evidências que o TSAQV segue a mesma distribuição que o Tempo de Sobrevivência. 


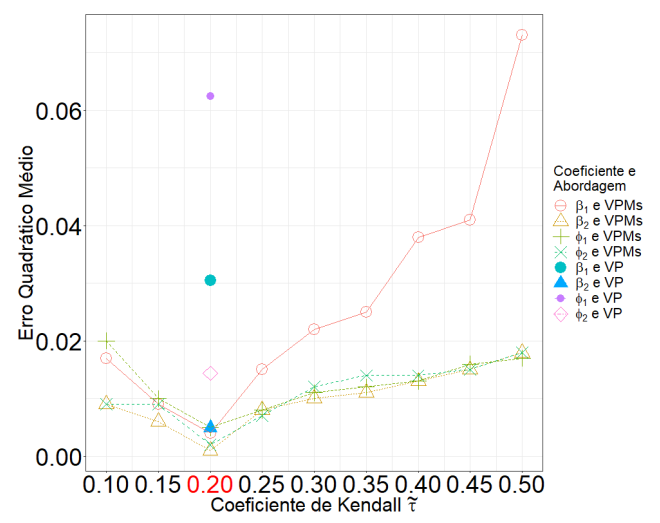

(a) $n=300$

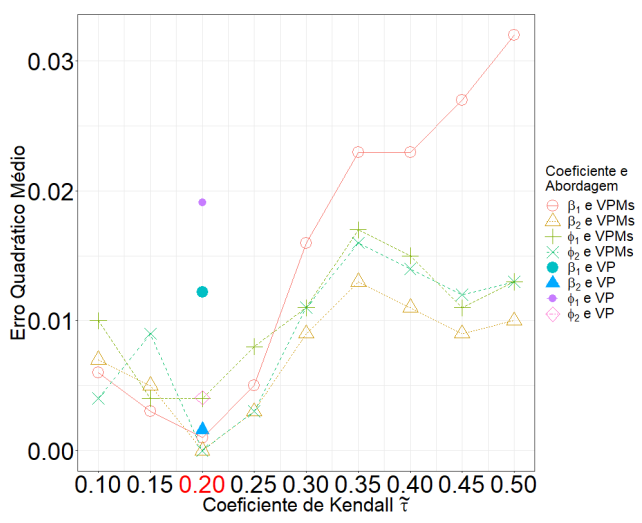

(b) $n=1000$

Figura 4.16: EQM das Estimativas para TSAQV Cópula Frank e via $M-$ splines

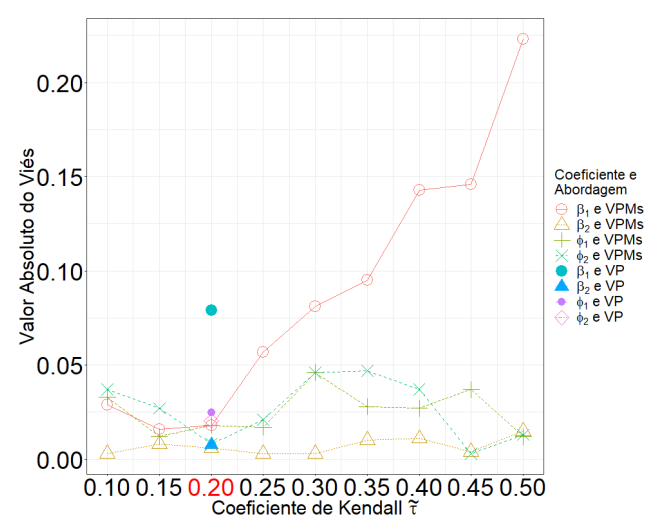

(a) $n=300$

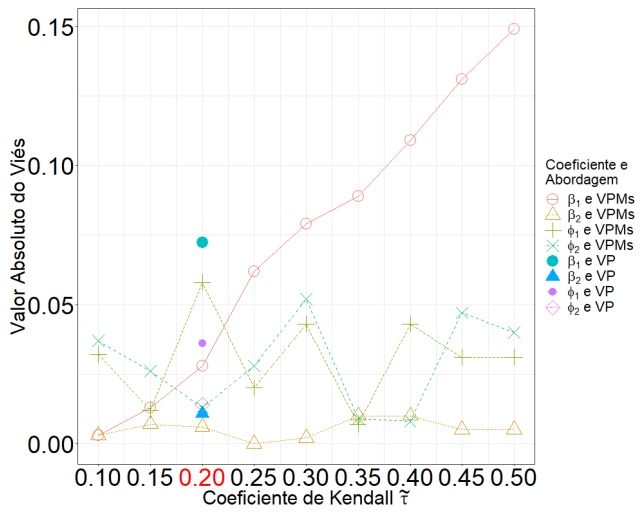

(b) $n=1000$

Figura 4.17: Valor Absoluto do Viés das Estimativas para TSAQV Cópula Frank e via $M$ - splines

Na Figura 4.14 são apresentados 2 gráficos mostrando o EQM para cada estimativa variando o $\tilde{\tau}$. Talvez a primeira observação seja a diferença de amplitude naturalmente causada pelo tamanho amostral. Na legenda, a sigla VP significa verossimilhança parcial e VPMs significa verossimilhança via $M$-splines. Na prática, obter o $\tau$ em TSAQV não é possível. Então ficou decidido entender o quanto poderia ficar ruim a performance na vizinhança do valor verdadeiro aumentando a amplitude de $\tilde{\tau}$. A Figura 4.15 apresenta dois gráficos sobre o valor absoluto do viés. Por ela, é possível chegar a conclusão que a performance da verossimilhança de Xu et al. (2018) tem melhor desempenho mas possui uma maior variância.

A Figura 4.16 apresenta agora a performance das estimativas do TSAQV agora via abordagem piecewise. Comparando com a performance da verossimilhança parcial, só existe uma vantagem nas réplicas de tamanho $n=1000$. A proporção média de censura $\bar{\pi}_{C}=25,23 \%$ para as réplicas de tamanho $n=300$ e $\bar{\pi}_{C}=25,89 \%$ para réplicas de tamanho $n=1000$. Nas estimativas via piecewise foi escolhido $\hat{m}=10$ usando dessa vez o critério de Akaike. Sobre os coeficientes de suavização da penalidade, ambos foram fixados iguais a zero. Portanto, não houve penalidade.

A Figura 4.20 apresenta dois gráficos com as curvas estimadas das basais acumuladas. 


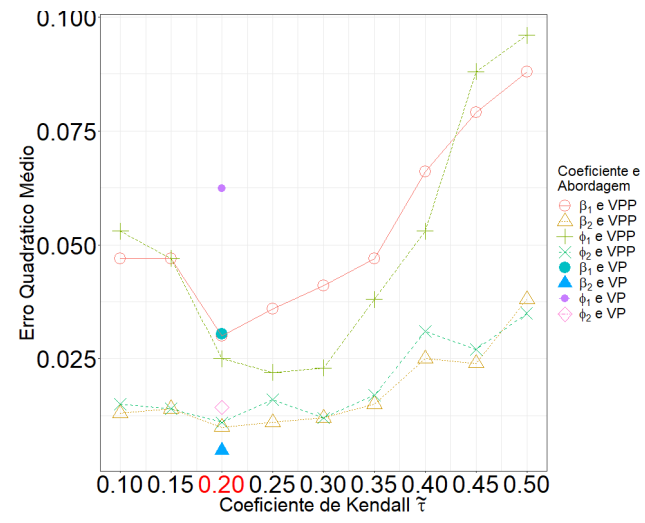

(a) $n=300$

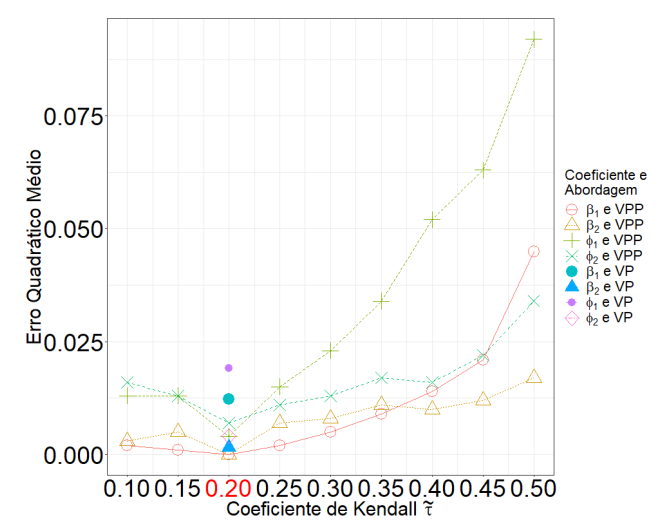

(b) $n=1000$

Figura 4.18: EQM das estimativas para TSAQV cópula Frank e via piecewise

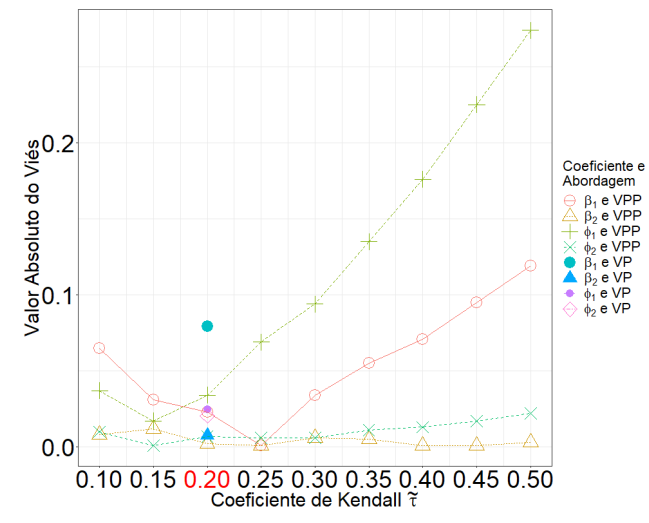

(a) $n=300$

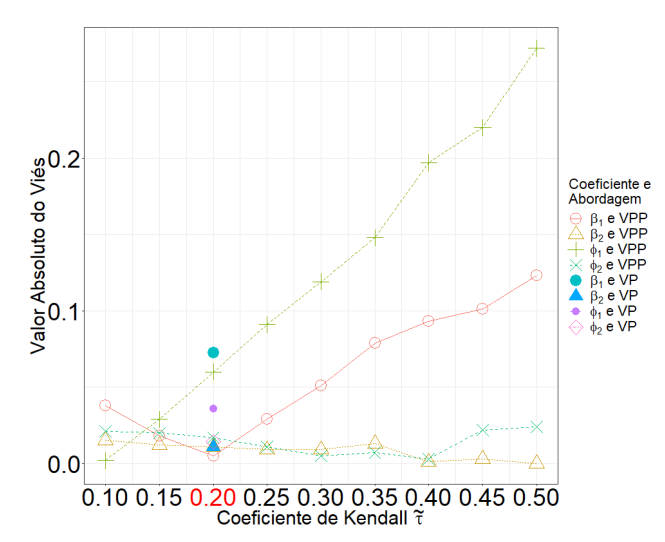

(b) $n=1000$

Figura 4.19: Valor Absoluto do viés das estimativas para TSAQV cópula Frank e via piecewiise

Nota-se que a melhor performance é pela abordagem $M$-splines. A performance via piecewise talvez tenha deixado a desejar devido a escolha de somente 10 intervalos e também pela anulação da penalidade. A curva estimada com a premissa de independência parece sempre subestimar a verdadeira basal.

A conclusão que pode-se chegar é que, para esse cenário estudado, a abordagem $M$-splines parece reproduzir melhores resultados se comparada com os resultados no ajuste de um modelo sob suposição de independência desde que o verdadeiro valor de $\hat{\tau}$ esteja próximo do verdadeiro nível de dependência $\tau$. Essa é uma grande limitação, pois é impossível estimar $\tau$. Um estudo que poderá ser feito no futuro é avaliar, novamente via simulação, caso o estudo de sensibilidade sugeridos pelos autores de fato aponte para valores de $\tau$ próximos aos verdadeiros.

Essa última seção foi escrita para ser uma motivação ao próximo capítulo que tratará de uma análise com dados reais em que os tempos de sobrevivência foram ajustados por escores de QV. Na parte C do Apêndice, estão disponíveis os resultados obtidos pelos processos de estimação utilizando tanto a cópula de Frank quanto a cópula de Clayton. 


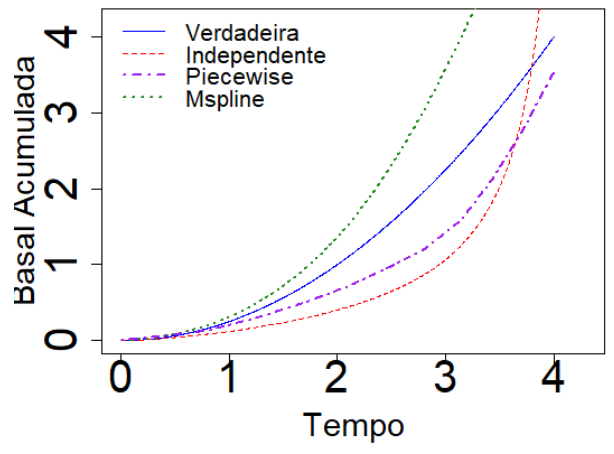

(a) $n=300$

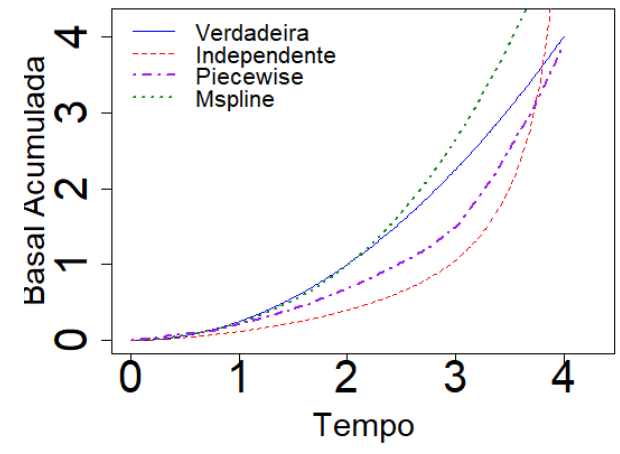

(b) $n=1000$

Figura 4.20: Curvas estimadas das basais acumuladas para TSAQV cópula Frank 


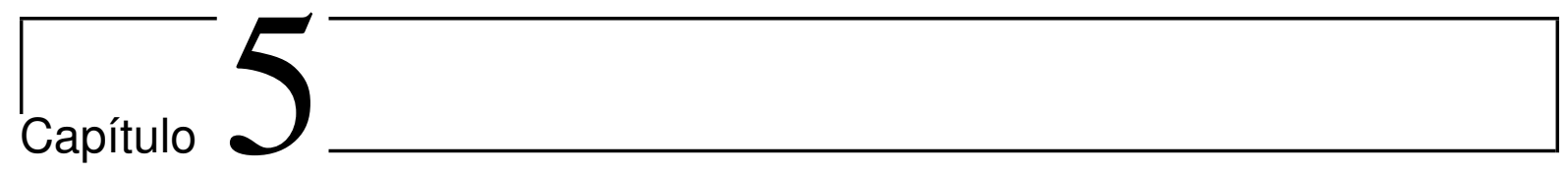

\section{Aplicação: Análise do Tempo de Sobrevivência Ajustado pela Qualidade de Vida em Pacientes com Câncer}

Nesse último capítulo, o interesse é estudar o banco de dados de pacientes com câncer em estágio crítico assistidos pelas unidades de tratamento intensivo (UTI) do Instituto do Câncer do Estado de São Paulo (ICESP) e do Hospital do Câncer de Barretos.

\subsection{Pacientes com Câncer em Estágio Crítico}

Para iniciar uma análise, é preciso uma apresentação mais detalhada dos dados. Para tanto, foram utilizados como suporte os trabalhos de Cesar (2013) e Normilio-Silva et al. (2016) que avaliaram o conjunto de dados também no contexto do TSAQV. Os dados foram coletados por Normilio-Silva et al. (2016) para um estudo de coorte prospectivo em Unidade Intensiva de Tratamento (UTI) nos hospitais públicos Instituto do Câncer Dr. Otávio Frias de Oliveira (ICESP) e pela Fundação Pio XII do Hospital do Câncer de Barretos (São Paulo).

Eles selecionaram apenas pacientes maiores de 18 anos que foram diagnosticados com algum tipo de câncer maligno e que também foram levados para UTI em até 24 horas. Inicialmente, foram pré-selecionados 808 candidatos a participarem do estudo mas somente 792 (98\%) ficaram aptos a prosseguirem por cumprirem pré-requisitos. Esses pacientes foram acompanhados durante 24 meses. Doze pacientes desistiram do acompanhamento após admissão, três pacientes não consentiram em participar do estudo e somente um paciente foi excluído por ter entrado na UTI após 24 horas. A proporção de censura total é 33,96\%. Sob gênero, o conjunto de dados é composto por $42,2 \%$ de mulheres. Para mulheres, foram $34,43 \%$ censuradas versus $33,62 \%$ de censura entre os homens.

A Tabela 5.1 apresenta as descrições das variáveis preditoras escolhidas para uma posterior regressão dos tempos de sobrevivência ajustados pela qualidade de vida neste capítulo. A única preditora quantitativa é a variável Idade. Com exceção das variáveis Idade e Sexo, as demais 
Tabela 5.1: Descrição das variáveis preditoras selecionadas

\begin{tabular}{|c|c|}
\hline Variável & Descrição \\
\hline Sexo & Gênero do paciente. Nominal: Feminino (Femi .) e Masculino (Masc.). \\
\hline Adm & $\begin{array}{l}\text { Forma de admissão do paciente. Nominal: } \\
\text { Eletivo (Elet.), Emergência (Emer.) e Médico (Medi .). }\end{array}$ \\
\hline Ext & $\begin{array}{l}\text { Extensão do câncer para o paciente admitido. Nominal: Metástase (Meta.), } \\
\text { Limitado (Limit.) e Localmente Avançado (Loc. A.). }\end{array}$ \\
\hline SAPS3 & $\begin{array}{l}\text { Escore Fisiológico Agudo Simplificado. Classe: } \geq 18 \text { até }<38\left(\text { SAPS }_{1}\right) \text {, } \\
\geq 38 \text { até }<54\left(\text { SAPS }_{2}\right) \text { e } \geq 54 \text { até }<93\left(\text { SAPS }_{3}\right) \text {. }\end{array}$ \\
\hline ECOG & $\begin{array}{l}\text { Status de desempenho para descrever a capacidade do paciente, por exemplo, } \\
\text { para exercer atividade física ou cuidar de si mesmo ou atividade diária. } \\
\text { Nominal: Completamente Ativo (Ativ.), Restrito para uma Atividade } \\
\text { Rigorosa (Rest.), Ambulatório com Capacidade de se cuidar mas Restrito } \\
\text { a Atividade Rigorosa (Inca.), Capaz de se Cuidar mas Confinado a uma } \\
\text { Cama/Cadeira em mais de 50\% do acompanhamento (Conf.) } \\
\text { e Totalmente Inativo (Inat.). }\end{array}$ \\
\hline Quimio & $\begin{array}{l}\text { Procedimento prévio. Nominal: Curativo/Adjuvante (C.Ad.Q.), } \\
\text { Sem Terapia (Quim.) e Paliativo (Pali.Q.) }\end{array}$ \\
\hline Radio & $\begin{array}{l}\text { Procedimento prévio. Nominal: Curativo/Adjuvante (C.Ad.R.), } \\
\text { Sem Terapia (Radi.) e Paliativo (Pali.R.) }\end{array}$ \\
\hline Idade & Idade dos pacientes (variável quantitativa). \\
\hline
\end{tabular}

foram escolhidas porque Normilio-Silva et al. (2016) encontraram um particular grau de forte associação com uma significante diferença no TSAQV entre as categorias destas variáveis. $\mathrm{Na}$ Tabela 5.2 são apresentadas descrições da frequência relativa para demais variáveis preditoras selecionadas.

Tabela 5.2: Frequência relativa das demais variáveis preditoras

\begin{aligned} & \hline \hline Variável \multicolumn{1}{c}{ Descrição } \\ & \hline Adm: $50,76 \%$ Eletivo, 4,92\% Emergência e 44,32\% Médico. \\ & Ext: $31,56 \%$ Metástase, 32,52\% Limitado e 35,86\% Localmente Avançado. \\ & SAPS3: $30,81 \%$ em $[18,38), 35,23 \%$ em $[38,54)$ e 33,96\% em $[54,93) \\ &$. ECOG: $40,4 \%$ Ativo, 27,02\% Restrito, $14,65 \%$ Incapaz, 12,37\% Confinado e \\ & $5,56 \%$ Inativo. \\ & Quimio: $27,27 \%$ Curativo/Adjuvante e $61,87 \%$ Sem Terapia e 10,86\% Paliativo. \\ & Radio: $18,43 \%$ Curativo/Adjuvante e $78,41 \%$ Sem Terapia e 3,16\% Paliativo. \\ & \hline \hline\end{aligned}

Para coletar os escores de qualidade de vida de cada paciente, Normilio-Silva et al. (2016) aplicaram o questionário EQ-5D-3L que está disponível no apêndice em NormilioSilva (2015). O questionário EQ-5D-3L é composto por questões relacionadas a qualidade de vida em 5 domínios: mobilidade, autocuidados, atividades cotidianas, dor/desconforto, ansiedade/depressão. As respostas são combinadas em uma equação que fornece um escore. Para o questionário EQ-5D-3L, os escores variam de -0,56 a 1 . Um escore igual a 1 indica a 
melhor qualidade de vida possível. Um escore igual a zero indica óbito e um escore negativo indica situações relatadas como piores do que a óbito. Como as metodologias de análise de sobrevivência e qualidade de vida assumem que os escores devem estar no intervalo [0,1], foi feita uma adaptação e os escores negativos foram considerados iguais a zero. Outra adaptação foi somar um valor ínfimo $\varepsilon \sim$ Uniforme $\left(10^{-6}, 2 \cdot 10^{-6}\right)$ para os tempos de sobrevivência para não permitir empates entre os tempos de falhas e tempos de censura para então calcular o estimador de Zhao e Tsiatis para função de sobrevivência. Essa adaptação também ajudou, além da correção da violação de $S_{T}(0)=1$, nas estimativas usando a função de log-verossimilhança de $\mathrm{Xu}$ et al. (2018) que também teve dificuldade em lidar com os empates de pacientes com tempos de sobrevida iguais a zero. O TSAQV para cada paciente foi calculado pelos escores

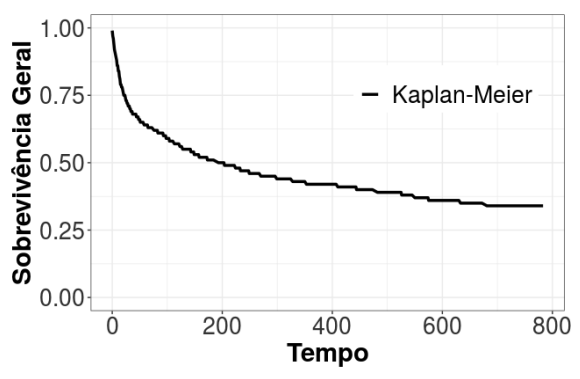

(a) Kaplan-Meier

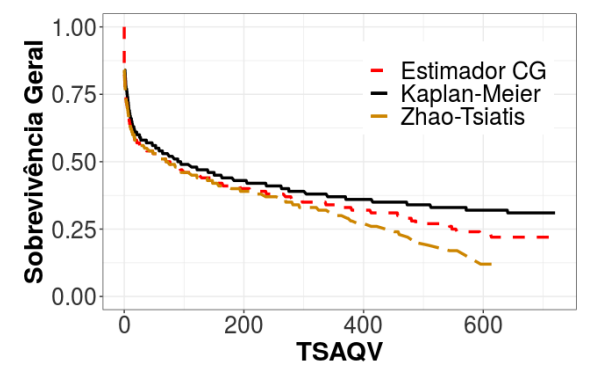

(b) Kaplan-Meier Versus Outros Estimadores

Figura 5.1: Tempo de Sobrevivência e Tempo Ajustado pelos Escores QV

obtidos no momento da entrada na UTI, após 15 dias, três meses, seis meses, doze meses e 18 meses. Em Cesar (2013), os escores QV no décimo oitavo mês não foram considerados porque os dados ainda estavam sendo coletados, em que no décimo oitavo mês seria o equivalente a 540 dias considerando que cada mês corresponde a 30 dias.

Tabela 5.3: Número de pacientes em risco no tempo $t$ (em dias)

\begin{tabular}{l|cccccccc}
\hline \hline Tempo $t$ & 0 & 100 & 200 & 300 & 400 & 500 & 600 & 700 \\
\hline TS & 792 & 471 & 397 & 352 & 329 & 308 & 284 & 269 \\
TSAQV & 792 & 365 & 309 & 259 & 210 & 153 & 92 & 17 \\
\hline \hline
\end{tabular}

No item (a) da Figura 5.1a apresenta a curva da sobrevivência dos tempos sem ajuste via Kaplan-Meier. Agora, levando em consideração os escores de qualidade de vida, usar o estimador de Kaplan-Meier pode ser superestimar a curva de sobrevivência e isso foi evidenciado pelo item (b) da Figura $5.1 \mathrm{~b}$ quando a curva é comparada com a curva tracejada em vermelho que é a curva obtida pelo estimador Gráfico-Cópula em Rivest e Wells (2001) assumindo a cópula Frank como verdadeira a um nível de dependência $\tau=0,80$. Como complemento à Figura 5.1, a Tabela 5.3 apresenta os números de pacientes em risco no tempo $t$ em dias. A linha TS é para o item (a) e a outra linha é para o item (b). Ainda sobre o Gráfico-Copula (CG), admitir que a cópula verdadeira seja Frank mais o nível de dependência para um alto grau é até esse momento uma forte pressuposição. Todavia, por ora, é preservada essa possibilidade como exercício para compreender que o estimador de Kaplan-Meier pode levar a conclusões 
muito equivocadas, sendo o destaque com uma maior diferença observada com base no resultado obtido em Normilio-Silva et al. (2016) usando o estimador de Zhao e Tsiatis. O estimador Gráfico-Cópula em Rivest e Wells (2001) ou estimador CG é definido como

$$
\hat{S}_{T}(t)=\phi_{\alpha}^{-1}\left[\sum_{t_{i} \leq t, \delta_{i}=1} \phi_{\alpha}\left(\frac{n_{i}-1}{n}\right)-\phi_{\alpha}\left(\frac{n_{i}}{n}\right)\right],
$$

em que $\phi_{\alpha}(t)$ é uma função geradora de uma cópula com parâmetro $\alpha, 0 \leq t \leq \max _{i}\left(t_{i}\right)$, $n_{i}=\sum_{u=1}^{n} \mathbf{I}\left(t_{u} \geq t_{i}\right)$ é o número de indivíduos em risco no tempo $t_{i}, \hat{S}(t)=1$ se não ocorreu nenhuma morte no tempo $t$ e $\hat{S}(t)$ é indefinido para $t>\max _{i}\left(t_{i}\right)$. De certa forma, o estimador $C G$ é limitado a forte suposição da cópula verdadeira e também não possui atributo para aplicações em dados que contenham variáveis preditoras. Por outro lado, produz uma curva da função de sobrevivência geral mais suave que a curva pelo estimador de Zhao e Tsiatis. Foi decidido não apresentar os gráficos das curvas estimadas para cada variável preditora devido a já estarem no trabalho de Normilio-Silva et al. (2016).

A seguir, será iniciado o ajuste dos dados ICESP com a função de log-verossimilhança em Xu et al. (2018).

\subsubsection{Ajuste dos Dados ICESP}

Foi decidido usar somente o modelo em Xu et al. (2018) para ajustar os dados efetivamente observáveis do ICESP usando as aproximações lineares às funções basais. A Seção 4.2 faz crer que o ajuste com a log-verossimilhança paramétrica não teria uma boa performance tendo em vista que a combinação dos escores afeta diretamente a verdadeira distribuição dos tempos de falha. Já o modelo em Huang e Zhang (2008) não foi escolhido por conta da morosidade que o algoritmo necessita para obter um $\hat{\tau}$ a partir de um índice $C V(\tau)$ que será explicado no decorrer.

Antes de apresentar os resultados, é preciso voltar a discussão acerca do parâmetro $\alpha$ da cópula arquimediana porque o parâmetro $\alpha$ não é identificável na função de log-verossimilhança. Uma saída é assumir alguns valores para $\alpha$ e estudar o perfil das estimativas para cada coeficiente de regressão como fez Xu et al. (2018). Entretanto, no trabalho de Emura e Chen (2018) foi apresentada mais uma abordagem para mensurar $\alpha$. Emura e Chen (2018) afirmam que a grande vantagem dessa abordagem é que sua interpretação não depende de uma específica função de log-verossimilhança. Essa alternativa é uma metodologia adaptada do Índice-c de Harrell et al. (1982). Aqui é nomeado por índice $C V(\tau)$ e basicamente a técnica consiste em calcular uma validação cruzada em $K$-folds. O primeiro passo é gerar uma amostra para teste tomando $m$ indivíduos da amostra de tamanho $n$. Aqui foi usado $m=\frac{n}{2}$ devido a $n$ ser razoavelmente grande no conjunto de dados ICESP, mas pode ser usado um valor maior como $70 \%$. Como essa amostra de tamanho $m$ vem de uma amostra de tempos de sobrevivência, é necessário certificar que a randomização seja eficiente. Para tanto, foram selecionados pacientes aleatoriamente de tal sorte que a proporção de censura na amostra de tamanho $m$ esteja muito próxima da proporção de censura na amostra de tamanho $n$. Em seguida, fatia-se aquela 
amostra de tamanho $m$ em $K$ grupos tal que a função $\kappa:\{1, \ldots, n\} \longrightarrow\{1, \ldots, K\}$ especifica qual grupo cada paciente foi alocado. Então é definida

$$
P I_{i}(\alpha)=\hat{\boldsymbol{\beta}}_{-\kappa(i)}^{\top} \mathbf{Z}_{i}=\hat{\beta}_{1,-\kappa(i)} Z_{1 i}+\cdots+\hat{\beta}_{p,-\kappa(i)} Z_{p i}
$$

em que $\hat{\boldsymbol{\beta}}_{-\kappa(i)}^{\top}$ será obtido por 3.13 extraindo da amostra de teste o grupo $\kappa(i)$ que o $i$-ésimo paciente foi alocado. A função $P I_{i}(\alpha)$ deve ser interpretada como a função preditora do resultado do par $\left(t_{i}, \delta_{j}\right)$ para o $i$-ésimo paciente. Então Emura e Chen (2018) definem o coeficiente

$$
C V(\alpha)=\frac{\sum_{i<j} \mathbf{I}\left(t_{i}<t_{j}\right) \mathbf{I}\left(P I_{i}(\alpha)>P I_{j}(\alpha)\right) \delta_{i}+\left(t_{j}<t_{i}\right) \mathbf{I}\left(P I_{j}(\alpha)>P I_{i}(\alpha)\right) \delta_{j}}{\sum_{i<j} \mathbf{I}\left(t_{i}<t_{j}\right) \delta_{i}+\mathbf{I}\left(t_{j}<t_{i}\right) \delta_{j}}
$$

e então $\hat{\alpha}$ é o valor $\alpha$ que maximiza $C V(\alpha)$. Foi seguido a recomendação de usar $K=5$ e $G=20$ para obter a estimativa de $\alpha$.

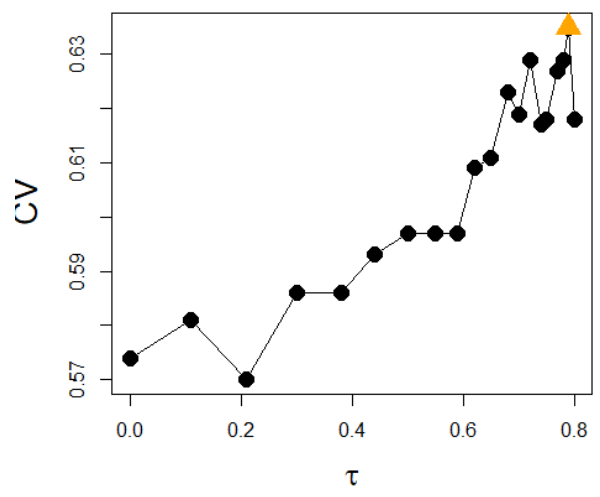

Figura 5.2: Validação cruzada para obter o nível de dependência $\tau$

A Figura 5.2 apresenta a validação cruzada com 5 - folds e $G=20$. Foi construído um algoritmo particular adaptado para a log-verossimilhança de $\mathrm{Xu}$ et al. (2018) descrita no Capítulo 3. No decorrer do texto, fica subentendido que todos os resultados foram obtidos admitindo a cópula de Frank. O valor que maximizou $C V$ foi $\alpha \approx 17,24$ o que significa que o coeficiente de Kendall $\tau=0,79$ indicando uma forte dependência entre o tempo falha ajustado com o tempo de censura ajustada.

Foram escolhidas as seguintes categorias como referência sob critério do menor risco de falhar: Masc., Elet., Ativ., Limit., C.Ad.Q., Radi. e SAPS3 ${ }_{1}$. A Tabela 5.4 apresenta o resumo da performance utilizando modelo com censura independente e dependente ( $M$-splines e piecewise). Na Tabela 5.4 , encontram-se as estimativas $\hat{\beta}$, o erro padrão, a estatística $Z$ de Wald e seu valor $p$ para testar a hipótese nula $H_{0}: \beta_{j}=0, j=1, \ldots, 16$. Pelo modelo com censura dependente nas colunas $M$-splines e piecewise, com um nível de significância 0,05, o teste indica que a variável Idade e as categorias Sexo:Femi., Ext:Meta. e Radio:Pali.R. não são 
Tabela 5.4: Estimativas dos coeficientes para os tempos de falha ajustados

\begin{tabular}{|c|c|c|c|c|c|c|c|c|c|c|c|c|}
\hline \multirow[b]{2}{*}{ Parâmetro } & \multicolumn{4}{|c|}{ Independente } & \multicolumn{4}{|c|}{$M-$ splines } & \multicolumn{4}{|c|}{ Piecewise } \\
\hline & $\hat{\beta}$ & $\hat{\sigma}$ & Z & Valor-p & $\hat{\beta}$ & $\hat{\sigma}$ & Z & Valor-p & $\hat{\beta}$ & $\hat{\sigma}$ & Z & Valor-p \\
\hline Femi. & 0,092 & 0,090 & 1,022 & 0,307 & 0,086 & 0,067 & 1,284 & 0,199 & 0,045 & 0,067 & 0,672 & 0,502 \\
\hline Emer. & 0,648 & 0,204 & 3,176 & 0,001 & 0,489 & 0,183 & 2,672 & 0,008 & 0,578 & 0,181 & 3,193 & 0,001 \\
\hline Med. & 0,462 & 0,133 & 3,474 & 0,001 & 0,284 & 0,112 & 2,536 & 0,011 & 0,326 & 0,110 & 2,964 & 0,003 \\
\hline Rest. & 0,305 & 0,117 & 2,607 & 0,009 & 0,223 & 0,093 & 2,398 & 0,016 & 0,353 & 0,092 & 3,837 & $<0,001$ \\
\hline Inca. & 0,623 & 0,132 & 4,720 & $<0,001$ & 0,559 & 0,113 & 4,947 & $<0,001$ & 0,633 & 0,112 & 5,652 & $<0,001$ \\
\hline Conf. & 0,904 & 0,137 & 6,599 & $<0,001$ & 0,910 & 0,127 & 7,165 & $<0,001$ & 0,968 & 0,122 & 7,934 & $<0,001$ \\
\hline Inat. & 1,352 & 0,186 & 7,269 & $<0,001$ & 1,609 & 0,191 & 8,424 & $<0,001$ & 1,363 & 0,184 & 7,408 & $<0,001$ \\
\hline Loc. A. & 0,299 & 0,116 & 2,578 & 0,010 & 0,277 & 0,088 & 3,148 & 0,002 & 0,381 & 0,089 & 4,281 & $<0,001$ \\
\hline Meta. & 0,297 & 0,134 & 2,216 & 0,027 & 0,157 & 0,110 & 1,427 & 0,154 & 0,245 & 0,150 & 1,633 & 0,102 \\
\hline Quim. & 0,277 & 0,116 & 2,388 & 0,017 & 0,366 & 0,102 & 3,588 & $<0,001$ & 0,405 & 0,101 & 4,010 & $<0,001$ \\
\hline Pali.Q. & 0,522 & 0,153 & 3,412 & 0,001 & 0,904 & 0,143 & 6,322 & $<0,001$ & 0,767 & 0,139 & 5,518 & $<0,001$ \\
\hline C.Ad.R. & 0,274 & 0,115 & 2,383 & 0,017 & 0,264 & 0,102 & 2,588 & 0,010 & 0,391 & 0,098 & 3,990 & $<0,001$ \\
\hline Pali.R. & 0,085 & 0,227 & 0,374 & 0,708 & 0,326 & 0,224 & 1,455 & 0,146 & 0,076 & 0,223 & 0,341 & 0,733 \\
\hline $\mathrm{SAPS}_{2}$ & 0,457 & 0,143 & 3,196 & 0,001 & 0,347 & 0,106 & 3,274 & 0,001 & 0,337 & 0,110 & 3,064 & 0,002 \\
\hline $\mathrm{SAPS}_{3}$ & 0,823 & 0,186 & 4,425 & $<0,001$ & 0,919 & 0,153 & 6,007 & $<0,001$ & 0,668 & 0,155 & 4,310 & $<0,001$ \\
\hline Idade & $-0,001$ & 0,003 & $-0,333$ & 0,739 & $-0,004$ & 0,007 & $-0,571$ & 0,568 & $-0,007$ & 0,006 & $-1,167$ & 0,243 \\
\hline
\end{tabular}

significantes. Entretanto, em contraste, o teste usando a função de log-verossimilhança parcial (independente) diz que a categoria Meta. é significante.

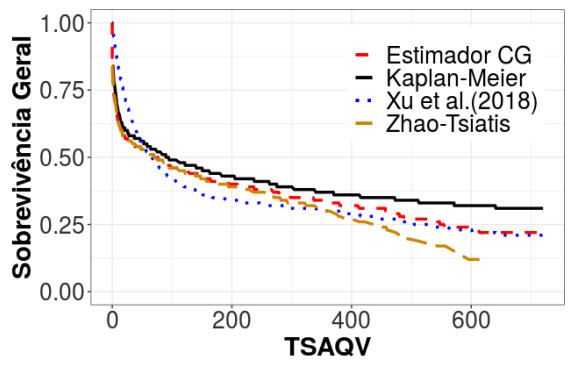

(a) M-splines

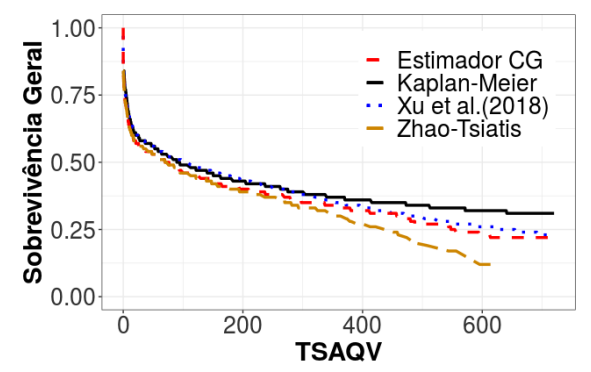

(b) Piecewise

Figura 5.3: Curva de sobrevivência dos tempos ajustados

A Figura 5.3 apresenta as curvas das médias para sobrevivência geral usando dois estimadores não-paramétricos, o estimador CG e a curva usando as estimativas obtidas pela Tabela 5.4 via aproximação por $M$-splines e piecewise. Para o caso do modelo Xu et al. (2018), foi obtida a curva de sobrevivência para cada paciente e a curva apresentada no item (a) da Figura 5.3 é a média de todas as curvas. É notável que todas as curvas indicam que a curva Kaplan-Meier em um determinado momento passa a superestimar a sobrevivência dos pacientes. $\mathrm{O}$ item (b) da Figura 5.3 apresenta as curvas de sobrevivência mas agora pela aproximação via piecewise desenvolvidas no Capítulo 3. Foram selecionados $m=12$ intervalos e a função não foi penalizada, isto é, $h_{1}=h_{2}=0$. A curiosidade é que a curva ficou muito semelhante aquela feita pelo estimador CG. A metodologia para aproximação em si não tem diferença. Tanto as estimativas quanto os resultados dos testes apontam que elas foram bem sucedidas mediante ao algoritmo Multiplicativo Iterativo embora a velocidade das iterações pela abordagem $M$-splines seja menos custosa computacionalmente. 


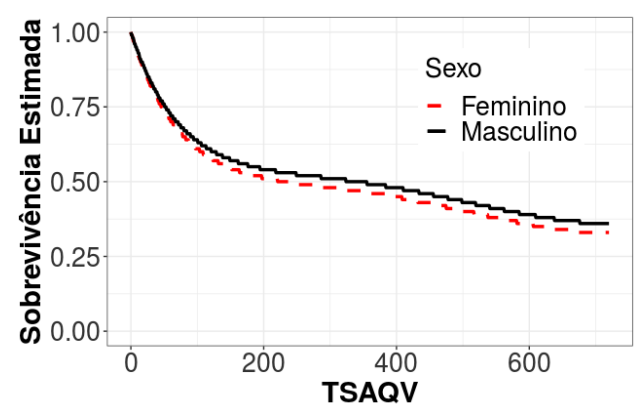

Figura 5.4: Curvas de sobrevivência do TSAQV estimadas por categoria da variável Sexo

Na Figura 5.4 são apresentadas as curvas de sobrevivência do TSAQV estimadas por categoria da variável Sexo. Suponha que tenhamos dois novos pacientes, um para cada sexo, com o mesmo seguinte perfil: sua admissão foi eletiva (Elet.), seu ECOG tem status como ativo (Ativ.), a extensão do câncer é limitada (Limit.), foi induzido a um tratamento curativo/adjuvante prévio ao quimioterápico (C.Ad.Q), não fez radioterapia (Rad), seu o escore SAPS 3 está na classe $[18,38$ ) e tem idade de 61,6 anos. Então, com base nas estimativas obtidas, as curvas da Figura 5.4 predizem a curva da sobrevivência por categoria masculina e feminina dado esse perfil. Nota-se uma sutil diferença de sobrevivência entre masculino e feminino. Por exemplo, no décimo quinto dia, as sobrevivências do masculino e feminino respectivamente são 92\% e 90\%. Já após 18 meses, as sobrevivências estimadas são 46\% e 35\%.

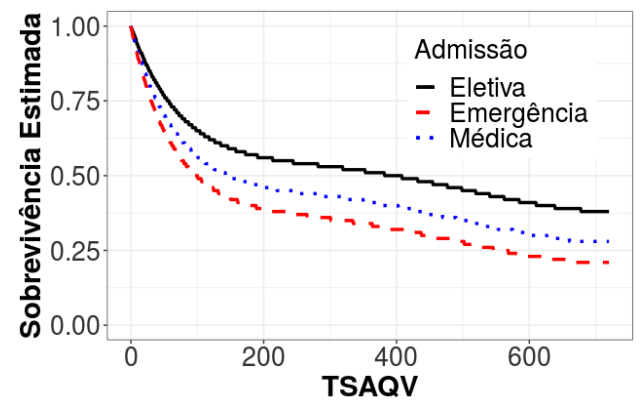

Figura 5.5: Curvas de sobrevivência do TSAQV estimadas por categoria da variável Admissão

Nas últimas seis figuras do capítulo serão destacadas somente as curvas segundos as variáveis que são categorizadas. Somente serão apresentadas as curvas de sobrevivência estimadas via aproximação $M$-splines. Assim como na Figura 5.4 as curvas serão preditoras para a sobrevivência de novos indivíduos sob determinado perfil para cada variável e suas respectivas categorias. A variável Idade no conjunto de dados do ICESP não possui uma prevalência quanto a uma específica faixa etária e, segundo o teste de Wald, não é uma variável significante. Entretanto, assim como a variável Sexo, a variável Idade será utilizada para obter aquelas curvas de sobrevivência a seguir pela média que é aproximadamente 61,6 anos. Portanto, todos os indivíduos serão do sexo masculino, com admissão eletiva, seu ECOG tem status como ativo, com extensão do câncer é limitada, induzido a um tratamento curativo/adjuvante prévio ao quimioterápico, não fez radioterapia, seu o escore SAPS 3 está na classe $[18,38)$ e tem idade 
de 61,6 anos. Esse é o perfil dos indivíduos e agora só cabe particioná-lo convenientemente as categorias de cada variável nominal. Na Figura 5.5 são apresentadas as curvas das categorias da variável Admissão (Adm).

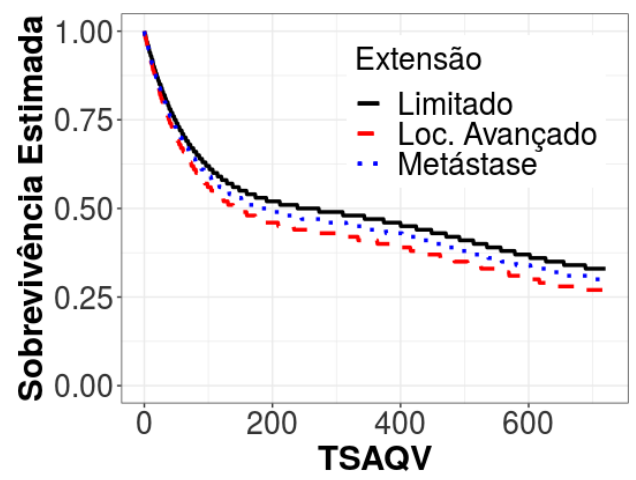

Figura 5.6: Curvas de sobrevivência do TSAQV estimadas por categoria da variável Extensão (do câncer)

Comparando com as curvas obtidas em Normilio-Silva et al. (2016), essas curvas das categorias são mais suaves a longo prazo. Na Figura 5.5 são apresentadas as categorias da variável Extensão de Câncer (Ext) e na Figura 5.7 as curvas estimadas das categorias da variável SAPS3 (SAPS3).

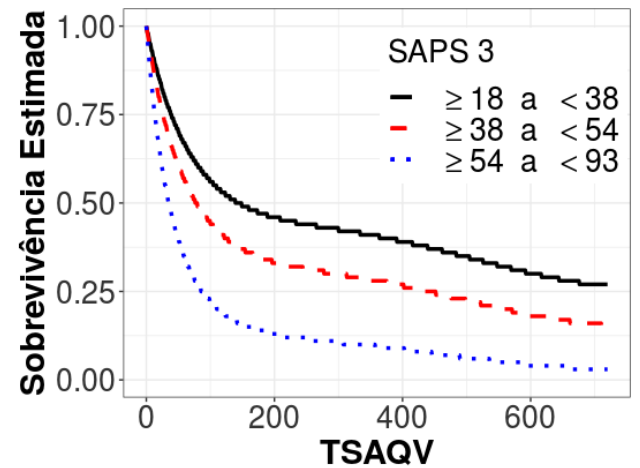

Figura 5.7: Curvas de sobrevivência do TSAQV estimadas por categoria da variável SAPS3

Lembrando que esta análise é gráfica e são apenas levantados questionamentos quanto ao comportamento da curva de sobrevivência. Na Figura 5.7 a variável é SAPS3 e pacientes que obtiveram escores na primeira classe quase que dobram o tempo ajustado de sobrevida se comparado com a última classe.

As Figuras 5.9 5.10 apresentam respectivamente as categorias das preditoras Quimio e Radio. Elas apresentam comportamentos distintos. Enquanto os pacientes que tem maior sobrevida usando tratamento prévio curativo/adjuvante na quimioterapia, para pacientes submetidos a radioterapia a sobrevida dos curativo/adjuvantes são tão ruins quanto os paliativos.

Sobre a aplicação da log-verossimilhança com presença de censura dependente foi satisfatória embora persista algumas dificuldades como o problema de não-identificabilidade do parâmetro $\alpha$ da cópula arquimediana. A alternativa usando o índice $C V(\tau)$ mostrou-se eficiente para descartar a premissa de censura independente. Vale ressaltar que a função de Huang e 


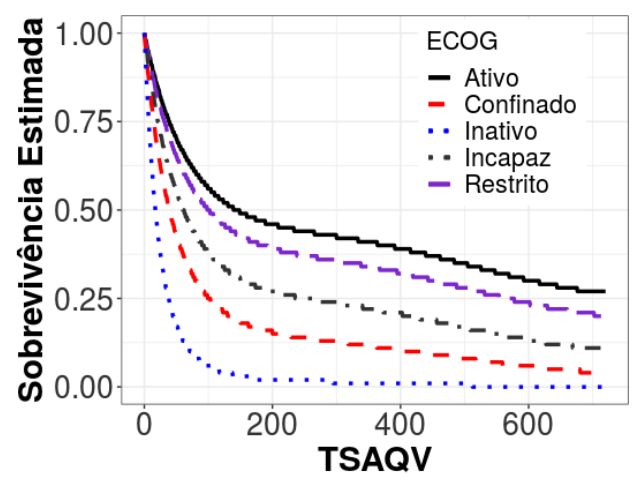

Figura 5.8: Curvas de sobrevivência do TSAQV estimadas por categoria da variável ECOG

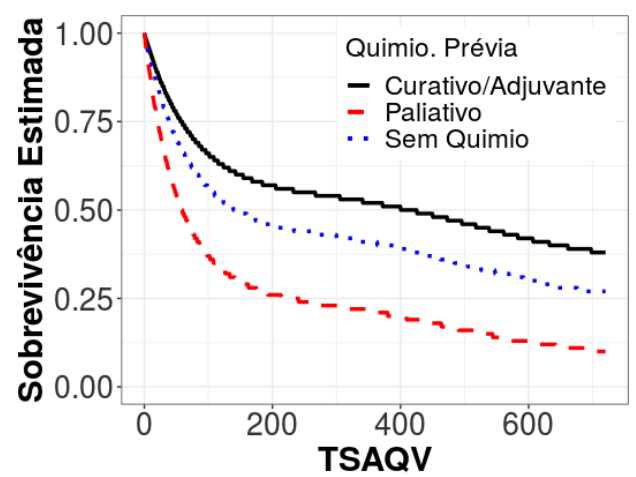

Figura 5.9: Curvas de sobrevivência do TSAQV estimadas por categoria da variável Quimioterapia

Zhang (2008) não foi usada na aplicação, devido somente ao fato do custo computacional que seria exigido na validação cruzada.

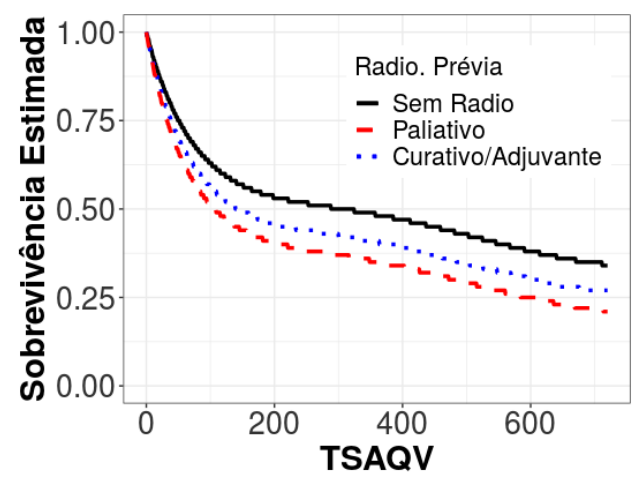

Figura 5.10: Curvas de sobrevivência do TSAQV estimadas por categoria da variável Radioterapia 
Aplicação: Análise do Tempo de Sobrevivência Ajustado pela Qualidade de Vida em 80

Pacientes com Câncer 


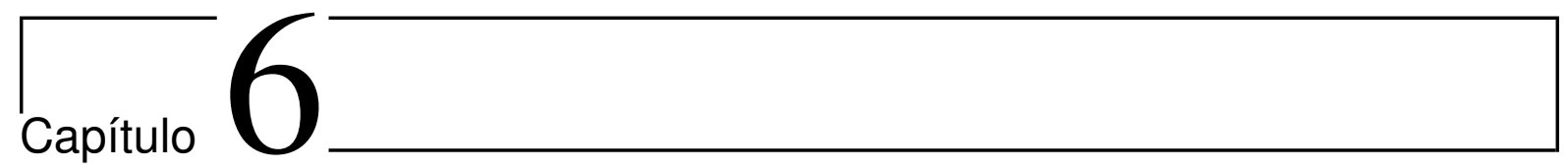

\section{Considerações Finais}

Este trabalho foi desenvolvido tendo como motivação o conjunto de dados do ICESP sendo o principal objetivo a modelagem para dados de sobrevivência com censura dependente assumindo uma função de cópula, obtendo curvas preditoras do tempo de sobrevivência ajustado pela qualidade de vida.

Este texto chegou no seu fim com uma satisfatória aplicação de modelos de sobrevivência assumindo a presença de censura dependente. Entretanto, ainda existem caminhos a serem explorados. Por exemplo, embora admitir uma função cópula no modelo em Xu et al.(2018) retorne uma facilidade na obtenção das estimativas dos coeficientes de regressão, ainda persiste o problema de como comparar as performances entre os diversos membros da classe arquimediana. Outro ponto importante também é estudar melhores abordagens computacionais para reduzir seu custo. Vale ressaltar que o estudo de simulação foi moroso. O cálculo das estimativas usando apenas os algoritmos descritos no Capítulo 3 certamente terá um custo computacional elevado. A principal dificuldade na utilização dos modelos aqui aplicados está no problema de não identificabilidade no parâmetro $\alpha$ da cópula. Um posterior estudo para atualizar possíveis metodologias a respeito dessa dificuldade é necessário. Entretanto, o índice $C V$ apresentou satisfatória performance. Cabe, em um estudo futuro, estudar mais profundamente métodos concorrentes. 
$\Gamma_{\text {Apêndice }} \Lambda$

\section{Principais Resultados para Cópulas Arquimedianas}

Esse primeiro capítulo do Apêndice A é destinado para apresentar os resultados e demonstrações das principais derivadas de uma função de cópula utilizadas neste trabalho.

\section{A.1 Cópula de Clayton}

A seguir, serão apresentados os principais resultados envolvendo derivadas da cópula de Clayton utilizadas neste trabalho. Foi omitido demonstração extensa quando necessário. Em Emura e Chen (2018), a cópula de Clayton é escrita como

$$
K(a, b)=\left(a^{-\alpha}+b^{-\alpha}-1\right)^{-1 / \alpha}, \quad \alpha>0 .
$$

Seguem as derivadas e suas respectivas demonstrações.

$$
\begin{aligned}
K_{1}(a, b) & =\frac{\partial}{\partial a} K(a, b) \\
& =\frac{\partial}{\partial a}\left(a^{-\alpha}+b^{-\alpha}-1\right)^{-1 / \alpha} \\
& =-\frac{1}{\alpha}\left(a^{-\alpha}+b^{-\alpha}-1\right)^{-\frac{1}{\alpha}-1}\left(-\alpha a^{-\alpha-1}\right) \\
& =a^{-(\alpha+1)}\left(a^{-\alpha}+b^{-\alpha}-1\right)^{-\frac{1}{\alpha}-1} \\
& =a^{-(\alpha+1)} K(a, b)[K(a, b)]^{\alpha} \\
& =a^{-(\alpha+1)}[K(a, b)]^{\alpha+1} \\
& =\left[\frac{K(a, b)}{a}\right]^{1+\alpha}
\end{aligned}
$$

e

$$
K_{2}(a, b)=\left.\frac{\partial}{\partial y} K(a, y)\right|_{y=b}=K_{1}(b, a)=\left[\frac{K(a, b)}{b}\right]^{1+\alpha} \text {. }
$$




$$
\begin{aligned}
K_{21}(a, b) & =\frac{\partial}{\partial b} K_{1}(a, b) \\
& =a^{-(1+\alpha)}(1+\alpha)[K(a, b)]^{\alpha} \frac{\partial}{\partial b} K(a, b) \\
& =a^{-(1+\alpha)}(1+\alpha)[K(a, b)]^{\alpha}\left\{b^{-(1+\alpha)}[K(a, b)]^{1+\alpha}\right\} \\
& =(1+\alpha)\left(\frac{K(a, b)}{a b}\right)^{1+\alpha}[K(a, b)]^{\alpha} \\
& =(1+\alpha) \frac{[K(a, b)]^{\alpha}}{b^{1+\alpha}} K_{1}(a, b)
\end{aligned}
$$

e

$$
K_{12}(a, b)=\left.\frac{\partial}{\partial x} K_{2}(x, b)\right|_{x=a}=K_{21}(b, a)=(1+\alpha) \frac{[K(a, b)]^{\alpha}}{a^{1+\alpha}} K_{2}(a, b) .
$$

Consequentemente,

$$
\begin{aligned}
K_{221}= & \frac{\partial}{\partial b} K_{21}(a, b) \\
= & (1+\alpha) \frac{\partial}{\partial b}\left[\left(\frac{K(a, b)}{b}\right)^{\alpha} \times\left(\frac{K_{1}(a, b)}{b}\right)\right] \\
& =(1+\alpha)\left\{\alpha\left(\frac{K(a, b)}{b}\right)^{\alpha-1} \frac{\left[\left(K_{2}(a, b) b-K(a, b)\right)\right]}{b^{2}}\left(\frac{K_{1}(a, b)}{b}\right)-\right. \\
& \left.-\left(\frac{K_{21}(a, b)-K_{1}(a, b)}{b^{2}}\right)\left(\frac{K(a, b)}{b}\right)^{\alpha}\right\}, \\
K_{112} & =\frac{\partial}{\partial a} K_{12}(a, b) \\
= & (1+\alpha) \frac{\partial}{\partial a}\left[\left(\frac{K(a, b)}{a}\right)^{\alpha} \times\left(\frac{K_{2}(a, b)}{a}\right)\right] \\
= & (1+\alpha)\left\{\alpha\left(\frac{K(a, b)}{a}\right)^{\alpha-1} \frac{\left[\left(K_{1}(a, b) a-K(a, b)\right)\right]}{a^{2}}\left(\frac{K_{2}(a, b)}{a}\right)-\right. \\
- & \left.\left(\frac{K_{12}(a, b)-K_{2}(a, b)}{a^{2}}\right)\left(\frac{K(a, b)}{a}\right)^{\alpha}\right\}, \\
& K_{121}=\frac{\partial}{\partial a} K_{21}(a, b) \\
= & \frac{1+\alpha}{b^{1+\alpha}}\left[\alpha\left(\frac{\left[K_{1}(a, b)\right]^{2}}{K(a, b)}\right)+K_{11}(a, b)\right][K(a, b)]^{\alpha}
\end{aligned}
$$


$\mathrm{e}$

$$
\begin{aligned}
K_{212} & =\frac{\partial}{\partial b} K_{12}(a, b) \\
& =\frac{1+\alpha}{a^{1+\alpha}}\left[\alpha\left(\frac{\left[K_{2}(a, b)\right]^{2}}{K(a, b)}\right)+K_{22}(a, b)\right][K(a, b)]^{\alpha}
\end{aligned}
$$

sendo que

$$
\begin{aligned}
K_{11}(a, b) & =\frac{\partial}{\partial a} K_{1}(a, b) \\
& =-(1+\alpha)\left[1-\left(\frac{K(a, b)}{a}\right)^{\alpha}\right] \frac{K_{1}(a, b)}{a} \\
& =\frac{\left(a^{\alpha}-[K(a, b)]^{\alpha}\right)}{a^{1+\alpha}} K_{1}(a, b)
\end{aligned}
$$

e

$$
\begin{aligned}
K_{22}(a, b) & =\frac{\partial}{\partial b} K_{2}(a, b) \\
& =-(1+\alpha)\left[1-\left(\frac{K(a, b)}{b}\right)^{\alpha}\right] \frac{K_{2}(a, b)}{b} \\
& =\frac{\left(b^{\alpha}-[K(a, b)]^{\alpha}\right)}{b^{1+\alpha}} K_{2}(a, b) .
\end{aligned}
$$

Por fim para a cópula de Clayton, tem-se

$$
\begin{aligned}
K_{111}(a, b) & =\frac{\partial}{\partial a} K_{11}(a, b) \\
& =\frac{\partial}{\partial a}-(1+\alpha)\left[\frac{K_{1}(a, b) K(a, b)-a\left[K_{1}(a, b)\right]^{2}}{a K(a, b)}\right] \\
& =-(1+\alpha)\left\{-\alpha\left(\frac{K(a, b)}{a}\right)^{\alpha-1}\left(\frac{a K_{1}(a, b)-K(a, b)}{a^{2}}\right) \frac{K_{1}(a, b)}{a}+\left[1-\left(\frac{K(a, b)}{a}\right)^{\alpha}\right] \times\right. \\
& \left.\times\left(\frac{a K_{11}(a, b)-K_{1}(a, b)}{a^{2}}\right)\right\} \\
& =-\frac{1+\alpha}{a^{\alpha+2}}\left[\left(a^{\alpha}-[K(a, b)]^{\alpha}\right)\left(a K_{11}(a, b)-K_{1}(a, b)\right)-\alpha[K(a, b)]^{\alpha-1} \times\right. \\
& \left.\times\left(a\left[K_{1}(a, b)\right]^{2}-K(a, b) K_{1}(a, b)\right)\right]
\end{aligned}
$$

$\mathrm{e}$

$$
K_{222}(a, b)=\left.\frac{\partial}{\partial y} K_{22}(a, y)\right|_{y=b}=K_{111}(b, a) .
$$

\section{A.2 Cópula de Frank}

A seguir, serão apresentados os principais resultados envolvendo derivadas da cópula de Frank utilizadas neste trabalho. Foi omitido demonstração extensa quando necessário. A cópula de Frank é escrita como 


$$
K(a, b)=\frac{1}{\alpha} \log \left[1+\frac{\left(e^{-\alpha a}-1\right)\left(e^{-\alpha b}-1\right)}{e^{-\alpha}-1}\right] .
$$

Reescrevendo $\xi_{a}=e^{-\alpha a}-1$ e $\xi_{b}=e^{-\alpha b}-1$, tem-se

$$
K(a, b) \propto-\frac{1}{\alpha} \log \left(\xi_{a} \xi_{b}+e^{-\alpha}-1\right)
$$

Implica em

$$
\begin{gathered}
K_{1}(a, b)=\left.\frac{\partial}{\partial x} K(x, b)\right|_{x=a} \\
=-\frac{1}{\alpha} \frac{\xi_{b} \frac{\partial}{\partial a} \xi_{a}}{\xi_{a} \xi_{b}+e^{-\alpha}-1} . \\
K_{2}(a, b)=\left.\frac{\partial}{\partial y} K(a, y)\right|_{y=b} \\
=-\frac{1}{\alpha} \frac{\xi_{a} \frac{\partial}{\partial b} \xi_{b}}{\xi_{a} \xi_{b}+e^{-\alpha}-1} . \\
K_{11}(a, b)=\left.\frac{\partial}{\partial x} K_{1}(x, b)\right|_{x=a} \\
=-\frac{\alpha \xi_{b} e^{-\alpha a}\left(\xi_{a} \xi_{b}+e^{-\alpha}-1\right)-(-\alpha) \xi_{b} e^{-\alpha a} \xi_{b} e^{-\alpha a}}{\left(\xi_{a} \xi_{b}+e^{-\alpha}-1\right)^{2}} \\
=\frac{-\alpha \xi_{a} \xi_{b}^{2}+\alpha\left(1-e^{-\alpha}\right) \xi_{b} e^{-\alpha a}+\alpha \xi_{b}^{2} e^{2-\alpha a}}{\left(\xi_{a} \xi_{b}+e^{-\alpha}-1\right)^{2}} \\
=\frac{\alpha\left(e^{-\alpha a}-\xi_{a}\right) \xi_{b}^{2} e^{-\alpha a}+\alpha\left(1-e^{-\alpha}\right) \xi_{b} e^{-\alpha a}}{\left(\xi_{a} \xi_{b}+e^{-\alpha}-1\right)^{2}} \\
=\frac{\alpha\left[\left(e^{-\alpha a}-\xi_{a}\right) \xi_{b}+\left(1-e^{-\alpha}\right)\right] \xi_{b} e^{-\alpha a}}{\left(\xi_{a} \xi_{b}+e^{-\alpha}-1\right)^{2}} \\
=\frac{\alpha\left(\xi_{b} e^{-\alpha a}+1-e^{-\alpha}-\xi_{a} \xi_{b}\right) \xi_{b} e^{-\alpha a}}{\left(\xi_{a} \xi_{b}+e^{-\alpha}-1\right)^{2}} .
\end{gathered}
$$




$$
\begin{aligned}
K_{22}(a, b) & =\left.\frac{\partial}{\partial x} K_{2}(a, y)\right|_{y=b} \\
& =-\frac{\alpha \xi_{a} e^{-\alpha b}\left(\xi_{a} \xi_{b}+e^{-\alpha}-1\right)-(-\alpha) \xi_{a} e^{-\alpha a} \xi_{a} e^{-\alpha a}}{\left(\xi_{a} \xi_{b}+e^{-\alpha}-1\right)^{2}} \\
& =\frac{-\alpha \xi_{b} \xi_{a}^{2}+\alpha\left(1-e^{-\alpha}\right) \xi_{a} e^{-\alpha b}+\alpha \xi_{a}^{2} e^{2-\alpha b}}{\left(\xi_{a} \xi_{b}+e^{-\alpha}-1\right)^{2}} \\
& =\frac{\alpha\left(e^{-\alpha b}-\xi_{b}\right) \xi_{a}^{2} e^{-\alpha b}+\alpha\left(1-e^{-\alpha}\right) \xi_{a} e^{-\alpha b}}{\left(\xi_{a} \xi_{b}+e^{-\alpha}-1\right)^{2}} \\
& =\frac{\alpha\left[\left(e^{-\alpha b}-\xi_{b}\right) \xi_{a}+\left(1-e^{-\alpha}\right)\right] \xi_{a} e^{-\alpha b}}{\left(\xi_{a} \xi_{b}+e^{-\alpha}-1\right)^{2}} \\
& =\frac{\alpha\left(\xi_{a} e^{-\alpha b}+1-e^{-\alpha}-\xi_{a} \xi_{b}\right) \xi_{a} e^{-\alpha b}}{\left(\xi_{a} \xi_{b}+e^{-\alpha}-1\right)^{2}} .
\end{aligned}
$$

$$
\begin{aligned}
K_{21}(a, b) & =\frac{\partial}{\partial b} K_{1}(a, b) \\
& =\frac{\partial}{\partial b}\left(\frac{\xi_{b} e^{-\alpha a}}{\xi_{a} \xi_{b}+e^{-\alpha}-1}\right) \\
& =\frac{e^{-\alpha a}(-\alpha) e^{-\alpha b}\left(\xi_{a} \xi_{b}+e^{-\alpha}-1\right)-\xi_{a} e^{-\alpha b} \xi_{b} e^{-\alpha a}}{\left(\xi_{a} \xi_{b}+e^{-\alpha}-1\right)^{2}} \\
& =\frac{-\alpha e^{-\alpha(a+b)}\left(\xi_{a} \xi_{b}+e^{-\alpha}-1\right)+\alpha \xi_{a} \xi_{b} e^{-\alpha(a+b)}}{\left(\xi_{a} \xi_{b}+e^{-\alpha}-1\right)^{2}} \\
& =\frac{\alpha\left(\xi_{a} \xi_{b}-\xi_{a} \xi_{b}+1-e^{-\alpha}\right) e^{-\alpha(a+b)}}{\left(\xi_{a} \xi_{b}+e^{-\alpha}-1\right)^{2}} \\
& =\frac{\alpha\left(1-e^{-\alpha}\right) e^{-\alpha(a+b)}}{\left(\xi_{a} \xi_{b}+e^{-\alpha}-1\right)^{2}} .
\end{aligned}
$$

$$
\begin{aligned}
K_{12}(a, b) & =\frac{\partial}{\partial a} K_{2}(a, b) \\
& =\frac{-\alpha e^{-\alpha b} e^{-\alpha b}\left(\xi_{a} \xi_{b}+e^{-\alpha}-1\right)+\alpha e^{-\alpha a} \xi_{b} \xi_{a} e^{-\alpha b}}{\left(\xi_{a} \xi_{b}+e^{-\alpha}-1\right)^{2}} \\
& =\frac{\alpha\left(\xi_{a} \xi_{b}-\xi_{a} \xi_{b}+1-e^{-\alpha}\right) e^{-\alpha(a+b)}}{\left(\xi_{a} \xi_{b}+e^{-\alpha}-1\right)^{2}} \\
& =\frac{\alpha\left(1-e^{-\alpha}\right) e^{-\alpha(a+b)}}{\left(\xi_{a} \xi_{b}+e^{-\alpha}-1\right)^{2}}
\end{aligned}
$$

Com

$$
c_{1}=\alpha\left(1-e^{-\alpha}\right) e^{-\alpha b}
$$

e

$$
\frac{\partial}{\partial a}\left(\xi_{a} \xi_{b}+e^{-\alpha}-1\right)^{2}=-2 \alpha\left(\xi_{a} \xi_{b}+e^{-\alpha}-1\right) \xi_{b} e^{-\alpha a}
$$


tem-se

$$
\begin{aligned}
K_{112}(a, b) & =\frac{\partial}{\partial a} K_{12}(a, b) \\
& =\frac{\partial}{\partial a}\left(\frac{\alpha\left(1-e^{-\alpha} e^{-\alpha(a+b)}\right)}{\left(\xi_{a} \xi_{b}+e^{-\alpha}-1\right)^{2}}\right) \\
& =\alpha\left(1-e^{-\alpha} e^{-\alpha b}\right) \frac{\partial}{\partial a}\left[\frac{e^{-\alpha a}}{\left(\xi_{a} \xi_{b}+e^{-\alpha}-1\right)^{2}}\right] \\
& =c_{1} \frac{\xi_{b} 2 \alpha e^{-2 \alpha a}\left(\xi_{a} \xi_{b}+e^{-\alpha}-1\right)^{2}-\alpha e^{-\alpha a}\left(\xi_{a} \xi_{b}+e^{-\alpha}-1\right)}{\left(\xi_{a} \xi_{b}+e^{-\alpha}-1\right)^{4}}
\end{aligned}
$$

Com $c_{2}=\alpha\left(1-e^{-\alpha}\right) e^{-\alpha a}$,tem-se

$$
\begin{aligned}
K_{12}(a, b)= & K_{21}(a, b) \Longrightarrow K_{121}(a, b)=\frac{\partial}{\partial a} K_{21}(a, b)=K_{112}(a, b)=\frac{\partial}{\partial a} K_{12}(a, b), \\
K_{212}(a, b) & =\frac{\partial}{\partial b} K_{12}(a, b) \\
& =K_{112}(b, a) \\
& =\frac{\xi_{a} 2 \alpha e^{-2 \alpha b} e^{-2 \alpha b}\left(\xi_{a} \xi_{b}+e^{-\alpha}-1\right)^{2}-\alpha e^{-\alpha b}\left(\xi_{a} \xi_{b}+e^{-\alpha}-1\right)}{\left(\xi_{a} \xi_{b}+e^{-\alpha}-1\right)^{4}}
\end{aligned}
$$

$\mathrm{e}$

$$
\begin{aligned}
K_{221}(a, b) & =\left.\frac{\partial}{\partial y} K_{21}(x, y)\right|_{x=b, y=a} \\
& =K_{121}(b, a)
\end{aligned}
$$

Finalmente, para cópula de Frank, tem-se

$$
\begin{aligned}
K_{111}(a, b) & =\frac{\partial}{\partial a} K_{11}(a, b) \\
& =\alpha \xi_{b}\left[\frac{-\alpha e^{-\alpha a}\left(\xi_{b} e^{-\alpha a}+1-e^{-\alpha}-\xi_{a} \xi_{b}\right) \xi_{(a, b)}-e^{-\alpha a}\left(\xi_{b} e^{-\alpha a}+1-e^{-\alpha}-\xi_{a} \xi_{b}\right) \frac{\partial}{\partial a} \xi_{(a, b)}}{\xi_{(a, b)}^{2}}\right] \\
& =\alpha \xi_{b}\left[\frac{\left(-\alpha \xi_{(a, b)}-\frac{\partial}{\partial a} \xi_{(a, b)}\right)\left(\xi_{b} e^{-\alpha a}+1-e^{-a l p h a}-\xi_{a} \xi_{b}\right) e^{-\alpha a}}{\xi_{(a, b)^{2}}}\right] \\
& =-\alpha^{2} \xi_{b}\left[\frac{\left(1-2 \xi_{b} e^{-\alpha a}\right)\left(\xi_{b} e^{-\alpha a}+1-e^{-\alpha}-\xi_{a} \xi_{b}\right) \xi_{(a, b)} e^{-\alpha a}}{\xi_{(a, b)}^{2}}\right]
\end{aligned}
$$

$\mathrm{e}$

$$
K_{222}(a, b)=\frac{\partial}{\partial b} K_{22}(a, b)=K_{111}(b, a),
$$

em que $\xi_{(a, b)}=\left(\xi_{a} \xi_{b}+e^{-\alpha}-1\right)$.

\section{A.3 Cópula de Gumbel-Hougaard}

A seguir, serão apresentados os principais resultados envolvendo derivadas da cópula de Frank utilizadas neste trabalho. Foi omitido demonstração extensa quando necessário. A 
cópula de Gumbel-Hougaard é escrita como

$$
K(a, b)=\exp \left\{-\left[(-\log a)^{\alpha}+(-\log b)^{\alpha}\right]^{\frac{1}{\alpha}}\right\} .
$$

Fazendo $g(a, b)=(-\log a)^{\alpha}+(-\log b)^{\alpha}$, tem-se

$$
\left\{\begin{array}{l}
\frac{\partial}{\partial a} g(a, b)=-\frac{\alpha}{a}(-\log a)^{\alpha-1}, \\
\frac{\partial}{\partial a} g(a, b)=-\frac{\alpha}{b}(-\log b)^{\alpha-1}
\end{array}\right.
$$

então

$$
\left\{\begin{array}{l}
\frac{\partial}{\partial a}[g(a, b)]^{\frac{1}{\alpha}}=-\frac{1}{a}(-\log a)^{\alpha-1}[g(a, b)]^{\frac{1}{\alpha}-1} \\
\frac{\partial}{\partial a}[g(a, b)]^{\frac{1}{\alpha}}=-\frac{1}{b}(-\log b)^{\alpha-1}[g(a, b)]^{\frac{1}{\alpha}-1}
\end{array}\right.
$$

portanto,

$$
\begin{aligned}
& \left\{\begin{array}{l}
K_{1}(a, b)=\frac{1}{a}(-\log a)^{\alpha-1}[g(a, b)]^{\frac{1}{\alpha}-1} K(a, b), \\
K_{2}(a, b)=\frac{1}{b}(-\log b)^{\alpha-1}[g(a, b)]^{\frac{1}{\alpha}-1} K(a, b) .
\end{array}\right. \\
K_{21}(a, b) & =\frac{\partial}{\partial b} K_{1}(a, b) \\
& =\frac{1}{a}(-\log a)^{\alpha-1}\left\{\left(\frac{\alpha-1}{b}\right)(-\log b)^{\alpha-1}[g(a, b)]^{\frac{1}{\alpha}-2} K(a, b)+\right. \\
& \left.+[g(a \cdot b)]^{\frac{1}{\alpha}-1} K_{2}(a, b)\right\} \\
& =\frac{(-\log a)^{\alpha-1}}{a}\left\{\alpha-1+[g(a, b)]^{\frac{1}{\alpha}}\right\} \frac{K_{2}(a, b)}{K(a, b)}
\end{aligned}
$$

e, análogo,

$$
\begin{aligned}
K_{12}(a, b) & =\frac{\partial}{\partial a} K_{2}(a, b) \\
& =\left.\frac{\partial}{\partial y} K_{1}(b, y)\right|_{y=a} \\
& =K_{21}(b, a) \\
& =\frac{(-\log b)^{\alpha-1}}{b}\left\{\alpha-1+[g(a, b)]^{\frac{1}{\alpha}}\right\} \frac{K_{1}(a, b)}{K(a, b)} .
\end{aligned}
$$

Seja

$$
\begin{aligned}
K_{11}(a, b) & =\frac{\partial}{\partial a} K_{1}(a, b) \\
& =\frac{\partial}{\partial a}\left\{\frac{1}{a}(-\log a)^{\alpha-1}[g(a, b)]^{\frac{1}{\alpha}-1} K(a, b)\right\} \\
& =A_{11} K(a, b)+a_{11} K_{1}(a, b),
\end{aligned}
$$


sendo

$$
\begin{aligned}
a_{11} & =a_{11}(a) \\
& =\frac{1}{a}(-\log a)^{\alpha-1}[g(a, b)]^{\frac{1}{\alpha}-1}
\end{aligned}
$$

e

$$
\begin{aligned}
A_{11} & =A_{11}(a) \\
& =\frac{\partial}{\partial a} a_{11} \\
& =\frac{\partial}{\partial a}\left\{\frac{1}{a}(-\log a)^{\alpha-1}[g(a, b)]^{\frac{1}{\alpha}-1}\right\} \\
& =-\frac{1}{a^{2}}(-\log a)^{\alpha-2}\left[\alpha-(1+\log a)+\frac{(1-\alpha)(-\log a)^{\alpha}}{g(a, b)}\right][g(a, b)]^{\frac{1}{\alpha}-1} .
\end{aligned}
$$

Analogamente,

$$
\begin{aligned}
K_{22}(a, b) & =K_{11}(b, a) \\
& =A_{22} K(a, b)+a_{22} K_{2}(a, b),
\end{aligned}
$$

sendo

$$
\begin{aligned}
a_{22} & =a_{11}(b), \\
A_{22} & =A_{11}(b) .
\end{aligned}
$$

$$
\begin{aligned}
K_{111}(a, b) & =\frac{\partial}{\partial a} K_{11}(a, b) \\
& =\frac{\partial}{\partial a}\left\{A_{11} K(a, b)+a_{11} K_{1}(a, b)\right\} \\
& =K(a, b) \frac{\partial}{\partial a} A_{11}+A_{11} K_{1}(a, b)+A_{11} K_{1}(a, b)+a_{11} K_{11}(a, b) \\
& =K(a, b) \frac{\partial}{\partial a}\left(c_{1} d_{1}\right)+A_{11} K_{1}(a, b)+A_{11} K_{1}(a, b)+a_{11} K_{11}(a, b) \\
& =K(a, b)\left(C_{1} d_{1}+c_{1} D_{1}\right)+A_{11} K_{1}(a, b)+A_{11} K_{1}(a, b)+a_{11} K_{11}(a, b) \\
& =2 A_{11} K_{1}(a, b)+a_{11} K_{11}(a, b)+\left(C_{1} d_{1}+c_{1} D_{1}\right) K(a, b),
\end{aligned}
$$

com

$$
\begin{aligned}
& c_{1}=c_{1}(a)=\alpha-(1+\log a)+(1-\alpha) \frac{(-\log a)^{\alpha}}{g(a, b)}, \\
& d_{1}=d_{1}(a)=-\frac{1}{a^{2}}(-\log a)^{\alpha-2}[g(a, b)]^{\frac{1}{\alpha}-1},
\end{aligned}
$$




$$
\begin{aligned}
& \begin{aligned}
C_{1} & =C_{1}(a) \\
& =\frac{\partial}{\partial a} C_{1} \\
& =-\frac{1}{a}+\frac{(1-\alpha) \alpha\left[(-\log a)^{\alpha}-g(a, b)\right](-\log a)^{\alpha-1}}{a^{2}[g(a, b)]^{2}}, \\
D_{1}= & D_{1}(a) \\
= & \frac{\partial}{\partial a} d_{1} \\
= & \frac{(-\log a)^{\alpha-2}}{a^{3}}\left[2-\frac{\alpha-2}{\log a}+\frac{(1-\alpha)(-\log a)^{\alpha-1}}{g(a, b)}\right][g(a, b)]^{\frac{1}{\alpha}-1} .
\end{aligned}
\end{aligned}
$$

De modo análogo,

$$
K_{222}(a, b)=2 A_{22} K_{2}(a, b)+a_{22} K_{22}(a, b)+\left(C_{2} d_{2}+c_{2} D_{2}\right) K(a, b),
$$

sendo

$$
\begin{aligned}
c_{2} & =c_{1}(b), \\
d_{2} & =d_{1}(b), \\
C_{2} & =C_{1}(b), \\
D_{2} & =D_{1}(b) .
\end{aligned}
$$

Agora seja

$$
\begin{aligned}
K_{112}(a, b) & =\frac{\partial}{\partial a} K_{12}(a, b) \\
& =\frac{\partial}{\partial a}\left\{\frac{1}{b}(-\log b)^{\alpha-1}\left\{\alpha-1+[g(a, b)]^{\frac{1}{\alpha}}\right\} \frac{K_{1}(a, b)}{g(a, b)}\right\} \\
& =\frac{1}{b}(-\log b)^{\alpha-1}\left\{\left\{\alpha-1+[g(a, b)]^{\frac{1}{\alpha}}\right\} F_{1}-\frac{1}{a}(-\log a)^{\alpha-1}[g(a, b)]^{\frac{1}{\alpha}-1} f_{1}\right\},
\end{aligned}
$$

sendo

$$
\begin{aligned}
f_{1} & =f_{1}(a) \\
& =\frac{K_{1}(a, b)}{g(a, b)}
\end{aligned}
$$

e

$$
\begin{aligned}
F_{1} & =F_{1}(a) \\
& =\frac{\partial}{\partial a} f_{1} \\
& =\frac{K_{11}(a, b) g(a, b)+\frac{\alpha}{a}(-\log a)^{\alpha-1} K_{1}(a, b)}{[g(a, b)]^{2}} .
\end{aligned}
$$


Analogamente,

$$
\begin{aligned}
K_{221}(a, b) & =\frac{\partial}{\partial b} K_{21}(a, b) \\
& =K_{112}(b, a) \\
& =\frac{1}{a}(-\log a)^{\alpha-1}\left\{\left\{\alpha-1+[g(a, b)]^{\frac{1}{\alpha}}\right\} F_{1}(b)-\frac{1}{b}(-\log b)^{\alpha-1}[g(a, b)]^{\frac{1}{\alpha}-1} f_{1}(b)\right\} .
\end{aligned}
$$

Por fim, sejam

$$
\begin{aligned}
K_{211}(a, b) & =\frac{\partial}{\partial b} K_{11}(a, b) \\
& =\frac{\partial}{\partial b}\left[A_{11} K(a, b)+a_{11} K_{1}(a, b)\right] \\
& =A_{211} K(a, b)+A_{11} K_{2}(a, b)+a_{211} K_{1}(a, b)+a_{11} K_{21},
\end{aligned}
$$

com

$$
\begin{aligned}
& a_{211}=\frac{\partial}{\partial b} a_{11} \\
& =\frac{1}{a}(-\log a)^{\alpha-1}\left(\frac{1-\alpha}{\alpha}\right)[g(a, b)]^{\frac{1}{\alpha-2}}\left(-\frac{\alpha}{a}\right)(-\log a)^{\alpha-1} \\
& =-\frac{(1-\alpha)}{a b}(-\log a)^{\alpha-1}(-\log b)^{\alpha-1}[g(a, b)]^{\frac{1}{\alpha}-2}, \\
& A_{211}=\frac{\partial}{\partial b} A_{11} \\
& =-\frac{(-\log a)^{\alpha-2}}{a}\left\{H_{1}[g(a, b)]^{\frac{1}{\alpha}-1}-h_{1} \frac{(1-\alpha)(-\log a)^{\alpha-1}[g(a, b)]^{\frac{1}{\alpha}-2}}{a}\right\},
\end{aligned}
$$

com

$$
h_{1}=\alpha-(1+\log a)+\frac{(1-\alpha)(-\log a)^{\alpha}}{g(a, b)}
$$

e

$$
\begin{aligned}
H_{1} & =\frac{\partial}{\partial b} h_{1} \\
& =\frac{(1-\alpha) \alpha(-\log a)^{\alpha}(-\log b)^{\alpha-1}}{[b g(a, b)]^{2}} .
\end{aligned}
$$

$$
\begin{aligned}
K_{212}(a, b) & =\frac{\partial}{\partial b} K_{12} \\
& =S_{212} m_{212}+s_{212} M_{212}
\end{aligned}
$$


sendo

$$
\begin{gathered}
b_{212}=\frac{1}{b}(-\log b)^{\alpha-1} K_{1}(a, b), \\
m_{212}=\frac{b_{212}}{g(a, b)}, \\
S_{212}=-\frac{1}{a}(-\log a)^{\alpha-1}[\lg (a, b)]^{\frac{1}{\alpha}-1}, \\
M_{212}=\frac{B_{212} g(a, b)+\frac{\alpha b_{212}}{b}(-\log b)^{\alpha-1}}{[g(a, b)]^{2}},
\end{gathered}
$$

com

$$
B_{212}=-\frac{(-\log b)^{\alpha-1}}{b^{2}}\left(1-\frac{\alpha-1}{\log b}\right) K_{1}(a, b)+\frac{1}{b}(-\log b)^{\alpha-1} K_{21}(a, b) .
$$




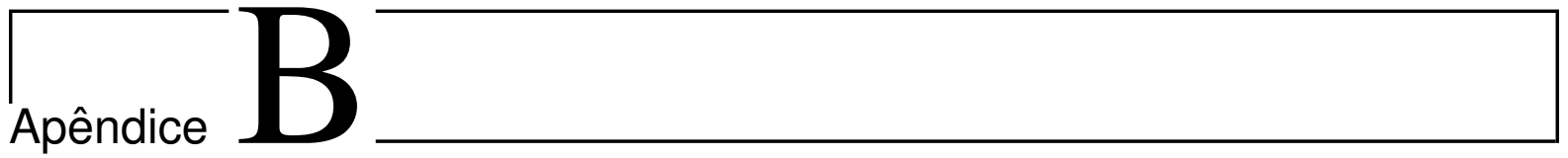

\section{Modelos}

Neste Apêndice B, são apresentados os principais resultados e suas respectivas demonstrações dos modelos de Xu et al. (2018) e Huang e Zhang (2008) discutidos no Capítulo 3. Os vetores gradientes e as matrizes hessianas estão disponíveis a seguir.

\section{B.1 Modelo Xu et al. (2018)}

\section{Vetor Gradiente} ficam

As componentes da função de log-verossimilhança em (3.13) derivadas com relação a $\beta_{j}$,

$$
\begin{aligned}
\frac{\partial}{\partial \beta_{j}} l_{i T} & =Z_{i j}-Z_{i j} \Lambda_{i T}-\frac{K_{11}\left(S_{i T}, S_{i C}\right)}{K_{1}\left(S_{i T}, S_{i C}\right)} \Lambda_{i T} S_{i T} Z_{i j} \\
\frac{\partial}{\partial \beta_{j}} l_{i C} & =-\frac{K_{12}\left(S_{i T}, S_{i C}\right)}{K_{2}\left(S_{i T}, S_{i C}\right)} \Lambda_{i T} S_{i T} Z_{i j} \\
\frac{\partial}{\partial \beta_{j}} l_{i A} & =-\frac{K_{1}\left(S_{i T}, S_{i C}\right)}{K\left(S_{i T}, S_{i C}\right)} \Lambda_{i T} S_{i T} Z_{i j} .
\end{aligned}
$$

Agora seja a $d l_{i}(\boldsymbol{\beta})$ a derivada contribuição $l_{i}$ do $i$-ésimo indivíduo

$$
d l_{i}(\boldsymbol{\beta})=\delta_{i T} \frac{\partial}{\partial \beta_{j}} l_{i T}+\delta_{i C} \frac{\partial}{\partial \beta_{j}} l_{i T}+\left(1-\delta_{i T}-\delta_{i T}\right) \frac{\partial}{\partial \beta_{j}} l_{i A}
$$

então

$$
\begin{aligned}
d l_{i}(\boldsymbol{\beta}) & =\delta_{i T} Z_{i j}-Z_{i j} \delta_{i T} \Lambda_{i T}-\delta_{i T} \frac{K_{11}\left(S_{i T}, S_{i C}\right)}{K_{1}\left(S_{i T}, S_{i C}\right)} \Lambda_{i T} S_{i T} Z_{i j}-\delta_{i C} \frac{K_{12}\left(S_{i T}, S_{i C}\right)}{K_{2}\left(S_{i T}, S_{i C}\right)} \times \\
& \times \Lambda_{i T} S_{i T} Z_{i j}-\left(1-\delta_{i T}-\delta_{i C}\right) \frac{K_{1}\left(S_{i T}, S_{i C}\right)}{K\left(S_{i T}, S_{i C}\right)} \Lambda_{i T} S_{i T} Z_{i j} \\
& =\left[\delta_{i T}-\delta_{i T} \Lambda_{i T}-\left(\delta_{i T} \frac{K_{11}\left(S_{i T}, S_{i C}\right)}{K_{1}\left(S_{i T}, S_{i C}\right)}+\delta_{i C} \frac{K_{12}\left(S_{i T}, S_{i C}\right)}{K_{2}\left(S_{i T}, S_{i C}\right)}+\left(1-\delta_{i T}-\delta_{i C}\right) \frac{K_{1}\left(S_{i T}, S_{i C}\right)}{K\left(S_{i T}, S_{i C}\right)}\right) \Lambda_{i T} S_{i T}\right] Z_{i j} \\
& =\left[\delta_{i T}-\delta_{i T} \Lambda_{i T}-H_{1 i} \Lambda_{i T} S_{i T}\right] Z_{i j},
\end{aligned}
$$


portanto,

$$
\frac{\partial}{\partial \beta_{j}} \Phi(\eta)=\sum_{i=1}^{n} d l_{i}(\boldsymbol{\beta}) .
$$

As componentes da função de log-verossimilhança em (3.13) derivadas com relação a $\phi_{j}$, ficam

$$
\begin{aligned}
\frac{\partial}{\partial \phi_{j}} l_{i C} & =Z_{i j}-Z_{i j} \Lambda_{i C}-\frac{K_{22}\left(S_{i T}, S_{i C}\right)}{K_{2}\left(S_{i T}, S_{i C}\right)} \Lambda_{i C} S_{i C} Z_{i j} \\
\frac{\partial}{\partial \phi_{j}} l_{i T} & =-\frac{K_{21}\left(S_{i T}, S_{i C}\right)}{K_{1}\left(S_{i T}, S_{i C}\right)} \Lambda_{i C} S_{i C} Z_{i j} \\
\frac{\partial}{\partial \phi_{j}} l_{i A} & =-\frac{K_{2}\left(S_{i T}, S_{i C}\right)}{K\left(S_{i T}, S_{i C}\right)} \Lambda_{i C} S_{i C} Z_{i j} .
\end{aligned}
$$

Agora seja a $d g_{i}(\phi)$ a derivada contribuição $l_{i}$ do $i$-ésimo indivíduo

$$
d g_{i}(\phi)=\delta_{i T} \frac{\partial}{\partial \phi_{j}} l_{i T}+\delta_{i C} \frac{\partial}{\partial \phi_{j}} l_{i T}+\left(1-\delta_{i T}-\delta_{i T}\right) \frac{\partial}{\partial \phi_{j}} l_{i A}
$$

então

$$
\begin{aligned}
d g_{i}(\phi) & =\delta_{i C} Z_{i j}-Z_{i j} \delta_{i C} \Lambda_{i C}-\delta_{i C} \frac{K_{22}\left(S_{i T}, S_{i C}\right)}{K_{2}\left(S_{i T}, S_{i C}\right)} \Lambda_{i C} S_{i C} Z_{i j}-\delta_{i T} \frac{K_{21}\left(S_{i T}, S_{i C}\right)}{K_{1}\left(S_{i T}, S_{i C}\right)} \times \\
& \times \Lambda_{i C} S_{i C} Z_{i j}-\left(1-\delta_{i T}-\delta_{i C}\right) \frac{K_{2}\left(S_{i T}, S_{i C}\right)}{K\left(S_{i T}, S_{i C}\right)} \Lambda_{i C} S_{i C} Z_{i j} \\
& =\left[\delta_{i C}-\delta_{i C} \Lambda_{i C}-\left(\delta_{i C} \frac{K_{22}\left(S_{i T}, S_{i C}\right)}{K_{2}\left(S_{i T}, S_{i C}\right)}+\delta_{i T} \frac{K_{21}\left(S_{i T}, S_{i C}\right)}{K_{1}\left(S_{i T}, S_{i C}\right)}+\left(1-\delta_{i T}-\delta_{i C}\right) \frac{K_{2}\left(S_{i T}, S_{i C}\right)}{K\left(S_{i T}, S_{i C}\right)}\right) \Lambda_{i C} S_{i C}\right] Z_{i j} \\
& =\left[\delta_{i C}-\delta_{i C} \Lambda_{i C}-H_{2 i} \Lambda_{i C} S_{i C}\right] Z_{i j},
\end{aligned}
$$

portanto,

$$
\frac{\partial}{\partial \phi_{j}} \Phi(\eta)=\sum_{i=1}^{n} d g_{i}(\phi) .
$$

As componentes da função de log-verossimilhança em (3.13) derivadas com relação a $\theta_{u}$, ficam

$$
\begin{aligned}
\frac{\partial}{\partial \theta_{u}} l_{i T} & =\frac{\psi_{u i}}{\theta_{u}}-\Psi_{i u} e^{Z_{i}^{\top} \beta}-\frac{K_{11}\left(S_{i T}, S_{i C}\right)}{K_{1}\left(S_{i T}, S_{i C}\right)} \Psi_{i u} e^{Z_{i}^{\top} \beta} S_{i T} \\
\frac{\partial}{\partial \theta_{u}} l_{i C} & =-\frac{K_{12}\left(S_{i T}, S_{i C}\right)}{K_{2}\left(S_{i T}, S_{i C}\right)} \Psi_{i u} e^{Z_{i}^{\top} \beta} S_{i T} \\
\frac{\partial}{\partial \theta_{u}} l_{i A} & =-\frac{K_{1}\left(S_{i T}, S_{i C}\right)}{K\left(S_{i T}, S_{i C}\right)} \Psi_{i u} e^{Z_{i}^{\top} \beta_{S}} S_{i T} .
\end{aligned}
$$

Agora são escritas as derivadas utilizando os parâmetros $\theta$ e $\gamma$ da aproximação basal. Seja a $d l_{i}(\theta)$ a derivada contribuição $l_{i}$ do $i$-ésimo indivíduo

$$
d l_{i}(\boldsymbol{\theta})=\delta_{i T} \frac{\partial}{\partial \theta_{u}} l_{i T}+\delta_{i C} \frac{\partial}{\partial \theta_{u}} l_{i C}+\left(1-\delta_{i T}-\delta_{i T}\right) \frac{\partial}{\partial \theta_{u}} l_{i A}
$$


então

$$
\begin{aligned}
d l_{i}(\boldsymbol{\theta}) & =\delta_{i T} \frac{\psi_{i u}}{\theta_{u}}-\delta_{i T} \Psi_{i u} e_{i}^{Z_{i}^{\top} \beta}-\delta_{i T} \frac{K_{11}\left(S_{i T}, S_{i C}\right)}{K_{1}\left(S_{i T}, S_{i C}\right)} \Psi_{i u} \boldsymbol{Z}_{i}^{\top} \beta S_{i T}-\delta_{i C} \frac{K_{12}\left(S_{i T}, S_{i C}\right)}{K_{2}\left(S_{i T}, S_{i C}\right)} \Psi_{i u} e^{Z_{i}^{\top} \beta} S_{i T}- \\
& -\left(1-\delta_{i T}-\delta_{i C}\right) \frac{K_{1}\left(S_{i T}, S_{i C}\right)}{K\left(S_{i T}, S_{i C}\right)} \Psi_{i u} e_{i}^{\top} \beta_{S} S_{i T} \\
& =\delta_{i T} \frac{\psi_{i u}}{\theta_{u}}-\left[\delta_{i T}+\delta_{i T} \frac{K_{11}\left(S_{i T}, S_{i C}\right)}{K_{1}\left(S_{i T}, S_{i C}\right)} S_{i T}+\delta_{i C} \frac{K_{12}\left(S_{i T}, S_{i C}\right)}{K_{2}\left(S_{i T}, S_{i C}\right)} S_{i T}+\left(1-\delta_{i T}-\delta_{i C}\right) \frac{K_{1}\left(S_{i T}, S_{i C}\right)}{K\left(S_{i T}, S_{i C}\right)} S_{i T}\right] \Psi_{i u} \boldsymbol{Z}_{i}^{\top} \boldsymbol{\beta} \\
& =\delta_{i T} \frac{\psi_{i u}}{\theta_{u}}-\left(\delta_{i T}+H_{2 i} S_{i T}\right) \Psi_{i u} e^{Z_{i}^{\top} \boldsymbol{\beta}}
\end{aligned}
$$

portanto,

$$
\frac{\partial}{\partial \theta_{u}} \Phi(\boldsymbol{\eta})=\sum_{i=1}^{n} d l_{i}(\boldsymbol{\theta})+h_{1} \frac{\partial}{\partial \theta_{u}} J(\boldsymbol{\theta}) .
$$
ficam

As componentes da função de log-verossimilhança em (3.13) derivadas com relação a $\gamma_{u}$,

$$
\begin{aligned}
\frac{\partial}{\partial \gamma_{u}} l_{i C} & =\frac{\psi_{u i}}{\gamma_{u}}-\Psi_{i u} e^{Z_{i}^{\top} \phi}-\frac{K_{22}\left(S_{i T}, S_{i C}\right)}{K_{2}\left(S_{i T}, S_{i C}\right)} \Psi_{i u} e^{Z_{i}^{\top} \phi} S_{i C} \\
\frac{\partial}{\partial \gamma_{u}} l_{i T} & =-\frac{K_{21}\left(S_{i T}, S_{i C}\right)}{K_{1}\left(S_{i T}, S_{i C}\right)} \Psi_{i u} e^{Z_{i}^{\top} \phi} S_{i C} \\
\frac{\partial}{\partial \gamma_{u}} l_{i A} & =-\frac{K_{2}\left(S_{i T}, S_{i C}\right)}{K\left(S_{i T}, S_{i C}\right)} \Psi_{i u} e^{Z_{i}^{\top} \phi_{S}} S_{i C} .
\end{aligned}
$$

Agora seja $g l_{i}(\gamma)$ a derivada contribuição $l_{i}$ do $i$-ésimo indivíduo

$$
g l_{i}(\gamma)=\delta_{i T} \frac{\partial}{\partial \gamma_{u}} l_{i T}+\delta_{i C} \frac{\partial}{\partial \gamma_{u}} l_{i C}+\left(1-\delta_{i T}-\delta_{i T}\right) \frac{\partial}{\partial \gamma_{u}} l_{i A}
$$

então

$$
\begin{aligned}
g l_{i}(\gamma) & =\delta_{i C} \frac{\psi_{i u}}{\gamma_{u}}-\delta_{i C} \Psi_{i u} e^{Z_{i}^{\top} \phi}-\delta_{i C} \frac{K_{22}\left(S_{i T}, S_{i C}\right)}{K_{1}\left(S_{i T}, S_{i C}\right)} \Psi_{i u} e_{i}^{Z_{i}^{\top} \phi_{S}} S_{i C}-\delta_{i T} \frac{K_{21}\left(S_{i T}, S_{i C}\right)}{K_{1}\left(S_{i T}, S_{i C}\right)} \Psi_{i u} e^{Z_{i}^{\top} \phi_{S}} S_{i C}- \\
& -\left(1-\delta_{i T}-\delta_{i C}\right) \frac{K_{2}\left(S_{i T}, S_{i C}\right)}{K\left(S_{i T}, S_{i C}\right)} \Psi_{i u} e_{i}^{Z_{i}^{\top} \phi} S_{i C} \\
& =\delta_{i C} \frac{\psi_{i u}}{\gamma_{u}}-\left[\delta_{i C}+\delta_{i C} \frac{K_{22}\left(S_{i T}, S_{i C}\right)}{K_{2}\left(S_{i T}, S_{i C}\right)} S_{i C}+\delta_{i T} \frac{K_{21}\left(S_{i T}, S_{i C}\right)}{K_{1}\left(S_{i T}, S_{i C}\right)} S_{i C}+\left(1-\delta_{i T}-\delta_{i C}\right) \frac{K_{2}\left(S_{i T}, S_{i C}\right)}{K\left(S_{i T}, S_{i C}\right)} S_{i C}\right] \Psi_{i u} e^{Z_{i}^{\top} \gamma} \\
& =\delta_{i C} \frac{\psi_{i u}}{\gamma_{u}}-\left(\delta_{i C}+H_{2 i} S_{i C}\right) \Psi_{i u} e_{i}^{Z_{i}^{\top} \gamma}
\end{aligned}
$$

portanto,

$$
\frac{\partial}{\partial \gamma_{u}} \Phi(\eta)=\sum_{i=1}^{n} d g_{i}(\gamma)+h_{2} \frac{\partial}{\partial \gamma_{u}} J(\gamma)
$$

No caso da aproximação via piecewise, as funções de penalidade

$$
\left\{\begin{array}{l}
J(\boldsymbol{\theta})=\sum_{u=1}^{m}\left(\theta_{u-1}-\theta_{u}\right)^{2} \\
J(\gamma)=\sum_{u=1}^{m}\left(\gamma_{u-1}-\gamma_{u}\right)^{2}
\end{array}\right.
$$


podendo ser reescritas como uma matrizes quadráticas

$$
\left\{\begin{array}{l}
J(\theta)=\theta^{\top} R \boldsymbol{\theta} \\
J(\gamma)=\gamma^{\top} R \gamma
\end{array}\right.
$$

tal que

$$
R=\left[\begin{array}{cccccccc}
1 & -1 & 0 & \cdots & 0 & \cdots & 0 & 0 \\
-1 & 2 & -1 & 0 & \cdots & 0 & \cdots & 0 \\
0 & -1 & 2 & \ddots & 0 & \cdots & 0 & 0 \\
\vdots & 0 & \ddots & \ddots & \ddots & 0 & \cdots & 0 \\
0 & \vdots & 0 & \ddots & \ddots & \ddots & 0 & 0 \\
\vdots & 0 & \vdots & 0 & \ddots & \ddots & \ddots & 0 \\
0 & \vdots & 0 & \vdots & 0 & \ddots & 2 & -1 \\
0 & 0 & 0 & 0 & 0 & 0 & -1 & 1
\end{array}\right]_{m \times m}
$$

Já para a aproximação via $M$-splines, as funções de penalidade também podem ser reescritas como matrizes quadráticas como anteriormente mas com

$$
R=\frac{1}{\Delta^{5}}\left[\begin{array}{ccccc}
192 & -132 & 24 & 12 & 0 \\
-132 & 96 & -24 & -12 & 12 \\
24 & -24 & 24 & -24 & 24 \\
12 & -12 & -24 & 96 & -132 \\
0 & 12 & 24 & -132 & 192
\end{array}\right]_{5 \times 5}
$$

sendo $\Delta=\xi_{2}-\xi_{1}=\xi_{3}-\xi_{2}$.

Segue a demonstração: definem-se as funções de aproximação via $M$-splines $M_{l}$ e $I_{l}$ do Capítulo 3 a seguir

$$
\begin{aligned}
& M_{1}(t)=-\frac{4 \mathbf{I}\left(\xi_{1} \leq t<\xi_{2}\right)}{\Delta}\left[z_{2}(t)\right]^{3}, \\
& M_{2}(t)=\frac{\mathbf{I}\left(\xi_{1} \leq t<\xi_{2}\right)}{2 \Delta}\left\{\left[7 z_{1}(t)\right]^{3}-18\left[z_{1}(t)\right]^{2}+12 z_{1}(t)\right\}-\frac{\mathbf{I}\left(\xi_{2} \leq t<\xi_{3}\right)}{2 \Delta}\left[z_{3}(t)\right]^{3}, \\
& M_{3}(t)=\frac{\mathbf{I}\left(\xi_{1} \leq t<\xi_{2}\right)}{\Delta}\left\{-2\left[z_{1}(t)\right]^{3}+3\left[z_{1}(t)\right]^{2}\right\}+\frac{\mathbf{I}\left(\xi_{2} \leq t<\xi_{3}\right)}{\Delta}\left\{2\left[z_{2}(t)\right]^{3}-3\left[z_{2}(t)\right]^{2}+1\right\}, \\
& M_{4}(t)=\frac{\mathbf{I}\left(\xi_{1} \leq t<\xi_{2}\right)}{2 \Delta}\left\{\left[z_{1}(t)\right]^{3}\right\}+\frac{\mathbf{I}\left(\xi_{2} \leq t<\xi_{3}\right)}{2 \Delta}\left\{-7\left[z_{2}(t)\right]^{3}+3\left[z_{2}(t)\right]^{2}+3 z_{2}(t)+1\right\}, \\
& M_{5}(t)=\frac{4 \mathbf{I}\left(\xi_{2} \leq t<\xi_{3}\right)}{\Delta}\left[z_{2}(t)\right]^{3} .
\end{aligned}
$$

Como as funções de aproximação da basal acumulada são definidas como $I_{l}(t)=\int_{\xi_{1}}^{t} M(w) d w$, consequentemente, tem-se

$$
\begin{aligned}
& I_{1}(t)=1-\left[z_{2}(t)\right]^{4} \mathbf{I}\left(\xi_{1} \leq t<\xi_{2}\right), \\
& I_{2}(t)=\mathbf{I}\left(\xi_{1} \leq t<\xi_{2}\right)\left\{\frac{7}{8}\left[z_{1}(t)\right]^{4}-3\left[z_{1}(t)\right]^{3}+3\left[z_{1}(t)\right]^{2}\right\}+\mathbf{I}\left(\xi_{2} \leq t<\xi_{3}\right)\left\{1-\frac{1}{8}\left[z_{3}(t)\right]^{4}\right\}, \\
& I_{3}(t)=\left\{-\frac{1}{2}\left[z_{1}(t)\right]^{4}+\left[z_{1}(t)\right]^{3}\right\} \mathbf{I}\left(\xi_{1} \leq t<\xi_{2}\right)+\left\{\frac{1}{2}+\frac{1}{2}\left[z_{2}(t)\right]^{4}-\left[z_{2}(t)\right]^{3}+z_{2}(t)\right\} \mathbf{I}\left(\xi_{2} \leq t<\xi_{3}\right), \\
& I_{4}(t)=\frac{1}{8}\left[z_{1}(t)\right]^{4} \mathbf{I}\left(\xi_{1} \leq t<\xi_{2}\right)+\left\{\frac{1}{8}-\frac{7}{8}\left[z_{2}(t)\right]^{4}+\frac{1}{2}\left[z_{2}(t)\right]^{3}+\frac{3}{4}\left[z_{2}(t)\right]^{2}+\frac{1}{2} z_{2}(t)\right\} \mathbf{I}\left(\xi_{2} \leq t \xi_{3}\right), \\
& I_{5}(t)=\left[z_{2}(t)\right]^{4} \mathbf{I}\left(\xi_{2} \leq t<\xi_{3}\right) .
\end{aligned}
$$


Então para obter uma função de penalidade como, por exemplo, a $J(\theta)$ - agora usando aproximação via M-splines — como simples cálculos segue que

$$
\begin{aligned}
J(\boldsymbol{\theta}) & =\sum_{k=1}^{5} \sum_{l=1}^{5} \theta_{k}\left(\int \frac{d^{2}}{d t^{2}} M_{k}(t) \frac{d^{2}}{d t^{2}} M_{l}(t) d t\right) \theta_{l} \\
& =\boldsymbol{\theta}^{\top} \frac{1}{\Delta^{5}}\left[\begin{array}{ccccc}
192 & -132 & 24 & 12 & 0 \\
-132 & 96 & -24 & -12 & 12 \\
24 & -24 & 24 & -24 & 24 \\
12 & -12 & -24 & 96 & -132 \\
0 & 12 & 24 & -132 & 192
\end{array}\right] \boldsymbol{\theta} .
\end{aligned}
$$

Semelhante para $J(\gamma)$ e vamos omitir a demonstração.

\section{Matriz Hessiana $S_{1}$}

A seguir, vamos demonstrar a matriz hessiana $S_{1}$ dos coeficientes de regressão $\beta$ e $\phi$ para os tempos de falha e tempos de censura. Seguem as derivadas de segunda ordem das componentes

$$
\frac{\partial^{2}}{\partial \beta_{r} \partial \beta_{j}}=\sum_{i=1}^{n} \frac{\partial}{\partial \beta_{r}}\left(\delta_{i T}-\delta_{i T} \Lambda_{i T}-H_{1 i} \Lambda_{i T} S_{i T}\right) Z_{i j}
$$

Segue que

$$
\frac{\partial}{\partial \beta_{r}} H_{1 i} \Lambda_{i T} S_{i T}=\Lambda_{i T} S_{i T} \frac{\partial}{\partial \beta_{j}} H_{1 i}+H_{1 i} \frac{\partial}{\partial \beta_{r}}\left(\Lambda_{i T} S_{i T}\right)
$$

e vamos denotar

$$
\begin{aligned}
& K_{i}=K\left(S_{i T}, S_{i C}\right), \\
& K_{1 i}=K_{1}\left(S_{i T}, S_{i C}\right), \\
& K_{2 i}=K_{2}\left(S_{i T}, S_{i C}\right), \\
& K_{11 i}=K_{11}\left(S_{i T}, S_{i C}\right), \\
& K_{12 i}=K_{12}\left(S_{i T}, S_{i C}\right), \\
& \delta_{i A}=1-\delta_{i T}-\delta_{i C}, \\
& \frac{\partial}{\partial \beta_{t}} H_{1 i}=\frac{\partial}{\partial \beta_{r}}\left(\delta_{i T} \frac{K_{11 i}}{K_{1 i}}+\delta_{i C} \frac{K_{12 i}}{K_{2 i}}+\delta_{i A} \frac{K_{1 i}}{K_{i}}\right) \\
&=\delta_{i T} \frac{K_{11 i i} K_{1}-K_{11 i}^{2}}{K_{1 i}^{2}} \frac{\partial}{\partial \beta_{r}} S_{i T}+\delta_{i C} \frac{K_{112 i} K_{2 i}-K_{12 i}^{2}}{K_{2 i}^{2}} \frac{\partial}{\partial \beta_{r}} S_{i T}+\delta_{i A} \frac{K_{11 i} K_{i}-K_{1 i}^{2}}{K_{i}^{2}} \frac{\partial}{\partial \beta_{r}} S_{i T} \\
&=\left(\delta_{i T} \frac{K_{111 i} K_{1}-K_{11 i}^{2}}{K_{1 i}^{2}}+\delta_{i C} \frac{\left.K_{112 i} K_{2 i}-K_{12 i}^{2}+\delta_{i A} \frac{K_{11 i} K_{i}-K_{1 i}^{2}}{K_{i}^{2}}\right) \frac{\partial}{\partial \beta_{r}} S_{i T}}{K_{2 i}^{2}}\right.
\end{aligned}
$$

agora, reescrevendo

$$
\frac{\partial}{\partial S_{i T}} H_{1 i}=\delta_{i T} \frac{K_{111 i} K_{1}-K_{11 i}^{2}}{K_{1 i}^{2}}+\delta_{i C} \frac{K_{112 i} K_{2 i}-K_{12 i}^{2}}{K_{2 i}^{2}}+\delta_{i A} \frac{K_{11 i} K_{i}-K_{1 i}^{2}}{K_{i}^{2}}
$$


fica

$$
\frac{\partial}{\partial \beta_{r}} H_{1 i}=\frac{\partial}{\partial S_{i T}} H_{1 i} \frac{\partial}{\partial \beta_{r}} S_{i T}
$$

Logo,

$$
\begin{aligned}
\frac{\partial^{2}}{\partial \beta_{r} \beta_{j}} \Phi(\eta) & =\sum_{i=1}^{n}\left[-Z_{i r} \delta_{i T} \Lambda_{i T}+\left(Z_{i r} \Lambda_{i T}^{2} S_{i T}^{2} \frac{\partial}{\partial S_{i T}} H_{1 i}+Z_{i r} H_{1 i} \Lambda_{i T} S_{i T}-Z_{i r} \Lambda_{i T}^{2} S_{i T} H_{1 i}\right)\right] Z_{i j} \\
& =-\sum_{i=1}^{n}\left[\delta_{i T}-\left(S_{i T}^{2} \Lambda_{i T} \frac{\partial}{\partial S_{i T}} H_{1 i}+H_{1 i} S_{i T}-\Lambda_{i T} S_{i T} H_{1 i}\right)\right] \Lambda_{i T} Z_{i j} Z_{i r}
\end{aligned}
$$

Similarmente,

$$
\frac{\partial^{2}}{\partial \phi_{r} \partial \phi_{j}} \Phi(\eta)=\sum_{i=1}^{n} \frac{\partial}{\partial \phi_{r}}\left(\delta_{i C}-\delta_{i C} \Lambda_{i C}-H_{2 i} \Lambda_{i C} S_{i C}\right) Z_{i j}
$$

Segue que

$$
\frac{\partial}{\partial \phi_{r}} H_{2 i} \Lambda_{i C} S_{i C}=\Lambda_{i C} S_{i C} \frac{\partial}{\partial \phi_{r}} H_{2 i}+H_{2 i} \frac{\partial}{\partial \phi_{r}}\left(\Lambda_{i C} S_{i C}\right)
$$

e vamos denotar

$$
\begin{aligned}
K_{i} & =K\left(S_{i T}, S_{i C}\right), \\
K_{1 i} & =K_{1}\left(S_{i T}, S_{i C}\right), \\
K_{2 i} & =K_{2}\left(S_{i T}, S_{i C}\right), \\
K_{22 i} & =K_{11}\left(S_{i T}, S_{i C}\right), \\
K_{21 i} & =K_{21}\left(S_{i T}, S_{i C}\right), \\
\delta_{i A} & =1-\delta_{i T}-\delta_{i C},
\end{aligned}
$$

$$
\begin{aligned}
\frac{\partial}{\partial \phi_{r}} H_{2 i} & =\frac{\partial}{\partial \phi_{r}}\left(\delta_{i C} \frac{K_{22 i}}{K_{2 i}}+\delta_{i T} \frac{K_{21 i}}{K_{1 i}}+\delta_{i A} \frac{K_{2 i}}{K_{i}}\right) \\
& =\delta_{i C} \frac{K_{222 i} K_{1}-K_{22 i}^{2}}{K_{2 i}^{2}} \frac{\partial}{\partial \beta_{r}} S_{i C}+\delta_{i T} \frac{K_{221 i} K_{1 i}-K_{21 i}^{2}}{K_{1 i}^{2}} \frac{\partial}{\partial \phi_{r}} S_{i C}+\delta_{i A} \frac{K_{22 i} K_{i}-K_{2 i}^{2}}{K_{i}^{2}} \frac{\partial}{\partial \phi_{r}} S_{i C} \\
& =\left(\delta_{i C} \frac{K_{222 i} K_{1}-K_{22 i}^{2}}{K_{2 i}^{2}}+\delta_{i T} \frac{K_{121 i} K_{1 i}-K_{21 i}^{2}}{K_{1 i}^{2}}+\delta_{i A} \frac{K_{22 i} K_{i}-K_{2 i}^{2}}{K_{i}^{2}}\right) \frac{\partial}{\partial \phi_{r}} S_{i C}
\end{aligned}
$$

agora, reescrevendo

$$
\frac{\partial}{\partial S_{i C}} H_{2 i}=\delta_{i C} \frac{K_{222 i} K_{1}-K_{22 i}^{2}}{K_{2 i}^{2}}+\delta_{i T} \frac{K_{121 i} K_{1 i}-K_{21 i}^{2}}{K_{1 i}^{2}}+\delta_{i A} \frac{K_{22 i} K_{i}-K_{2 i}^{2}}{K_{i}^{2}}
$$

fica

$$
\frac{\partial}{\partial \phi_{r}} H_{2 i}=\frac{\partial}{\partial S_{i C}} H_{2 i} \frac{\partial}{\partial \phi_{r}} S_{i C}
$$


Logo,

$$
\begin{aligned}
\frac{\partial^{2}}{\partial \phi_{r} \phi_{j}} \Phi(\eta) & =\sum_{i=1}^{n}\left[-Z_{i r} \delta_{i C} \Lambda_{i C}+\left(Z_{i r} \Lambda_{i C}^{2} S_{i C}^{2} \frac{\partial}{\partial S_{i C}} H_{2 i}+Z_{i r} H_{2 i} \Lambda_{i C} S_{i C}-Z_{i r} \Lambda_{i C}^{2} S_{i C} H_{2 i}\right)\right] Z_{i j} \\
& =-\sum_{i=1}^{n}\left[\delta_{i C}-\left(S_{i C}^{2} \Lambda_{i C} \frac{\partial}{\partial S_{i C}} H_{2 i}+H_{2 i} S_{i C}-\Lambda_{i C} S_{i C} H_{2 i}\right)\right] \Lambda_{i C} Z_{i j} Z_{i r} .
\end{aligned}
$$

Finalmente,

$$
\frac{\partial^{2}}{\partial \phi_{r} \partial \beta_{j}}=\sum_{i=1}^{n} \frac{\partial}{\partial \phi_{r}}\left(\delta_{i T}-\delta_{i T} \Lambda_{i T}-H_{1 i} \Lambda_{i T} S_{i T}\right) Z_{i j}
$$

Segue que

$$
\frac{\partial}{\partial \phi_{r}} H_{1 i} \Lambda_{i T} S_{i T}=\Lambda_{i T} S_{i T} \frac{\partial}{\partial \phi_{r}} H_{1 i}
$$

e vamos denotar

$$
\begin{aligned}
K_{i} & =K\left(S_{i T}, S_{i C}\right), \\
K_{1 i} & =K_{1}\left(S_{i T}, S_{i C}\right), \\
K_{2 i} & =K_{2}\left(S_{i T}, S_{i C}\right), \\
K_{212 i} & =K_{212}\left(S_{i T}, S_{i C}\right), \\
K_{211 i} & =K_{211}\left(S_{i T}, S_{i C}\right), \\
\delta_{i A} & =1-\delta_{i T}-\delta_{i C},
\end{aligned}
$$

$$
\begin{aligned}
\frac{\partial}{\partial \phi_{r}} H_{1 i} & =\frac{\partial}{\partial \phi_{r}}\left(\delta_{i T} \frac{K_{11 i}}{K_{1 i}}+\delta_{i C} \frac{K_{12 i}}{K_{2 i}}+\delta_{i A} \frac{K_{1 i}}{K_{i}}\right) \\
& =\delta_{i T} \frac{K_{211 i} K_{1}-K_{11 i} K_{21 i}}{K_{1 i}^{2}} \frac{\partial}{\partial \phi_{r}} S_{i C}+\delta_{i C} \frac{K_{212 i} K_{2 i}-K_{12 i} K_{22 i}}{K_{2 i}^{2}} \frac{\partial}{\partial \phi_{r}} S_{i C}+\delta_{i A} \frac{K_{21 i} K_{i}-K_{1 i} K_{2 i}}{K_{i}^{2}} \frac{\partial}{\partial \phi_{r}} S_{i C} \\
& =\left(\delta_{i T} \frac{K_{211 i} K_{1}-K_{11 i} K_{21 i}}{K_{1 i}^{2}}+\delta_{i C} \frac{K_{212 i} K_{2 i}-K_{12 i} K_{22 i}}{K_{2 i}^{2}}+\delta_{i A} \frac{K_{21 i} K_{i}-K_{1 i} K_{2 i}}{K_{i}^{2}}\right) \frac{\partial}{\partial \phi_{r}} S_{i C}
\end{aligned}
$$

agora, reescrevendo

$$
\frac{\partial}{\partial S_{i C}} H_{1 i}=\delta_{i T} \frac{K_{211 i} K_{1}-K_{11 i} K_{21 i}}{K_{1 i}^{2}}+\delta_{i C} \frac{K_{212 i} K_{2 i}-K_{12 i} K_{22 i}}{K_{2 i}^{2}}+\delta_{i A} \frac{K_{21 i} K_{i}-K_{1 i} K_{2 i}}{K_{i}^{2}}
$$

fica

$$
\frac{\partial}{\partial \phi_{r}} H_{1 i}=\frac{\partial}{\partial S_{i C}} H_{1 i} \frac{\partial}{\partial \phi_{r}} S_{i C}=-Z_{i t} \Lambda_{i C} S_{i C} \frac{\partial}{\partial S_{i C}} H_{1 i} .
$$


Logo,

$$
\begin{aligned}
\frac{\partial^{2}}{\partial \phi_{r} \beta_{j}} \Phi(\eta) & =\sum_{i=1}^{n}-\left(-Z_{i r} \Lambda_{i T} S_{i T} \Lambda_{i C} S_{i C} \frac{\partial}{\partial S_{i C}} H_{1 i}\right) Z_{i j} \\
& =\sum_{i=1}^{n} \Lambda_{i T} S_{i T} \Lambda_{i C} S_{i C} \frac{\partial}{\partial S_{i C}} H_{1 i} Z_{i r} Z_{i j} .
\end{aligned}
$$

Como

$$
\frac{\partial^{2}}{\partial \beta_{r} \phi_{j}} \Phi(\eta)=\frac{\partial^{2}}{\partial \phi_{r} \beta_{j}} \Phi(\eta)
$$

logo a matriz hessiana dos coeficientes de regressão $\beta$ e $\phi$ é

$$
S_{1}=\left[\begin{array}{cc}
\frac{\partial^{2}}{\partial \beta^{\top} \partial \boldsymbol{\beta}} \Phi(\eta) & \frac{\partial^{2}}{\partial \beta^{\top} \partial \phi} \Phi(\eta) \\
\frac{\partial^{2}}{\partial \phi^{\top} \partial \boldsymbol{\beta}} \Phi(\eta) & \frac{\partial^{2}}{\partial \phi^{\top} \partial \phi} \Phi(\eta)
\end{array}\right] .
$$

\section{Matriz Hessiana $S$ com Basais Paramétricas}

Essa subseção é destinada ao desenvolvimento das componentes da ma modelo de Xu et al. (2018) com as seguintes funções basais definidas

$$
\left\{\begin{array}{l}
\lambda_{0 T}(x)=\frac{\alpha_{t} x^{\alpha_{t}-1}}{\lambda_{t}} \\
\lambda_{0 C}(x)=\frac{\alpha_{c} x^{\alpha_{c}-1}}{\lambda_{c}}
\end{array} .\right.
$$

A seguir, as derivadas das componentes da função de log-verossimilhança (3.13) com relação ao parâmetro $\alpha_{t}$ :

$$
\begin{gathered}
\frac{\partial}{\partial \alpha_{t}} l_{i T}=\frac{1}{\alpha_{t}}+\log \left(\frac{x_{i}}{\lambda_{t}}\right)+0-\Lambda_{i T} \log \left(\frac{x_{i}}{\lambda_{t}}\right)-\frac{K_{11 i}}{K_{1 i}} S_{i T} \Lambda_{i T} \log \left(\frac{x_{i}}{\lambda_{t}}\right) \\
=\frac{1}{\alpha_{t}}+\left(1-\Lambda_{i T}-\frac{K_{11 i}}{K_{1 i}} S_{i T} \Lambda_{i T}\right) \log \left(\frac{x_{i}}{\lambda_{t}}\right) \\
\frac{\partial}{\partial \alpha_{t}} l_{i C}=-\frac{K_{12 i}}{K_{2 i}} S_{i T} \Lambda_{i T} \log \left(\frac{x_{i}}{\lambda_{t}}\right)
\end{gathered}
$$

e

$$
\frac{\partial}{\partial \alpha_{t}} l_{i A}=-\frac{K_{1 i}}{K_{i}} S_{i T} \Lambda_{i T} \log \left(\frac{x_{i}}{\lambda_{t}}\right)
$$

Então

$$
\frac{\partial}{\partial \alpha_{t}} \Phi(\eta)=\sum_{i=1}^{n} \frac{\delta_{i T}}{\alpha_{t}}+\left(\delta_{i T}-\Lambda_{i T} \delta_{i T}-H_{1 i} S_{i T} \Lambda_{i T}\right) \log \left(\frac{x_{i}}{\lambda_{t}}\right) .
$$

Analogamente, as derivadas das componentes da função de log-verossimilhança (3.13) com 
relação ao parâmetro $\alpha_{c}$ resultam:

$$
\frac{\partial}{\partial \alpha_{c}} \Phi(\eta)=\sum_{i=1}^{n} \frac{\delta_{i C}}{\alpha_{\mathcal{C}}}+\left(\delta_{i C}-\Lambda_{i C} \delta_{i C}-H_{2 i} S_{i C} \Lambda_{i C}\right) \log \left(\frac{x_{i}}{\lambda_{c}}\right) .
$$

as derivadas das componentes da função de log-verossimilhança (3.13) com relação ao parâmetro $\alpha_{c}$ resultam:

$$
\begin{aligned}
\frac{\partial}{\partial \lambda_{t}} l_{i T} & =-\frac{\alpha_{t}}{\lambda_{t}}+\frac{\alpha_{t}}{\lambda_{t}} \Lambda_{i T}+\frac{K_{11 i}}{K_{1 i}} S_{i T} \Lambda_{i T} \frac{\alpha_{t}}{\lambda_{t}}, \\
\frac{\partial}{\partial \lambda_{t}} l_{i C} & =\frac{K_{12 i}}{K_{2 i}} S_{i T} \Lambda_{i T} \frac{\alpha_{t}}{\lambda_{t}}, \\
\frac{\partial}{\partial \lambda_{t}} l_{i A} & =\frac{K_{1 i}}{K_{i}} S_{i T} \Lambda_{i T} \frac{\alpha_{t}}{\lambda_{t}} .
\end{aligned}
$$

Logo

$$
\frac{\partial}{\partial \lambda_{t}} \Phi(\eta)=\sum_{i=1}^{n}-\frac{\alpha_{t}}{\lambda_{t}} \delta_{i T}+\left(\Lambda_{i T} \delta_{i T}+H_{1 i} S_{i T} \Lambda_{i T}\right) \frac{\alpha_{t}}{\lambda_{t}} .
$$

Analogamente,

$$
\frac{\partial}{\partial \lambda_{c}} \Phi(\eta)=\sum_{i=1}^{n}-\frac{\alpha_{c}}{\lambda_{c}} \delta_{i C}+\left(\Lambda_{i C} \delta_{i C}+H_{2 i} S_{i C} \Lambda_{i C}\right) \frac{\alpha_{c}}{\lambda_{c}}
$$

A seguir, as derivadas de segunda ordem das componentes da função de log-verossimilhança (3.13) com relação a $\alpha_{t}$ são:

$$
\begin{aligned}
\frac{\partial^{2}}{\partial \alpha_{t}^{2}} \Phi(\eta) & =\frac{\partial}{\partial \alpha_{t}}\left\{\sum_{i=1}^{n} \frac{\delta_{i T}}{\alpha_{t}}+\left[\delta_{i T}-\Lambda_{i T}\left(\delta_{i T}+H_{1 i} S_{i T}\right)\right] \log \left(\frac{x_{i}}{\lambda_{t}}\right)\right\} \\
& =\sum_{i=1}^{n}-\frac{\delta_{i T}}{\alpha_{t}^{2}}-\left[\left(\delta_{i T}+H_{1 i} S_{i T}\right) \frac{\partial}{\partial \alpha_{t}} \Lambda_{i T}+\Lambda_{i T} \frac{\partial}{\partial \alpha_{t}}\left(\delta_{i T}+H_{1 i} S_{i T}\right)\right]
\end{aligned}
$$

Mas sejam

$$
\begin{aligned}
a_{1} & =\left(\delta_{i T}+H_{1 i} S_{i T}\right) \frac{\partial}{\partial \alpha_{t}} \Lambda_{i T} \\
& =\frac{1}{\alpha_{t}}\left(\delta_{i T} \Lambda_{i T}+H_{1 i} S_{i T} \Lambda_{i T}\right) \log \Lambda_{0 i T},
\end{aligned}
$$

e

$$
\begin{aligned}
a_{2} & =\Lambda_{i T} \frac{\partial}{\partial \alpha_{t}}\left(\delta_{i T}+H_{1 i} S_{i T}\right) \\
& =-\frac{1}{\alpha_{t}}\left(S_{i T}^{2} \Lambda_{i T}^{2} \frac{\partial}{\partial S_{i T}} H_{1 i}+H_{1 i} S_{i T} \Lambda_{i T}^{2}\right) \log \Lambda_{0 i T},
\end{aligned}
$$


então

$$
\frac{\partial^{2}}{\partial \alpha_{t}^{2}} \Phi(\eta)=\sum_{i=1}^{n}-\frac{\delta_{i T}}{\alpha_{t}^{2}}-\left(a_{1}+a_{2}\right) \log \left(\frac{x_{i}}{\lambda_{t}}\right) .
$$

Similarmente,

$$
\frac{\partial^{2}}{\partial \alpha_{c}^{2}} \Phi(\eta)=\sum_{i=1}^{n}-\frac{\delta_{i C}}{\alpha_{c}^{2}}-\left(b_{1}+b_{2}\right) \log \left(\frac{x_{i}}{\lambda_{c}}\right)
$$

sendo que

$$
\begin{aligned}
b_{1} & =\left(\delta_{i C}+H_{2 i} S_{i C}\right) \frac{\partial}{\partial \alpha_{c}} \Lambda_{i C} \\
& =\frac{1}{\alpha_{c}}\left(\delta_{i C} \Lambda_{i C}+H_{2 i} S_{i C} \Lambda_{i C}\right) \log \Lambda_{0 i C},
\end{aligned}
$$

$\mathrm{e}$

$$
\begin{aligned}
b_{2} & =\Lambda_{i C} \frac{\partial}{\partial \alpha_{c}}\left(\delta_{i C}+H_{2 i} S_{i C}\right) \\
& =-\frac{1}{\alpha_{c}}\left(S_{i C}^{2} \Lambda_{i C}^{2} \frac{\partial}{\partial S_{i C}} H_{2 i}+H_{2 i} S_{i C} \Lambda_{i C}^{2}\right) \log \Lambda_{0 i C} .
\end{aligned}
$$

A seguir, depois de alguns cálculos, a derivada de segunda ordem da função de logverossimilhança (3.13) com relação ao parâmetro $\lambda_{t}$ :

$$
\frac{\partial^{2}}{\partial \lambda_{t}^{2}} \Phi(\eta)=\sum_{i=1}^{n} \frac{\alpha_{t}}{\lambda_{t}}\left[\delta_{i T}-\left(A_{1}-B_{1}\right)\right],
$$

sendo

$$
A_{1}=\left(\delta_{i T}-\Lambda_{i T}\left(\delta_{i T}+H_{1 i} S_{i T}\right)\right) \log \frac{x_{i}}{\lambda_{t}}
$$

$\mathrm{e}$

$$
B_{1}=\alpha_{t}\left[S_{i T} \Lambda_{i T}^{2}\left(S_{i T} \frac{\partial}{\partial S_{i T}} H_{1 i}+H_{1 i}\right)-\left(\Lambda_{i T} \delta_{i T}+H_{1 i} S_{i T} \Lambda_{i T}\right)\right] .
$$

A seguir serão apresentados mais outros resultados sem demonstração:

$$
\begin{gathered}
\frac{\partial^{2}}{\partial \alpha_{t} \partial \lambda_{t}} \Phi(\eta)=\sum_{i=1}^{n}\left[\Lambda_{i T} \delta_{i T}+H_{1 i} S_{i T} \Lambda_{i T}-S_{i T} \Lambda_{i T}^{2}\left(S_{i T} \frac{\partial}{\partial S_{i T}} H_{1 i}+H_{1 i}\right)\right] \log \frac{x_{i}}{\lambda_{t}} \\
\frac{\partial^{2}}{\partial \lambda_{c} \partial \alpha_{t}} \Phi(\eta)=-\frac{\alpha_{c}}{\alpha_{t} \lambda_{c}} \sum_{i=1}^{n} S_{i T} \Lambda_{i T} S_{i C} \Lambda_{i C} \frac{\partial}{\partial S_{i C}} H_{1 i} \\
\frac{\partial^{2}}{\partial \lambda_{t} \partial \alpha_{c}} \Phi(\eta)=-\frac{\alpha_{t}}{\alpha_{c} \lambda_{t}} \sum_{i=1}^{n} S_{i T} \Lambda_{i T} S_{i C} \Lambda_{i C} \frac{\partial}{\partial S_{i T}} H_{2 i}
\end{gathered}
$$




$$
\begin{gathered}
\frac{\partial}{\partial S_{i T}} H_{2 i}=\frac{\partial}{\partial \lambda_{t}} H_{2 i} . \\
\frac{\partial^{2}}{\partial \alpha_{c} \partial \alpha_{t}} \Phi(\eta)=\frac{1}{\alpha_{t} \alpha_{c}} \sum_{i=1}^{n} S_{i T} \Lambda_{i T} S_{i C} \Lambda_{i C} \Lambda_{i 0 C} \frac{\partial}{\partial S_{i C}} H_{1 i} \\
\frac{\partial^{2}}{\partial \lambda_{c} \partial \lambda_{t}} \Phi(\eta)=-\frac{\alpha_{c} \alpha_{t}}{\lambda_{c} \lambda_{t}} \sum_{i=1}^{n} S_{i T} \Lambda_{i T} S_{i C} \Lambda_{i C} \frac{\partial}{\partial S_{i C}} H_{1 i} .
\end{gathered}
$$

A seguir, são apresentadas as componentes

$$
\begin{aligned}
& \left\{\begin{array}{l}
\frac{\partial^{2}}{\partial \beta_{j} \partial \alpha_{t}} \Phi(\eta) \\
\frac{\partial^{2}}{\partial \beta_{j} \partial \alpha_{c}} \Phi(\eta) \\
\frac{\partial^{2}}{\partial \phi_{j} \partial \lambda_{t}} \Phi(\eta) \\
\frac{\partial^{2}}{\partial \phi_{j} \partial \lambda_{c}} \Phi(\eta)
\end{array}\right. \\
& \frac{\partial^{2}}{\partial \beta_{j} \partial \alpha_{t}} \Phi(\boldsymbol{\eta})=-\sum_{i=1}^{n}\left(\delta_{i T}+S_{i T} \frac{\partial}{\partial S_{i T}} H_{1 i}+H_{1 i} S_{i T}-\Lambda_{i T} S_{i T} H_{1 i}\right) Z_{i j} \Lambda_{i T} \log \frac{x_{i}}{\lambda_{t}} \\
& \frac{\partial^{2}}{\partial \phi_{j} \partial \alpha_{c}} \Phi(\eta)=-\sum_{i=1}^{n}\left(\delta_{i C}+S_{i C} \frac{\partial}{\partial S_{i C}} H_{2 i}+H_{2 i} S_{i C}-\Lambda_{i C} S_{i C} H_{2 i}\right) Z_{i j} \Lambda_{i C} \log \frac{x_{i}}{\lambda_{c}} \\
& \frac{\partial^{2}}{\partial \beta_{j} \partial \alpha_{c}} \Phi(\eta)=\sum_{i=1}^{n}\left(Z_{i j} \Lambda_{i T} \Lambda_{i C} S_{i T} S_{i C} \log \frac{x_{i}}{\lambda_{c}}\right) \frac{\partial}{\partial S_{i T}} H_{2 i} \\
& \frac{\partial^{2}}{\partial \phi_{j} \partial \alpha_{t}} \Phi(\eta)=\sum_{i=1}^{n}\left(Z_{i j} \Lambda_{i T} \Lambda_{i C} S_{i T} S_{i C} \log \frac{x_{i}}{\lambda_{t}}\right) \frac{\partial}{\partial S_{i C}} H_{1 i} \\
& \frac{\partial^{2}}{\partial \beta_{j} \partial \lambda_{t}} \Phi(\eta)=\sum_{i=1}^{n}\left(\delta_{i T}-S_{i T}^{2} \Lambda_{i T} \frac{\partial}{\partial S_{i T}} H_{1 i}\right) Z_{i j} \Lambda_{i T} \frac{\alpha_{t}}{\lambda_{t}} \\
& \frac{\partial^{2}}{\partial \phi_{j} \partial \lambda_{c}} \Phi(\eta)=\sum_{i=1}^{n}\left(\delta_{i C}-S_{i C}^{2} \Lambda_{i C} \frac{\partial}{\partial S_{i C}} H_{2 i}\right) Z_{i j} \Lambda_{i C} \frac{\alpha_{c}}{\lambda_{c}}
\end{aligned}
$$




$$
\begin{gathered}
\frac{\partial^{2}}{\partial \beta_{j} \partial \lambda_{c}} \Phi(\eta)=-\sum_{i=1}^{n} \frac{\alpha_{c}}{\lambda_{c}} Z_{i j} S_{i C} \Lambda_{i C} S_{i T} \Lambda_{i T} \frac{\partial}{\partial S_{i T}} H_{2 i} . \\
\frac{\partial^{2}}{\partial \phi_{j} \partial \lambda_{t}} \Phi(\eta)=-\sum_{i=1}^{n} \frac{\alpha_{t}}{\lambda_{t}} Z_{i j} S_{i C} \Lambda_{i C} S_{i T} \Lambda_{i T} \frac{\partial}{\partial S_{i C}} H_{1 i} .
\end{gathered}
$$

\section{B.2 Modelo Huang e Zhang (2008)}

Esta seção é destinada para apresentar os principais resultados do modelo de Huang e Zhang (2008) discutido nos capítulos 3 e 4. Primeiro é apresentado o gradiente e em seguida a matriz hessiana.

Seja $m=j$ ou $m=j-1$, então

$$
\begin{aligned}
P_{m i} & =\frac{K_{2}\left(S_{T}\left(x_{m}\right), S_{C}\left(x_{i}\right)\right)}{K_{2}\left(S_{T}\left(x_{i}\right), S_{C}\left(x_{i}\right)\right)} \\
& =\frac{K_{2 m i}}{K_{2 i i}},
\end{aligned}
$$

então

$$
\frac{\partial}{\partial \beta_{u}} P_{m i}=\frac{K_{2 i i} K_{12 m i}}{K_{2 i i}^{2}} \frac{\partial}{\partial \beta_{u}} S_{T}\left(x_{m}\right)-\frac{K_{2 m i} K_{12 i i}}{K_{2 i i}^{2}} \frac{\partial}{\partial \beta_{u}} S_{T}\left(x_{i}\right) .
$$

Sejam

$$
\begin{aligned}
& C_{1}=\frac{K_{2 i i} K_{12 m i}}{K_{2 i i}^{2}} \\
& C_{2}=\frac{K_{2 m i} K_{12 i i}}{K_{2 i i}^{2}}
\end{aligned}
$$

então

$$
\begin{aligned}
\frac{\partial}{\partial \beta_{u}} C_{1} & =\frac{\left(K_{12 m i} K_{12 i i} a_{i}+K_{2 i i} K_{112 m i} a_{m}\right)\left(K_{2 i i}\right)^{2}-2\left(K_{2 i i} K_{12 m i}\right) K_{2 i i} K_{12 i i} a_{i}}{K_{2 i i}^{4}} \\
\frac{\partial}{\partial \beta_{u}} C_{2} & =\frac{\left(K_{12 i i} K_{12 m i} a_{m}+K_{2 m i} K_{112 i i} a_{i}\right)\left(K_{2 i i}\right)^{2}-2\left(K_{2 m i} K_{12 m i}\right) K_{2 i i} K_{12 i i} a_{i}}{K_{2 i i}^{4}} .
\end{aligned}
$$

O que implica

$$
\frac{\partial^{2}}{\partial \beta_{u}^{2}} P_{m i}=a_{m} \frac{\partial}{\partial \beta_{u}} C_{1}+C_{1} \frac{\partial^{2}}{\partial \beta_{u}^{2}} S_{T}\left(x_{m}\right)-\left(a_{i} \frac{\partial}{\partial \beta_{u}} C_{2}+C_{2} \frac{\partial^{2}}{\partial \beta_{u}^{2}} S_{T}\left(x_{i}\right)\right),
$$


sendo

$$
\begin{aligned}
a_{i} & =\frac{\partial}{\partial \beta_{u}} S_{T}\left(x_{i}\right), \\
a_{m} & =\frac{\partial}{\partial \beta_{u}} S_{T}\left(x_{m}\right) .
\end{aligned}
$$

Agora sejam

$$
\begin{aligned}
\frac{\partial}{\partial \phi_{r}} C_{1} & =\frac{K_{212 m i} K_{2 i i} b_{m}-K_{12 m i} K_{22 i i} b_{i}}{\left(K_{2 i i}\right)^{2}} \\
\frac{\partial}{\partial \phi_{r}} C_{2} & =\frac{K_{22 m i} K_{12 i i} b_{m}+K_{2 m i} K_{212 i i} b_{i}}{\left(K_{2 i i}\right)^{2}}-\frac{2 K_{12 i i} K_{2 m i} K_{22 i i} b_{i}}{\left(K_{2 i i}\right)^{3}}
\end{aligned}
$$

que implica

$$
\frac{\partial^{2}}{\partial \phi_{r} \partial \beta_{u}} P_{m i}=a_{m} \frac{\partial}{\partial \phi_{r}} C_{1}-a_{i} \frac{\partial}{\partial \phi_{r}} C_{2}
$$

Agora

$$
\frac{\partial}{\partial \phi_{r}} P_{m i}=b_{m} E_{1}-b_{i} E_{2}
$$

sendo que

$$
\begin{aligned}
& E_{1}=\frac{K_{2 i i} K_{22 m i}}{\left(K_{2 i i}\right)^{2}}, \\
& E_{2}=\frac{K_{2 m i} K_{12 i i}}{\left(K_{2 i i}\right)^{2}} .
\end{aligned}
$$

Implica que

$$
\frac{\partial^{2}}{\partial \phi_{r}^{2}} P_{m i}=\left(\frac{\partial}{\partial \phi_{r}} b_{m}\right) E_{1}+b_{m} \frac{\partial}{\partial \phi_{r}} E_{1}-\left[\left(\frac{\partial}{\partial \phi_{r}} b_{i}\right) E_{2}+b_{i} \frac{\partial}{\partial \phi_{r}} E_{2}\right],
$$

Quanto as derivadas da função

$$
Q_{m i}=\frac{K_{1 m i}}{K_{1 i i}}
$$

elas são semelhantes as derivadas anteriores de $P_{m i}$ diferenciando apenas na ordem da cópula, isto é, $K_{1 \sim}$ e agora as derivadas são das funções de sobrevivência, $S_{C}(x)$, do tempo de censura. Então ficou decidido omiti-las.

Agora seja

$$
\frac{\partial}{\partial \beta_{u}} l_{T}(\boldsymbol{\beta})=\frac{\partial}{\partial \beta_{u}} A_{1}(\boldsymbol{\beta})+\frac{\partial}{\partial \beta_{u}} B_{1}(\boldsymbol{\beta})
$$

em que

$$
\frac{\partial}{\partial \beta_{u}} A_{1}(\beta)=\sum_{j=1}^{n} \sum_{i=1}^{j}\left(\log P_{j i}\right) \frac{\partial}{\partial \beta_{u}} D_{j i}+D_{j i} \frac{\frac{\partial}{\partial \beta_{u}} P j i}{P_{j i}}+Z_{i}^{\top} \beta \frac{\partial}{\partial \beta_{u}} D_{j i}+D_{j i} Z_{i u}
$$


e

$$
\frac{\partial}{\partial \beta_{u}} B_{1}(\beta)=\sum_{j=1}^{n} \sum_{i=1}^{j} S_{k} \frac{\partial}{\partial \beta_{u}} D_{j i}+D_{j i} \frac{\partial}{\partial \beta_{u}} S_{k}
$$

sendo que

$$
S_{k}=\log \left(\sum_{k=1}^{n} P_{j k} e^{Z_{k}^{\top} \boldsymbol{\beta}}\right) \Longrightarrow \frac{\partial}{\partial \beta_{u}} S_{k}=\frac{\sum_{k=1}^{n} e^{\mathbf{Z}_{k}^{\top} \boldsymbol{\beta}} \frac{\partial}{\partial \beta_{u}} P_{j k}+Z_{k u} P_{j k]} e^{\mathbf{Z}_{k}^{\top} \boldsymbol{\beta}}}{\sum_{k=1}^{n} P_{j k} e_{k}^{\mathbf{Z}_{k}^{\top} \boldsymbol{\beta}}} .
$$

Agora seja

$$
\frac{\partial}{\partial \beta_{u}} l_{C}(\phi)=\frac{\partial}{\partial \beta_{u}} A_{2}(\beta)+\frac{\partial}{\partial \beta_{u}} B_{2}(\beta)
$$

em que

$$
\frac{\partial}{\partial \beta_{u}} A_{2}(\beta)=\sum_{j=1}^{n} \sum_{i=1}^{j}\left(\log Q_{j i}\right) \frac{\partial}{\partial \beta_{u}} U_{j i}+U_{j i} \frac{\frac{\partial}{\partial \beta_{u}} Q j i}{Q_{j i}}+Z_{i}^{\top} \beta \frac{\partial}{\partial \beta_{u}} U_{j i}+U_{j i} Z_{i u}
$$

e

$$
\frac{\partial}{\partial \beta_{u}} B_{2}(\boldsymbol{\beta})=\sum_{j=1}^{n} \sum_{i=1}^{j} H_{k} \frac{\partial}{\partial \beta_{u}} U_{j i}+U_{j i} \frac{\partial}{\partial \beta_{u}} H_{k}
$$

sendo que

$$
H_{k}=\log \left(\sum_{k=1}^{n} Q_{j k} e_{k}^{Z_{k}^{\top} \beta}\right) \Longrightarrow \frac{\partial}{\partial \beta_{u}} H_{k}=\frac{\sum_{k=1}^{n} e^{Z_{k}^{\top} \boldsymbol{\beta}} \frac{\partial}{\partial \beta_{u}} Q_{j k}+Z_{k u} Q_{j k]} e^{Z_{k}^{\top} \boldsymbol{\beta}}}{\sum_{k=1}^{n} Q_{j k} e_{k}^{Z_{k} \beta}}
$$

Agora seja

$$
\frac{\partial}{\partial \phi_{u}} l_{T}(\boldsymbol{\beta})=\frac{\partial}{\partial \phi_{u}} A_{1}(\boldsymbol{\beta})+\frac{\partial}{\partial \phi_{u}} B_{1}(\boldsymbol{\beta})
$$

em que

$$
\frac{\partial}{\partial \phi_{u}} A_{1}(\boldsymbol{\beta})=\sum_{j=1}^{n} \sum_{i=1}^{j}\left(\log P_{j i}\right) \frac{\partial}{\partial \phi_{u}} D_{j i}+D_{j i} \frac{\frac{\partial}{\partial \phi_{u}} P j i}{P_{j i}}+Z_{i}^{\top} \boldsymbol{\phi} \frac{\partial}{\partial \phi_{u}} D_{j i}+D_{j i} Z_{i u}
$$

e

$$
\frac{\partial}{\partial \phi_{u}} B_{1}(\boldsymbol{\beta})=\sum_{j=1}^{n} \sum_{i=1}^{j} S_{k} \frac{\partial}{\partial \phi_{u}} D_{j i}+D_{j i} \frac{\partial}{\partial \phi_{u}} S_{k},
$$


sendo que

$$
S_{k}=\log \left(\sum_{k=1}^{n} P_{j k} e^{Z_{k}^{\top} \boldsymbol{\phi}}\right) \Longrightarrow \frac{\partial}{\partial \phi_{u}} S_{k}=\frac{\sum_{k=1}^{n} e^{\mathbf{Z}_{k}^{\top} \boldsymbol{\phi}} \frac{\partial}{\partial \phi_{u}} P_{j k}+Z_{k u} P_{j k]} e^{\mathbf{Z}_{k}^{\top} \boldsymbol{\phi}}}{\sum_{k=1}^{n} P_{j k} e_{k}^{\mathbf{Z}_{k} \boldsymbol{\phi}}}
$$




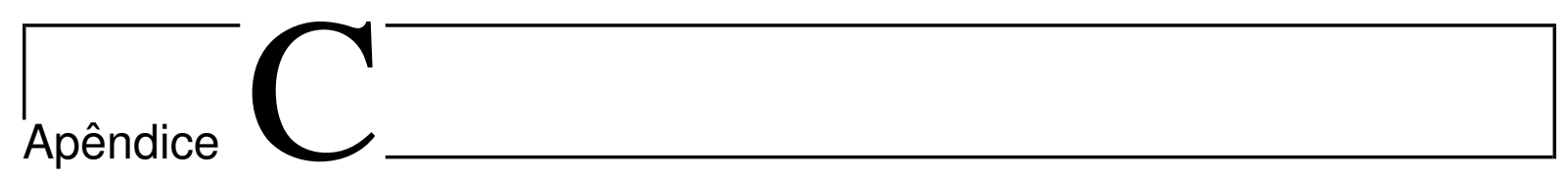

\section{Tabelas}

As tabelas C.1 a C.12 são as tabulações resumidas do estudo de simulação discutido na seção 4.1. As tabelas C.13 a C.16 são as tabulações resumidas do estudo de simulação discutido na seção 4.2 .

Tabela C.1: Estimativas das Réplicas $n=300$ pelo Modelo Paramétrico (Clayton)

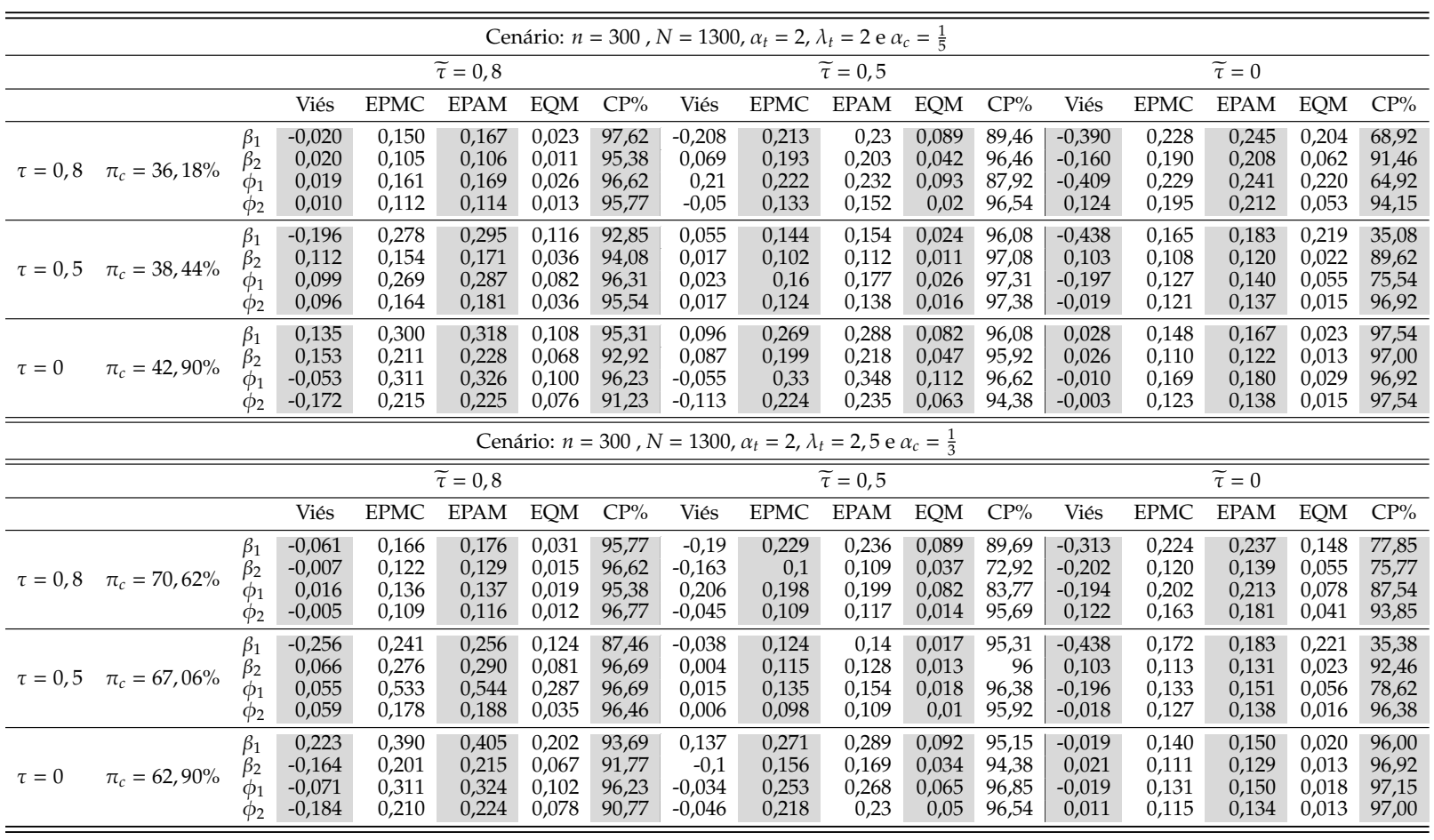


Tabela C.2: Estimativas das Réplicas $n=1000$ pelo Modelo Paramétrico (Clayton)

\begin{tabular}{|c|c|c|c|c|c|c|c|c|c|c|c|c|c|c|c|c|c|}
\hline \multicolumn{18}{|c|}{ Cenário: $n=300, N=1300, \alpha_{t}=2, \lambda_{t}=2$ e $\alpha_{c}=\frac{1}{5}$} \\
\hline & & & \multicolumn{5}{|c|}{$\tilde{\tau}=0,8$} & \multicolumn{5}{|c|}{$\widetilde{\tau}=0,5$} & \multicolumn{5}{|c|}{$\tilde{\tau}=0$} \\
\hline & & & Viés & EPMC & EPAM & EQM & $\mathrm{CP} \%$ & Viés & EPMC & EPAM & EQM & $\mathrm{CP} \%$ & Viés & EPMC & EPAM & EQM & $\mathrm{CP} \%$ \\
\hline$\tau=0,8$ & $\pi_{c}=36,18 \%$ & $\begin{array}{l}\beta_{1} \\
\beta_{2} \\
\phi_{1} \\
\phi_{2} \\
\end{array}$ & $\begin{array}{r}-0,001 \\
0,002 \\
-0,003 \\
0,002 \\
\end{array}$ & $\begin{array}{l}0,130 \\
0,088 \\
0,138 \\
0,102 \\
\end{array}$ & $\begin{array}{l}0,147 \\
0,089 \\
0,146 \\
0,104 \\
\end{array}$ & $\begin{array}{l}0,017 \\
0,008 \\
0,019 \\
0,010 \\
\end{array}$ & $\begin{array}{l}98,38 \\
95,92 \\
96,85 \\
96,23 \\
\end{array}$ & $\begin{array}{r}-0,151 \\
0,031 \\
0,182 \\
-0,038 \\
\end{array}$ & $\begin{array}{l}0,181 \\
0,148 \\
0,197 \\
0,113 \\
\end{array}$ & $\begin{array}{l}0,194 \\
0,165 \\
0,214 \\
0,131 \\
\end{array}$ & $\begin{array}{l}0,056 \\
0,023 \\
0,072 \\
0,014 \\
\end{array}$ & $\begin{array}{l}91,00 \\
97,38 \\
90,15 \\
97,31 \\
\end{array}$ & $\begin{array}{r}-0,306 \\
-0,115 \\
-0,387 \\
0,089 \\
\end{array}$ & $\begin{array}{l}0,175 \\
0,126 \\
0,182 \\
0,153 \\
\end{array}$ & $\begin{array}{l}0,193 \\
0,139 \\
0,193 \\
0,170 \\
\end{array}$ & $\begin{array}{l}0,124 \\
0,029 \\
0,183 \\
0,031 \\
\end{array}$ & $\begin{array}{l}69,92 \\
90,85 \\
52,77 \\
96,23 \\
\end{array}$ \\
\hline$\tau=0,5$ & $\pi_{c}=38,44 \%$ & $\begin{array}{l}\beta_{1} \\
\beta_{2} \\
\beta_{2} \\
\beta_{2} \\
\end{array}$ & $\begin{array}{r}-0,097 \\
0,052 \\
0,035 \\
0,046 \\
\end{array}$ & $\begin{array}{l}0,179 \\
0,104 \\
0,250 \\
0,134 \\
\end{array}$ & $\begin{array}{l}0,189 \\
0,114 \\
0,268 \\
0,148 \\
\end{array}$ & $\begin{array}{l}0,041 \\
0,014 \\
0,064 \\
0,020 \\
\end{array}$ & $\begin{array}{l}94,62 \\
95,85 \\
97,23 \\
97,00 \\
\end{array}$ & $\begin{array}{r}-0,002 \\
-0,001 \\
0,011 \\
-0,002 \\
\end{array}$ & $\begin{array}{l}0,083 \\
0,067 \\
0,135 \\
0,097\end{array}$ & $\begin{array}{l}0,096 \\
0,084 \\
0,152 \\
0,109\end{array}$ & $\begin{array}{l}0,007 \\
0,004 \\
0,018 \\
0,009 \\
\end{array}$ & $\begin{array}{l}98,38 \\
98,92 \\
97,62 \\
97,77 \\
\end{array}$ & $\begin{array}{r}-0,202 \\
0,036 \\
0,011 \\
0,080 \\
\end{array}$ & $\begin{array}{l}0,237 \\
0,097 \\
0,191 \\
0,137\end{array}$ & $\begin{array}{l}0,255 \\
0,112 \\
0,206 \\
0,149 \\
\end{array}$ & $\begin{array}{l}0,097 \\
0,011 \\
0,037 \\
0,025 \\
\end{array}$ & $\begin{array}{l}91,92 \\
97,38 \\
97,23 \\
94,69 \\
\end{array}$ \\
\hline-0 & $42,90 \%$ & $\begin{array}{l}\beta_{1} \\
\beta_{2} \\
\phi_{1} \\
\phi_{2}\end{array}$ & $\begin{array}{r}0,044 \\
0,071 \\
-0,036 \\
-0,114\end{array}$ & $\begin{array}{l}0,299 \\
0,180 \\
0,310 \\
0,214\end{array}$ & $\begin{array}{l}0,302 \\
0,182 \\
0,316 \\
0,221\end{array}$ & $\begin{array}{l}0,091 \\
0,037 \\
0,097 \\
0,059\end{array}$ & $\begin{array}{l}96,08 \\
94,31 \\
96,38 \\
94,23\end{array}$ & $\begin{array}{r}0,030 \\
-0,050 \\
-0,050 \\
-0,110\end{array}$ & $\begin{array}{l}0,218 \\
0,108 \\
0,298 \\
0,199\end{array}$ & $\begin{array}{l}0,228 \\
0,119 \\
0,308 \\
0,216\end{array}$ & $\begin{array}{l}0,048 \\
0,014 \\
0,091 \\
0,052\end{array}$ & $\begin{array}{l}96,00 \\
96,23 \\
96,54 \\
95,54\end{array}$ & $\begin{array}{r}0,003 \\
0,002 \\
-0,001 \\
0,000\end{array}$ & $\begin{array}{l}0,081 \\
0,100 \\
0,090 \\
0,066\end{array}$ & $\begin{array}{l}0,100 \\
0,110 \\
0,108 \\
0,085\end{array}$ & $\begin{array}{l}0,007 \\
0,010 \\
0,008 \\
0,004\end{array}$ & $\begin{array}{l}98,77 \\
97,62 \\
98,54 \\
99,23\end{array}$ \\
\hline \multicolumn{18}{|c|}{ Cenário: $n=300, N=1300, \alpha_{t}=2, \lambda_{t}=2,5$ e $\alpha_{c}=\frac{1}{3}$} \\
\hline & & & \multicolumn{5}{|c|}{$\tilde{\tau}=0,8$} & \multicolumn{5}{|c|}{$\tilde{\tau}=0,5$} & \multicolumn{5}{|c|}{$\tilde{\tau}=0$} \\
\hline & & & Viés & EPMC & EPAM & EQM & $\mathrm{CP} \%$ & Viés & EPMC & EPAM & EQM & $\mathrm{CP} \%$ & Viés & EPMC & EPAM & EQM & $\mathrm{CP} \%$ \\
\hline$\tau=0,8$ & $\pi_{\mathcal{C}}=70,62 \%$ & $\begin{array}{l}\beta_{1} \\
\beta_{2} \\
\phi_{1} \\
\phi_{2}\end{array}$ & $\begin{array}{r}0,002 \\
0,002 \\
-0,003 \\
0,005\end{array}$ & $\begin{array}{l}0,150 \\
0,121 \\
0,109 \\
0,099\end{array}$ & $\begin{array}{l}0,156 \\
0,137 \\
0,110 \\
0,115\end{array}$ & $\begin{array}{l}0,023 \\
0,015 \\
0,012 \\
0,010\end{array}$ & $\begin{array}{l}96,38 \\
97,69 \\
95,77 \\
98,00\end{array}$ & $\begin{array}{r}-0,177 \\
-0,026 \\
0,170 \\
-0,028\end{array}$ & $\begin{array}{l}0,203 \\
0,125 \\
0,200 \\
0,104\end{array}$ & $\begin{array}{l}0,214 \\
0,140 \\
0,213 \\
0,120\end{array}$ & $\begin{array}{l}0,073 \\
0,016 \\
0,069 \\
0,012\end{array}$ & $\begin{array}{l}90,08 \\
97,31 \\
91,15 \\
97,69\end{array}$ & $\begin{array}{r}-0,295 \\
-0,091 \\
-0,184 \\
0,087\end{array}$ & $\begin{array}{l}0,188 \\
0,100 \\
0,172 \\
0,131\end{array}$ & $\begin{array}{l}0,206 \\
0,115 \\
0,187 \\
0,145\end{array}$ & $\begin{array}{l}0,122 \\
0,018 \\
0,063 \\
0,025\end{array}$ & $\begin{array}{l}75,62 \\
93,31 \\
87,46 \\
93,85\end{array}$ \\
\hline$\tau=0,5$ & $\pi_{c}=67,06 \%$ & $\begin{array}{l}\beta_{1} \\
\beta_{2} \\
\phi_{1} \\
\phi_{2}\end{array}$ & $\begin{array}{r}-0,195 \\
0,033 \\
0,047 \\
0,023 \\
\end{array}$ & $\begin{array}{l}0,232 \\
0,166 \\
0,233 \\
0,116\end{array}$ & $\begin{array}{l}0,243 \\
0,180 \\
0,244 \\
0,133 \\
\end{array}$ & $\begin{array}{l}0,092 \\
0,029 \\
0,056 \\
0,014\end{array}$ & $\begin{array}{l}90,00 \\
96,46 \\
95,92 \\
97,46 \\
\end{array}$ & $\begin{array}{r}-0,001 \\
-0,001 \\
0,000 \\
-0,002\end{array}$ & $\begin{array}{l}0,057 \\
0,058 \\
0,058 \\
0,055\end{array}$ & $\begin{array}{l}0,072 \\
0,072 \\
0,077 \\
0,067\end{array}$ & $\begin{array}{l}0,003 \\
0,003 \\
0,003 \\
0,003 \\
\end{array}$ & $\begin{array}{l}98,92 \\
98,85 \\
99,62 \\
98,62\end{array}$ & $\begin{array}{r}-0,440 \\
0,102 \\
-0,198 \\
-0,020\end{array}$ & $\begin{array}{l}0,110 \\
0,111 \\
0,100 \\
0,120\end{array}$ & $\begin{array}{l}0,117 \\
0,113 \\
0,109 \\
0,125\end{array}$ & $\begin{array}{l}0,206 \\
0,023 \\
0,049 \\
0,015\end{array}$ & $\begin{array}{r}3,38 \\
85,15 \\
59,15 \\
96,00 \\
\end{array}$ \\
\hline$\tau=0$ & $\pi_{c}=62,90 \%$ & $\begin{array}{l}\beta_{1} \\
\beta_{2} \\
\beta_{2} \\
\beta_{2} \\
\end{array}$ & $\begin{array}{r}0,111 \\
0,017 \\
-0,051 \\
-0,124 \\
\end{array}$ & $\begin{array}{l}0,280 \\
0,201 \\
0,311 \\
0,215 \\
\end{array}$ & $\begin{array}{l}0,288 \\
0,210 \\
0,320 \\
0,217 \\
\end{array}$ & $\begin{array}{l}0,091 \\
0,041 \\
0,099 \\
0,062 \\
\end{array}$ & $\begin{array}{l}94,92 \\
96,62 \\
96,00 \\
92,54 \\
\end{array}$ & $\begin{array}{r}0,049 \\
-0,010 \\
-0,051 \\
-0,111 \\
\end{array}$ & $\begin{array}{l}0,266 \\
0,138 \\
0,297 \\
0,198\end{array}$ & $\begin{array}{l}0,285 \\
0,151 \\
0,312 \\
0,216\end{array}$ & $\begin{array}{l}0,073 \\
0,019 \\
0,091 \\
0,052 \\
\end{array}$ & $\begin{array}{l}96,54 \\
97,23 \\
96,46 \\
95,00 \\
\end{array}$ & $\begin{array}{r}-0,002 \\
0,000 \\
0,000 \\
0,000 \\
\end{array}$ & $\begin{array}{l}0,049 \\
0,079 \\
0,089 \\
0,083\end{array}$ & $\begin{array}{l}0,065 \\
0,095 \\
0,102 \\
0,102 \\
\end{array}$ & $\begin{array}{l}0,002 \\
0,006 \\
0,008 \\
0,007 \\
\end{array}$ & $\begin{array}{l}99,31 \\
98,85 \\
98,46 \\
98,92 \\
\end{array}$ \\
\hline
\end{tabular}

Tabela C.3: Estimativas das Réplicas $n=300$ pelo Modelo Paramétrico (Frank)

\begin{tabular}{|c|c|c|c|c|c|c|c|c|c|c|c|c|c|c|c|c|c|}
\hline \multicolumn{18}{|c|}{ Cenário: $n=300, N=1300, \alpha_{t}=2, \lambda_{t}=2$ e $\alpha_{c}=\frac{1}{5}$} \\
\hline & & & \multicolumn{5}{|c|}{$\widetilde{\tau}=0,8$} & \multicolumn{5}{|c|}{$\widetilde{\tau}=0,5$} & \multicolumn{5}{|c|}{$\tilde{\tau}=0$} \\
\hline & & & Viés & EPMC & EPAM & EQM & $\mathrm{CP} \%$ & Viés & EPMC & EPAM & EQM & $\mathrm{CP} \%$ & Viés & EPMC & EPAM & EQM & $\mathrm{CP} \%$ \\
\hline$\tau=0,8$ & $\pi_{c}=36,18 \%$ & $\begin{array}{l}\beta_{1} \\
\beta_{2} \\
\phi_{1} \\
\phi_{2}\end{array}$ & $\begin{array}{r}-0,020 \\
0,014 \\
0,019 \\
0,010\end{array}$ & $\begin{array}{l}0,150 \\
0,105 \\
0,161 \\
0,112\end{array}$ & $\begin{array}{l}0,167 \\
0,106 \\
0,169 \\
0,114\end{array}$ & $\begin{array}{l}0,023 \\
0,011 \\
0,026 \\
0,013\end{array}$ & $\begin{array}{l}97,62 \\
95,62 \\
96,62 \\
95,77\end{array}$ & $\begin{array}{r}-0,204 \\
0,073 \\
0,214 \\
-0,047\end{array}$ & $\begin{array}{l}0,201 \\
0,182 \\
0,209 \\
0,126\end{array}$ & $\begin{array}{l}0,216 \\
0,199 \\
0,221 \\
0,139\end{array}$ & $\begin{array}{l}0,082 \\
0,038 \\
0,089 \\
0,018\end{array}$ & $\begin{array}{l}88,62 \\
96,38 \\
87,08 \\
96,08\end{array}$ & $\begin{array}{r}-0,384 \\
-0,155 \\
-0,403 \\
0,129\end{array}$ & $\begin{array}{l}0,233 \\
0,195 \\
0,234 \\
0,199\end{array}$ & $\begin{array}{l}0,252 \\
0,211 \\
0,248 \\
0,216\end{array}$ & $\begin{array}{l}0,202 \\
0,062 \\
0,217 \\
0,056\end{array}$ & $\begin{array}{l}71,08 \\
92,23 \\
67,15 \\
94,08\end{array}$ \\
\hline$\tau=0,5$ & $\pi_{c}=38,44 \%$ & $\begin{array}{l}\beta_{1} \\
\beta_{2} \\
\phi_{1} \\
\phi_{2}\end{array}$ & $\begin{array}{r}-0,199 \\
0,111 \\
0,096 \\
0,095\end{array}$ & $\begin{array}{l}0,279 \\
0,155 \\
0,270 \\
0,165\end{array}$ & $\begin{array}{l}0,296 \\
0,169 \\
0,285 \\
0,177\end{array}$ & $\begin{array}{l}0,117 \\
0,036 \\
0,082 \\
0,036\end{array}$ & $\begin{array}{l}92,08 \\
93,23 \\
96,15 \\
94,92\end{array}$ & $\begin{array}{r}-0,041 \\
0,005 \\
0,021 \\
0,006\end{array}$ & $\begin{array}{l}0,148 \\
0,112 \\
0,164 \\
0,127\end{array}$ & $\begin{array}{l}0,164 \\
0,122 \\
0,177 \\
0,145\end{array}$ & $\begin{array}{l}0,024 \\
0,013 \\
0,027 \\
0,016\end{array}$ & $\begin{array}{l}97,31 \\
97,54 \\
97,15 \\
97,77\end{array}$ & $\begin{array}{r}-0,753 \\
-0,244 \\
-0,425 \\
0,208\end{array}$ & $\begin{array}{l}0,260 \\
0,203 \\
0,271 \\
0,217\end{array}$ & $\begin{array}{l}0,271 \\
0,213 \\
0,285 \\
0,236\end{array}$ & $\begin{array}{l}0,635 \\
0,101 \\
0,254 \\
0,090\end{array}$ & $\begin{array}{l}22,00 \\
82,54 \\
71,92 \\
89,62\end{array}$ \\
\hline$\tau=0$ & $\pi_{c}=42,90 \%$ & $\begin{array}{l}\beta_{1} \\
\beta_{2} \\
\phi_{1} \\
\phi_{2} \\
\end{array}$ & $\begin{array}{r}0,134 \\
0,122 \\
-0,054 \\
-0,173 \\
\end{array}$ & $\begin{array}{l}0,305 \\
0,214 \\
0,316 \\
0,218\end{array}$ & $\begin{array}{l}0,315 \\
0,226 \\
0,333 \\
0,229\end{array}$ & $\begin{array}{l}0,111 \\
0,061 \\
0,103 \\
0,077\end{array}$ & $\begin{array}{l}95,46 \\
94,08 \\
96,85 \\
90,85 \\
\end{array}$ & $\begin{array}{r}0,100 \\
0,070 \\
-0,050 \\
-0,110 \\
\end{array}$ & $\begin{array}{l}0,308 \\
0,099 \\
0,329 \\
0,224\end{array}$ & $\begin{array}{l}0,323 \\
0,114 \\
0,342 \\
0,237\end{array}$ & $\begin{array}{l}0,105 \\
0,015 \\
0,111 \\
0,062 \\
\end{array}$ & $\begin{array}{l}95,85 \\
94,69 \\
97,00 \\
95,46\end{array}$ & $\begin{array}{r}0,013 \\
0,015 \\
-0,028 \\
-0,016 \\
\end{array}$ & $\begin{array}{l}0,152 \\
0,112 \\
0,173 \\
0,126 \\
\end{array}$ & $\begin{array}{l}0,162 \\
0,122 \\
0,185 \\
0,139 \\
\end{array}$ & $\begin{array}{l}0,023 \\
0,013 \\
0,031 \\
0,016 \\
\end{array}$ & $\begin{array}{l}97,23 \\
97,46 \\
96,92 \\
97,69 \\
\end{array}$ \\
\hline \multicolumn{18}{|c|}{ Cenário: $n=300, N=1300, \alpha_{t}=2, \lambda_{t}=2,5$ e $\alpha_{c}=\frac{1}{3}$} \\
\hline & & & \multicolumn{5}{|c|}{$\bar{\tau}=0,8$} & \multicolumn{5}{|c|}{$\widetilde{\tau}=0,5$} & \multicolumn{5}{|c|}{$\tilde{\tau}=0$} \\
\hline & & & Viés & EPMC & EPAM & EQM & $\mathrm{CP} \%$ & Viés & EPMC & EPAM & EQM & $\mathrm{CP} \%$ & Viés & EPMC & EPAM & EQM & $\mathrm{CP} \%$ \\
\hline$\tau=0,8$ & $\pi_{c}=70,62 \%$ & $\begin{array}{l}\beta_{1} \\
\beta_{2} \\
\phi_{1} \\
\phi_{2} \\
\end{array}$ & $\begin{array}{r}-0,061 \\
-0,007 \\
0,016 \\
-0,005 \\
\end{array}$ & $\begin{array}{l}0,166 \\
0,122 \\
0,136 \\
0,109\end{array}$ & $\begin{array}{l}0,176 \\
0,129 \\
0,137 \\
0,116 \\
\end{array}$ & $\begin{array}{l}0,031 \\
0,015 \\
0,019 \\
0,012 \\
\end{array}$ & $\begin{array}{l}95,77 \\
96,62 \\
95,38 \\
96,77 \\
\end{array}$ & $\begin{array}{r}-0,192 \\
-0,164 \\
0,205 \\
-0,046 \\
\end{array}$ & $\begin{array}{l}0,239 \\
0,105 \\
0,207 \\
0,114 \\
\end{array}$ & $\begin{array}{l}0,248 \\
0,106 \\
0,212 \\
0,124\end{array}$ & $\begin{array}{l}0,094 \\
0,038 \\
0,085 \\
0,015 \\
\end{array}$ & $\begin{array}{l}91,08 \\
69,69 \\
86,77 \\
96,08 \\
\end{array}$ & $\begin{array}{r}-0,307 \\
-0,198 \\
-0,188 \\
0,126 \\
\end{array}$ & $\begin{array}{l}0,234 \\
0,126 \\
0,211 \\
0,170 \\
\end{array}$ & $\begin{array}{l}0,244 \\
0,141 \\
0,225 \\
0,183 \\
\end{array}$ & $\begin{array}{l}0,149 \\
0,055 \\
0,080 \\
0,045 \\
\end{array}$ & $\begin{array}{l}78,92 \\
76,38 \\
90,85 \\
91,31 \\
\end{array}$ \\
\hline$\tau=0,5$ & $\pi_{c}=67,06 \%$ & $\begin{array}{l}\beta_{1} \\
\beta_{2} \\
\beta_{2} \\
\beta_{2}\end{array}$ & $\begin{array}{r}-0,258 \\
0,067 \\
0,057 \\
0,060\end{array}$ & $\begin{array}{l}0,365 \\
0,287 \\
0,553 \\
0,184\end{array}$ & $\begin{array}{l}0,377 \\
0,304 \\
0,564 \\
0,200\end{array}$ & $\begin{array}{l}0,200 \\
0,087 \\
0,309 \\
0,037\end{array}$ & $\begin{array}{l}91,38 \\
96,69 \\
96,23 \\
96,69\end{array}$ & $\begin{array}{r}-0,039 \\
0,003 \\
0,013 \\
0,005\end{array}$ & $\begin{array}{l}0,123 \\
0,114 \\
0,134 \\
0,098\end{array}$ & $\begin{array}{l}0,136 \\
0,128 \\
0,145 \\
0,117\end{array}$ & $\begin{array}{l}0,017 \\
0,013 \\
0,018 \\
0,010\end{array}$ & $\begin{array}{l}96,38 \\
97,31 \\
96,77 \\
97,85\end{array}$ & $\begin{array}{r}-0,437 \\
0,104 \\
-0,195 \\
-0,017\end{array}$ & $\begin{array}{l}0,171 \\
0,111 \\
0,131 \\
0,125\end{array}$ & $\begin{array}{l}0,184 \\
0,122 \\
0,149 \\
0,143 \\
\end{array}$ & $\begin{array}{l}0,220 \\
0,023 \\
0,055 \\
0,016\end{array}$ & $\begin{array}{l}37,23 \\
88,92 \\
77,85 \\
96,23 \\
\end{array}$ \\
\hline $2-0$ & $\pi_{c}=62,90 \%$ & $\begin{array}{l}\beta_{1} \\
\beta_{2} \\
\phi_{1} \\
\phi_{2}\end{array}$ & $\begin{array}{r}0,260 \\
-0,145 \\
-0,043 \\
-0,165\end{array}$ & $\begin{array}{l}0,419 \\
0,196 \\
0,303 \\
0,205\end{array}$ & $\begin{array}{l}0,429 \\
0,209 \\
0,321 \\
0,222\end{array}$ & $\begin{array}{l}0,243 \\
0,059 \\
0,094 \\
0,069\end{array}$ & $\begin{array}{l}92,54 \\
92,00 \\
97,15 \\
91,85\end{array}$ & $\begin{array}{r}0,129 \\
-0,105 \\
-0,042 \\
-0,053\end{array}$ & $\begin{array}{l}0,309 \\
0,161 \\
0,260 \\
0,225\end{array}$ & $\begin{array}{l}0,327 \\
0,175 \\
0,279 \\
0,237\end{array}$ & $\begin{array}{l}0,112 \\
0,037 \\
0,069 \\
0,053\end{array}$ & $\begin{array}{l}95,46 \\
94,00 \\
96,77 \\
96,62\end{array}$ & $\begin{array}{r}0,017 \\
0,018 \\
-0,023 \\
-0,012\end{array}$ & $\begin{array}{l}0,176 \\
0,125 \\
0,156 \\
0,115\end{array}$ & $\begin{array}{l}0,188 \\
0,136 \\
0,174 \\
0,134\end{array}$ & $\begin{array}{l}0,031 \\
0,016 \\
0,025 \\
0,013\end{array}$ & $\begin{array}{l}97,54 \\
97,77 \\
97,85 \\
98,38\end{array}$ \\
\hline
\end{tabular}


Tabela C.4: Estimativas das Réplicas $n=1000$ pelo Modelo Paramétrico (Frank)

\begin{tabular}{|c|c|c|c|c|c|c|c|c|c|c|c|c|c|c|c|c|c|}
\hline \multicolumn{18}{|c|}{ Cenário: $n=1000, N=1300, \alpha_{t}=2, \lambda_{t}=2$ e $\alpha_{c}=\frac{1}{5}$} \\
\hline & & & \multicolumn{5}{|c|}{$\widetilde{\tau}=0,8$} & \multicolumn{5}{|c|}{$\tilde{\tau}=0,5$} & \multicolumn{5}{|c|}{$\tilde{\tau}=0$} \\
\hline & & & Viés & EPMC & EPAM & EQM & $\mathrm{CP} \%$ & Viés & EPMC & EPAM & EQM & $\mathrm{CP} \%$ & Viés & EPMC & EPAM & EQM & $\mathrm{CP} \%$ \\
\hline$\tau=0,8$ & $\pi_{c}=36,18 \%$ & $\begin{array}{l}\beta_{1} \\
\beta_{2} \\
\phi_{1} \\
\phi_{2} \\
\end{array}$ & $\begin{array}{r}-0,001 \\
0,002 \\
-0,003 \\
0,002 \\
\end{array}$ & $\begin{array}{l}0,130 \\
0,088 \\
0,138 \\
0,102 \\
\end{array}$ & $\begin{array}{l}0,147 \\
0,089 \\
0,146 \\
0,104 \\
\end{array}$ & $\begin{array}{l}0,017 \\
0,008 \\
0,019 \\
0,010 \\
\end{array}$ & $\begin{array}{l}98,38 \\
95,92 \\
96,85 \\
96,23 \\
\end{array}$ & $\begin{array}{r}-0,144 \\
0,037 \\
0,190 \\
-0,034 \\
\end{array}$ & $\begin{array}{l}0,190 \\
0,154 \\
0,206 \\
0,118 \\
\end{array}$ & $\begin{array}{l}0,201 \\
0,168 \\
0,218 \\
0,129 \\
\end{array}$ & $\begin{array}{l}0,057 \\
0,025 \\
0,079 \\
0,015 \\
\end{array}$ & $\begin{array}{l}91,77 \\
97,38 \\
89,23 \\
96,62 \\
\end{array}$ & $\begin{array}{r}-0,305 \\
-0,113 \\
-0,385 \\
0,091 \\
\end{array}$ & $\begin{array}{l}0,180 \\
0,129 \\
0,186 \\
0,156 \\
\end{array}$ & $\begin{array}{l}0,195 \\
0,141 \\
0,200 \\
0,175 \\
\end{array}$ & $\begin{array}{l}0,125 \\
0,029 \\
0,183 \\
0,033 \\
\end{array}$ & $\begin{array}{l}69,69 \\
91,00 \\
54,92 \\
95,54 \\
\end{array}$ \\
\hline$\tau=0,5$ & $\pi_{c}=38,44 \%$ & $\begin{array}{l}\beta_{1} \\
\beta_{2} \\
\phi_{1} \\
\phi_{2} \\
\end{array}$ & $\begin{array}{r}-0,095 \\
0,052 \\
0,036 \\
0,047 \\
\end{array}$ & $\begin{array}{l}0,239 \\
0,105 \\
0,250 \\
0,135 \\
\end{array}$ & $\begin{array}{l}0,255 \\
0,115 \\
0,265 \\
0,153\end{array}$ & $\begin{array}{l}0,066 \\
0,014 \\
0,064 \\
0,020\end{array}$ & $\begin{array}{l}96,54 \\
95,92 \\
97,23 \\
97,31 \\
\end{array}$ & $\begin{array}{r}-0,001 \\
-0,001 \\
0,013 \\
-0,001 \\
\end{array}$ & $\begin{array}{l}0,079 \\
0,064 \\
0,128 \\
0,092\end{array}$ & $\begin{array}{l}0,090 \\
0,081 \\
0,143 \\
0,102 \\
\end{array}$ & $\begin{array}{l}0,006 \\
0,004 \\
0,017 \\
0,008 \\
\end{array}$ & $\begin{array}{l}98,23 \\
99,23 \\
97,77 \\
97,54 \\
\end{array}$ & $\begin{array}{r}-0,217 \\
0,058 \\
-0,001 \\
0,071 \\
\end{array}$ & $\begin{array}{l}0,248 \\
0,131 \\
0,199 \\
0,143\end{array}$ & $\begin{array}{l}0,261 \\
0,142 \\
0,216 \\
0,162 \\
\end{array}$ & $\begin{array}{l}0,109 \\
0,021 \\
0,040 \\
0,025\end{array}$ & $\begin{array}{l}90,31 \\
96,62 \\
97,69 \\
97,31 \\
\end{array}$ \\
\hline$\tau=0$ & $=42,90 \%$ & $\begin{array}{l}\beta_{1} \\
\beta_{2} \\
\phi_{1} \\
\phi_{2}\end{array}$ & $\begin{array}{r}0,110 \\
0,061 \\
-0,020 \\
-0,103\end{array}$ & $\begin{array}{l}0,297 \\
0,179 \\
0,308 \\
0,213\end{array}$ & $\begin{array}{l}0,299 \\
0,187 \\
0,311 \\
0,219\end{array}$ & $\begin{array}{l}0,100 \\
0,036 \\
0,095 \\
0,056\end{array}$ & $\begin{array}{l}95,00 \\
95,92 \\
95,46 \\
94,85\end{array}$ & $\begin{array}{r}0,024 \\
-0,034 \\
-0,059 \\
-0,116\end{array}$ & $\begin{array}{l}0,216 \\
0,137 \\
0,296 \\
0,197\end{array}$ & $\begin{array}{l}0,229 \\
0,155 \\
0,307 \\
0,213\end{array}$ & $\begin{array}{l}0,047 \\
0,020 \\
0,091 \\
0,052\end{array}$ & $\begin{array}{l}97,00 \\
97,92 \\
96,38 \\
94,38\end{array}$ & $\begin{array}{r}0,000 \\
-0,001 \\
-0,005 \\
-0,003\end{array}$ & $\begin{array}{l}0,078 \\
0,069 \\
0,087 \\
0,063\end{array}$ & $\begin{array}{l}0,090 \\
0,088 \\
0,104 \\
0,081\end{array}$ & $\begin{array}{l}0,006 \\
0,005 \\
0,008 \\
0,004\end{array}$ & $\begin{array}{l}98,69 \\
99,54 \\
99,00 \\
99,46\end{array}$ \\
\hline \multicolumn{18}{|c|}{ Cenário: $n=1000, N=1300, \alpha_{t}=2, \lambda_{t}=2,5$ e $\alpha_{c}=\frac{1}{3}$} \\
\hline & & & \multicolumn{5}{|c|}{$\bar{\tau}=0,8$} & \multicolumn{5}{|c|}{$\tilde{\tau}=0,5$} & \multicolumn{5}{|c|}{$\tilde{\tau}=0$} \\
\hline & & & Viés & EPMC & EPAM & EQM & $\mathrm{CP} \%$ & Viés & EPMC & EPAM & EQM & $\mathrm{CP} \%$ & Viés & EPMC & EPAM & EQM & $\mathrm{CP} \%$ \\
\hline$\tau=0,8$ & $\pi_{c}=70,62 \%$ & $\begin{array}{l}\beta_{1} \\
\beta_{2} \\
\phi_{1} \\
\phi_{2}\end{array}$ & $\begin{array}{r}-0,011 \\
0,000 \\
-0,009 \\
-0,001\end{array}$ & $\begin{array}{l}0,153 \\
0,123 \\
0,111 \\
0,101\end{array}$ & $\begin{array}{l}0,165 \\
0,136 \\
0,129 \\
0,120\end{array}$ & $\begin{array}{l}0,024 \\
0,015 \\
0,012 \\
0,010\end{array}$ & $\begin{array}{l}97,92 \\
98,15 \\
98,38 \\
98,77\end{array}$ & $\begin{array}{r}-0,173 \\
-0,024 \\
0,174 \\
-0,025\end{array}$ & $\begin{array}{l}0,204 \\
0,126 \\
0,201 \\
0,104\end{array}$ & $\begin{array}{l}0,220 \\
0,138 \\
0,220 \\
0,122\end{array}$ & $\begin{array}{l}0,072 \\
0,016 \\
0,071 \\
0,011\end{array}$ & $\begin{array}{l}91,23 \\
97,31 \\
92,08 \\
97,77\end{array}$ & $\begin{array}{r}-0,298 \\
-0,092 \\
-0,188 \\
0,084\end{array}$ & $\begin{array}{l}0,189 \\
0,100 \\
0,172 \\
0,132\end{array}$ & $\begin{array}{l}0,208 \\
0,116 \\
0,189 \\
0,147\end{array}$ & $\begin{array}{l}0,125 \\
0,018 \\
0,065 \\
0,024\end{array}$ & $\begin{array}{l}75,00 \\
92,08 \\
86,23 \\
95,15\end{array}$ \\
\hline$\tau=0,5$ & $\pi_{c}=67,06 \%$ & $\begin{array}{l}\beta_{1} \\
\beta_{2} \\
\phi_{1} \\
\phi_{2}\end{array}$ & $\begin{array}{r}-0,206 \\
0,026 \\
0,037 \\
0,018\end{array}$ & $\begin{array}{l}0,290 \\
0,165 \\
0,231 \\
0,115\end{array}$ & $\begin{array}{l}0,302 \\
0,177 \\
0,248 \\
0,128\end{array}$ & $\begin{array}{l}0,127 \\
0,028 \\
0,055 \\
0,014\end{array}$ & $\begin{array}{l}92,62 \\
96,46 \\
96,46 \\
97,08\end{array}$ & $\begin{array}{r}0,000 \\
0,000 \\
0,001 \\
-0,001\end{array}$ & $\begin{array}{l}0,097 \\
0,078 \\
0,098 \\
0,075\end{array}$ & $\begin{array}{l}0,110 \\
0,096 \\
0,116 \\
0,092\end{array}$ & $\begin{array}{l}0,009 \\
0,006 \\
0,010 \\
0,006\end{array}$ & $\begin{array}{l}97,92 \\
99,08 \\
98,92 \\
99,08\end{array}$ & $\begin{array}{r}-0,500 \\
0,082 \\
0,080 \\
0,104\end{array}$ & $\begin{array}{l}0,276 \\
0,165 \\
0,292 \\
0,111\end{array}$ & $\begin{array}{l}0,290 \\
0,181 \\
0,303 \\
0,124\end{array}$ & $\begin{array}{l}0,326 \\
0,034 \\
0,092 \\
0,023\end{array}$ & $\begin{array}{l}62,31 \\
95,77 \\
96,00 \\
91,23\end{array}$ \\
\hline$\tau=0$ & $\pi_{c}=62,90 \%$ & $\begin{array}{l}\beta_{1} \\
\beta_{2} \\
\phi_{1} \\
\phi_{2} \\
\end{array}$ & $\begin{array}{r}0,119 \\
0,023 \\
-0,041 \\
-0,118 \\
\end{array}$ & $\begin{array}{l}0,305 \\
0,204 \\
0,316 \\
0,218 \\
\end{array}$ & $\begin{array}{l}0,310 \\
0,212 \\
0,325 \\
0,220\end{array}$ & $\begin{array}{l}0,107 \\
0,042 \\
0,102 \\
0,061\end{array}$ & $\begin{array}{l}95,31 \\
96,23 \\
96,15 \\
92,92 \\
\end{array}$ & $\begin{array}{r}0,040 \\
-0,014 \\
-0,060 \\
-0,116 \\
\end{array}$ & $\begin{array}{l}0,296 \\
0,138 \\
0,297 \\
0,198\end{array}$ & $\begin{array}{l}0,311 \\
0,149 \\
0,314 \\
0,212 \\
\end{array}$ & $\begin{array}{l}0,089 \\
0,019 \\
0,092 \\
0,053 \\
\end{array}$ & $\begin{array}{l}97,77 \\
97,92 \\
97,31 \\
94,62 \\
\end{array}$ & $\begin{array}{r}-0,001 \\
0,001 \\
0,001 \\
0,001 \\
\end{array}$ & $\begin{array}{l}0,089 \\
0,080 \\
0,090 \\
0,084\end{array}$ & $\begin{array}{l}0,102 \\
0,091 \\
0,107 \\
0,103 \\
\end{array}$ & $\begin{array}{l}0,008 \\
0,006 \\
0,008 \\
0,007 \\
\end{array}$ & $\begin{array}{l}98,00 \\
97,92 \\
98,38 \\
98,69 \\
\end{array}$ \\
\hline
\end{tabular}

Tabela C.5: Estimativas das Réplicas $n=300$ pelo Modelo Piecewise (Clayton)

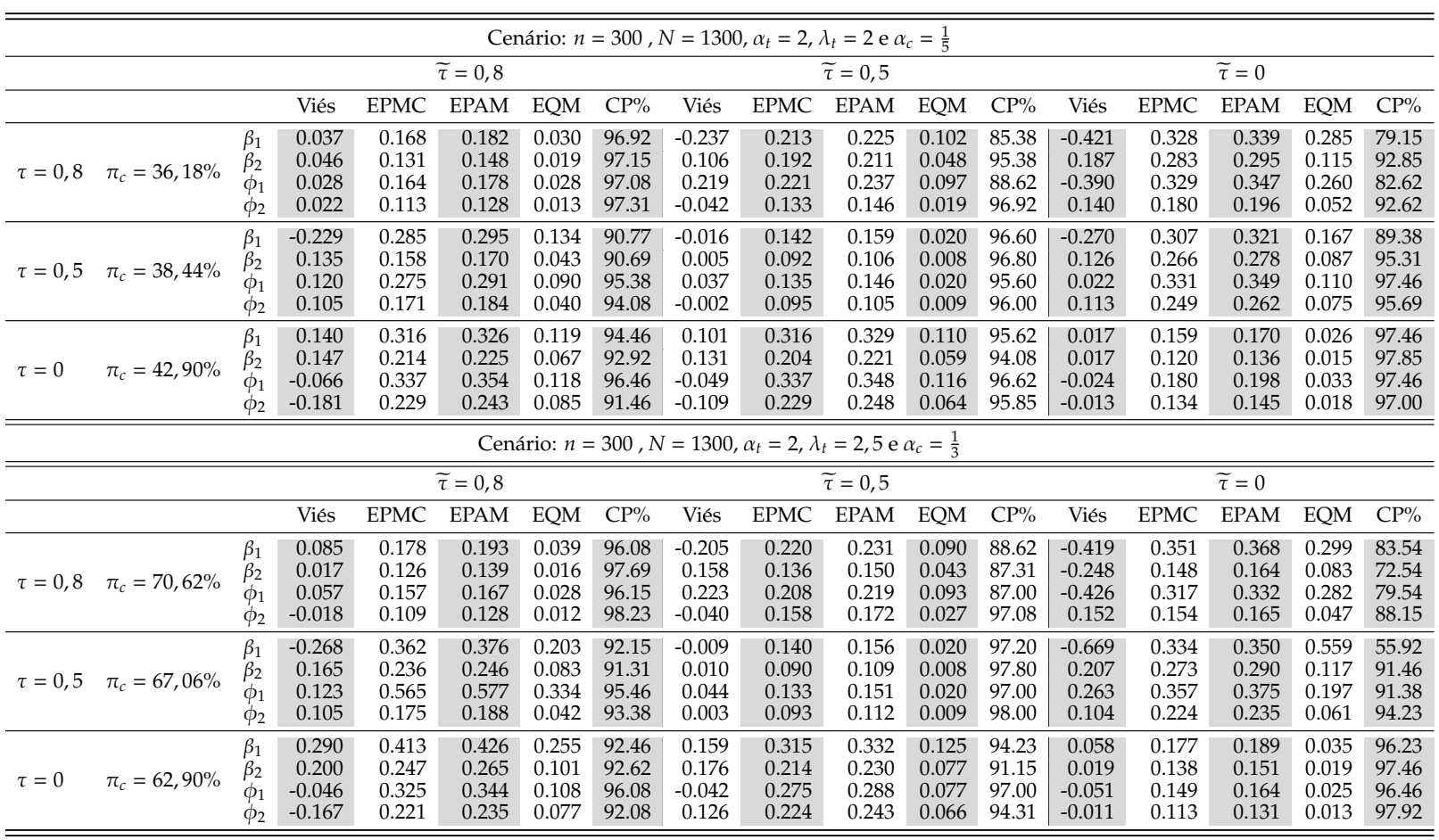


Tabela C.6: Estimativas das Réplicas $n=1000$ pelo Modelo Piecewise (Clayton)

\begin{tabular}{|c|c|c|c|c|c|c|c|c|c|c|c|c|c|c|c|c|c|}
\hline \multicolumn{18}{|c|}{ Cenário: $n=1000, N=1300, \alpha_{t}=2, \lambda_{t}=2$ e $\alpha_{c}=\frac{1}{5}$} \\
\hline & & & \multicolumn{5}{|c|}{$\widetilde{\tau}=0,8$} & \multicolumn{5}{|c|}{$\widetilde{\tau}=0,5$} & \multicolumn{5}{|c|}{$\tilde{\tau}=0$} \\
\hline & & & Viés & EPMC & EPAM & EQM & $\mathrm{CP} \%$ & Viés & EPMC & EPAM & EQM & $\mathrm{CP} \%$ & Viés & EPMC & EPAM & EQM & $\mathrm{CP} \%$ \\
\hline$\tau=0,8$ & $\pi_{c}=36,18 \%$ & $\begin{array}{l}\beta_{1} \\
\beta_{2} \\
\phi_{1} \\
\phi_{2} \\
\end{array}$ & $\begin{array}{r}0,007 \\
-0,006 \\
0,015 \\
-0,008 \\
\end{array}$ & $\begin{array}{l}0,158 \\
0,119 \\
0,167 \\
0,121 \\
\end{array}$ & $\begin{array}{l}0,171 \\
0,134 \\
0,177 \\
0,137 \\
\end{array}$ & $\begin{array}{l}0,025 \\
0,014 \\
0,028 \\
0,015\end{array}$ & $\begin{array}{l}96,85 \\
97,38 \\
96,46 \\
97,46 \\
\end{array}$ & $\begin{array}{r}-0,200 \\
0,053 \\
0,227 \\
-0,037\end{array}$ & $\begin{array}{l}0,196 \\
0,181 \\
0,205 \\
0,120\end{array}$ & $\begin{array}{l}0,215 \\
0,196 \\
0,223 \\
0,132\end{array}$ & $\begin{array}{l}0,078 \\
0,036 \\
0,094 \\
0,016\end{array}$ & $\begin{array}{l}90,31 \\
96,23 \\
86,62 \\
97,31\end{array}$ & $\begin{array}{r}-0,337 \\
-0,118 \\
-0,406 \\
0,132\end{array}$ & $\begin{array}{l}0,277 \\
0,163 \\
0,318 \\
0,190\end{array}$ & $\begin{array}{l}0,288 \\
0,182 \\
0,332 \\
0,204\end{array}$ & $\begin{array}{l}0,190 \\
0,040 \\
0,266 \\
0,054\end{array}$ & $\begin{array}{l}81,69 \\
93,85 \\
80,54 \\
93,00\end{array}$ \\
\hline$\tau=0,5$ & $\pi_{c}=38,44 \%$ & $\begin{array}{l}\beta_{1} \\
\beta_{2} \\
\phi_{1} \\
\phi_{2} \\
\end{array}$ & $\begin{array}{r}-0,178 \\
0,102 \\
0,112 \\
0,107 \\
\end{array}$ & $\begin{array}{l}0,271 \\
0,144 \\
0,250 \\
0,152 \\
\end{array}$ & $\begin{array}{l}0,286 \\
0,162 \\
0,266 \\
0,166 \\
\end{array}$ & $\begin{array}{l}0,105 \\
0,031 \\
0,075 \\
0,035 \\
\end{array}$ & $\begin{array}{l}93,23 \\
93,62 \\
94,85 \\
92,92 \\
\end{array}$ & $\begin{array}{r}-0,007 \\
0,006 \\
-0,018 \\
0,007\end{array}$ & $\begin{array}{l}0,112 \\
0,081 \\
0,095 \\
0,087\end{array}$ & $\begin{array}{l}0,116 \\
0,085 \\
0,099 \\
0,095\end{array}$ & $\begin{array}{l}0,013 \\
0,007 \\
0,009 \\
0,008 \\
\end{array}$ & $\begin{array}{l}95,60 \\
95,40 \\
95,40 \\
96,40 \\
\end{array}$ & $\begin{array}{r}-0,242 \\
0,084 \\
0,020 \\
0,111 \\
\end{array}$ & $\begin{array}{l}0,308 \\
0,241 \\
0,313 \\
0,221\end{array}$ & $\begin{array}{l}0,323 \\
0,252 \\
0,323 \\
0,231 \\
\end{array}$ & $\begin{array}{l}0,153 \\
0,065 \\
0,098 \\
0,061 \\
\end{array}$ & $\begin{array}{l}91,46 \\
95,23 \\
96,69 \\
93,77 \\
\end{array}$ \\
\hline$\tau=0$ & $\pi_{c}=42,90 \%$ & $\begin{array}{l}\beta_{1} \\
\beta_{2} \\
\phi_{1} \\
\phi_{2}\end{array}$ & $\begin{array}{r}0,109 \\
0,075 \\
-0,046 \\
-0,167\end{array}$ & $\begin{array}{l}0,324 \\
0,200 \\
0,312 \\
0,206\end{array}$ & $\begin{array}{l}0,336 \\
0,210 \\
0,330 \\
0,222\end{array}$ & $\begin{array}{l}0,117 \\
0,046 \\
0,099 \\
0,070\end{array}$ & $\begin{array}{l}96,00 \\
95,85 \\
96,69 \\
91,54\end{array}$ & $\begin{array}{r}0,079 \\
0,064 \\
-0,043 \\
-0,105\end{array}$ & $\begin{array}{l}0,249 \\
0,198 \\
0,311 \\
0,220\end{array}$ & $\begin{array}{l}0,259 \\
0,212 \\
0,329 \\
0,234\end{array}$ & $\begin{array}{l}0,068 \\
0,043 \\
0,099 \\
0,059\end{array}$ & $\begin{array}{l}95,46 \\
96,00 \\
96,62 \\
95,08\end{array}$ & $\begin{array}{r}0,022 \\
0,011 \\
-0,018 \\
-0,008\end{array}$ & $\begin{array}{l}0,129 \\
0,100 \\
0,150 \\
0,114\end{array}$ & $\begin{array}{l}0,143 \\
0,116 \\
0,162 \\
0,130\end{array}$ & $\begin{array}{l}0,017 \\
0,010 \\
0,023 \\
0,013\end{array}$ & $\begin{array}{l}97,69 \\
98,38 \\
97,00 \\
98,08\end{array}$ \\
\hline \multicolumn{18}{|c|}{ Cenário: $n=1000, N=1300, \alpha_{t}=2, \lambda_{t}=2,5$ e $\alpha_{c}=\frac{1}{3}$} \\
\hline & & & \multicolumn{5}{|c|}{$\widetilde{\tau}=0,8$} & \multicolumn{5}{|c|}{$\tilde{\tau}=0,5$} & \multicolumn{5}{|c|}{$\tilde{\tau}=0$} \\
\hline & & & Viés & EPMC & EPAM & EQM & $\mathrm{CP} \%$ & Viés & EPMC & EPAM & EQM & $\mathrm{CP} \%$ & Viés & EPMC & EPAM & EQM & $\mathrm{CP} \%$ \\
\hline$\tau=0,8$ & $\pi_{\mathcal{C}}=70,62 \%$ & $\begin{array}{l}\beta_{1} \\
\beta_{2} \\
\phi_{1} \\
\phi_{2}\end{array}$ & $\begin{array}{r}0,017 \\
0,006 \\
0,020 \\
-0,005\end{array}$ & $\begin{array}{l}0,167 \\
0,120 \\
0,125 \\
0,097\end{array}$ & $\begin{array}{l}0,179 \\
0,139 \\
0,139 \\
0,113\end{array}$ & $\begin{array}{l}0,028 \\
0,014 \\
0,016 \\
0,009\end{array}$ & $\begin{array}{l}97,38 \\
98,62 \\
98,00 \\
98,77\end{array}$ & $\begin{array}{r}-0,206 \\
0,079 \\
0,221 \\
-0,040\end{array}$ & $\begin{array}{l}0,205 \\
0,127 \\
0,190 \\
0,105\end{array}$ & $\begin{array}{l}0,224 \\
0,142 \\
0,205 \\
0,119\end{array}$ & $\begin{array}{l}0,084 \\
0,022 \\
0,085 \\
0,013\end{array}$ & $\begin{array}{l}89,08 \\
95,38 \\
85,46 \\
97,08\end{array}$ & $\begin{array}{r}-0,343 \\
-0,201 \\
-0,412 \\
0,128\end{array}$ & $\begin{array}{l}0,277 \\
0,114 \\
0,317 \\
0,190\end{array}$ & $\begin{array}{l}0,288 \\
0,126 \\
0,328 \\
0,206\end{array}$ & $\begin{array}{l}0,194 \\
0,053 \\
0,270 \\
0,052\end{array}$ & $\begin{array}{l}81,46 \\
68,54 \\
79,46 \\
93,92 \\
\end{array}$ \\
\hline$\tau=0,5$ & $\pi_{c}=67,06 \%$ & $\begin{array}{l}\beta_{1} \\
\beta_{2} \\
\beta_{2} \\
\beta_{2}\end{array}$ & $\begin{array}{r}-0,232 \\
0,151 \\
0,117 \\
0,110\end{array}$ & $\begin{array}{l}0,297 \\
0,182 \\
0,247 \\
0,150\end{array}$ & $\begin{array}{l}0,308 \\
0,201 \\
0,257 \\
0,168\end{array}$ & $\begin{array}{l}0,142 \\
0,056 \\
0,075 \\
0,035\end{array}$ & $\begin{array}{l}91,54 \\
91,85 \\
94,08 \\
93,54\end{array}$ & $\begin{array}{r}-0,012 \\
0,002 \\
-0,022 \\
0,003\end{array}$ & $\begin{array}{l}0,103 \\
0,075 \\
0,088 \\
0,081\end{array}$ & $\begin{array}{l}0,105 \\
0,083 \\
0,097 \\
0,089\end{array}$ & $\begin{array}{l}0,011 \\
0,006 \\
0,008 \\
0,007\end{array}$ & $\begin{array}{l}94,00 \\
96,20 \\
95,60 \\
95,80\end{array}$ & $\begin{array}{r}-0,624 \\
0,183 \\
0,017 \\
0,110\end{array}$ & $\begin{array}{l}0,252 \\
0,204 \\
0,298 \\
0,194\end{array}$ & $\begin{array}{l}0,270 \\
0,222 \\
0,316 \\
0,208\end{array}$ & $\begin{array}{l}0,453 \\
0,075 \\
0,089 \\
0,050\end{array}$ & $\begin{array}{l}38,00 \\
90,23 \\
97,00 \\
94,62\end{array}$ \\
\hline$\tau=0$ & $\pi_{c}=62,90 \%$ & $\begin{array}{l}\beta_{1} \\
\beta_{2} \\
\phi_{1} \\
\phi_{2}\end{array}$ & $\begin{array}{r}0,179 \\
0,100 \\
-0,053 \\
-0,172\end{array}$ & $\begin{array}{l}0,313 \\
0,207 \\
0,322 \\
0,213\end{array}$ & $\begin{array}{l}0,331 \\
0,225 \\
0,336 \\
0,223\end{array}$ & $\begin{array}{l}0,130 \\
0,053 \\
0,106 \\
0,075\end{array}$ & $\begin{array}{l}94,85 \\
96,08 \\
96,31 \\
91,15\end{array}$ & $\begin{array}{r}0,115 \\
-0,030 \\
-0,050 \\
-0,110\end{array}$ & $\begin{array}{l}0,308 \\
0,207 \\
0,307 \\
0,217\end{array}$ & $\begin{array}{l}0,327 \\
0,221 \\
0,320 \\
0,230\end{array}$ & $\begin{array}{l}0,108 \\
0,044 \\
0,097 \\
0,059\end{array}$ & $\begin{array}{l}96,23 \\
97,00 \\
96,62 \\
94,69\end{array}$ & $\begin{array}{r}0,021 \\
0,021 \\
-0,019 \\
-0,009\end{array}$ & $\begin{array}{l}0,132 \\
0,118 \\
0,154 \\
0,117\end{array}$ & $\begin{array}{l}0,145 \\
0,134 \\
0,166 \\
0,130\end{array}$ & $\begin{array}{l}0,018 \\
0,014 \\
0,024 \\
0,014\end{array}$ & $\begin{array}{l}96,77 \\
97,85 \\
97,08 \\
97,69\end{array}$ \\
\hline
\end{tabular}

Tabela C.7: Estimativas das Réplicas $n=300$ pelo Modelo Piecewise (Frank)

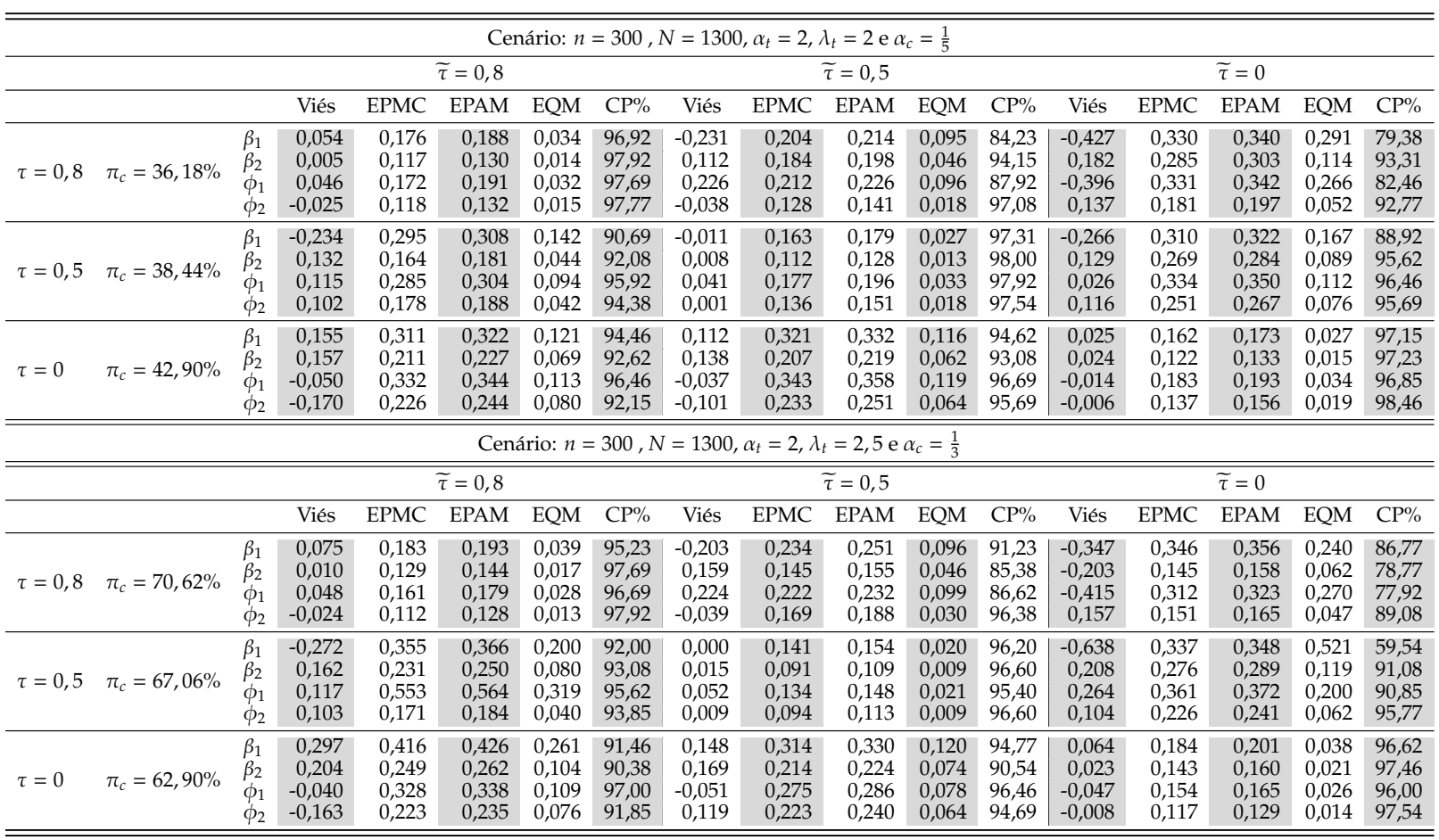


Tabela C.8: Estimativas das Réplicas $n=1000$ pelo Modelo Piecewise (Frank)

\begin{tabular}{|c|c|c|c|c|c|c|c|c|c|c|c|c|c|c|c|c|c|}
\hline \multicolumn{18}{|c|}{ Cenário: $n=1000, N=1300, \alpha_{t}=2, \lambda_{t}=2$ e $\alpha_{c}=\frac{1}{5}$} \\
\hline & & & \multicolumn{5}{|c|}{$\widetilde{\tau}=0,8$} & \multicolumn{5}{|c|}{$\widetilde{\tau}=0,5$} & \multicolumn{5}{|c|}{$\tilde{\tau}=0$} \\
\hline & & & Viés & EPMC & EPAM & EQM & $\mathrm{CP} \%$ & Viés & EPMC & EPAM & EQM & $\mathrm{CP} \%$ & Viés & EPMC & EPAM & EQM & $\mathrm{CP} \%$ \\
\hline$\tau=0,8$ & $\pi_{c}=36,18 \%$ & $\begin{array}{l}\beta_{1} \\
\beta_{2} \\
\phi_{1} \\
\phi_{2} \\
\end{array}$ & $\begin{array}{r}0,006 \\
-0,007 \\
0,015 \\
-0,009 \\
\end{array}$ & $\begin{array}{l}0,150 \\
0,113 \\
0,159 \\
0,115 \\
\end{array}$ & $\begin{array}{l}0,162 \\
0,128 \\
0,169 \\
0,132 \\
\end{array}$ & $\begin{array}{l}0,023 \\
0,013 \\
0,026 \\
0,013 \\
\end{array}$ & $\begin{array}{l}97,08 \\
97,85 \\
96,85 \\
98,00 \\
\end{array}$ & $\begin{array}{r}-0,193 \\
0,060 \\
0,235 \\
-0,033 \\
\end{array}$ & $\begin{array}{l}0,191 \\
0,176 \\
0,200 \\
0,117 \\
\end{array}$ & $\begin{array}{l}0,208 \\
0,189 \\
0,216 \\
0,132 \\
\end{array}$ & $\begin{array}{l}0,074 \\
0,035 \\
0,095 \\
0,015 \\
\end{array}$ & $\begin{array}{l}89,00 \\
96,46 \\
85,31 \\
97,38 \\
\end{array}$ & $\begin{array}{r}-0,343 \\
-0,122 \\
-0,412 \\
0,128 \\
\end{array}$ & $\begin{array}{l}0,282 \\
0,166 \\
0,323 \\
0,193 \\
\end{array}$ & $\begin{array}{l}0,298 \\
0,176 \\
0,340 \\
0,209 \\
\end{array}$ & $\begin{array}{l}0,197 \\
0,042 \\
0,274 \\
0,054 \\
\end{array}$ & $\begin{array}{l}82,69 \\
93,08 \\
80,54 \\
93,54 \\
\end{array}$ \\
\hline$\tau=0,5$ & $\pi_{c}=38,44 \%$ & $\begin{array}{l}\beta_{1} \\
\beta_{2} \\
\phi_{1} \\
\phi_{2} \\
\end{array}$ & $\begin{array}{r}-0,167 \\
0,107 \\
0,122 \\
0,113 \\
\end{array}$ & $\begin{array}{l}0,278 \\
0,148 \\
0,257 \\
0,157 \\
\end{array}$ & $\begin{array}{l}0,295 \\
0,164 \\
0,269 \\
0,172\end{array}$ & $\begin{array}{l}0,105 \\
0,033 \\
0,081 \\
0,037\end{array}$ & $\begin{array}{l}94,46 \\
93,23 \\
94,31 \\
93,08 \\
\end{array}$ & $\begin{array}{r}-0,009 \\
0,005 \\
-0,020 \\
0,006\end{array}$ & $\begin{array}{l}0,109 \\
0,079 \\
0,093 \\
0,085\end{array}$ & $\begin{array}{l}0,116 \\
0,088 \\
0,097 \\
0,094\end{array}$ & $\begin{array}{l}0,012 \\
0,006 \\
0,009 \\
0,007 \\
\end{array}$ & $\begin{array}{l}97,20 \\
97,20 \\
96,40 \\
97,20\end{array}$ & $\begin{array}{r}-0,235 \\
0,090 \\
0,027 \\
0,116 \\
\end{array}$ & $\begin{array}{l}0,306 \\
0,240 \\
0,311 \\
0,220\end{array}$ & $\begin{array}{l}0,324 \\
0,251 \\
0,326 \\
0,235 \\
\end{array}$ & $\begin{array}{l}0,149 \\
0,066 \\
0,097 \\
0,062 \\
\end{array}$ & $\begin{array}{l}91,85 \\
95,85 \\
96,46 \\
94,92 \\
\end{array}$ \\
\hline$\tau=0$ & $=42,90 \%$ & $\begin{array}{l}\beta_{1} \\
\beta_{2} \\
\phi_{1} \\
\phi_{2} \\
\end{array}$ & $\begin{array}{r}0,113 \\
0,077 \\
-0,043 \\
-0,165 \\
\end{array}$ & $\begin{array}{l}0,309 \\
0,191 \\
0,298 \\
0,197 \\
\end{array}$ & $\begin{array}{l}0,320 \\
0,201 \\
0,314 \\
0,215 \\
\end{array}$ & $\begin{array}{l}0,108 \\
0,042 \\
0,091 \\
0,066\end{array}$ & $\begin{array}{l}95,15 \\
95,31 \\
96,69 \\
92,31 \\
\end{array}$ & $\begin{array}{r}0,075 \\
0,061 \\
-0,049 \\
-0,109 \\
\end{array}$ & $\begin{array}{l}0,239 \\
0,190 \\
0,298 \\
0,211 \\
\end{array}$ & $\begin{array}{l}0,249 \\
0,206 \\
0,314 \\
0,223 \\
\end{array}$ & $\begin{array}{l}0,063 \\
0,040 \\
0,091 \\
0,056 \\
\end{array}$ & $\begin{array}{l}96,54 \\
97,00 \\
96,62 \\
94,46 \\
\end{array}$ & $\begin{array}{r}0,019 \\
0,009 \\
-0,021 \\
-0,011 \\
\end{array}$ & $\begin{array}{l}0,126 \\
0,098 \\
0,146 \\
0,112 \\
\end{array}$ & $\begin{array}{l}0,137 \\
0,113 \\
0,157 \\
0,128 \\
\end{array}$ & $\begin{array}{l}0,016 \\
0,010 \\
0,022 \\
0,013 \\
\end{array}$ & $\begin{array}{l}96,85 \\
98,00 \\
97,08 \\
98,15 \\
\end{array}$ \\
\hline \multicolumn{18}{|c|}{ Cenário: $n=1000, N=1300, \alpha_{t}=2, \lambda_{t}=2,5$ e $\alpha_{c}=\frac{1}{3}$} \\
\hline & & & \multicolumn{5}{|c|}{$\tilde{\tau}=0,8$} & \multicolumn{5}{|c|}{$\tilde{\bar{\tau}}=0,5$} & \multicolumn{5}{|c|}{$\tilde{\tau}=0$} \\
\hline & & & Viés & EPMC & EPAM & EQM & $\mathrm{CP} \%$ & Viés & EPMC & EPAM & EQM & $\mathrm{CP} \%$ & Viés & EPMC & EPAM & EQM & $\mathrm{CP} \%$ \\
\hline$\tau=0,8$ & $\pi_{c}=70,62 \%$ & $\begin{array}{l}\beta_{1} \\
\beta_{2} \\
\phi_{1} \\
\phi_{2}\end{array}$ & $\begin{array}{r}0,014 \\
0,004 \\
0,018 \\
-0,006\end{array}$ & $\begin{array}{l}0,174 \\
0,125 \\
0,130 \\
0,101\end{array}$ & $\begin{array}{l}0,186 \\
0,138 \\
0,142 \\
0,117\end{array}$ & $\begin{array}{l}0,030 \\
0,016 \\
0,017 \\
0,010\end{array}$ & $\begin{array}{l}97,23 \\
97,46 \\
97,23 \\
98,23\end{array}$ & $\begin{array}{r}-0,202 \\
0,082 \\
0,225 \\
-0,038\end{array}$ & $\begin{array}{l}0,215 \\
0,133 \\
0,200 \\
0,111\end{array}$ & $\begin{array}{l}0,225 \\
0,150 \\
0,215 \\
0,127\end{array}$ & $\begin{array}{l}0,087 \\
0,024 \\
0,091 \\
0,014\end{array}$ & $\begin{array}{l}87,77 \\
95,92 \\
86,69 \\
97,46\end{array}$ & $\begin{array}{r}-0,335 \\
-0,198 \\
-0,404 \\
0,133\end{array}$ & $\begin{array}{l}0,275 \\
0,113 \\
0,316 \\
0,189\end{array}$ & $\begin{array}{l}0,286 \\
0,126 \\
0,334 \\
0,203\end{array}$ & $\begin{array}{l}0,188 \\
0,052 \\
0,263 \\
0,053\end{array}$ & $\begin{array}{l}82,46 \\
70,62 \\
81,85 \\
92,85\end{array}$ \\
\hline$\tau=0,5$ & $\pi_{c}=67,06 \%$ & $\begin{array}{l}\beta_{1} \\
\beta_{2} \\
\phi_{1} \\
\phi_{2}\end{array}$ & $\begin{array}{r}-0,235 \\
0,149 \\
0,115 \\
0,109 \\
\end{array}$ & $\begin{array}{l}0,282 \\
0,173 \\
0,235 \\
0,143\end{array}$ & $\begin{array}{l}0,296 \\
0,183 \\
0,252 \\
0,156\end{array}$ & $\begin{array}{l}0,135 \\
0,052 \\
0,068 \\
0,032\end{array}$ & $\begin{array}{l}90,62 \\
90,15 \\
95,31 \\
92,31 \\
\end{array}$ & $\begin{array}{r}-0,009 \\
0,004 \\
-0,020 \\
0,005\end{array}$ & $\begin{array}{l}0,109 \\
0,079 \\
0,093 \\
0,085\end{array}$ & $\begin{array}{l}0,116 \\
0,083 \\
0,094 \\
0,087\end{array}$ & $\begin{array}{l}0,012 \\
0,006 \\
0,009 \\
0,007\end{array}$ & $\begin{array}{l}95,20 \\
95,20 \\
93,80 \\
94,80\end{array}$ & $\begin{array}{r}-0,616 \\
0,189 \\
0,027 \\
0,116\end{array}$ & $\begin{array}{l}0,249 \\
0,202 \\
0,294 \\
0,192\end{array}$ & $\begin{array}{l}0,262 \\
0,220 \\
0,305 \\
0,206\end{array}$ & $\begin{array}{l}0,441 \\
0,077 \\
0,087 \\
0,050\end{array}$ & $\begin{array}{l}37,23 \\
89,31 \\
97,23 \\
93,46 \\
\end{array}$ \\
\hline$\tau=0$ & $\pi_{c}=62,90 \%$ & $\begin{array}{l}\beta_{1} \\
\beta_{2} \\
\phi_{1} \\
\phi_{2} \\
\end{array}$ & $\begin{array}{r}0,166 \\
0,103 \\
-0,049 \\
-0,169 \\
\end{array}$ & $\begin{array}{l}0,301 \\
0,199 \\
0,310 \\
0,205 \\
\end{array}$ & $\begin{array}{l}0,319 \\
0,215 \\
0,325 \\
0,216\end{array}$ & $\begin{array}{l}0,118 \\
0,050 \\
0,099 \\
0,071 \\
\end{array}$ & $\begin{array}{l}94,00 \\
94,54 \\
96,08 \\
90,85 \\
\end{array}$ & $\begin{array}{r}0,095 \\
-0,033 \\
-0,055 \\
-0,113 \\
\end{array}$ & $\begin{array}{l}0,299 \\
0,201 \\
0,298 \\
0,211 \\
\end{array}$ & $\begin{array}{l}0,318 \\
0,211 \\
0,317 \\
0,221 \\
\end{array}$ & $\begin{array}{l}0,098 \\
0,041 \\
0,092 \\
0,057 \\
\end{array}$ & $\begin{array}{l}97,00 \\
97,00 \\
97,38 \\
94,69 \\
\end{array}$ & $\begin{array}{r}0,019 \\
0,019 \\
-0,021 \\
-0,011 \\
\end{array}$ & $\begin{array}{l}0,128 \\
0,114 \\
0,149 \\
0,113\end{array}$ & $\begin{array}{l}0,147 \\
0,127 \\
0,164 \\
0,132 \\
\end{array}$ & $\begin{array}{l}0,017 \\
0,013 \\
0,023 \\
0,013 \\
\end{array}$ & $\begin{array}{l}97,85 \\
97,54 \\
97,85 \\
98,62 \\
\end{array}$ \\
\hline
\end{tabular}

Tabela C.9: Estimativas das Réplicas $n=300$ pelo Modelo M-splines (Clayton)

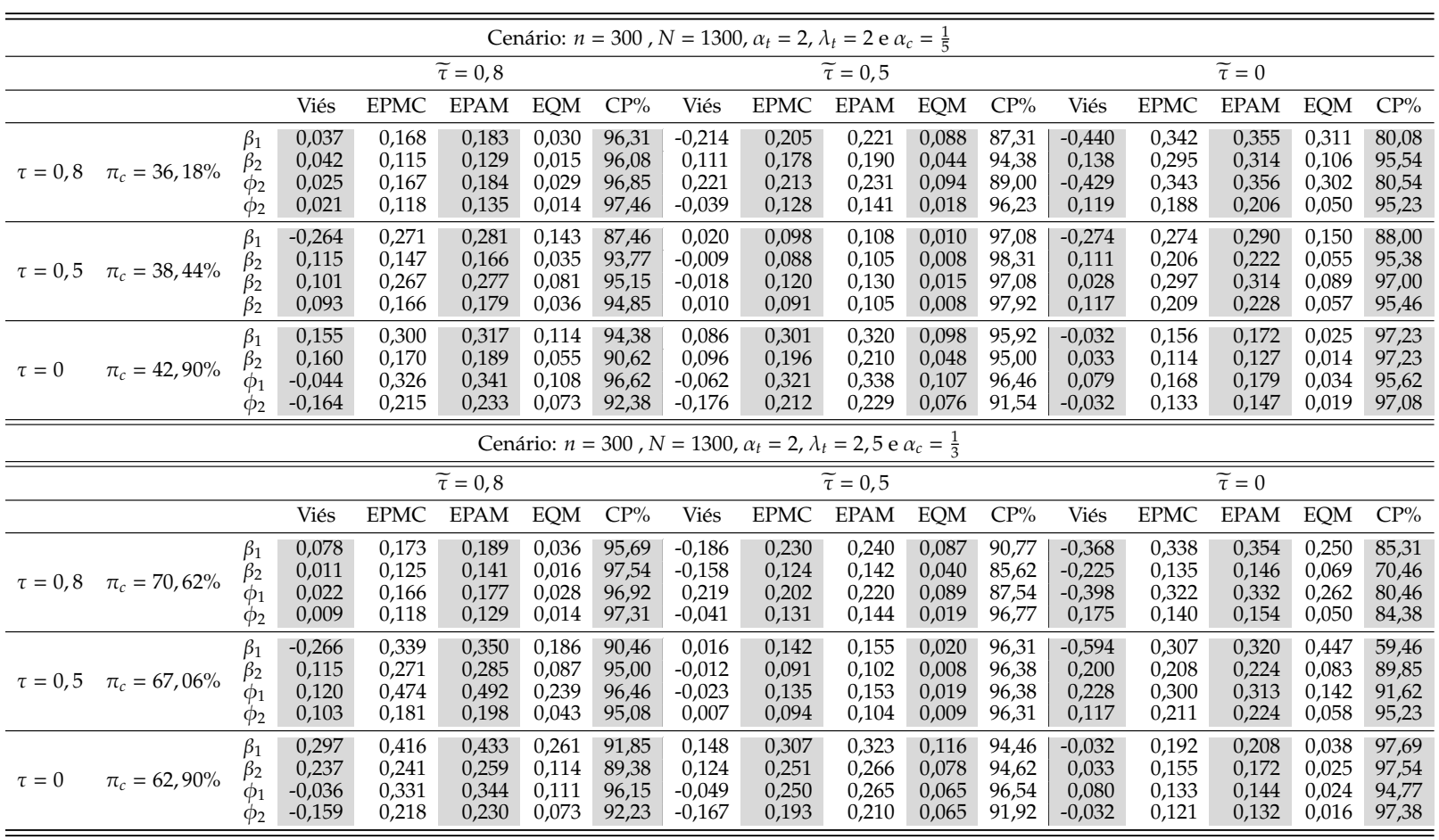


Tabela C.10: Estimativas das Réplicas $n=1000$ pelo Modelo M-splines (Clayton)

\begin{tabular}{|c|c|c|c|c|c|c|c|c|c|c|c|c|c|c|c|c|c|}
\hline \multicolumn{18}{|c|}{ Cenário: $n=1000, N=1300, \alpha_{t}=2, \lambda_{t}=2$ e $\alpha_{c}=\frac{1}{5}$} \\
\hline & & & \multicolumn{5}{|c|}{$\tilde{\tau}=0,8$} & \multicolumn{5}{|c|}{$\tilde{\tau}=0,5$} & \multicolumn{5}{|c|}{$\tilde{\tau}=0$} \\
\hline & & & Viés & EPMC & EPAM & EQM & $\mathrm{CP} \%$ & Viés & EPMC & EPAM & EQM & $\mathrm{CP} \%$ & Viés & EPMC & EPAM & EQM & $\mathrm{CP} \%$ \\
\hline$\tau=0,8$ & $\pi_{c}=36,18 \%$ & $\begin{array}{l}\beta_{1} \\
\beta_{2} \\
\phi_{1} \\
\phi_{2} \\
\end{array}$ & $\begin{array}{r}0,007 \\
0,009 \\
0,006 \\
-0,006 \\
\end{array}$ & $\begin{array}{l}0,149 \\
0,110 \\
0,144 \\
0,110 \\
\end{array}$ & $\begin{array}{l}0,167 \\
0,127 \\
0,161 \\
0,126 \\
\end{array}$ & $\begin{array}{l}0,022 \\
0,012 \\
0,021 \\
0,012\end{array}$ & $\begin{array}{l}97,85 \\
98,54 \\
97,85 \\
98,31 \\
\end{array}$ & $\begin{array}{r}-0,179 \\
0,047 \\
0,216 \\
-0,042 \\
\end{array}$ & $\begin{array}{l}0,187 \\
0,164 \\
0,204 \\
0,112 \\
\end{array}$ & $\begin{array}{l}0,203 \\
0,182 \\
0,222 \\
0,123 \\
\end{array}$ & $\begin{array}{l}0,067 \\
0,029 \\
0,088 \\
0,014 \\
\end{array}$ & $\begin{array}{l}89,92 \\
97,46 \\
87,31 \\
96,00 \\
\end{array}$ & $\begin{array}{r}-0,335 \\
-0,112 \\
-0,403 \\
0,133 \\
\end{array}$ & $\begin{array}{l}0,261 \\
0,158 \\
0,302 \\
0,176 \\
\end{array}$ & $\begin{array}{l}0,279 \\
0,174 \\
0,317 \\
0,192 \\
\end{array}$ & $\begin{array}{l}0,180 \\
0,038 \\
0,254 \\
0,049\end{array}$ & $\begin{array}{l}81,85 \\
93,62 \\
79,54 \\
93,15 \\
\end{array}$ \\
\hline$\tau=0,5$ & $8,44 \%$ & $\begin{array}{l}\beta_{1} \\
\beta_{2} \\
\phi_{1} \\
\phi_{2}\end{array}$ & $\begin{array}{r}-0,157 \\
0,069 \\
0,114 \\
0,100 \\
\end{array}$ & $\begin{array}{l}0,259 \\
0,138 \\
0,257 \\
0,169\end{array}$ & $\begin{array}{l}0,276 \\
0,152 \\
0,268 \\
0,185\end{array}$ & $\begin{array}{l}0,092 \\
0,024 \\
0,079 \\
0,039\end{array}$ & $\begin{array}{l}94,08 \\
95,54 \\
94,77 \\
94,69 \\
\end{array}$ & $\begin{array}{r}-0,009 \\
0,004 \\
-0,020 \\
0,005 \\
\end{array}$ & $\begin{array}{l}0,058 \\
0,048 \\
0,062 \\
0,055\end{array}$ & $\begin{array}{l}0,065 \\
0,055 \\
0,067 \\
0,056\end{array}$ & $\begin{array}{l}0,003 \\
0,002 \\
0,004 \\
0,003 \\
\end{array}$ & $\begin{array}{l}96,77 \\
96,85 \\
95,77 \\
94,62 \\
\end{array}$ & $\begin{array}{r}-0,213 \\
0,083 \\
0,030 \\
0,119 \\
\end{array}$ & $\begin{array}{l}0,281 \\
0,216 \\
0,307 \\
0,211\end{array}$ & $\begin{array}{l}0,300 \\
0,232 \\
0,318 \\
0,229\end{array}$ & $\begin{array}{l}0,124 \\
0,054 \\
0,095 \\
0,059\end{array}$ & $\begin{array}{l}92,54 \\
96,54 \\
97,23 \\
94,54 \\
\end{array}$ \\
\hline$\tau=0$ & $2,90 \%$ & $\begin{array}{l}\beta_{1} \\
\beta_{2} \\
\phi_{1} \\
\phi_{2}\end{array}$ & $\begin{array}{r}0,091 \\
0,108 \\
-0,088 \\
-0,167\end{array}$ & $\begin{array}{l}0,309 \\
0,190 \\
0,305 \\
0,205\end{array}$ & $\begin{array}{l}0,325 \\
0,208 \\
0,317 \\
0,221\end{array}$ & $\begin{array}{l}0,104 \\
0,048 \\
0,101 \\
0,070\end{array}$ & $\begin{array}{l}97,00 \\
95,23 \\
95,62 \\
91,92\end{array}$ & $\begin{array}{r}0,052 \\
0,070 \\
-0,056 \\
-0,172\end{array}$ & $\begin{array}{l}0,232 \\
0,157 \\
0,321 \\
0,213\end{array}$ & $\begin{array}{l}0,246 \\
0,176 \\
0,338 \\
0,223\end{array}$ & $\begin{array}{l}0,057 \\
0,030 \\
0,106 \\
0,075\end{array}$ & $\begin{array}{l}96,62 \\
96,62 \\
96,00 \\
91,23\end{array}$ & $\begin{array}{r}-0,004 \\
0,003 \\
-0,008 \\
0,004\end{array}$ & $\begin{array}{l}0,127 \\
0,091 \\
0,102 \\
0,117\end{array}$ & $\begin{array}{l}0,141 \\
0,109 \\
0,112 \\
0,129\end{array}$ & $\begin{array}{l}0,016 \\
0,008 \\
0,010 \\
0,014\end{array}$ & $\begin{array}{l}97,54 \\
98,46 \\
97,46 \\
97,54\end{array}$ \\
\hline \multicolumn{18}{|c|}{ Cenário: $n=1000, N=1300, \alpha_{t}=2, \lambda_{t}=2,5$ e $\alpha_{c}=\frac{1}{3}$} \\
\hline & & & \multicolumn{5}{|c|}{$\bar{\tau}=0,8$} & \multicolumn{5}{|c|}{$\bar{\tau}=0,5$} & \multicolumn{5}{|c|}{$\tilde{\tau}=0$} \\
\hline & & & Viés & EPMC & EPAM & EQM & $\mathrm{CP} \%$ & Viés & EPMC & EPAM & EQM & $\mathrm{CP} \%$ & Viés & EPMC & EPAM & EQM & $\mathrm{CP} \%$ \\
\hline$\tau=0,8$ & $\pi_{c}=70,62 \%$ & $\begin{array}{l}\beta_{1} \\
\beta_{2} \\
\phi_{1} \\
\phi_{2}\end{array}$ & $\begin{array}{r}0,011 \\
0,008 \\
0,010 \\
-0,006\end{array}$ & $\begin{array}{l}0,168 \\
0,123 \\
0,123 \\
0,103\end{array}$ & $\begin{array}{l}0,180 \\
0,139 \\
0,136 \\
0,119\end{array}$ & $\begin{array}{l}0,028 \\
0,015 \\
0,015 \\
0,011\end{array}$ & $\begin{array}{l}97,23 \\
97,69 \\
97,46 \\
98,31\end{array}$ & $\begin{array}{r}-0,195 \\
0,037 \\
0,202 \\
-0,050\end{array}$ & $\begin{array}{l}0,217 \\
0,138 \\
0,197 \\
0,101\end{array}$ & $\begin{array}{l}0,234 \\
0,156 \\
0,215 \\
0,113\end{array}$ & $\begin{array}{l}0,085 \\
0,020 \\
0,080 \\
0,013\end{array}$ & $\begin{array}{l}91,08 \\
97,23 \\
88,08 \\
95,92\end{array}$ & $\begin{array}{r}-0,341 \\
0,149 \\
-0,410 \\
0,129\end{array}$ & $\begin{array}{l}0,265 \\
0,170 \\
0,307 \\
0,179\end{array}$ & $\begin{array}{l}0,279 \\
0,186 \\
0,321 \\
0,189\end{array}$ & $\begin{array}{l}0,187 \\
0,051 \\
0,262 \\
0,049\end{array}$ & $\begin{array}{l}81,23 \\
91,08 \\
79,46 \\
92,08\end{array}$ \\
\hline$\tau=0,5$ & $\pi_{c}=67,06 \%$ & $\begin{array}{l}\beta_{1} \\
\beta_{2} \\
\phi_{1} \\
\phi_{2}\end{array}$ & $\begin{array}{r}-0,211 \\
0,088 \\
0,128 \\
0,109\end{array}$ & $\begin{array}{l}0,276 \\
0,154 \\
0,229 \\
0,144\end{array}$ & $\begin{array}{l}0,291 \\
0,167 \\
0,239 \\
0,157\end{array}$ & $\begin{array}{l}0,121 \\
0,031 \\
0,069 \\
0,033\end{array}$ & $\begin{array}{l}90,54 \\
95,31 \\
94,31 \\
92,62 \\
\end{array}$ & $\begin{array}{r}-0,005 \\
0,007 \\
-0,016 \\
0,008\end{array}$ & $\begin{array}{l}0,074 \\
0,039 \\
0,068 \\
0,042\end{array}$ & $\begin{array}{l}0,079 \\
0,040 \\
0,069 \\
0,046\end{array}$ & $\begin{array}{l}0,006 \\
0,002 \\
0,005 \\
0,002\end{array}$ & $\begin{array}{l}96,85 \\
95,69 \\
95,54 \\
96,38\end{array}$ & $\begin{array}{r}-0,573 \\
0,174 \\
0,018 \\
0,111\end{array}$ & $\begin{array}{l}0,251 \\
0,197 \\
0,296 \\
0,203\end{array}$ & $\begin{array}{l}0,262 \\
0,211 \\
0,313 \\
0,215\end{array}$ & $\begin{array}{l}0,391 \\
0,069 \\
0,088 \\
0,054\end{array}$ & $\begin{array}{l}45,46 \\
90,15 \\
97,15 \\
94,23 \\
\end{array}$ \\
\hline$\tau$ & $62,90 \%$ & $\begin{array}{l}\beta_{1} \\
\beta_{2} \\
\phi_{1} \\
\phi_{2}\end{array}$ & $\begin{array}{r}0,160 \\
0,084 \\
-0,110 \\
-0,182 \\
\end{array}$ & $\begin{array}{l}0,285 \\
0,183 \\
0,295 \\
0,198\end{array}$ & $\begin{array}{l}0,298 \\
0,195 \\
0,308 \\
0,211\end{array}$ & $\begin{array}{l}0,107 \\
0,041 \\
0,099 \\
0,072\end{array}$ & $\begin{array}{l}94,08 \\
95,46 \\
95,54 \\
89,62 \\
\end{array}$ & $\begin{array}{r}0,101 \\
0,046 \\
-0,045 \\
-0,165 \\
\end{array}$ & $\begin{array}{l}0,286 \\
0,196 \\
0,302 \\
0,200\end{array}$ & $\begin{array}{l}0,304 \\
0,214 \\
0,321 \\
0,217\end{array}$ & $\begin{array}{l}0,092 \\
0,041 \\
0,093 \\
0,067 \\
\end{array}$ & $\begin{array}{l}95,77 \\
96,54 \\
96,62 \\
91,54 \\
\end{array}$ & $\begin{array}{r}-0,007 \\
0,005 \\
-0,006 \\
0,006 \\
\end{array}$ & $\begin{array}{l}0,139 \\
0,116 \\
0,103 \\
0,118\end{array}$ & $\begin{array}{l}0,154 \\
0,131 \\
0,115 \\
0,132\end{array}$ & $\begin{array}{l}0,019 \\
0,013 \\
0,011 \\
0,014\end{array}$ & $\begin{array}{l}97,69 \\
97,85 \\
97,69 \\
97,69 \\
\end{array}$ \\
\hline
\end{tabular}

Tabela C.11: Estimativas das Réplicas $n=300$ pelo Modelo M-splines (Frank)

\begin{tabular}{|c|c|c|c|c|c|c|c|c|c|c|c|c|c|c|c|c|c|}
\hline \multicolumn{18}{|c|}{ Cenário: $n=300, N=1300, \alpha_{t}=2, \lambda_{t}=2$ e $\alpha_{c}=\frac{1}{5}$} \\
\hline & & & \multicolumn{5}{|c|}{$\widetilde{\tau}=0,8$} & \multicolumn{5}{|c|}{$\tilde{\tau}=0,5$} & \multicolumn{5}{|c|}{$\widetilde{\tau}=0$} \\
\hline & & & Viés & EPMC & EPAM & EQM & $\mathrm{CP} \%$ & Viés & EPMC & EPAM & EQM & $\mathrm{CP} \%$ & Viés & EPMC & EPAM & EQM & $\mathrm{CP} \%$ \\
\hline \multirow{4}{*}{$\tau=0,8$} & \multirow{4}{*}{$\pi_{c}=36,18 \%$} & & 0,038 & 0,160 & 0,170 & 0,027 & 96,69 & $-0,214$ & 0,209 & 0,227 & 0,089 & 88,00 & $-0,419$ & 0,333 & 0,352 & 0,286 & 81,08 \\
\hline & & $\beta_{2}$ & 0,026 & 0,110 & 0,120 & 0,013 & 97,15 & 0,111 & 0,181 & 0,197 & 0,045 & 93,69 & 0,156 & 0,288 & 0,301 & 0,107 & 94,62 \\
\hline & & $\phi_{1}$ & 0,026 & 0,160 & 0,175 & 0,026 & 97,46 & 0,221 & 0,217 & 0,235 & 0,096 & 88,31 & $-0,408$ & 0,334 & 0,351 & 0,278 & 81,46 \\
\hline & & $\phi_{2}$ & 0,022 & 0,113 & 0,132 & 0,013 & 98,00 & $-0,039$ & 0,130 & 0,145 & 0,018 & 97,23 & 0,131 & 0,183 & 0,196 & 0,051 & 93,38 \\
\hline \multirow{4}{*}{$\tau=0,5$} & \multirow{4}{*}{$\pi_{c}=38,44 \%$} & $\beta_{1}$ & $-0,252$ & 0,278 & 0,288 & 0,141 & 88,08 & $-0,038$ & 0,153 & 0,159 & 0,025 & 96,15 & $-0,279$ & 0,284 & 0,301 & 0,158 & 87,69 \\
\hline & & $\beta_{2}$ & 0,121 & 0,151 & 0,164 & 0,037 & 92,46 & 0,005 & 0,113 & 0,115 & 0,013 & 96,46 & 0,108 & 0,213 & 0,229 & 0,057 & 95,23 \\
\hline & & $\phi_{1}$ & 0,113 & 0,274 & 0,287 & 0,088 & 95,77 & $-0,053$ & 0,171 & 0,172 & 0,032 & 95,00 & 0,023 & 0,307 & 0,322 & 0,095 & 97,08 \\
\hline & & $\phi_{2}$ & 0,100 & 0,170 & 0,187 & 0,039 & 95,15 & 0,003 & 0,130 & 0,131 & 0,017 & 96,46 & 0,114 & 0,216 & 0,228 & 0,060 & 94,77 \\
\hline \multirow{4}{*}{$\tau=0$} & \multirow{4}{*}{$\pi_{c}=42,90 \%$} & $\beta_{1}$ & 0,157 & 0,314 & 0,324 & 0,123 & 94,38 & 0,091 & 0,313 & 0,329 & 0,106 & 95,85 & $-0,030$ & 0,151 & 0,168 & 0,024 & 96,92 \\
\hline & & $\beta_{2}$ & 0,161 & 0,178 & 0,197 & 0,058 & 91,69 & 0,099 & 0,204 & 0,219 & 0,051 & 95,38 & 0,035 & 0,111 & 0,130 & 0,014 & 98,23 \\
\hline & & $\phi_{1}$ & $-0,042$ & 0,342 & 0,354 & 0,119 & 95,85 & $-0,057$ & 0,335 & 0,353 & 0,115 & 96,69 & 0,082 & 0,163 & 0,173 & 0,033 & 94,92 \\
\hline & & $\phi_{2}$ & $-0,163$ & 0,225 & 0,241 & 0,077 & 92,69 & $-0,173$ & 0,221 & 0,240 & 0,079 & 93,08 & $-0,030$ & 0,129 & 0,140 & 0,018 & 96,23 \\
\hline \multicolumn{18}{|c|}{ Cenário: $n=300, N=1300, \alpha_{t}=2, \lambda_{t}=2,5$ e $\alpha_{c}=\frac{1}{3}$} \\
\hline & & & \multicolumn{5}{|c|}{$\tilde{\tau}=0,8$} & \multicolumn{5}{|c|}{$\tilde{\tau}=0,5$} & \multicolumn{5}{|c|}{$\tilde{\tau}=0$} \\
\hline & & & Viés & EPMC & EPAM & EQM & $\mathrm{CP} \%$ & Viés & EPMC & EPAM & EQM & $\mathrm{CP} \%$ & Viés & EPMC & EPAM & EQM & $\mathrm{CP} \%$ \\
\hline \multirow{4}{*}{$\tau=0,8$} & \multirow{4}{*}{$\pi_{c}=70,62 \%$} & $\beta_{1}$ & 0,074 & 0,171 & 0,190 & 0,035 & 96,31 & $-0,185$ & 0,238 & 0,252 & 0,091 & 92,00 & $-0,343$ & 0,331 & 0,350 & 0,227 & 87,15 \\
\hline & & $\beta_{2}$ & 0,009 & 0,123 & 0,135 & 0,015 & 97,77 & $-0,157$ & 0,128 & 0,138 & 0,041 & 83,38 & 0,194 & 0,133 & 0,148 & 0,055 & 79,54 \\
\hline & & $\phi_{1}$ & 0,019 & 0,164 & 0,179 & 0,027 & 97,54 & 0,219 & 0,209 & 0,228 & 0,092 & 87,69 & $-0,412$ & 0,315 & 0,325 & 0,269 & 79,15 \\
\hline & & $\phi_{2}$ & 0,007 & 0,116 & 0,134 & 0,014 & 98,23 & $-0,040$ & 0,135 & 0,149 & 0,020 & 97,23 & 0,169 & 0,138 & 0,149 & 0,048 & 83,69 \\
\hline \multirow{4}{*}{$\tau=0,5$} & \multirow{4}{*}{$\pi_{c}=67,06 \%$} & $\beta_{1}$ & $-0,270$ & 0,348 & 0,363 & 0,194 & 92,15 & 0,018 & 0,142 & 0,160 & 0,020 & 97,08 & $-0,586$ & 0,321 & 0,334 & 0,446 & 62,69 \\
\hline & & $\beta_{2}$ & 0,112 & 0,278 & 0,291 & 0,090 & 95,31 & $-0,010$ & 0,092 & 0,105 & 0,009 & 97,15 & 0,205 & 0,218 & 0,233 & 0,090 & 89,38 \\
\hline & & $\phi_{1}$ & 0,114 & 0,486 & 0,500 & 0,249 & 95,54 & $-0,021$ & 0,135 & 0,154 & 0,019 & 96,85 & 0,235 & 0,314 & 0,327 & 0,154 & 91,00 \\
\hline & & $\phi_{2}$ & 0,101 & 0,186 & 0,200 & 0,045 & 94,77 & 0,009 & 0,095 & 0,106 & 0,009 & 96,92 & 0,122 & 0,221 & 0,233 & 0,064 & 94,00 \\
\hline \multirow{4}{*}{$\tau=0$} & \multirow{4}{*}{$\pi_{c}=62,90 \%$} & $\beta_{1}$ & 0,282 & 0,407 & 0,425 & 0,245 & 92,38 & 0,135 & 0,312 & 0,329 & 0,116 & 95,46 & $-0,034$ & 0,188 & 0,198 & 0,036 & 96,92 \\
\hline & & $\beta_{2}$ & 0,228 & 0,235 & 0,245 & 0,107 & 88,23 & 0,033 & 0,255 & 0,268 & 0,066 & 97,00 & 0,032 & 0,146 & 0,156 & 0,022 & 97,46 \\
\hline & & $\phi_{1}$ & $-0,048$ & 0,324 & 0,341 & 0,107 & 96,15 & $-0,060$ & 0,254 & 0,265 & 0,068 & 96,62 & 0,079 & 0,133 & 0,151 & 0,024 & 95,92 \\
\hline & & $\dot{\phi}_{2}$ & $-0,167$ & 0,213 & 0,223 & 0,073 & 92,31 & $-0,176$ & 0,196 & 0,207 & 0,069 & 90,54 & $-0,033$ & 0,121 & 0,134 & 0,016 & 97,31 \\
\hline
\end{tabular}


Tabela C.12: Estimativas das Réplicas $n=1000$ pelo Modelo M-splines (Frank)

\begin{tabular}{|c|c|c|c|c|c|c|c|c|c|c|c|c|c|c|c|c|c|}
\hline \multicolumn{18}{|c|}{ Cenário: $n=1000, N=1300, \alpha_{t}=2, \lambda_{t}=2$ e $\alpha_{c}=\frac{1}{5}$} \\
\hline & & & \multicolumn{5}{|c|}{$\widetilde{\tau}=0,8$} & \multicolumn{5}{|c|}{$\widetilde{\tau}=0,5$} & \multicolumn{5}{|c|}{$\tilde{\tau}=0$} \\
\hline & & & Viés & EPMC & EPAM & EQM & $\mathrm{CP} \%$ & Viés & EPMC & EPAM & EQM & $\mathrm{CP} \%$ & Viés & EPMC & EPAM & EQM & $\mathrm{CP} \%$ \\
\hline$\tau=0,8$ & $\pi_{c}=36,18 \%$ & $\begin{array}{l}\beta_{1} \\
\beta_{2} \\
\phi_{1} \\
\phi_{2} \\
\end{array}$ & $\begin{array}{r}0.013 \\
0.013 \\
0.012 \\
-0.002 \\
\end{array}$ & $\begin{array}{l}0.150 \\
0.110 \\
0.145 \\
0.110 \\
\end{array}$ & $\begin{array}{l}0.165 \\
0.128 \\
0.160 \\
0.120 \\
\end{array}$ & $\begin{array}{l}0.023 \\
0.012 \\
0.021 \\
0.012 \\
\end{array}$ & $\begin{array}{l}97.46 \\
98.15 \\
97.46 \\
97.31 \\
\end{array}$ & $\begin{array}{r}-0.188 \\
0.039 \\
0.206 \\
-0.048 \\
\end{array}$ & $\begin{array}{l}0.195 \\
0.171 \\
0.213 \\
0.117 \\
\end{array}$ & $\begin{array}{l}0.214 \\
0.182 \\
0.230 \\
0.134 \\
\end{array}$ & $\begin{array}{l}0.073 \\
0.031 \\
0.088 \\
0.016 \\
\end{array}$ & $\begin{array}{l}90.15 \\
96.62 \\
89.46 \\
96.92 \\
\end{array}$ & $\begin{array}{r}-0.334 \\
-0.112 \\
-0.402 \\
0.134 \\
\end{array}$ & $\begin{array}{l}0.269 \\
0.162 \\
0.311 \\
0.182 \\
\end{array}$ & $\begin{array}{l}0.279 \\
0.181 \\
0.328 \\
0.194 \\
\end{array}$ & $\begin{array}{l}0.184 \\
0.039 \\
0.258 \\
0.051 \\
\end{array}$ & $\begin{array}{l}81.62 \\
94.46 \\
80.85 \\
91.62 \\
\end{array}$ \\
\hline$\tau=0,5$ & $\pi_{c}=38,44 \%$ & $\begin{array}{l}\beta_{1} \\
\beta_{2} \\
\phi_{1} \\
\phi_{2} \\
\end{array}$ & $\begin{array}{r}-0.149 \\
0.073 \\
0.122 \\
0.106 \\
\end{array}$ & $\begin{array}{l}0.256 \\
0.136 \\
0.254 \\
0.167 \\
\end{array}$ & $\begin{array}{l}0.274 \\
0.153 \\
0.266 \\
0.181 \\
\end{array}$ & $\begin{array}{l}0.088 \\
0.024 \\
0.079 \\
0.039 \\
\end{array}$ & $\begin{array}{l}94.62 \\
95.77 \\
94.85 \\
93.85 \\
\end{array}$ & $\begin{array}{r}-0.009 \\
0.004 \\
-0.020 \\
0.005 \\
\end{array}$ & $\begin{array}{l}0.056 \\
0.047 \\
0.060 \\
0.053\end{array}$ & $\begin{array}{l}0.061 \\
0.052 \\
0.066 \\
0.055\end{array}$ & $\begin{array}{l}0.003 \\
0.002 \\
0.004 \\
0.003 \\
\end{array}$ & $\begin{array}{l}95.85 \\
96.38 \\
95.38 \\
95.00 \\
\end{array}$ & $\begin{array}{r}-0.218 \\
0.079 \\
0.024 \\
0.115 \\
\end{array}$ & $\begin{array}{l}0.271 \\
0.207 \\
0.296 \\
0.203\end{array}$ & $\begin{array}{l}0.288 \\
0.222 \\
0.314 \\
0.216 \\
\end{array}$ & $\begin{array}{l}0.121 \\
0.049 \\
0.088 \\
0.054 \\
\end{array}$ & $\begin{array}{l}91.54 \\
96.08 \\
97.23 \\
94.54 \\
\end{array}$ \\
\hline$\tau=0$ & $=42,90 \%$ & $\begin{array}{l}\beta_{1} \\
\beta_{2} \\
\phi_{1} \\
\phi_{2}\end{array}$ & $\begin{array}{r}0.095 \\
0.090 \\
-0.084 \\
-0.165\end{array}$ & $\begin{array}{l}0.313 \\
0.192 \\
0.309 \\
0.207\end{array}$ & $\begin{array}{l}0.327 \\
0.206 \\
0.326 \\
0.219\end{array}$ & $\begin{array}{l}0.107 \\
0.045 \\
0.103 \\
0.070\end{array}$ & $\begin{array}{l}96.31 \\
95.77 \\
96.62 \\
90.62 \\
\end{array}$ & $\begin{array}{r}0.059 \\
0.074 \\
-0.047 \\
-0.166\end{array}$ & $\begin{array}{l}0.227 \\
0.154 \\
0.314 \\
0.208\end{array}$ & $\begin{array}{l}0.244 \\
0.171 \\
0.326 \\
0.221\end{array}$ & $\begin{array}{l}0.055 \\
0.029 \\
0.101 \\
0.071\end{array}$ & $\begin{array}{l}97.15 \\
96.23 \\
97.08 \\
91.54\end{array}$ & $\begin{array}{r}-0.004 \\
0.003 \\
-0.008 \\
0.004\end{array}$ & $\begin{array}{l}0.125 \\
0.089 \\
0.100 \\
0.115\end{array}$ & $\begin{array}{l}0.141 \\
0.100 \\
0.114 \\
0.134\end{array}$ & $\begin{array}{l}0.016 \\
0.008 \\
0.010 \\
0.013\end{array}$ & $\begin{array}{l}98.15 \\
97.85 \\
98.38 \\
98.31\end{array}$ \\
\hline \multicolumn{18}{|c|}{ Cenário: $n=1000, N=1300, \alpha_{t}=2, \lambda_{t}=2,5$ e $\alpha_{c}=\frac{1}{3}$} \\
\hline & & & \multicolumn{5}{|c|}{$\tilde{\tau}=0,8$} & \multicolumn{5}{|c|}{$\bar{\tau} \tilde{\tau}=0,5$} & \multicolumn{5}{|c|}{$\tilde{\tau}=0$} \\
\hline & & & Viés & EPMC & EPAM & EQM & $\mathrm{CP} \%$ & Viés & EPMC & EPAM & EQM & $\mathrm{CP} \%$ & Viés & EPMC & EPAM & EQM & $\mathrm{CP} \%$ \\
\hline$\tau=0,8$ & $\pi_{c}=70,62 \%$ & $\begin{array}{l}\beta_{1} \\
\beta_{2} \\
\phi_{1} \\
\phi_{2}\end{array}$ & $\begin{array}{r}0.008 \\
0.006 \\
0.008 \\
-0.008\end{array}$ & $\begin{array}{l}0.167 \\
0.123 \\
0.123 \\
0.102\end{array}$ & $\begin{array}{l}0.180 \\
0.133 \\
0.141 \\
0.120\end{array}$ & $\begin{array}{l}0.028 \\
0.015 \\
0.015 \\
0.010\end{array}$ & $\begin{array}{l}97.62 \\
97.69 \\
98.23 \\
98.85\end{array}$ & $\begin{array}{r}-0.184 \\
0.043 \\
0.211 \\
-0.045\end{array}$ & $\begin{array}{l}0.218 \\
0.139 \\
0.198 \\
0.102\end{array}$ & $\begin{array}{l}0.232 \\
0.158 \\
0.217 \\
0.116\end{array}$ & $\begin{array}{l}0.081 \\
0.021 \\
0.084 \\
0.012\end{array}$ & $\begin{array}{l}91.62 \\
97.23 \\
87.92 \\
97.23\end{array}$ & $\begin{array}{r}-0.343 \\
0.148 \\
-0.412 \\
0.128\end{array}$ & $\begin{array}{l}0.263 \\
0.169 \\
0.305 \\
0.178\end{array}$ & $\begin{array}{l}0.274 \\
0.183 \\
0.321 \\
0.193\end{array}$ & $\begin{array}{l}0.187 \\
0.050 \\
0.263 \\
0.048\end{array}$ & $\begin{array}{l}80.54 \\
90.00 \\
80.00 \\
92.46\end{array}$ \\
\hline$\tau=0,5$ & $\pi_{c}=67,06 \%$ & $\begin{array}{l}\beta_{1} \\
\beta_{2} \\
\phi_{1} \\
\phi_{2}\end{array}$ & $\begin{array}{r}-0.237 \\
0.073 \\
0.106 \\
0.096 \\
\end{array}$ & $\begin{array}{l}0.276 \\
0.154 \\
0.230 \\
0.144\end{array}$ & $\begin{array}{l}0.291 \\
0.167 \\
0.243 \\
0.155\end{array}$ & $\begin{array}{l}0.132 \\
0.029 \\
0.064 \\
0.030\end{array}$ & $\begin{array}{l}89.69 \\
95.92 \\
95.54 \\
93.85 \\
\end{array}$ & $\begin{array}{r}-0.010 \\
0.005 \\
-0.020 \\
0.006\end{array}$ & $\begin{array}{l}0.076 \\
0.040 \\
0.070 \\
0.043\end{array}$ & $\begin{array}{l}0.085 \\
0.045 \\
0.077 \\
0.046\end{array}$ & $\begin{array}{l}0.006 \\
0.002 \\
0.005 \\
0.002\end{array}$ & $\begin{array}{l}96.08 \\
96.38 \\
95.46 \\
96.00 \\
\end{array}$ & $\begin{array}{r}-0.575 \\
0.172 \\
0.017 \\
0.109\end{array}$ & $\begin{array}{l}0.253 \\
0.199 \\
0.299 \\
0.206\end{array}$ & $\begin{array}{l}0.264 \\
0.214 \\
0.318 \\
0.222 \\
\end{array}$ & $\begin{array}{l}0.395 \\
0.069 \\
0.090 \\
0.054\end{array}$ & $\begin{array}{l}43.92 \\
90.23 \\
97.23 \\
95.31 \\
\end{array}$ \\
\hline$\tau=0$ & $\pi_{c}=62,90 \%$ & $\begin{array}{l}\beta_{1} \\
\beta_{2} \\
\phi_{1} \\
\phi_{2} \\
\end{array}$ & $\begin{array}{r}0.187 \\
0.101 \\
-0.082 \\
-0.163 \\
\end{array}$ & $\begin{array}{l}0.300 \\
0.193 \\
0.310 \\
0.208 \\
\end{array}$ & $\begin{array}{l}0.316 \\
0.210 \\
0.323 \\
0.226\end{array}$ & $\begin{array}{l}0.125 \\
0.047 \\
0.103 \\
0.070\end{array}$ & $\begin{array}{l}93.77 \\
95.85 \\
96.15 \\
92.92 \\
\end{array}$ & $\begin{array}{r}0.095 \\
0.041 \\
-0.052 \\
-0.170 \\
\end{array}$ & $\begin{array}{l}0.290 \\
0.199 \\
0.306 \\
0.203\end{array}$ & $\begin{array}{l}0.305 \\
0.216 \\
0.322 \\
0.222 \\
\end{array}$ & $\begin{array}{l}0.093 \\
0.041 \\
0.096 \\
0.070 \\
\end{array}$ & $\begin{array}{l}95.77 \\
97.00 \\
96.77 \\
91.62 \\
\end{array}$ & $\begin{array}{r}-0.004 \\
0.003 \\
-0.008 \\
0.004 \\
\end{array}$ & $\begin{array}{l}0.129 \\
0.107 \\
0.104 \\
0.119 \\
\end{array}$ & $\begin{array}{l}0.147 \\
0.121 \\
0.116 \\
0.137 \\
\end{array}$ & $\begin{array}{l}0.017 \\
0.011 \\
0.011 \\
0.014 \\
\end{array}$ & $\begin{array}{l}98.15 \\
98.15 \\
97.85 \\
98.31 \\
\end{array}$ \\
\hline
\end{tabular}

Tabela C.13: Estimativas das Réplicas TSAQV $n=300$ e $n=1000$ pelo Modelo Piecewise (Clayton)

\begin{tabular}{|c|c|c|c|c|c|c|c|c|c|c|c|c|c|c|c|}
\hline \multicolumn{16}{|c|}{ Cenário: $n=300, N=1300, \alpha_{t}=2, \lambda_{t}=2, \tau=0,20$ e $\alpha_{c}=\frac{1}{5}$} \\
\hline & \multicolumn{5}{|c|}{$\widetilde{\tau}=0,10$} & \multicolumn{5}{|c|}{$\widetilde{\tau}=0,15$} & \multicolumn{5}{|c|}{$\widetilde{\tau}=0,20$} \\
\hline & Viés & EPMC & EPAM & EQM & $\mathrm{CP} \%$ & Viés & EPMC & EPAM & EQM & $\mathrm{CP} \%$ & Viés & EPMC & EPAM & EQM & $\mathrm{CP} \%$ \\
\hline$\beta_{1}$ & 0,067 & 0,119 & 0,132 & 0,019 & 93,77 & 0,067 & 0,119 & 0,135 & 0,019 & 94,85 & 0,041 & 0,088 & 0,106 & 0,009 & 96,69 \\
\hline$\beta_{2}$ & $-0,007$ & 0,090 & 0,104 & 0,008 & 96,69 & $-0,006$ & 0,093 & 0,107 & 0,009 & 96,62 & $-0,007$ & 0,040 & 0,054 & 0,002 & 97,85 \\
\hline$\phi_{1}$ & $-0,036$ & 0,109 & 0,128 & 0,013 & 96,15 & $-0,035$ & 0,112 & 0,130 & 0,014 & 96,54 & $-0,007$ & 0,088 & 0,100 & 0,008 & 96,38 \\
\hline \multirow[t]{2}{*}{$\phi_{2}$} & 0,011 & 0,091 & 0,106 & 0,008 & 96,69 & 0,011 & 0,115 & 0,128 & 0,013 & 96,31 & 0,006 & 0,071 & 0,085 & 0,005 & 97,23 \\
\hline & \multicolumn{5}{|c|}{$\tilde{\tau}=0,25$} & \multicolumn{5}{|c|}{$\widetilde{\tau}=0,30$} & \multicolumn{5}{|c|}{$\widetilde{\tau}=0,35$} \\
\hline$\beta_{1}$ & 0,024 & 0,129 & 0,142 & 0,017 & 96,54 & $-0,001$ & 0,123 & 0,133 & 0,015 & 97,46 & $-0,025$ & 0,129 & 0,142 & 0,017 & 97,92 \\
\hline$\beta_{2}$ & $-0,001$ & 0,087 & 0,102 & 0,008 & 97,08 & 0,000 & 0,089 & 0,105 & 0,008 & 97,23 & $-0,001$ & 0,100 & 0,119 & 0,010 & 97,31 \\
\hline$\phi_{1}$ & 0,035 & 0,118 & 0,128 & 0,015 & 94,92 & 0,069 & 0,119 & 0,132 & 0,019 & 94,23 & 0,100 & 0,128 & 0,142 & 0,026 & 91,62 \\
\hline$\phi_{2}$ & 0,010 & 0,131 & 0,143 & 0,017 & 96,23 & 0,007 & 0,140 & 0,158 & 0,020 & 96,77 & 0,000 & 0,166 & 0,184 & 0,028 & 96,69 \\
\hline & \multicolumn{5}{|c|}{$\widetilde{\tau}=0,40$} & \multicolumn{5}{|c|}{$\widetilde{\tau}=0,45$} & \multicolumn{5}{|c|}{$\widetilde{\tau}=0,50$} \\
\hline$\beta_{1}$ & $-0,047$ & 0,161 & 0,175 & 0,028 & 98,08 & $-0,074$ & 0,276 & 0,291 & 0,082 & 97,77 & $-0,099$ & 0,300 & 0,311 & 0,100 & 97,77 \\
\hline$\beta_{2}$ & 0,001 & 0,125 & 0,136 & 0,016 & 96,08 & $-0,003$ & 0,159 & 0,178 & 0,025 & 96,38 & $-0,001$ & 0,189 & 0,201 & 0,036 & 96,08 \\
\hline$\phi_{1}$ & 0,141 & 0,144 & 0,158 & 0,041 & 88,23 & 0,175 & 0,168 & 0,182 & 0,059 & 87,15 & 0,222 & 0,180 & 0,194 & 0,082 & 81,69 \\
\hline$\phi_{2}$ & $-0,004$ & 0,180 & 0,192 & 0,032 & 95,92 & $-0,015$ & 0,194 & 0,205 & 0,038 & 95,31 & $-0,019$ & 0,202 & 0,212 & 0,041 & 95,69 \\
\hline \multicolumn{16}{|c|}{ Cenário: $n=1000, N=500, \alpha_{t}=2, \lambda_{t}=2, \tau=0,20$ e $\alpha_{c}=\frac{1}{5}$} \\
\hline & Viés & EPMC & EPAM & EQM & $\mathrm{CP} \%$ & Viés & EPMC & EPAM & EQM & $\mathrm{CP} \%$ & Viés & EPMC & EPAM & EQM & $\mathrm{CP} \%$ \\
\hline & \multicolumn{5}{|c|}{$\widetilde{\tau}=0,10$} & \multicolumn{5}{|c|}{$\widetilde{\tau}=0,15$} & \multicolumn{5}{|c|}{$\widetilde{\tau}=0,20$} \\
\hline$\beta_{1}$ & 0,038 & 0,020 & 0,033 & 0,002 & 91,54 & 0,018 & 0,026 & 0,036 & 0,001 & 97,23 & $-0,005$ & 0,017 & 0,035 & 0,000 & 98,54 \\
\hline$\beta_{2}$ & $-0,015$ & 0,051 & 0,065 & 0,003 & 97,46 & $-0,012$ & 0,061 & 0,076 & 0,004 & 97,00 & $-0,010$ & 0,009 & 0,019 & 0,000 & 98,46 \\
\hline$\phi_{1}$ & $-0,001$ & 0,109 & 0,128 & 0,012 & 97,00 & 0,030 & 0,110 & 0,121 & 0,013 & 95,85 & 0,061 & 0,012 & 0,029 & 0,004 & 45,54 \\
\hline$\phi_{2}$ & 0,022 & 0,121 & 0,136 & 0,015 & 95,85 & 0,021 & 0,112 & 0,129 & 0,013 & 96,62 & 0,016 & 0,027 & 0,041 & 0,001 & 98,08 \\
\hline & \multicolumn{5}{|c|}{$\widetilde{\tau}=0,25$} & \multicolumn{5}{|c|}{$\widetilde{\tau}=0,30$} & \multicolumn{5}{|c|}{$\widetilde{\tau}=0,35$} \\
\hline$\beta_{1}$ & $-0,028$ & 0,032 & 0,050 & 0,002 & 98,54 & $-0,050$ & 0,042 & 0,060 & 0,004 & 98,54 & $-0,075$ & 0,053 & 0,072 & 0,008 & 98,54 \\
\hline$\beta_{2}$ & $-0,008$ & 0,081 & 0,093 & 0,007 & 96,38 & $-0,007$ & 0,088 & 0,104 & 0,008 & 97,15 & $-0,004$ & 0,099 & 0,109 & 0,010 & 96,08 \\
\hline$\phi_{1}$ & 0,091 & 0,080 & 0,098 & 0,015 & 90,54 & 0,121 & 0,089 & 0,099 & 0,023 & 80,54 & 0,158 & 0,106 & 0,117 & 0,036 & 77,92 \\
\hline$\phi_{2}$ & 0,012 & 0,092 & 0,105 & 0,009 & 96,31 & 0,007 & 0,105 & 0,121 & 0,011 & 96,85 & 0,003 & 0,115 & 0,130 & 0,013 & 96,31 \\
\hline & \multicolumn{5}{|c|}{$\widetilde{\tau}=0,40$} & \multicolumn{5}{|c|}{$\widetilde{\tau}=0,45$} & \multicolumn{5}{|c|}{$\widetilde{\tau}=0,50$} \\
\hline$\beta_{1}$ & $-0,098$ & 0,076 & 0,086 & 0,015 & 98,46 & $-0,097$ & 0,106 & 0,119 & 0,021 & 98,54 & $-0,133$ & 0,174 & 0,191 & 0,048 & 98,38 \\
\hline$\beta_{2}$ & $-0,007$ & 0,110 & 0,120 & 0,012 & 96,54 & 0,001 & 0,115 & 0,131 & 0,013 & 96,38 & $-0,007$ & 0,121 & 0,131 & 0,015 & 95,85 \\
\hline$\beta_{2}$ & 0,188 & 0,120 & 0,130 & 0,050 & 73,31 & 0,224 & 0,125 & 0,140 & 0,066 & 67,31 & 0,264 & 0,137 & 0,149 & 0,088 & 61,23 \\
\hline$\beta_{2}$ & $-0,006$ & 0,133 & 0,148 & 0,018 & 96,62 & $-0,017$ & 0,149 & 0,163 & 0,022 & 95,54 & $-0,033$ & 0,154 & 0,167 & 0,025 & 95,54 \\
\hline
\end{tabular}


Tabela C.14: Estimativas das Réplicas TSAQV $n=300$ e $n=1000$ pelo Modelo Piecewise (Frank)

\begin{tabular}{|c|c|c|c|c|c|c|c|c|c|c|c|c|c|c|c|}
\hline \multicolumn{16}{|c|}{ Cenário: $n=300, N=1300, \alpha_{t}=2, \lambda_{t}=2, \tau=0,20$ e $\alpha_{c}=\frac{1}{5}$} \\
\hline & \multicolumn{5}{|c|}{$\widetilde{\tau}=0,10$} & \multicolumn{5}{|c|}{$\widetilde{\tau}=0,15$} & \multicolumn{5}{|c|}{$\widetilde{\tau}=0,20$} \\
\hline & Viés & EPMC & EPAM & EQM & $\mathrm{CP} \%$ & Viés & EPMC & EPAM & EQM & $\mathrm{CP} \%$ & Viés & EPMC & EPAM & EQM & $\mathrm{CP} \%$ \\
\hline$\beta_{1}$ & 0,065 & 0,208 & 0,221 & 0,047 & 95,31 & 0,031 & 0,214 & 0,229 & 0,047 & 96,69 & 0,023 & 0,171 & 0,189 & 0,030 & 96,92 \\
\hline$\beta_{2}$ & $-0,008$ & 0,114 & 0,126 & 0,013 & 96,46 & $-0,012$ & 0,116 & 0,134 & 0,014 & 97,08 & $-0,002$ & 0,098 & 0,115 & 0,010 & 97,31 \\
\hline$\phi_{1}$ & $-0,037$ & 0,228 & 0,243 & 0,053 & 95,62 & $-0,017$ & 0,216 & 0,233 & 0,047 & 96,38 & 0,034 & 0,153 & 0,168 & 0,025 & 95,77 \\
\hline$\phi_{2}$ & 0,010 & 0,124 & 0,143 & 0,015 & 96,85 & 0,001 & 0,118 & 0,133 & 0,014 & 96,77 & 0,007 & 0,106 & 0,117 & 0,011 & 96,23 \\
\hline & \multicolumn{5}{|c|}{$\widetilde{\tau}=0,25$} & \multicolumn{5}{|c|}{$\widetilde{\tau}=0,30$} & \multicolumn{5}{|c|}{$\widetilde{\tau}=0,35$} \\
\hline$\beta_{1}$ & 0,001 & 0,189 & 0,203 & 0,036 & 96,85 & $-0,034$ & 0,200 & 0,219 & 0,041 & 97,54 & $-0,055$ & 0,209 & 0,221 & 0,047 & 98,00 \\
\hline$\beta_{2}$ & 0,001 & 0,107 & 0,122 & 0,011 & 95,92 & $-0,006$ & 0,111 & 0,123 & 0,012 & 96,46 & $-0,005$ & 0,121 & 0,137 & 0,015 & 96,46 \\
\hline$\phi_{1}$ & 0,069 & 0,132 & 0,146 & 0,022 & 94,46 & 0,094 & 0,121 & 0,131 & 0,023 & 90,85 & 0,135 & 0,140 & 0,159 & 0,038 & 90,08 \\
\hline$\phi_{2}$ & 0,006 & 0,125 & 0,135 & 0,016 & 95,38 & $-0,006$ & 0,109 & 0,128 & 0,012 & 97,15 & $-0,011$ & 0,129 & 0,140 & 0,017 & 96,00 \\
\hline & \multicolumn{5}{|c|}{$\widetilde{\tau}=0,40$} & \multicolumn{5}{|c|}{$\widetilde{\tau}=0,45$} & \multicolumn{5}{|c|}{$\widetilde{\tau}=0,50$} \\
\hline$\beta_{1}$ & $-0,071$ & 0,247 & 0,263 & 0,066 & 97,77 & $-0,095$ & 0,265 & 0,284 & 0,079 & 97,77 & $-0,119$ & 0,271 & 0,283 & 0,088 & 97,92 \\
\hline$\beta_{2}$ & $-0,001$ & 0,159 & 0,170 & 0,025 & 95,46 & 0,001 & 0,154 & 0,169 & 0,024 & 96,00 & 0,003 & 0,194 & 0,206 & 0,038 & 95,38 \\
\hline$\phi_{1}$ & 0,176 & 0,148 & 0,161 & 0,053 & 84,15 & 0,225 & 0,193 & 0,211 & 0,088 & 85,08 & 0,274 & 0,145 & 0,160 & 0,096 & 63,00 \\
\hline$\phi_{2}$ & $-0,013$ & 0,177 & 0,192 & 0,031 & 95,77 & $-0,017$ & 0,163 & 0,182 & 0,027 & 96,15 & $-0,022$ & 0,186 & 0,200 & 0,035 & 95,38 \\
\hline \multicolumn{16}{|c|}{ Cenário: $n=1000, N=1300, \alpha_{t}=2, \lambda_{t}=2, \tau=0,20$ e $\alpha_{c}=\frac{1}{5}$} \\
\hline & Viés & EPMC & EPAM & EQM & $\mathrm{CP} \%$ & Viés & EPMC & EPAM & EQM & $\mathrm{CP} \%$ & Viés & EPMC & EPAM & EQM & $\mathrm{CP} \%$ \\
\hline & \multicolumn{5}{|c|}{$\widetilde{\tau}=0,10$} & \multicolumn{5}{|c|}{$\widetilde{\tau}=0,15$} & \multicolumn{5}{|c|}{$\widetilde{\tau}=0,20$} \\
\hline$\beta_{1}$ & 0,038 & 0,021 & 0,034 & 0,002 & 92,46 & 0,018 & 0,026 & 0,044 & 0,001 & 98,31 & $-0,005$ & 0,015 & 0,027 & 0,000 & 98,46 \\
\hline$\beta_{2}$ & $-0,015$ & 0,053 & 0,065 & 0,003 & 97,38 & $-0,012$ & 0,071 & 0,088 & 0,005 & 97,46 & $-0,011$ & 0,012 & 0,028 & 0,000 & 98,54 \\
\hline$\phi_{1}$ & $-0,002$ & 0,114 & 0,129 & 0,013 & 96,77 & 0,029 & 0,109 & 0,124 & 0,013 & 96,38 & 0,060 & 0,014 & 0,033 & 0,004 & 69,15 \\
\hline$\phi_{2}$ & 0,021 & 0,126 & 0,145 & 0,016 & 96,46 & 0,020 & 0,111 & 0,122 & 0,013 & 96,00 & 0,017 & 0,079 & 0,090 & 0,007 & 96,62 \\
\hline & \multicolumn{5}{|c|}{$\widetilde{\tau}=0,25$} & \multicolumn{5}{|c|}{$\widetilde{\tau}=0,30$} & \multicolumn{5}{|c|}{$\widetilde{\tau}=0,35$} \\
\hline$\beta_{1}$ & $-0,029$ & 0,034 & 0,052 & 0,002 & 98,54 & $-0,051$ & 0,046 & 0,060 & 0,005 & 98,46 & $-0,079$ & 0,056 & 0,073 & 0,009 & 98,54 \\
\hline$\beta_{2}$ & $-0,009$ & 0,081 & 0,094 & 0,007 & 96,85 & $-0,009$ & 0,091 & 0,105 & 0,008 & 96,85 & $-0,013$ & 0,103 & 0,119 & 0,011 & 96,92 \\
\hline$\phi_{1}$ & 0,091 & 0,080 & 0,091 & 0,015 & 86,00 & 0,119 & 0,092 & 0,107 & 0,023 & 84,54 & 0,148 & 0,111 & 0,127 & 0,034 & 83,08 \\
\hline$\phi_{2}$ & 0,011 & 0,103 & 0,120 & 0,011 & 96,92 & 0,005 & 0,113 & 0,124 & 0,013 & 95,85 & $-0,007$ & 0,129 & 0,148 & 0,017 & 96,92 \\
\hline & \multicolumn{5}{|c|}{$\widetilde{\tau}=0,40$} & \multicolumn{5}{|c|}{$\widetilde{\tau}=0,45$} & \multicolumn{5}{|c|}{$\widetilde{\tau}=0,50$} \\
\hline$\beta_{1}$ & $-0,093$ & 0,075 & 0,091 & 0,014 & 98,54 & $-0,101$ & 0,106 & 0,121 & 0,021 & 98,38 & $-0,123$ & 0,173 & 0,184 & 0,045 & 98,46 \\
\hline$\beta_{2}$ & 0,001 & 0,099 & 0,117 & 0,010 & 97,31 & $-0,003$ & 0,110 & 0,128 & 0,012 & 96,85 & 0,000 & 0,132 & 0,146 & 0,017 & 96,46 \\
\hline$\phi_{1}$ & 0,197 & 0,117 & 0,133 & 0,052 & 72,77 & 0,220 & 0,119 & 0,132 & 0,063 & 65,15 & 0,272 & 0,133 & 0,152 & 0,092 & 59,77 \\
\hline$\phi_{2}$ & 0,003 & 0,128 & 0,143 & 0,016 & 96,92 & $-0,022$ & 0,145 & 0,161 & 0,022 & 96,46 & $-0,024$ & 0,182 & 0,201 & 0,034 & 96,00 \\
\hline
\end{tabular}


Tabela C.15: Estimativas das Réplicas TSAQV $n=300$ e $n=1000-$ M-spline (Clayton)

\begin{tabular}{|c|c|c|c|c|c|c|c|c|c|c|c|c|c|c|c|}
\hline \multicolumn{16}{|c|}{ Cenário: $n=300, N=1300, \alpha_{t}=2, \lambda_{t}=2, \tau=0,20$ e $\alpha_{c}=\frac{1}{5}$} \\
\hline & \multicolumn{5}{|c|}{$\widetilde{\tau}=0,10$} & \multicolumn{5}{|c|}{$\widetilde{\tau}=0,15$} & \multicolumn{5}{|c|}{$\tilde{\tau}=0,20$} \\
\hline & Viés & EPMC & EPAM & EQM & $\mathrm{CP} \%$ & Viés & EPMC & EPAM & EQM & $\mathrm{CP} \%$ & Viés & EPMC & EPAM & EQM & $\mathrm{CP} \%$ \\
\hline$\beta_{1}$ & 0,020 & 0,133 & 0,143 & 0,018 & 96,46 & 0,018 & 0,097 & 0,109 & 0,010 & 96,92 & $-0,027$ & 0,060 & 0,076 & 0,004 & 98,38 \\
\hline$\beta_{2}$ & $-0,005$ & 0,093 & 0,105 & 0,009 & 96,85 & 0,010 & 0,072 & 0,088 & 0,005 & 97,46 & 0,007 & 0,031 & 0,049 & 0,001 & 98,38 \\
\hline$\phi_{1}$ & 0,029 & 0,133 & 0,150 & 0,019 & 96,15 & 0,017 & 0,093 & 0,104 & 0,009 & 96,23 & $-0,057$ & 0,064 & 0,078 & 0,007 & 93,77 \\
\hline \multirow[t]{2}{*}{$\phi_{2}$} & $-0,039$ & 0,084 & 0,100 & 0,009 & 96,38 & $-0,023$ & 0,091 & 0,107 & 0,009 & 96,54 & $-0,012$ & 0,045 & 0,058 & 0,002 & 97,38 \\
\hline & \multicolumn{5}{|c|}{$\widetilde{\tau}=0,25$} & \multicolumn{5}{|c|}{$\widetilde{\tau}=0,30$} & \multicolumn{5}{|c|}{$\widetilde{\tau}=0,35$} \\
\hline$\beta_{1}$ & 0,064 & 0,113 & 0,124 & 0,017 & 94,15 & 0,081 & 0,126 & 0,143 & 0,022 & 93,92 & 0,132 & 0,129 & 0,143 & 0,034 & 87,54 \\
\hline$\beta_{2}$ & 0,001 & 0,092 & 0,106 & 0,008 & 96,77 & 0,005 & 0,103 & 0,121 & 0,011 & 97,31 & 0,006 & 0,108 & 0,126 & 0,012 & 96,85 \\
\hline$\phi_{1}$ & 0,022 & 0,090 & 0,107 & 0,009 & 96,69 & 0,046 & 0,093 & 0,109 & 0,011 & 95,38 & $-0,030$ & 0,100 & 0,113 & 0,011 & 96,38 \\
\hline$\phi_{2}$ & $-0,027$ & 0,083 & 0,097 & 0,008 & 96,77 & $-0,049$ & 0,101 & 0,119 & 0,013 & 96,38 & $-0,046$ & 0,105 & 0,115 & 0,013 & 95,38 \\
\hline & \multicolumn{5}{|c|}{$\widetilde{\tau}=0,40$} & \multicolumn{5}{|c|}{$\widetilde{\tau}=0,45$} & \multicolumn{5}{|c|}{$\widetilde{\tau}=0,50$} \\
\hline$\beta_{1}$ & 0,156 & 0,133 & 0,143 & 0,042 & 82,62 & $-0,111$ & 0,141 & 0,159 & 0,032 & 98,46 & 0,192 & 0,154 & 0,172 & 0,061 & 84,00 \\
\hline$\beta_{2}$ & 0,012 & 0,112 & 0,123 & 0,013 & 96,54 & 0,009 & 0,121 & 0,134 & 0,015 & 96,23 & 0,013 & 0,136 & 0,149 & 0,019 & 96,15 \\
\hline$\phi_{1}$ & $-0,024$ & 0,110 & 0,128 & 0,013 & 96,46 & 0,042 & 0,121 & 0,134 & 0,016 & 96,00 & 0,011 & 0,135 & 0,148 & 0,018 & 96,31 \\
\hline$\phi_{2}$ & $-0,033$ & 0,116 & 0,130 & 0,015 & 95,85 & 0,007 & 0,122 & 0,133 & 0,015 & 95,85 & 0,012 & 0,134 & 0,151 & 0,018 & 96,69 \\
\hline \multicolumn{16}{|c|}{ Cenário: $n=1000, N=1300, \alpha_{t}=2, \lambda_{t}=2, \tau=0,20$ e $\alpha_{c}=\frac{1}{5}$} \\
\hline & Viés & EPMC & EPAM & EQM & $\mathrm{CP} \%$ & Viés & EPMC & EPAM & EQM & $\mathrm{CP} \%$ & Viés & EPMC & EPAM & EQM & $\mathrm{CP} \%$ \\
\hline & \multicolumn{5}{|c|}{$\widetilde{\tau}=0,10$} & \multicolumn{5}{|c|}{$\widetilde{\tau}=0,15$} & \multicolumn{5}{|c|}{$\widetilde{\tau}=0,20$} \\
\hline$\beta_{1}$ & 0,027 & 0,100 & 0,114 & 0,011 & 96,85 & 0,014 & 0,096 & 0,107 & 0,009 & 96,77 & $-0,019$ & 0,030 & 0,041 & 0,001 & 98,54 \\
\hline$\beta_{2}$ & $-0,004$ & 0,092 & 0,104 & 0,008 & 96,46 & 0,006 & 0,047 & 0,058 & 0,002 & 97,77 & 0,006 & 0,018 & 0,032 & 0,000 & 98,54 \\
\hline$\phi_{1}$ & 0,031 & 0,101 & 0,117 & 0,011 & 96,54 & 0,010 & 0,072 & 0,089 & 0,005 & 97,77 & $-0,018$ & 0,020 & 0,035 & 0,001 & 98,15 \\
\hline$\phi_{2}$ & $-0,038$ & 0,090 & 0,109 & 0,010 & 96,15 & $-0,029$ & 0,086 & 0,103 & 0,008 & 97,23 & $-0,008$ & 0,014 & 0,027 & 0,000 & 98,54 \\
\hline & \multicolumn{5}{|c|}{$\widetilde{\tau}=0,25$} & \multicolumn{5}{|c|}{$\widetilde{\tau}=0,30$} & \multicolumn{5}{|c|}{$\widetilde{\tau}=0,35$} \\
\hline$\beta_{1}$ & 0,062 & 0,032 & 0,043 & 0,005 & 78,77 & 0,080 & 0,093 & 0,109 & 0,015 & 92,31 & 0,097 & 0,106 & 0,116 & 0,021 & 89,62 \\
\hline$\beta_{2}$ & 0,001 & 0,050 & 0,063 & 0,003 & 97,38 & 0,001 & 0,090 & 0,106 & 0,008 & 97,15 & 0,013 & 0,091 & 0,104 & 0,008 & 96,77 \\
\hline$\phi_{1}$ & 0,021 & 0,088 & 0,105 & 0,008 & 96,92 & 0,044 & 0,091 & 0,102 & 0,010 & 94,38 & $-0,025$ & 0,104 & 0,117 & 0,011 & 96,31 \\
\hline$\phi_{2}$ & $-0,018$ & 0,042 & 0,058 & 0,002 & 97,85 & $-0,048$ & 0,088 & 0,100 & 0,010 & 94,54 & $-0,044$ & 0,094 & 0,111 & 0,011 & 96,23 \\
\hline & \multicolumn{5}{|c|}{$\overline{\bar{\tau}}=0,40$} & \multicolumn{5}{|c|}{$\bar{\tau} \tilde{\tau}=0,45$} & \multicolumn{5}{|c|}{$\overline{\bar{\tau}}=0,50$} \\
\hline$\beta_{1}$ & 0,143 & 0,099 & 0,117 & 0,030 & 83,92 & $-0,135$ & 0,107 & 0,123 & 0,030 & 98,54 & 0,216 & 0,133 & 0,143 & 0,064 & 71,00 \\
\hline$\beta_{2}$ & 0,011 & 0,098 & 0,113 & 0,010 & 95,54 & 0,014 & 0,111 & 0,129 & 0,013 & 96,69 & 0,008 & 0,122 & 0,140 & 0,015 & 96,38 \\
\hline$\phi_{1}$ & $-0,027$ & 0,106 & 0,125 & 0,012 & 96,23 & 0,047 & 0,114 & 0,129 & 0,015 & 95,46 & 0,005 & 0,131 & 0,145 & 0,017 & 96,00 \\
\hline$\phi_{2}$ & $-0,036$ & 0,122 & 0,132 & 0,016 & 94,85 & 0,008 & 0,125 & 0,142 & 0,016 & 96,62 & 0,006 & 0,134 & 0,151 & 0,018 & 96,15 \\
\hline
\end{tabular}


Tabela C.16: Estimativas das Réplicas TSAQV $n=300$ e $n=1000$ - M-spline (Frank)

\begin{tabular}{|c|c|c|c|c|c|c|c|c|c|c|c|c|c|c|c|}
\hline \multicolumn{16}{|c|}{ Cenário: $n=300, N=1300, \alpha_{t}=2, \lambda_{t}=2, \tau=0,20$ e $\alpha_{c}=\frac{1}{5}$} \\
\hline & \multicolumn{5}{|c|}{$\widetilde{\tau}=0,10$} & \multicolumn{5}{|c|}{$\widetilde{\tau}=0,15$} & \multicolumn{5}{|c|}{$\widetilde{\tau}=0,20$} \\
\hline & Viés & EPMC & EPAM & EQM & $\mathrm{CP} \%$ & Viés & EPMC & EPAM & EQM & $\mathrm{CP} \%$ & Viés & EPMC & EPAM & EQM & $\mathrm{CP} \%$ \\
\hline$\beta_{1}$ & 0,029 & 0,129 & 0,148 & 0,017 & 96,77 & 0,016 & 0,095 & 0,109 & 0,009 & 97,23 & $-0,018$ & 0,062 & 0,080 & 0,004 & 98,31 \\
\hline$\beta_{2}$ & $-0,003$ & 0,095 & 0,107 & 0,009 & 96,38 & 0,008 & 0,077 & 0,092 & 0,006 & 97,15 & 0,006 & 0,032 & 0,050 & 0,001 & 98,31 \\
\hline$\phi_{1}$ & 0,033 & 0,137 & 0,150 & 0,020 & 95,92 & 0,012 & 0,099 & 0,114 & 0,010 & 96,69 & $-0,018$ & 0,066 & 0,079 & 0,005 & 96,46 \\
\hline \multirow[t]{2}{*}{$\phi_{2}$} & $-0,037$ & 0,090 & 0,108 & 0,009 & 96,77 & $-0,027$ & 0,089 & 0,099 & 0,009 & 95,77 & $-0,008$ & 0,047 & 0,066 & 0,002 & 98,15 \\
\hline & \multicolumn{5}{|c|}{$\widetilde{\tau}=0,25$} & \multicolumn{5}{|c|}{$\widetilde{\tau}=0,30$} & \multicolumn{5}{|c|}{$\widetilde{\tau}=0,35$} \\
\hline$\beta_{1}$ & 0,057 & 0,109 & 0,121 & 0,015 & 94,62 & 0,081 & 0,124 & 0,141 & 0,022 & 93,69 & 0,095 & 0,128 & 0,145 & 0,025 & 93,15 \\
\hline$\beta_{2}$ & $-0,003$ & 0,088 & 0,103 & 0,008 & 97,31 & 0,003 & 0,101 & 0,112 & 0,010 & 97,00 & 0,010 & 0,105 & 0,117 & 0,011 & 96,31 \\
\hline$\phi_{1}$ & 0,017 & 0,087 & 0,099 & 0,008 & 96,69 & 0,046 & 0,092 & 0,105 & 0,011 & 94,85 & $-0,028$ & 0,104 & 0,117 & 0,012 & 96,23 \\
\hline$\phi_{2}$ & $-0,021$ & 0,080 & 0,099 & 0,007 & 97,77 & $-0,046$ & 0,099 & 0,117 & 0,012 & 96,23 & $-0,047$ & 0,107 & 0,124 & 0,014 & 96,00 \\
\hline & \multicolumn{5}{|c|}{$\widetilde{\tau}=0,40$} & \multicolumn{5}{|c|}{$\widetilde{\tau}=0,45$} & \multicolumn{5}{|c|}{$\widetilde{\tau}=0,50$} \\
\hline$\beta_{1}$ & 0,143 & 0,133 & 0,149 & 0,038 & 88,77 & $-0,146$ & 0,141 & 0,157 & 0,041 & 98,54 & 0,223 & 0,153 & 0,165 & 0,073 & 75,15 \\
\hline$\beta_{2}$ & 0,011 & 0,112 & 0,129 & 0,013 & 96,00 & 0,004 & 0,121 & 0,140 & 0,015 & 97,15 & 0,015 & 0,133 & 0,144 & 0,018 & 95,92 \\
\hline$\phi_{1}$ & $-0,027$ & 0,110 & 0,120 & 0,013 & 94,92 & 0,037 & 0,121 & 0,133 & 0,016 & 95,77 & 0,012 & 0,131 & 0,144 & 0,017 & 96,15 \\
\hline \multirow[t]{4}{*}{$\phi_{2}$} & $-0,037$ & 0,111 & 0,123 & 0,014 & 94,85 & $-0,003$ & 0,122 & 0,138 & 0,015 & 96,77 & 0,013 & 0,132 & 0,142 & 0,018 & 95,77 \\
\hline & \multicolumn{14}{|c|}{ Cenário: $n=1000, N=1300, \alpha_{t}=2, \lambda_{t}=2, \tau=0,20$ e $\alpha_{c}=\frac{1}{5}$} & \\
\hline & Viés & EPMC & EPAM & EQM & $\mathrm{CP} \%$ & Viés & EPMC & EPAM & EQM & $\mathrm{CP} \%$ & Viés & EPMC & EPAM & EQM & $\mathrm{CP} \%$ \\
\hline & \multicolumn{5}{|c|}{$\widetilde{\tau}=0,10$} & \multicolumn{5}{|c|}{$\widetilde{\tau}=0,15$} & \multicolumn{5}{|c|}{$\widetilde{\tau}=0,20$} \\
\hline$\beta_{1}$ & 0,003 & 0,075 & 0,088 & 0,006 & 97,69 & 0,013 & 0,056 & 0,069 & 0,003 & 97,46 & $-0,028$ & 0,021 & 0,038 & 0,001 & 98,54 \\
\hline$\beta_{2}$ & $-0,003$ & 0,086 & 0,102 & 0,007 & 97,08 & 0,007 & 0,071 & 0,088 & 0,005 & 97,77 & 0,006 & 0,012 & 0,023 & 0,000 & 98,46 \\
\hline$\phi_{1}$ & 0,032 & 0,094 & 0,112 & 0,010 & 96,54 & 0,012 & 0,062 & 0,072 & 0,004 & 97,23 & $-0,058$ & 0,025 & 0,036 & 0,004 & 73,85 \\
\hline$\phi_{2}$ & $-0,037$ & 0,049 & 0,067 & 0,004 & 96,69 & $-0,026$ & 0,090 & 0,105 & 0,009 & 96,54 & $-0,013$ & 0,011 & 0,021 & 0,000 & 98,31 \\
\hline & \multicolumn{5}{|c|}{$\widetilde{\tau}=0,25$} & \multicolumn{5}{|c|}{$\widetilde{\tau}=0,30$} & \multicolumn{5}{|c|}{$\widetilde{\tau}=0,35$} \\
\hline$\beta_{1}$ & 0,062 & 0,032 & 0,042 & 0,005 & 76,46 & 0,079 & 0,098 & 0,109 & 0,016 & 91,54 & 0,131 & 0,097 & 0,109 & 0,027 & 81,23 \\
\hline$\beta_{2}$ & 0,000 & 0,051 & 0,070 & 0,003 & 98,08 & 0,002 & 0,095 & 0,107 & 0,009 & 96,46 & 0,005 & 0,096 & 0,115 & 0,009 & 97,31 \\
\hline$\phi_{1}$ & 0,020 & 0,090 & 0,104 & 0,008 & 96,54 & 0,043 & 0,096 & 0,106 & 0,011 & 94,31 & $-0,031$ & 0,098 & 0,108 & 0,011 & 95,31 \\
\hline$\phi_{2}$ & $-0,028$ & 0,042 & 0,052 & 0,003 & 95,00 & $-0,052$ & 0,093 & 0,110 & 0,011 & 95,23 & $-0,047$ & 0,099 & 0,111 & 0,012 & 94,85 \\
\hline & \multicolumn{5}{|c|}{$\overline{\bar{\tau}}=0,40$} & \multicolumn{5}{|c|}{$\overline{\bar{\tau}}=0,45$} & \multicolumn{5}{|c|}{$\overline{\bar{\tau}}=0,50$} \\
\hline$\beta_{1}$ & 0,149 & 0,101 & 0,112 & 0,032 & 79,23 & $-0,109$ & 0,105 & 0,122 & 0,023 & 98,54 & 0,089 & 0,124 & 0,135 & 0,023 & 92,31 \\
\hline$\beta_{2}$ & 0,005 & 0,100 & 0,114 & 0,010 & 95,77 & 0,010 & 0,106 & 0,116 & 0,011 & 96,23 & 0,010 & 0,113 & 0,129 & 0,013 & 96,92 \\
\hline$\phi_{1}$ & $-0,031$ & 0,108 & 0,119 & 0,013 & 95,08 & 0,043 & 0,116 & 0,135 & 0,015 & 96,23 & 0,007 & 0,132 & 0,148 & 0,017 & 96,38 \\
\hline$\phi_{2}$ & $-0,040$ & 0,105 & 0,124 & 0,013 & 95,85 & 0,008 & 0,117 & 0,133 & 0,014 & 96,69 & 0,009 & 0,127 & 0,141 & 0,016 & 96,23 \\
\hline
\end{tabular}




\section{Referências Bibliográficas}

Akaike, H. (1974). A new look at the statistical model identification, Selected Papers of Hirotugu Akaike, Springer, pp. 215-222.

Bertsekas, D. P. (2014). Constrained optimization and Lagrange multiplier methods, Academic press.

Bolbolian, G. M., Eghbal, N., Amini, M., Azarnoosh, H. A. e Bozorgnia, A. (2010). Aspects of dependence in cuadras-auge family, Communications in Statistics-Theory and Methods 39(11): 2094-2107.

Campolina, A. G. e Ciconelli, R. M. (2006). Qualidade de vida e medidas de utilidade: parâmetros clínicos para as tomadas de decisão em saúde, Revista Panamericana de Salud Pública 19: 128-136.

Cesar, R. C. C. (2013). Estimação e comparação de curvas de sobrevivência sob censura informativa., PhD thesis, Universidade de São Paulo.

Chandra, K. (2015). Survival Analysis using Bivariate Archimedean Copulas, PhD thesis, Columbia University.

Clayton, D. G. (1978). A model for association in bivariate life tables and its application in epidemiological studies of familial tendency in chronic disease incidence, Biometrika 65: $141-152$.

Colosimo, E. A. e Giolo, S. R. (2006). Análise de sobrevivência, São Paulo: Edgard Blücher .

Cox, D., Fitzpatrick, R., Fletcher, A., Gore, S., Spiegelhalter, D. e Jones, D. (1992). Quality-of-life assessment: can we keep it simple?, Journal of the Royal Statistical Society: Series A (Statistics in Society) 155(3): 353-375.

Cox, D. R. (1972). Regression models and life-tables, Journal of the Royal Statistical Society: Series B (Methodological) 34(2): 187-202.

Cuadras, C. M. e Augé, J. (1981). A continuous general multivariate distribution and its properties, Communications in Statistics-Theory and Methods 10(4): 339-353.

Efron, B. (1967). The two sample problem with censored data, Proceedings of the fifth Berkeley symposium on mathematical statistics and probability, Vol. 4, pp. 831-853.

Embrechts, P., Lindskog, F. e McNeil, A. (2001). Modelling dependence with copulas, Rapport technique, Département de mathématiques, Institut Fédéral de Technologie de Zurich, Zurich. 
Emura, T. e Chen, Y.-H. (2016). Gene selection for survival data under dependent censoring: a copula-based approach, Statistical methods in medical research 25(6): 2840-2857.

Emura, T. e Chen, Y.-H. (2018). Analysis of Survival Data with Dependent Censoring: Copula-based Approaches, Springer.

Ferreira, L. d. N. (2002). Utilidades, qalys e medição da qualidade de vida [utilities, qalys and quality of life measurement] documento de trabalho n. ${ }^{\circ}$ 1/2002, Associação Portuguesa de Economia da Saúde.

Fine, J. P. e Gelber, R. D. (2001). Joint regression analysis of survival and quality-adjusted survival, Biometrics 57(2): 376-382.

Fine, J. P., Jiang, H. e Chappell, R. (2001). On semi-competing risks data, Biometrika 88(4): 907919.

Fleck, M., Lousada, S., Xavier, M., Chachamovich, E., Vieira, G., Santos, L. e Pinzon, V. (1999). Application of the portuguese version of the instrument for the assessment of the quality of life of the world health organization (whoqol-100), Revista de saude publica 33(2): 198-205.

Frank, M. J. (1979). On the simultaneous associativity off $(\mathrm{x}, \mathrm{y})$ and $\mathrm{x}+\mathrm{y}-\mathrm{f}(\mathrm{x}, \mathrm{y})$, Aequationes mathematicae 19(1): 194-226.

Friedman, J., Hastie, T. e Tibshirani, R. (2001). The elements of statistical learning, Vol. 1, Springer series in statistics New York.

Gelber, R. D., Gelman, R. S. e Goldhirsch, A. (1989). A quality-of-life-oriented endpoint for comparing therapies, Biometrics 45(3): 781-795.

Gelber, R. D., Goldhirsch, A. e Cavalli, F. (1991). Quality-of-life-adjusted evaluation of adjuvant therapies for operable breast cancer, Annals of internal medicine 114(8): 621-628.

Gelber, R. D., Goldhirsch, A., Cole, B. F., Group, I. B. C. S. et al. (1993). Parametric extrapolation of survival estimates with applications to quality of life evaluation of treatments, Controlled clinical trials 14(6): 485-499.

Genetics Home Reference (2020). Condition summary: Lynch syndrome. National Library of Medicine (US). Bethesda (MD).

URL: https://ghr.nlm.nih.gov/condition/lynch-syndrome\#

Glasziou, P., Simes, R. e Gelber, R. (1990). Quality adjusted survival analysis, Statistics in medicine 9(11): 1259-1276.

Goldhirsch, A., Gelber, R. D., Simes, R. J., Glasziou, P. e Coates, A. S. (1989). Costs and benefits of adjuvant therapy in breast cancer: a quality-adjusted survival analysis., Journal of Clinical Oncology 7(1): 36-44.

Group, W. et al. (1995). The world health organization quality of life assessment (WHOQOL): position paper from the world health organization, Social science $\mathcal{E}$ medicine 41(10): 14031409.

Gumbel, E. J. (1960). Distributions des valeurs extremes en plusiers dimensions, Publ. Inst. Statist. Univ. Paris 9: 171-173. 
Harrell, F. E., Califf, R. M., Pryor, D. B., Lee, K. L. e Rosati, R. A. (1982). Evaluating the yield of medical tests, Jama 247(18): 2543-2546.

Hougaard, P. (1986). A class of multivanate failure time distributions, Biometrika 73(3): 671-678.

Huang, X. e Zhang, N. (2008). Regression survival analysis with an assumed copula for dependent censoring: a sensitivity analysis approach, Biometrics 64(4): 1090-1099.

Hutchinson, T. e Lai, C. (1990). Continuous bivariate distributions, Emphasising Applications, Rumsby, Adelaide.

James, G., Witten, D., Hastie, T. e Tibshirani, R. (2013). An introduction to statistical learning, Vol. 112 , Springer.

Kalbfleisch, J. D. e Prentice, R. L. (2002). The statistical analysis of failure time data, Vol. 360, John Wiley \& Sons.

Kaplan, E. L. e Meier, P. (1958). Nonparametric estimation from incomplete observations, Journal of the American statistical association 53(282): 457-481.

Lagakos, S. W. (1979). General right censoring and its impact on the analysis of survival data, Biometrics pp. 139-156.

Lakhal-Chaieb, L., Jenkins, M., Buchanan, D., Hopper, J., Win, A. K., Briollais, L. e Choi, Y.-H. (2017). Modeling of successive cancer risks in lynch syndrome families in the presence of competing risks using copulas, Biometrics 73(1): 271-282.

Luenberger, D. G. (1997). Optimization by vector space methods, John Wiley \& Sons.

Ma, J. (2010). Positively constrained multiplicative iterative algorithm for maximum penalized likelihood tomographic reconstruction, IEEE Transactions on Nuclear Science 57(1): 181-192.

Ma, J., Heritier, S. e Lô, S. N. (2014). On the maximum penalized likelihood approach for proportional hazard models with right censored survival data, Computational Statistics $\mathcal{E}$ Data Analysis 74: 142-156.

Mantel, N. (1966). Evaluation of survival data and two new rank order statistics arising in its consideration, Cancer Chemother. Rep. 50: 163-170.

Mari, D. D. e Kotz, S. (2001). Correlation and dependence, World Scientific.

Marotti, M. (2007). Quais são os objetivos clínicos que determinam a eficácia dos tratamentos em oncologia?, Revista da Associação Médica Brasileira 53(6): 477-478.

Nelsen, R. B. (2006). An Introduction to Copulas, 2nd edn, New York: Springer Series in Statistics, Springer-Verlag.

Normilio-Silva, K. (2015). Qualidade de vida relacionada à saúde e tempo de sobrevida ajustado para a qualidade de vida em pacientes com câncer internados em unidades de terapia intensiva, $\mathrm{PhD}$ thesis, Universidade de São Paulo.

Normilio-Silva, K., de Figueiredo, A. C. et al. (2016). Long-term survival, quality of life, and quality-adjusted survival in critically ill patients with cancer, Critical care medicine 44(7): 1327-1337.

Ospina, R. e Ferrari, S. L. (2010). Inflated beta distributions, Statistical Papers 51(1): 111. 
O'Sullivan, F. (1988). Fast computation of fully automated log-density and log-hazard estimators, SIAM Journal on scientific and statistical computing 9(2): 363-379.

Peto, R. e Peto, J. (1972). Asymptotically efficient rank invariant test procedures, Journal of the Royal Statistical Society: Series A (General) 135(2): 185-198.

Prieto, L. e Sacristán, J. A. (2003). Problems and solutions in calculating quality-adjusted life years (qalys), Health and quality of life outcomes 1(1): 80.

Ramsay, J. O. et al. (1988). Monotone regression splines in action, Statistical science 3(4): 425-441.

Rivest, L.-P. e Wells, M. T. (2001). A martingale approach to the copula-graphic estimator for the survival function under dependent censoring, Journal of Multivariate Analysis 79(1): 138155.

Robert, C. P., Casella, G. e Casella, G. (2010). Introducing monte carlo methods with r, Vol. 18, Springer.

Rotnitzky, A., Bergesio, A. e Farall, A. (2009). Analysis of quality-of-life adjusted failure time data in the presence of competing, possibly informative, censoring mechanisms, Lifetime data analysis 15(1): 1-23.

Roussas, G. G. (1997). A course in Mathematical Statistics, 2nd edn, Elsevier.

Schweizer, B. e Wolff, E. F. (1981). On nonparametric measures of dependence for random variables, The Annals of Statistics 9: 879-885.

Seidl, E. M. F. e Zannon, C. M. L. d. C. (2004). Qualidade de vida e saúde: aspectos conceituais e metodológicos, Cadernos de saúde pública 20: 580-588.

Shen, B.-J., Myers, H. F. e McCreary, C. P. (2006). Psychosocial predictors of cardiac rehabilitation quality-of-life outcomes, Journal of psychosomatic research 60(1): 3-11.

Shih, J.-H. e Emura, T. (2018). Likelihood-based inference for bivariate latent failure time models with competing risks under the generalized fgm copula, Computational Statistics 33(3): 1293-1323.

Sklar, A. (1959). Fonctions de répartition à $n$ dimensions et leurs marges, Annales de l'ISUP 8(229-231): 229-231.

Tsiatis, A. (1975). A nonidentifiability aspect of the problem of competing risks, Proceedings of the National Academy of Sciences 72(1): 20-22.

Tunes-da Silva, G., Sen, P. K. e Pedroso-de Lima, A. C. (2008). Estimation of the mean qualityadjusted survival using a multistate model for the sojourn times, Journal of Statistical Planning and Inference 138(8): 2267-2282.

Van Der Laan, M. J. e Hubbard, A. (1999). Locally efficient estimation of the quality-adjusted lifetime distribution with right-censored data and covariates, Biometrics 55(2): 530-536.

Wang, A. (2012). On the nonidentifiability property of archimedean copula models under dependent censoring, Statistics \& Probability Letters 82(3): 621-625.

Wang, A. (2014). Properties of the marginal survival functions for dependent censored data under an assumed archimedean copula, Journal of Multivariate Analysis 129: 57-68. 
Wang, R., Wu, C., Zhao, Y., Yan, X., Ma, X., Wu, M., Liu, W., Gu, Z., Zhao, J. e He, J. (2008). Health related quality of life measured by sf-36: a population-based study in shanghai, china, BMC public health 8(1): 292.

Wienke, A. (2010). Frailty models in survival analysis, Chapman and Hall/CRC.

Williams, J. e Lagakos, S. (1977). Models for censored survival analysis: Constant-sum and variable-sum models, Biometrika 64(2): 215-224.

Xu, J., Ma, J., Connors, M. H. e Brodaty, H. (2018). Proportional hazard model estimation under dependent censoring using copulas and penalized likelihood, Statistics in medicine 37(14): 2238-2251.

Xu, J., Ma, J. e Prvan, T. (2017). Non parametric hazard estimation with dependent censoring using penalized likelihood and an assumed copula, Communications in Statistics-Theory and Methods 46(22): 11383-11403.

Zhan, L. (1992). Quality of life: conceptual and measurement issues, Journal of Advanced Nursing 17(7): 795-800.

Zhao, H. e Tsiatis, A. A. (1997). A consistent estimator for the distribution of quality adjusted survival time, Biometrika 84(2): 339-348.

Zhao, H. e Tsiatis, A. A. (1999). Efficient estimation of the distribution of quality-adjusted survival time, Biometrics 55(4): 1101-1107.

Zhao, H. e Tsiatis, A. A. (2000). Estimating mean quality adjusted lifetime with censored data, Sankhyā: The Indian Journal of Statistics, Series B pp. 175-188.

Zhao, H. e Tsiatis, A. A. (2001). Testing equality of survival functions of quality-adjusted lifetime, Biometrics 57(3): 861-867.

Zheng, M. (1992). On the use of copulas in dependent competing risk theory, PhD thesis, The Ohio State University.

Zheng, M. e Klein, J. P. (1994). A self-consistent estimator of marginal survival functions based on dependent competing risk data and an assumed copula, Communications in StatisticsTheory and Methods 23(8): 2299-2311.

Zheng, M. e Klein, J. P. (1995). Estimates of marginal survival for dependent competing risks based on an assumed copula, Biometrika 82(1): 127-138. 